\title{
Vibration-Rotation-Tunneling Dynamics in Small Water Clusters
}

\author{
Nick Pugliano \\ Ph.D. Thesis \\ Department of Chemistry \\ University of California \\ and \\ Chemical Sciences Division \\ Lawrence Berkeley Laboratory \\ University of California \\ Berkeley, CA 94720
}

November 1992

This work was supported by the Director, Office of Energy Research, Office of Basic Energy Sciences, Chemical Sciences Division of the U.S. Department of Energy under contract No. DE-AC03-76SF00098.

\section{MASTER}




\title{
Vibration-Rotation-Tunneling Dynamics in Small Water Clusters
}

\author{
Copyright $\odot 1992$
}

by

Nick Pugliano

The U.S. Department of Energy has the right to use this thesis for any purpose whatsoever including the right to reproduce all or any part thereof 
Abstract

Vibration-Rotation-Tunneling Dynamics in Small Water Clusters

by

Nick Pugliano

Doctor of Philosophy in Chemistry

University of California at Berkeley

Professor Richard J. Saykally, Chair

The goal of this work is to characterize the intermolecular vibrations of small water clusters. Using tunable far infrared laser absorption spectroscopy, large amplitude vibration-rotation-tunneling (VRT) dynamics in vibrationally excited states of the water dimer and the water trimer are investigated.

This study begins with the measurement of 12 VRT subbands, consisting of approximately 230 transitions, which are assigned to an $82.6 \mathrm{~cm}^{-1}$ intermolecular vibration of the water dimer-d4. Each of the VRT subbands originate from $\mathrm{K}_{\mathrm{a}}{ }^{\prime \prime}=0$ and terminate in either $\mathrm{K}_{\mathrm{a}}{ }^{\prime}=0$ or 1 . These data provide a complete characterization of the tunneling dynamics in the vibrationally excited state as well as definitive symmetry labels for all VRT energy levels. Furthermore, an accurate value for the A' rotational constant, of 122.9 $\mathrm{GHz}$, is found to agree well with its corresponding ground state value. All other excited state rotational constants are fitted, and discussed in terms of the corresponding ground state constants. In this vibration, the quantum tunneling motions are determined to exhibit large dependencies with both the $\mathrm{K}_{\mathrm{a}}$ ' quantum number and the vibrational coordinate, as is evidenced by the measured tunneling splittings. The generalized internal-axis-method (IAM) treatment, which has been developed to model the tunneling dynamics, is considered for the qualitative description of each tunneling pathway, however, the variation of tunneling splittings with vibrational excitation indicate that the high barrier approximation 
does not appear to be applicable for this vibrational coordinate. The data are consistent with a motion possessing a' symmetry, and the vibration is assigned as the $v_{12}$ acceptor bending coordinate. This assignment is in agreement with the vibrational symmetry, the results of high level ab initio calculations, and preliminary data assigned to the analogous vibration in the $\mathrm{D}_{2} \mathrm{O}$ - $\mathrm{DOH}$ isotopomer.

Also, in an effort to begin the study of larger water clusters, so that the influence of non-pairwise additive forces and concerted tunneling dynamics of many-body systems may be examined, the first detailed experimental study of the cyclic water trimer is reported. A vibrational band of the perdeuterated cluster has been measured near $89.4 \mathrm{~cm}^{-1}$, using tunable far infrared lasers, and a group theoretical model is formulated to explain the observed VRT structure. From this model, the measured tunneling splittings establish that

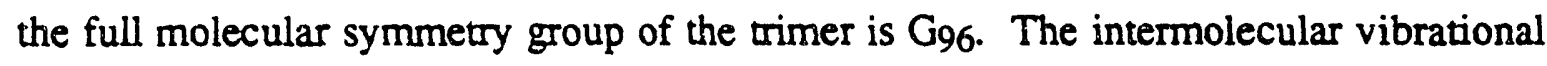
motion is assigned as a coordinate which involves a librational motion of the water subunits about the hydrogen bond axis. This assignment is supported by the group theory and high level $a b$ initio calculations. The data indicate a chiral six-membered ring structure with rapid ( $>10 \mathrm{ps)}$ quantum tunneling processes interconverting the enantiomers. It is speculated that larger water clusters will also exhibit a transient chirality, and that similar effects may be found in liquid water. 
This dissertation is lovingly dedicated

to

Sandra Jo 
Table of Contents

Chapter 1 An Introduction to the Study of Water .................... 1

1.1 Introduction $\quad$.................................................... 1

1.2 Theoretical Intermolecular Pair Potentials .................... 2

1.3 The Molecular Dynamics of Liquid Water ................... 6

1.4 Conclusion $\quad$ C................................................. 9

Chapter 2 The Far Infrared Laser Spectrometer ....................... 14

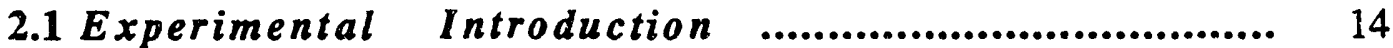

2.2 The Apollo $\mathrm{CO}_{2}$ Laser ..................................... 16

2.3 The Far Infrared Laser ...................................... 21

2.4 The Schottky Barrier Diode .................................... 28

2.5 Tunable Microwaves $\quad$.................................... 33

2.5.1 General Microwave Technology; 2 - $20 \mathrm{GHz}$................. 33

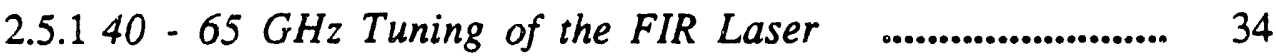

2.5.2 $65-110 \mathrm{GHz}$ Tuning of the FIR Laser ........................ 38

2.6 Detectors; Ge:Ga Photoconductors ........................ 42

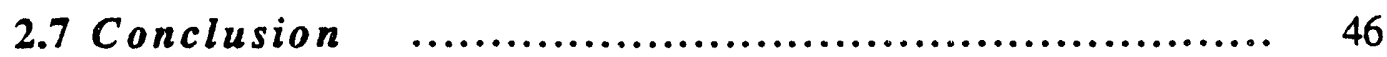

Chapter 3 Vibrational and $K_{a}{ }^{\prime}$ Dependencies of the

Multidimensional Tunneling Dynamics in the $82.6 \mathrm{~cm}^{-1}$

Intermolecular Vibration of the Water Dimer-d $d_{4} \ldots . . . . . . . . . . . . . . . . . . . . . \quad 50$

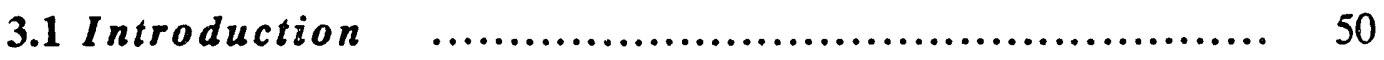


3.2 Water Dimer Experimental Conditions ...................... 52

3.3 Results and Analysis ..................................... 53

3.3.1 Group Theoretical Considerations ............................ 53

3.3.2 Spectroscopic Implications and Notation Definitions ............ 60

3.3.3 $K_{a}=0 \rightarrow 0$ VRT Subbands ................................ 68

3.3.4 $K_{a}=0 \quad->1$ VRT Subbands ................................ 76

3.4 The Tunneling Splittings ................................ 83

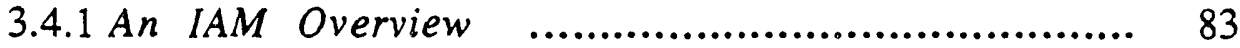

3.4.2 Acceptor Tunneling ...................................... 84

3.4.3 Donor-Acceptor Interchange Tunneling ........................ 88

3.4.4 Donor Tunneling ........................................... 90

3.4.5 $\mathrm{K}_{a}$ Dependence of Tunneling ............................. 91

3.5 The Intermolecular Vibrational Coordinate ................... 93

3.5.1 The Vibrational Assignment ................................ 93

3.5.2 Isotopically Substituted VRT Bands of the Water Dimer ........ 96

3.5.3 Summary of VRT Analysis .............................. 99

3.5.4 Basis Functions ........................................... 101

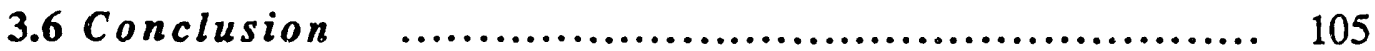

Chapter 4 The Measurement of Vibration-Rotation-Tunneling

Dynamics in the Water Trimer-d 6 Cluster .......................... 109

4.1 Introaluction $\quad$................................................. 109

4.2 Experimental/Isotope Experimental Verification ............ 112

4.3 Results $\quad$................................................... 113

4.4 VRT Multiplet Interpretation $\quad$............................... 120

4.4.1 The Formulation of the Full Molecular Symmetry Group ....... 120 
4.4.2 Interpretation of the Observed Multiplet Structure and the

Vibrational Assignment ............................... 138

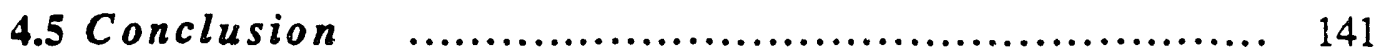

Chapter 5 Concluding Remarks.................................. 147

5.1 Future Work $\quad$ W....................................... 147 


\section{Acknowledgements}

Although these pages attempt to acknowledge all of the people who have influenced my graduate studies at Berkeley, it is almost impossible to do so, because the list is so incredibly long. Rich Saykally is certainly on the top of this list, and it is most difficult to adequately express the role he has played in the successful completion of this doctoral degree. I thank him for presenting me with exciting research possibilities at a time when my scientific goals did not seem realistic. He is an individual who strives for excellence through his creativity, intelligence, and constant drive, and I am pleased that I had the chance to work with such a well rounded person.

There are also many brilliant members of the Saykally group who have taught me a great deal about science and life. Ron Cohen (who kept me on my toes in lab and throughout the city of Berkeley), was the person who first attracted me to the FIR project, and I will always br. grateful for this. Special thanks also go out to Charlie Schmuttenmaer and Kerry Busarow who helped introduce me to FIR lasers and the methods of data analysis, whereas Jenny Loeser and Matt Elrod have most recently contributed to my understanding of complex intermolecular dynamics. I've learned a great deal from each of these friends, and I thank them all. My experiences with other members of the FIR group have also been extremely valuable. Jeff Cruzan, Bryan Host, and Kun Liu have been excellent additions to the FIR effort, and will certainly be remembered as they continue to push the limits of the spectrometer. The other members of the group, both past and present, have provided me with constant support. Their efforts toward experimentation and good times will certainly be missed. In particular Jim Heath, Eric Keim, Luca Dore, Dave Steyert, Dave Cook, Jeff Rzepiela, Jim Scherer, Stephan Schlemmer, Alan Van Orden, Erica Kuo, Ray Fellers, Pat Collier, and Josh Paul have each contributed to my education in an important way, and I thank all of them. 
My interaction with many other groups has been a positive experience throughout my six years at Berkeley. In the early days, the groups of Herb Strauss, Brad Moore, and Y. T. Lee were particularly generous in lending me many pieces of equipment to try those, "one shot" experiments. (I hope I've returned everything.) Also, my "organic" friends, Bob Hendricks, Mike Malaska, Alan Kaplan, and Felicia Etzkorn forced my science to maintain a level of diversity which is appreciated.

Most recently, the groups of Eugene Haller in the Material Science Department at Berkeley and Tom Crowe at the University of Virginia, were critical in the successful exploration of technological advances related to the FIR spectrometer. Finally, my relationship with Geoff Blake has always been an instructive one, and I particularly thank him for his time and patience over the last two years. I only hope that my FIR colleagues will continuously maintain contact with these groups in the furure.

Before joining the FIR project, I had the privilege of working with the research group of George Pimentel. My experiences with George will never be forgotten, for he allowed me to explore many scientific problems ranging from "photon catalysis" to "mode selectivity", as well as many others. In those days, many of these experiments did not produce the desired result. But in times of trouble George v'ould always provide me with a glimmer of hope. A. with Rich, nothing was impossible, and I value this visionary Berkeley approach to science. Although the work conducted with George is not represented in this manuscript, it would be an injustice to those important early days if credit were not given to the other people who guided me. Mark Young, Brent Wurfel, Vicki Grassian, Heinz Frei, Jim Harrison, Ed Orton, and Merideth Morgan provided valuable insight into my many early scientific endeavors as well as many memories.

It is imperative that I thank my entire family, especially my father and mother, Victor and Mary Jane, for without their support this dissertation would have never been possible. Finally, my wife, Sandra Jo has been the most influential person in my life. She 
has helped me in numerous ways throughout my graduate work, and my thanks go out to her.

In closing, I would like to thank the generous support provided by the Director, Office of Energy Research, Office of Basic Energy Sciences, Chemical Sciences Division of the U. S. Department of Energy under contract No. DE-AC03-76SF00098. 


\section{Chapter 1}

\section{An Introduction to the Study of Water}

\subsection{Introduction}

Due to its obvious importance in chemistry and biology, an incredibly large effort has surrounded the investigation of the properties of water in both its gaseous and liquid phase. A review of relevant theory and experiment is best summarized in the seven volume treatise of Franks, ${ }^{1}$ and the text by Eisenberg and Kauzmann. ${ }^{2}$ Although a great deal of work has been acomplished, there still remain important unanswered questions which have potentially significant impact on many chemical and biological problems. In particular, the details of solvation processes, ${ }^{3}$ phase changes, ${ }^{4}$ and the correct description of the intermolecular forces which exist between water molecules 5 have been current topics of interest. Recently, a great deal of effort has also been directed toward understanding the role of tunneling and librational motions with regard to many aqueous phase processes. These considerations are critical elements in gaining the necessary insight related to many aqueous phase chemical phenomena, such as electron transfer, and the structure of liquid water itself. ${ }^{6}$ To achieve an adequate description of the forces and dynamics sperating in water, it is imperative that experimental information is gathered for the intermolecular vibrations of small water clusters, because an understanding of these motions is proving to be extremely influential in the full characterization of the aqueous phase. This will be demonstrated in this chapter, for it provides part of the motivation behind the experiments described throughout this dissertation. 
Although it is important to examine water in the form of clusters, very little work has been accomplished to date. Prior to the studies contained within, no intermolecular vibrations have been examined with high resolution spectroscopic probes. Certainly the large body of data relating to the ground state of the water dimer has been of value in terms of its structural and dynamical aspects. However, this characterization is confined to the intermolecular potential energy surface (IPS) minimum, because it samples the $\mathrm{v}=0$ manifold. Furtherrnore, no detailed information has been previously obtained for larger water clusters. The experiments related to clusters of water are reported in the references, 7 and upon inspection of these results it becomes clear that a great deal of important work is necessary.

Two measurements are reported in this dissertation. The first represents the only detailed characterization of an intermolecular vibration for the water dimer. A vibrationrotation-tunneling (VRT) band of the water dimer-d4 has been observed near $82.6 \mathrm{~cm}^{-1}$, and this work is presented in Chapter III. In Chapter IV, the first detailed experimental characterization of the cyclic form of the water trimer is described through the analysis of an intermolecular vibration of the perdeuterated isotopomer near $89.6 \mathrm{~cm}^{-1}$. Using the high spectral resolution of the Berkeley tunable far infrared laser spectrometer, the VRT dynamics in both of these clusters have been characterized for these vibrations. The remainder of this introduction will present a brief discussion of the theoretical efforts involving the liquid phase of water. Particular emphasis will be placed on properties which are though to aid in the understanding of pairwise and nonpairwise additive intermolecular forces, and the tunneling and large amplitude motions in condensed phase water.

\subsection{Theoretical Intermolecular Pair Potentials}

The study of binary clusters permits the details of the pairwise intermolecular forces to be characterized without the complication of many body forces. Until this thesis work no intermolecular vibration of the water dimer had been detected with a high resolution 
spectroscopic probe. However, a large number of theoretical groups have attempted to calculate the IPS of water. Many of these calculations have been used for the prediction of the intermolecular vibrational frequencies for the dimer, 8 to formulate theories which attempt to explain the tunneling dynamics in the ground state, ${ }^{9}$ to calculate structures for larger water clusters, ${ }^{10}$ and to explore a variety of aqueous phase problems. ${ }^{11}$ Although a great deal of effort has been devoted to the IPS, it is difficult to determine the global usefulness of a particular potential because they usually are constructed to address a particular aspect of the water-water interaction.

To illustrate the large range of uncertainty $i_{i}$ the theoretically generated IPS, a list of the predicted harmonic vibrational frequencies for the normal isotopomer of the water dimer is shown in Table 1.1 for several of the more widely used calculations. This table employs the normal mode assignments which are reproduced in Figure 1.1 from Reference 7. A variety of theoretical methods are considered in Table 1.1, and the inspection of the associated references is left up to the interested reader for the details of each. For the purpose of estimating frequency for the intermolecular vibrations, the most reliable calculations are probably those of Frisch et al., 11 or Amos, 12 because for these results the most extensive basis sets have been explored with the highest levels of ab initio theory. The empirical potentials are usually developed to describe a specific property of liquid water (except for the EPEN model), and the RWK2 potential is probably the best attempt at a globally useful IPS. The most definitive way in which an IPS may be tested is to measure the intermolecular vibrations of the water dimer, and to compare the results directly with theory. This thesis work constintes a step in this direction.

Through the study of the water trimer, nonpairwise additive forces arising from a multibody interaction can eventually be addressed. Very few theoretical studies employ nonadditive terms in the interaction energy of a cluster, for it is exceedingly difficult to arrive at a conclusive method for partitioning the energy. Nevertheless, several attempts have been conducted from both an ab initio 5,12 and the molecular dynamics ${ }^{13,14}$ approach. 
From this work it is concluded that the long range induction energy (through induced polarization effects) provides the primary interaction which results in nonadditivity. In

Table 1.1

The predicted intermolecular vibrational frequencies (in $\mathrm{cm}^{-1}$ ) for several theoretical IPS of the water dimer. The last column indicates the method in which the calculation was conducted. The vibrational label is defined according to the normal modes shown in Figure 1.1 .

\begin{tabular}{|c|c|c|c|c|c|c|c|}
\hline & $v_{6}$ & $v_{7}$ & $v_{8}$ & $v_{10}$ & $v_{11}$ & $v_{12}$ & \\
\hline RWK2-M 3 & 483 & 272 & 169 & 782 & 219 & 115 & $\begin{array}{l}\text { Semi-empirical with } \\
\text { Anharmonic Terms }\end{array}$ \\
\hline $\mathrm{CF}^{15}$ & 327 & 120 & 281 & 509 & 307 & 87 & $\begin{array}{l}\text { Central Force } \\
\text { Empirical }\end{array}$ \\
\hline PM616 & 344 & 108 & 338 & 708 & 831 & 132 & $\begin{array}{c}\text { Empirical } \\
\text { Polarization Model }\end{array}$ \\
\hline $\mathrm{BJH}^{17}$ & 333 & 190 & 154 & 537 & 131 & 42 & $\begin{array}{c}\text { Empirical Potential } \\
\text { for Water } \text { Iiq }_{\text {Vibration }} \\
\text { SCF/CI }\end{array}$ \\
\hline MCYL 18 & 350 & 186 & 149 & 620 & 131 & 115 & $\begin{array}{l}\text { Ab Initio } \\
\text { HF }\end{array}$ \\
\hline Frisch et al. ${ }^{19}$ & 377 & 198 & 164 & 661 & 162 & 135 & Ab Initio \\
\hline $\operatorname{Amos}^{20}$ & 356 & 174 & 142 & 589 & 153 & 129 & Ab Initio \\
\hline $\mathrm{MMC}^{21}$ & 352 & 214 & 135 & 547 & 226 & 147 & $\begin{array}{l}\text { Molecular Mechanics } \\
\text { of Clusters/Empirical } \\
\text { SCF }\end{array}$ \\
\hline Swanton et al. ${ }^{22}$ & 322 & 169 & 96 & 541 & 113 & 106 & $\begin{array}{c}\text { Ab Initio } \\
\text { Empirical Potential }\end{array}$ \\
\hline EPEN23 & 496 & 168 & 189 & 593 & 161 & 98 & Electrons and Nuclei \\
\hline
\end{tabular}

Reference 5, a detailed partitioning of the energy is accomplished up to 3rd order in a Moller-Plesset Perturbation Theory treatment for dispersion, polarization, and exchange terms. This study shows that the total three body correction is very small at the minimum energy structure, due to the cancellation from the individual terms. However, it also explores the anisotropy of these terms, and indicates that the three body terms may become more noticeable as the cluster proceeds through low barrier tunneling motions. Thus, the 
Figure 1.1

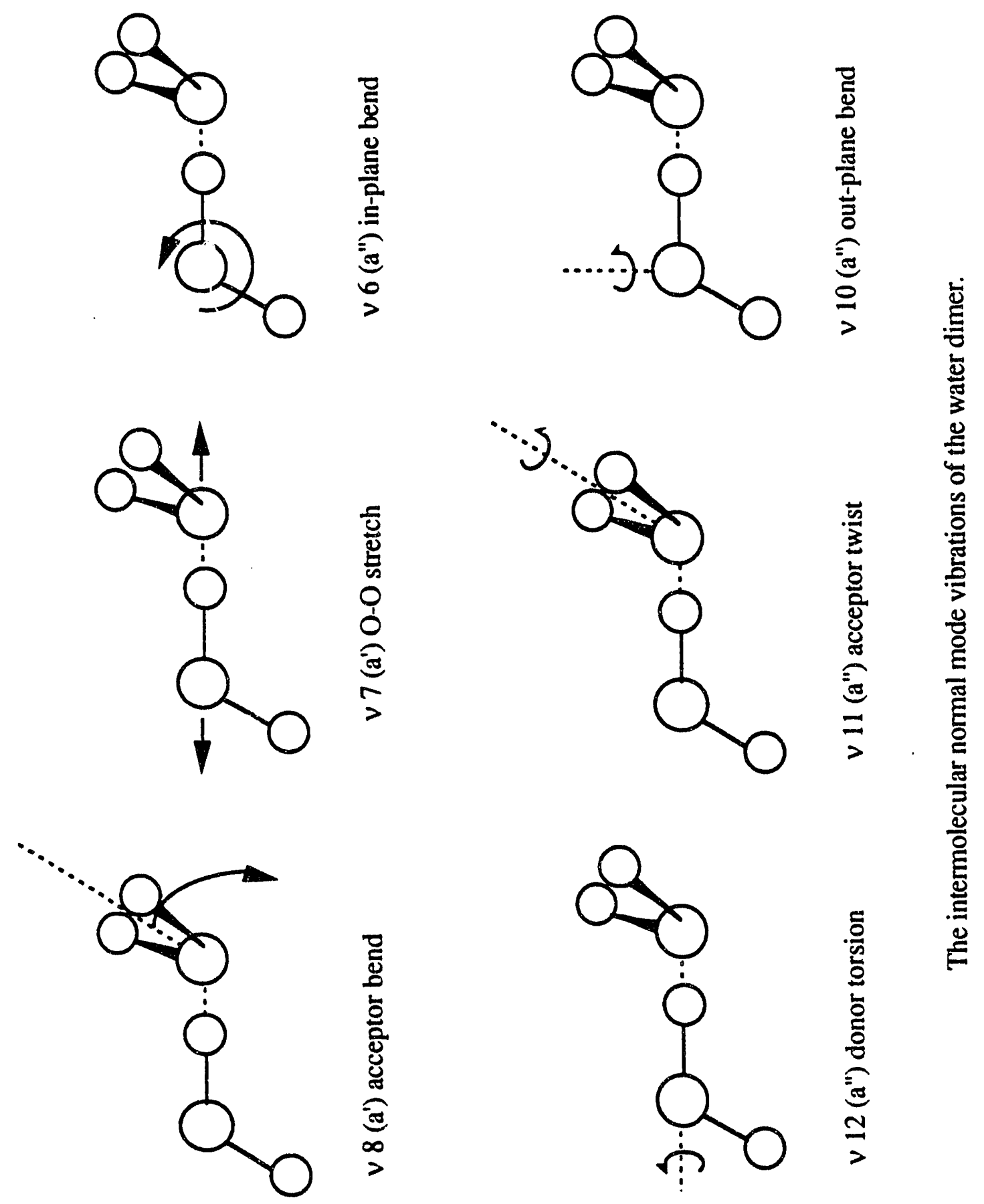


VRT dynamics for the trimer will be valuable in gaining a full appreciation for the nonpairwise additive forces in the water trimer.

\subsection{The Molecular Dynamics of Liquid Water}

The quantum tunneling dynamics in water clusters are characterized by motions which destroy and regenerate the weak hydrogen bond $(\mathrm{H}$-bond) via internal rotations of the substituent water monomers. These motions may be thought of as the cluster counterparts to the reorientational motions present in aqueous phase H-bonded networks, for the timescales upon which the dynamics proceed (10 ps), and possibly even the pathways related to quantum tunneling in the clusters, are similar to motions in liquid water. In particular, tunneling motions in clusters possess an increasing level of significance, because the analogous motions in the liquid phase have been predicted to be responsible for certain anomalous solvent and thermodynamic properties of water. This section will deal with examples in which these properties are addressed.

Modeling of reorientational dynamics in liquid water (and all many body liquids), require the application of sophisticated molecular dynamics (MD) calculations. Unfortunately, to make the simulation tractable, theorists are required to neglect particular aspects of the global problem to address a specific property of interest. However, calculations of this sort provide the most up to date understanding of the aqueous phase problem. With this in mind, a few results from some of the most recent calculations will be presented to illustrate the possible connection from the dynamics in clusters to those of the condensed phase.

Since both tunneling and reorientational processes require the breaking and forming of hydrogen bonds, it is necessary to define what is meant by an H-bond. From a simple perspective, an $\mathrm{H}$-bond is an attractive interaction between a proton donor and a proton acceptor. For the specific case of water, the proton donor and acceptor capabilities are both fully present. H-bonded species are typically characterized by rather distinct chemical 
signatures, such as red shifted $\mathbb{R}$ stretching frequencies and a decrease in nuclear magnetic shielding of several ppm for the proton donating subunit involved. With regard to MD calculations, a more quantitative definition is needed so that a benchmark may be used to compare the reorientational and energetic fluctuations in the liquid. Therefore, within the context of this section, the H-bond will be defined with both an energetic and a geometric constraint. A system will be considered to exhibit $\mathrm{H}$-bonding when the interaction between the constituents is larger than a given energy cutoff which is typically set at approximately 16 kjoules/mole. The second criterion involving geometric considerations define an $\mathrm{H}$ bond in water as a bond which has a nearly linear $\mathrm{O}-\mathrm{H} \cdots \mathrm{O}$ angle, and a specific range of oxygen-oxygen ( $\mathrm{R}_{\mathrm{OO}}$ ) distances from ca. 2.77-3.5 angstroms. These values of ROO correspond to the distance between the oxygens in the normal ice structure at ${ }^{\circ} \mathrm{C}$, and to the first minimum in the oxygen-oxygen pair correlation function for liquid water, respectively. Since the macroscopic properties of water are constructed through the consideration of microscopic cooperative mechanisms, it is important to explore such dynamics in the condensed phase through simulation, otherwise the properties of the bulk may only be understood in terms of ensemble averaged quantities. Because of the many unusual characteristics of liquid water, and its deviation from theories describing simple liquids, ${ }^{24}$ a molecular understanding is critical in determining its microscopic makeup, and this is attempted through MD simulation.

The simulations of Stillinger and Rahman ${ }^{11,25}$ have sparked a great deal of enthusiasm toward the study of water through MD calculations. This work demonstrates that properties such as pair correlation functions, scattering data, and self diffusion can be adequately reproduced for liquid water by considering the ST2 pairwise additive interaction potential. However, the thermodynamic properties of water have been extremely puzzling for quite some time. For example, heat capacity measurements $\left(\mathrm{C}_{\mathrm{p}}\right)$ of binary $\mathrm{H}_{2} \mathrm{O}_{\mathrm{H}} \mathrm{H}_{2} \mathrm{O}_{2}$ and $\mathrm{H}_{2} \mathrm{O} / \mathrm{N}_{2} \mathrm{H}_{4}$ have shown ${ }^{26}$ that when the mole fraction of water exceeds a value of ca. 0.8 , anomalously large values of $C_{p}$ are observed. At these concentrations, water-water 
interactions are proposed to explain the dramatic deviation from results predicted with models describing simple liquids. These measurements as well as those for the viscosity, 27 thermal expansivity, and isotnermal compressibility 28 properties of water illustrate the thermodynamic singularity in $C_{p}$ at a temperature of $T_{S}=-45^{\circ} \mathrm{C}$. It has been proposed that the dynamic reorientational effects of the $H$-bond in the vicinity of $T_{S}$ correlate to the unusual deviation from simple fluid theory. ${ }^{29}$ For this simulation the $\mathrm{H}$ bond is defined as above. By fitting the ensemble average of the distribution of $\mathrm{H}$-bond lifetimes $\left\langle P(\tau)>\right.$ versus $T$ to a power law of the form $\left(T-T_{S}\right)^{-\alpha}$, a value of $T_{S}=-44+1-5$ ${ }^{\circ} \mathrm{C}$ is determined. Thus it appears that the longer-lived $\mathrm{H}$-bonds in supercooled water near the temperature of $T_{S}$ correlate with the experimentally observed singularity in $C_{p}$. Since the reorientational dynamics mimic tunneling in small clusters from both a temporal and path specific viewpoint (for they are both sensitive to the orientational anisotropy of the IPS), it is critical in developing an understanding of tunneling dynamics in small clusters, for it appears that this aspect of water may provide a direct connection to its bulk phase.

To continue with this discussion, the results of $\mathrm{MD}$ calculations regarding $\mathrm{H}$-bond rearrangement in the water pentamer and hexamer will be considered to further elucidate the possibility of the connection between reorientational dynamics in clusters and liquids. A 6$31 G^{*}$ basis has been used to define an interaction potential at the Hartree-Fock level of ab initio theory, upon which an MD calculation has been conducted. ${ }^{30}$ These simulations were initially tested by adding energy to specific vibrational modes, and monitoring the resulting reorientational dynamics. As expected, energy implanted in librational modes corresponded to $\mathrm{H}$-bond rearrangement, whereas the excitation of the higher energy ring stretching modes resulted in only ring oscillations and no reorientational dynamics were detected. In other words a pathway dependence was determined to be critical. This behavior is not only intuitive, but it can be easily rationalized by the power spectrum obtained in this work. Furthermore, upon study of the addition of energy into the cyclic trimer librations, internal rotations were found to occur through occasional ring opening 
events, resulting in $\mathrm{H}$-bond distuption followed by the subsequent reformation. Although the level of theory used in this calculation is unrefined these results qualitatively describe the minimum energy pathways responsible for internal rotation of the water monomers, in clusters possessing a simple H-bonded network. The VRT tunneling dynamics measured through FIR laser absorption spectroscopy is specifically sensitive to these reorientational pathways.

Finally, quantum tunneling and facile internal rotations in liquid water have been related to several prototypical chemical reactions. Quantum Monte Carlo studies ${ }^{31}$ of the aqueous electron exchange reaction of $\mathrm{Fe}^{3+} \rightarrow \mathrm{Fe}^{2+}$ reveal that quantum tunneling of the solvent directly influenced the free energy of activation for this reaction. A correction to this free energy was included to account for the extra solvation energy due to quantum tunneling modes from the water molecules. The experimentally observed rate of electron transfer was qualitatively reproduced through this correction, and was further tested by examining the isotopic substitution of the solvent molecule $\left(\mathrm{H}_{2} \mathrm{O} \rightarrow \mathrm{D}_{2} \mathrm{O}\right)$. The librational modes of water have also been proposed as a energy source for pushing a reactive pair up and over a reaction barrier. $\mathrm{MD}$ simulations for the $\mathrm{Cl}^{-}+\mathrm{CH}_{3} \mathrm{Cl} \mathrm{SN}$ reaction have been performed in aqueous solution. It was found that in this reaction, energy is applied to the reaction coordinate from the hindered internal rotations of the solvent to permit the barrier crossing to product formation. As a separate consideration, the solvent was also found to be responsible for orientating the reaction partmers to create an ion-dipole complex.

\subsection{Conclusion}

These examples have illustrated that VRT dynamics in liquids and clusters may possibly be treated from analogous perspectives. Although considerable complications arise in the treatment of the liquid phase, an understanding of quantum tunneling in water clusters provides the necessary means for the extrapolation from the cluster to the liquid phase. Furthermore, the study of water clusters may directly pertain to liquid water from 
one final, although intriguing point of view. Several studies have been undertaken which propose that the aqueous phase consists of large aggregates of water molecules which in turn are H-bonded to each other. ${ }^{32-34}$ These studies propose that chlatherates, polyhedral species, or octamers account for the self replicating H-bonded structures which characterize the spacious voids of liquid water, and they rationalize a variety of related thermodynamic properties. Therefore, it will be of enormous value to determine the dynamical and if possible the structural parameters associated with larger water clusters, for if any merit resides in the cluster models of the liquid, these theories will be greatly advanced by FIR VRT characterization. 
1 F. Franks, "Water, A Comprehensive Treatise" (Plenum Press, New York, NY, 1976).

2 D. Eisenberg and W. Kauzmann, The Structure and Properties of Water (OxfordUniversity Press, New York, NY, 1969).

3 F. J. Webster, J. Schnitker, M. S. Friedrichs, R. A. Friesner, and P. J. Rossky, Phys. Rev. Letts. 66, 3172 (1991).

4 S. W. Rick and A. D. J. Haymet, J. Phys. Chem. 94,5212 (1990).; O. A. Karim, P. A. Kay, and A. D. J. Haymet, J. Chem. Phys. 92, 4634 (1990).

5 G. Chalasinski, M. M. Szczesniak, P. Cieplak, and S. Scheiner, J. Chem. Phys. 94, 2873 (1991).

6 G. S. Del Buono, P. J. Rossky, J. Schnitker, J. Chem. Phys. 95, 3728 (1991).

7 R. M. Bentwood, A. J. Barnes, and W. J. Oriville-Thomas, J. Mol. Spectrosc. 84, 391 (1980).; M. F. Vernon, D. J. Krajnovich, H. S. Kwok, J. M. Lisy, Y. R. Shen, and Y. T. Lee, J. Chem. Phys. 77, 47 (1982).; R. H. Page, J. G. Frey, Y. R. Shen, and Y. T. Lee, Chem. Phys. Letts. 106, 373 (1984); D. F. Coker. R. E. Miller, and R. O. Watts, J. Chem. Phys. 82, 3554 (1985);; S. Wuelfert, D. Herren, S. Leutwyler, J. Chem. Phys. 86, 3751 (1987).; A. Engdahl, and B. Nelander, J. Chem. Phys. 86, 4831 (1987).; T. Inoue and S. Kotake, J. Chem. Phys. 91, 162 (1989). Z. S. Huang and R. E. Miller, J. Chem. Phys. 91, 6613 (1989). L. I. Yeh, M. Okumura, J. D. Meyers, J. M. Price, and Y. T. Lee, J. Chem. Phys. 91, 7319 (1989).

8 J. R. Reimers and R. O. Watts, Chem. Phys. 85, 83 (1984).

9 L. H. Coudert and J. T. Hougen, J. Mol. Spectrosc. 130, 86 (1988).

10 C. J. Tsai and K. D. Jordan, J. Chem. Phys. 95, 3850 (1991).

11 W. L. Jorgensen, J. Chandrasekhar, J. D. Madura, R. W. Impey, and M. L.Klein, J. Chem. Phys. 79, 926 (1983). 
12 E. Clementi, W. Kolos, G. C. Lie, and G. Ranghino, Intern. J. Quant. Chem. 17, 377 (1980).

13 J. Caldwell, L. X. Dang, and P. A. Kollman, J. Amer. Chem. Soc. 112, 9144 (1990).

14 H. Saint-Martin, C. Medina-Llanos, and I. Ortega-Blake, J. Chem. Phys. 93, 6448 (1990).

15 F. H. Stillinger and A. Rahman, J. Chem. Phys. 68, 666 (1978).

16 T. A. Weber and F. H. Stillinger, J. Phys. Chem. 86, 1314 (1982).

17 P. Bopp, G. Jancso, and K. Heinzinger, Chem. Phys. Letters 98, 129 (1983).

18 K. Refson, G. C. Lie, and E. Clementi, J. Chem. Phys. 87, 3634 (1987).

19 M. J. Frisch J. E. Del Bene, J. S. Binkley, and H. F. Schaefer,III, J. Chem. Phys.84, 2279 (1986).

20 R. D. Amos, Chem. Phys. 104, 145 (1986).

21 C. E. Dykstra, J. Chem. Phys. 91, 6472 (1989).

22 D. J. Swanton, G. B. Backay, and N. S. Hush, Chem. Phys. 83, 69 (1984).

23 J. C. Owicki, L. L. Shipman, and H. A. Scheraga, J. Phys. Chem. 79, 1794 (1975).

24 Simple liquids exhibit close packing which results in 10-15 nearest neighbors within the first solvation shell, however, water has a very low number of nearest neighbors (4-5), and is full of spacious voids.

25 F. H. Stillinger and A. Rahman, J. Chem. Phys. 60, 1545 (1974).

26 M. Oguni and C. A. Angell, J. Chem. Phys. 73, 1948 (1980).

27 B. L. Halfpap and C. M. Sorensen, J. Chem. Phys. 77, 466 (1982).

28 H. Kanno and C. A. Angell, J. Chem. Phys. 73, 1940 (1980).

29 F. Sciortino, S. L. Fornilli, J. Chem. Phys. 90, 2786 (1989); F. Sciortino, P.

H.Poole, H. E. Stanley, and S. Havlin, Phys. Rev. Letts. 64, 1686 (1990).

30 P. L. M. Plummer, J. Mol. Struct. 237, 47 (1990).

31. J. S. Bader, R. A. Kuharski, D. Chandler, J. Chem. Phys. 93, 230 (1990). 
32 F. H. Stillinger, Science 209, 451 (1980).

33 R. J. Speedy, J. Phys. Chem. 88, 3364 (1984).

34 S. W. Benson and E. D. Siebert, J. Amer. Chem. Soc. 114, 4269 (1992). 


\section{Chapter 2}

\section{The Far Infrared Laser Spectrometer}

\subsection{Experimental Introduction}

Over the past several years, the Berkeley tunable FIR laser spectrometer has been described in several review papers ${ }^{1,2}$ and dissertations, $3,4,5,6,7$ therefore, only a brief description of the entire apparatus will be included in this chapter. There are several purposes for this chapter. First it is to serve as a sort of technical manual so that future experimenters will have a starting point from which they may work. Since this work represents the first data obtained in the spectral region above $2.4 \mathrm{THz}$, this chapter will include procedural details that have been determined to be useful for the operation of the spectrometer at these frequencies. For example, it will become apparent that the presently available laser coverage is rather sparse above $3 \mathrm{THz}$, so part of this chapter will include new spectrometer features which extend the microwave coverage about a particular laser. Also, the description and specifications for Ge:Ga photoconductors will be presented, for these are the detectors of choice for high frequency scanning. Finally, helpful suggestions along with possible sources for specification improvements, will be discussed separately for each component of the apparatus.

The apparatus is briefly described as follows and reference should be made to Figure 2.1 for the general layout of the FIR laser system. A 150 Watt line tunable $\mathrm{CO}_{2}$ 
Figure 2.1

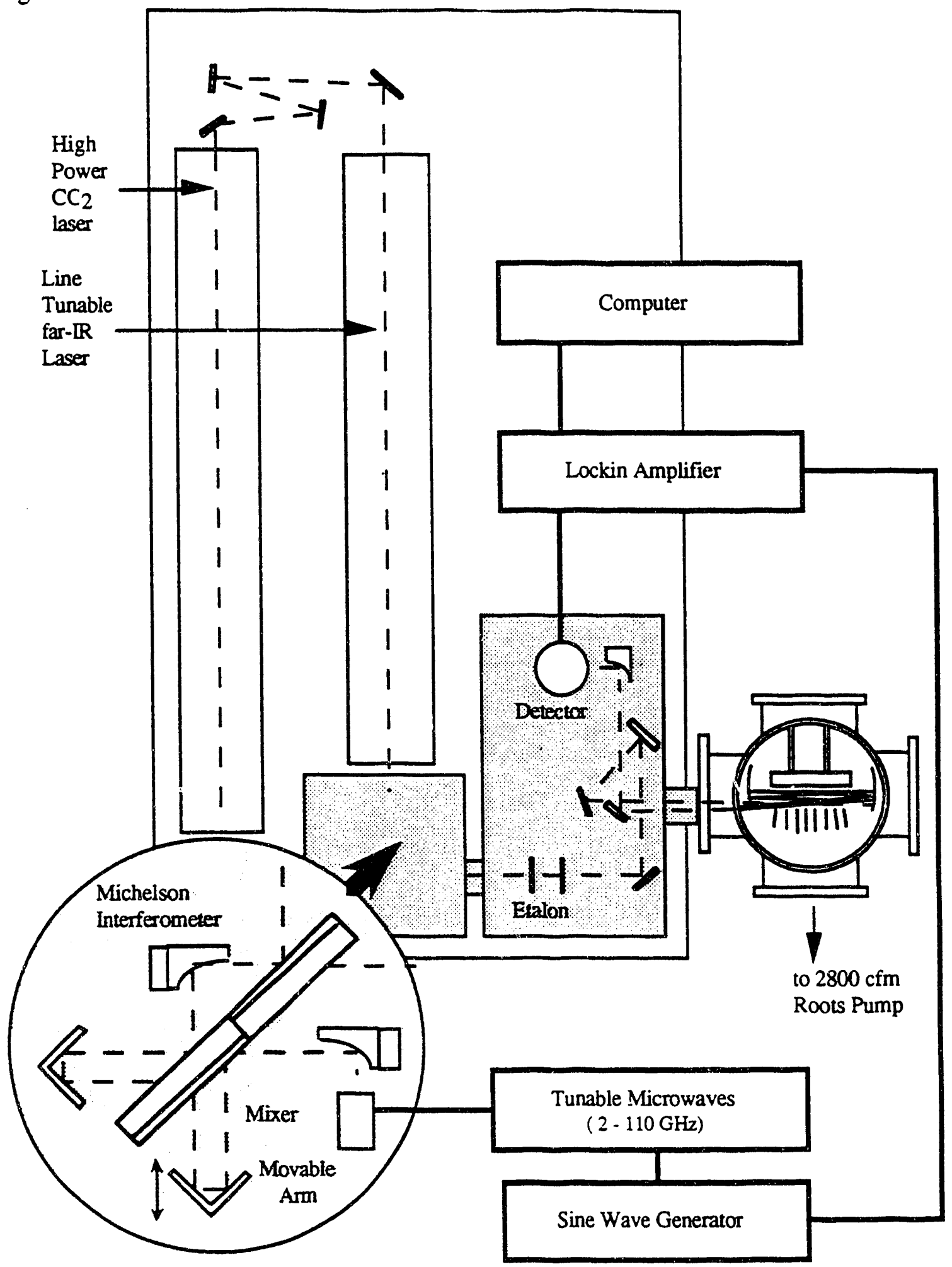

The Berkeley tunable FIR laser absorption spectrometer. 
laser is used to longitudinally pump a fixed frequency FIR laser which acts as the local escillator (LO) or carrier frequency. Tunable FIR radiation is generated by mixing continuousiy tunable ( $2-110 \mathrm{GHz}$ ) microwave radiation (the intermediate frequency: IF) withi the fixed frequency LO in a Schottky barrier diode mounted in a corner cube mixer. These tunable sideband frequencies $\left(v_{\text {sidebands }}=v_{\text {laser }}+/-v_{\text {microwaves }}\right)$ are separated from the more intense $\mathrm{LO}$ ( $\mathrm{ILO}_{\mathrm{LO}}=\mathrm{I}_{\text {sidebands }} \times 10^{2}-10^{4}$ )with a Martin-Puplett diplexer for which the optical path is shown in the circular blowup of Figure 2.1. Further separation is routinely accomplished with two Ni meshes operating as a tunable Fabry-Perot etalon. The shaded region of Figure 2.1 represents a purge box through which the FIR laser beam proceec's toward the chamber. A contriuous flow of dry $\mathrm{N}_{2}$ or $\mathrm{Ar}$ is supplied as a purge so that scanning near FIR amostheric water absorptions is made possible. Once entering the chamber, the raciation is nultipassed ${ }^{3} 8-16$ times through a supersonically cooled molecular expansion, from which the clusters of interest are formed. Typical chamber pressures of 20-300 mtor: are maintained bv a $2800 \mathrm{cfm}$ Roots blower system, depending on the characteristics of the source. The laser radiation is detected by a variety of FIR detectors, and atsorption features are measured as a decrease in detected sideband power. The tunable sicebands are frequency modulated at $50 \mathrm{kHz}$ with a sine wave, and absorption signals are processed with !ockin detection at $2 f$. This scheme typical yields a sensitivity specification for which the minimum detectable fractional absorption is nominally $1-5 \mathrm{ppm}$.

\subsection{The Apollo $\mathrm{CO}_{2}$ Laser}

The detailed discussion of the tunable FIR laser absorption spectrometer begins with the continuous wave (cw) carbon dioxide infrared laser, ${ }^{8}$ which is used to optically prepare the FIR gain medium into an inverted lasing energy level. The model used is an Apollo 150 (currently supported by Laser Photonics Inc.) which can be tuned over approximately 80 frequencies in the $10.6 \mu \mathrm{m}$ region of the infrared. The laser is designed so that both laser end mirrors are mounted intracavity to maximize the output power. The 
grating (APOLLO PN A379-009) acts as a totally reflective end mirror and is ruled on a copper substrate with a 135 line/inch groove spacing. It is efficiently heat sunk to the cooling plate with a small amount of DOW Corning Heat Sinking Compound. The ZnSe output coupler (II-VI PN 656-123) is designed with a 20 m radius of curvature on the intracavity side of the optic, upon which an $80 \%$ reflective dielectric $10.6 \mu \mathrm{m}$ coating is applied. This dielectric serves as a partially reflective laser end mirror. On the flat output side of the optic, a $100 \%$ antireflective dielectric $10.6 \mu \mathrm{m}$ coating is applied to assure the complete transmission of the intense IR radiation. When aligned properly, this cavity configuration supports a Gaussian output beam of $12 \mathrm{~mm}$ in diameter, for which a maximum cw output power of ca. 130 Watts is maintained when operating at 10P(20) with $100 \%$ fresh gas, and $60 \mathrm{~mA}$ through each arm of the laser.

The Apollo laser has had a variety of problems over its period of operation and some solutions related to its repair are outlined below, for undoubtedly it will need attention some time in the future. To avoid serious problems, this discussion will begin with a few simple steps which may be taken to prolong its operational lifetime. The gas mixture of $6 \%$ $\mathrm{CO}_{2}, 18 \% \mathrm{~N}_{2}$, and $76 \% \mathrm{He}$ is flowed through the two arms of the laser at the output coupler and grating ends, and is evacuated through a center tee which connects the two discharge tubes. As a cost effective measure, the gas mixture is directed from the vacuum pump exhaust into a $\mathrm{CO}_{2}$ gas recycling unit which removes residual oil from the mixture and reintroduces it into the laser through a tee in the gas input plumbing. The $\mathrm{CO}_{2}$ laser is generally operated continuously for long periods of time, therefore it is imperative that the gas recycler be maintained so that the intracavity optical components will be protected from the possibility of oil contamination. Such an occurrence will most certainly reduce the performance of the laser, and has the potential for permanently destroying the intracavity optics. The recycler has recently been upgraded so that it meets the standards neeced to keep the laser functioning properly and a diagram of this unit along with a list of 
replacement part numbers, is contained in the FIR laboratory notebook. Possibly the most critical component regarding the general maintenance of the recycler is the oil indicating filter, because it provides a measure of the amount of oil entering the discharge region of the laser. It should be checked as often as every 2-3 days to assure that the entire unit is operating properly. The majority of the oil from the pump exhaust is trapped in two oil filter reservoir boils. If these are emptied when the accumulation of oil is substantial, the oil indicating filter should last for quite some time. A catalytic converter is used to replenish the $\mathrm{CO}_{2}$ gas from $\mathrm{CO}$ which is generated as a discharge byproduct. The catalyst in this unit must be changed (40 grams) every 6-12 months.. The oven which houses the catalyst should always remain on because it takes several hours for it to reach its operational temperature. By checking these components regularly, the performance characteristics of the $\mathrm{CO}_{2}$ laser will be sustained.

Another routine maintenance task involves the high voltage electrodes located in the cathode housings at the ends of the center tee. When striking the discharge a transient voltage at the cathodes rises to a level between $20-25 \mathrm{kV}$, after which they are continuously maintained at $6 \mathrm{kV}$ to keep the discharge tubes lit. Each housing is filled with a high dielectric oil to keep the cathodes thermally stable, and this oil must completely surround the entire metal portion of the electrode to prevent electrical breakdown. Each housing seals against a mounting plate on the laser with $\mathrm{O}$-rings, and because of day-to-day thermal cycling, these seals tend to leak oil at a rather slow rate. Therefore, it is important to check the oil level every $20-30$ days. If this task is forgotten, and the oil in these housings drops below a critical level, the self destruction of the cathode housings will occur. Thus, checking this oil level regularly is highly recommended.

The tube nearest the grating has been particularly problematic over the past few years. The most common failure has been the destruction of the O-ring seals which isolate the discharge from the rest of the laser fluids. When these seals are broken the risk of 
leaking oil and/or water into the discharge tubes is high, and this almost certainly leads to damaged laser optics. If any vacuum problems, low output power, or any amount of oil in the discharge tube is noticed, the cathode housings should be considered as a possible source of the problem. If the diagnosis is related to the electrodes, it is most likely that the glass laser tubes must be removed from the housings. This process is begun by draining the water and cathode oil from the laser tubes and removing all of the panels of the laser. With the cathode housing locked in place, the endmounts are slowly pulled away from the tubes while the water jacket is gently twisted. The tubes are then carefully removed and stored in a safe place. Replacing the tubes is a more difficult task because they must pass through the $\mathrm{O}$-ring seals in the cathode and anode ends of the laser. A small amount of vacuum grease should be applied to each O-ring through which the tube must pass, and the tube should be twisted into position while applying the smallest amount of force required to the endblock to get the tube through the seals. When doing this it is critical to be certain that the tubes are not entering the housings at an angle, otherwise the force exerted on the metal endblock will crack the glass. Regardless of the amount of care given, this procedure usually amounts to cracking one of the tubes about $50 \%$ of the time, so it is always useful to have an extra tube in the laboratory.

It should be noted that when turning the $\mathrm{CO}_{2}$ laser on at the beginning of the day, the diode should always remain disconnected from any electrical device, such as the microwave source or the bias box. Ground spikes and an excessive amount of RF have both been the cause of many diode contact problems in the past when striking the $\mathrm{CO}_{2}$ discharge. In tact RF noise was found to be completely prohibitive when initial experiments conducted with small anode diodes such as the 1T12. The problem was found to originate in the high voltage cabling which is submerged in the cathode oil baths. In the past the cathode oil had a tendency to wick along the multistrand high voltage conductor (under the insulation of the cable) until it worked its way back to the power supply. Over a 
long period of time, a large amount of oil buiit up under the insulation and a discharge path for the high voltage was created within the cable so that they essentially became an experimentally (prohibitively noisy) RF source. As a result, the faster more sensitive 1T12 diode would consistently burn out. To solve this problem the cables were replaced, and in doing this the cable connection to the cathode was modified to prohibit the wicking of oil along the high voltage conductor. This modification is shown below in Figure 2.2.

Figure 2.2

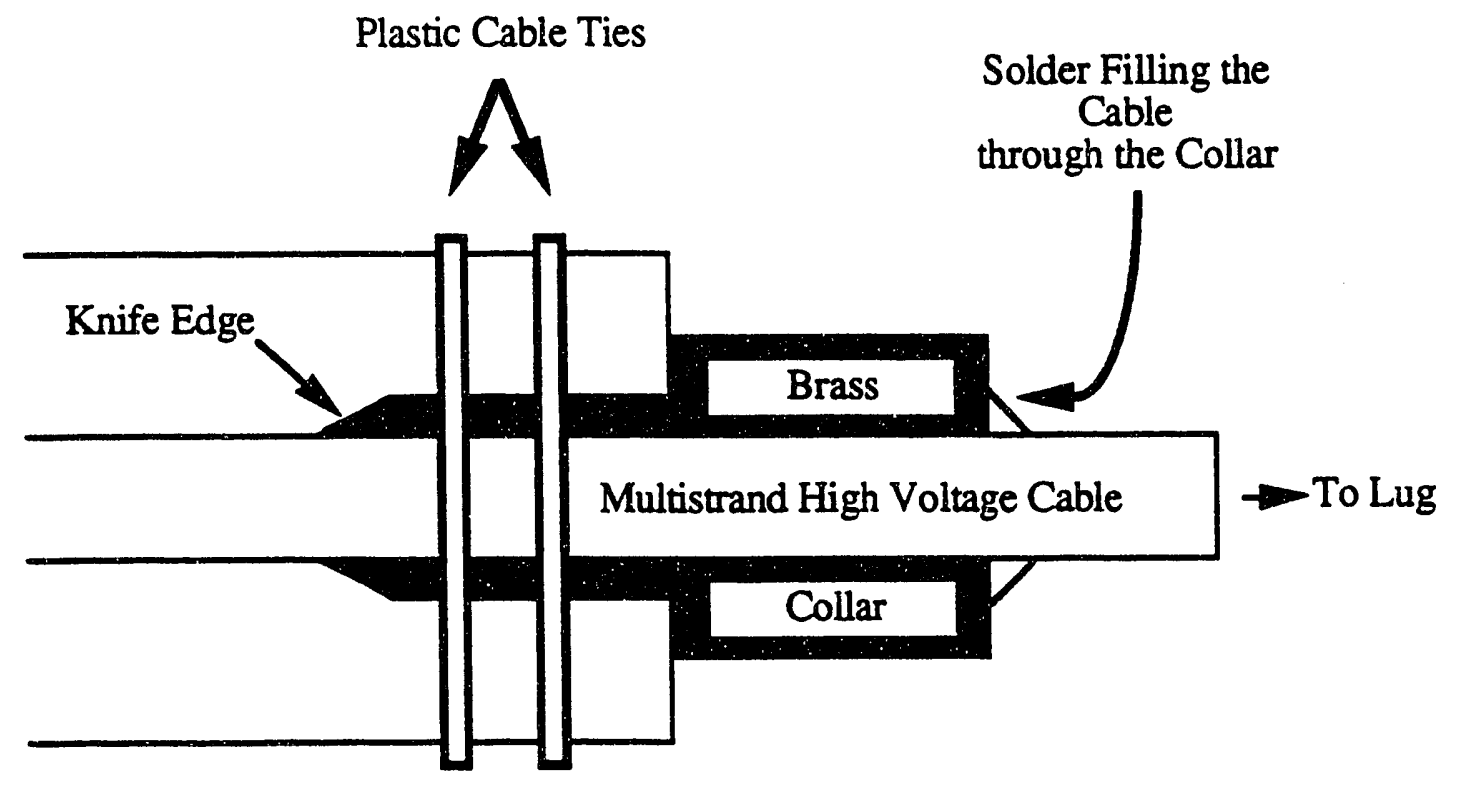

The modified cable connection to the cathode electrodes.

The brass collar in this illustration has an I.D. which is slightly larger than the O.D. of the cable, and a knife edge was machined so that it could easily cut under the insulation of the cable. Once in place, the collar was filled with solder to essentially create a solid core conducting wire, and plastic tie wraps were used to tightly compress the insulation around the brass collar. A lug was then soldered onto the bare wire and the connection was screwed tightly into the cathode to maintain good electrical contact. The replacement of 
these cables provided for a quiet laser which was essential in operating the more sensitive diodes, and no RF noise has since been noticed after the modification.

As previously mentioned, any major repair of the laser usually requires disassembling the discharge tubes. If the cathode housings are removed it is almost always necessary to realign the laser tubes relative to each other, and the intracavity optical components, because there is a fair amount of play in the positioning of the four independent electrodes. Therefore, it is important to adjust their centers so that they reside along a unique laser axis. To do this it is recommended that the anodes be aligned to the center of the mounting plates first. This assures that the ends of the tubes are centered relative to the grating and output coupler. After this, the cathode housings are tapped with a mallet into alignment. To check the quality of the alignment, the intersection of the centering crosshairs of an autocollimating site $^{9}$ are positioned at the center of the tubes in the region of the four electrodes.

Although many problems have been presented above, the operation of the $\mathrm{CO}_{2}$ laser is generally straightforward. The overall system is more or less reliable and requires only minor adjustments from day to day. If the power output is optimized daily, the alignment into the FIR laser is occasionally checked, (while AM-ing the laser system with a low frequency mechanical chopper, and detecting the radiation with the Schottky diode) and the general maintenance suggestions are followed, the laser will perform in a reliable fashion.

\subsection{The Far Infrared Laser}

The far infrared waveguide laser is very similar to the design of Farhoomand et al., ${ }^{10}$ and a detailed description of the laser used throughout this work can be found in $\mathrm{K}$. B. Laughlin's Dissertation. ${ }^{7}$ An excellent review of FIR waveguide lasers can also be found in the articles by Degnan ${ }^{11}$ and Abrams and Chester. ${ }^{12}$ In Table 2.1, the lasers which have been observed for frequencies greater than $2.4 \mathrm{THz}$ are reported. Briefly, the 
Table 2.1

Laser lines that may be used for scanning above 2.4 Thz. With the exception of the 109 $\mu \mathrm{m} \mathrm{CH} \mathrm{CH}_{2}$ laser, all frequencies have been observed with both a 6 and $10 \mathrm{~mm}$ output coupler. Rectification values are reported after purging atmospheric water from the FIR beam path. The polarization of the lasers are referred to the vertically polarized Apollo $\mathrm{CO}_{2}$ laser. The $2633900 \mathrm{MHz}{ }^{13} \mathrm{CH}_{3} \mathrm{OH}$ laser has been calibrated in this laboratory, and this results in a frequency precision which is slightly worse than the other lasers. Although the laser near $89 \mu \mathrm{m}$ has been observed, at this time its frequency is not known to better than three significant digits.

\begin{tabular}{l|l|l|l|l|l}
\hline Laser Frequency & Wavelength & Medium & Pump & Polarization & Rectification \\
\hline \hline $2409293.2 \mathrm{MHz}$ & $124 \mu \mathrm{m}$ & $\mathrm{CH}_{2} \mathrm{DOH}$ & $10 \mathrm{P} 34$ & PAR & $100 \mathrm{mV}$ \\
$2447968.5 \mathrm{MHz}$ & $122 \mu \mathrm{m}$ & $\mathrm{CH}_{2} \mathrm{~F}_{2}$ & $9 \mathrm{R} 22$ & PRP & $200 \mathrm{mV}$ \\
$2522781.5 \mathrm{MHz}$ & $118 \mu \mathrm{m}$ & $\mathrm{CH}_{3} \mathrm{OH}$ & $9 \mathrm{P} 36$ & PRP & $2000 \mathrm{mV}$ \\
$2546495.0 \mathrm{MHz}$ & $117 \mu \mathrm{m}$ & $\mathrm{CH}_{2} \mathrm{~F}_{2}$ & $9 \mathrm{R} 20$ & PRP & $500 \mathrm{mV}$ \\
$2588361.8 \mathrm{MHz}$ & $115 \mu \mathrm{m}$ & $13 \mathrm{CH}_{3} \mathrm{OH}$ & $10 \mathrm{R} 16$ & PAR & $350 \mathrm{mV}$ \\
$2633900 \mathrm{MHz}$ & $114 \mu \mathrm{m}$ & $13 \mathrm{CH}_{3} \mathrm{OH}$ & $9 \mathrm{P} 30$ & PAR & $800 \mathrm{mV}$ \\
$2664058.3 \mathrm{MHz}$ & $113 \mu \mathrm{m}$ & $\mathrm{CH}_{2} \mathrm{DOH}$ & $9 P 12$ & PAR & $200 \mathrm{mV}$ \\
$2714715.1 \mathrm{MHz}$ & $110 \mu \mathrm{m}$ & $13 \mathrm{CH}_{3} \mathrm{OH}$ & $10 \mathrm{R} 18$ & PAR & $200 \mathrm{mV}$ \\
$2742946.0 \mathrm{MHz}$ & $109 \mu \mathrm{m}$ & $\mathrm{CH}_{2} \mathrm{~F}_{2}$ & $9 P 24$ & PAR & $350 \mathrm{mV}$ \\
$2851169.2 \mathrm{MHz}$ & $105 \mu \mathrm{m}$ & $13 \mathrm{CH}_{3} \mathrm{OH}$ & $10 \mathrm{OR} 18$ & PAR & $150 \mathrm{mV}$ \\
$2907088.9 \mathrm{MHz}$ & $103 \mu \mathrm{m}$ & $\mathrm{CH}_{3} \mathrm{OD}$ & $9 P 30$ & PRP & $50 \mathrm{mV}$ \\
$3105936.8 \mathrm{MHz}$ & $96 \mu \mathrm{m}$ & $\mathrm{CH}_{3} \mathrm{OH}$ & $9 R 10$ & PAR & $300 \mathrm{mV}$ \\
$3630000.0 \mathrm{MHz}$ & $89 \mu \mathrm{m}$ & $\mathrm{CH}_{3} \mathrm{OD}$ & $9 P 30$ & PAR & $200 \mathrm{mV}$ \\
\hline $4251673.8 \mathrm{MHz}$ & $70 \mu \mathrm{m}$ & $\mathrm{CH}_{3} \mathrm{OH}$ & $9 P 34$ & PRP & $100 \mathrm{mV}$ \\
\hline
\end{tabular}


$\mathrm{CO}_{2}$ laser is focussed with a $1 \mathrm{~m}$ focal length mirror and is folded into the FIR laser with a series of flat mirrors which help to minimize spherical aberration of the Gaussian pump beam. The infrared beam is then directed through a $10.6 \mu \mathrm{m}$ AR coated flat 0.75 inch diameter, ZnSe input window (CVI PN PP0708-ZS) mounted on a port in the vacuum tight stainless steel laser endblock. The beam proceeds into the laser cavity through a $4 \mathrm{~mm}$ hole in a gold coated flat input coupler (CVI PN HR-CU-2.0-AU) which serves as a fixed laser end mirror. From this point the $\mathrm{CO}_{2}$ laser pump beam slowly diverges until it is totally absorbed at approximately $2 / 3$ the round trip length of the FIR laser cavity. The output coupler is mounted on a translational stage driven by a 0.5 " travel motorized encoder micrometer (ORIEL PN 18219) so that coarse adjustments to the cavity length are possible. A water cooled electro-mechanical translator (EMT), which has a travel of approximately $60 \mu \mathrm{m}$ over its $0-40 \mathrm{~V}$ dynamic range, is mounted at the rear of the output coupler to allow for fine tuning of the laser cavity. Both end mirrors are water cooled to provide a heat sink for the powerful $\mathrm{CO}_{2}$ pump beam incident on the intracavity optics. The leak rate of the laser is currently ca. 500 mtorr/day, which is low enough to use lasers which require expensive lasing media without the need to flow the gas. The most likely place where leaks are found is in the end mirror cooling lines, and such problems are avoided by continuously flowing water through this plumbing. External adjustments of the horizontal and vertical positions of the end mirrors is made possible through vacuum tight electrical feedthroughs which connect to precision 0.5" motorized micrometer (ORIEL PN 18030) drives. When properly aligned the optimum output mode of the laser should be the $\mathrm{EH}_{11}$ mode; this is the lowest order hybrid mode for a waveguide laser of this kind. ${ }^{12}$

For the experiments presented in this dissertation a $6 \mathrm{~mm}$ diameter FIR output coupler is used to minimize cavity losses, thus allowing weak lasers to more easily surpass gain threshold. This cavity configuration has increased the power output of several lasers which were previously used in this spectral region, and has permitted the use of many others, as can be realized by the inspection of Table 2.1. Nevertheless a variety of output 
couplers have been tested in this laser system. The ones most commonly used have clear apertures of 6,8 , or $10 \mathrm{~mm}$ drilled through a gold coated flat copper end mirror to allow for the radiation to be coupled out of the gain medium. The infrared beam incident on the output hole is reflected back into the laser cavity with a flat piece of Z-cut quartz with a silicon overlayer (CVI PN PP-2020SI) placed behind the output coupler. This prevents the heating of the laser endblock and output window by the $\mathrm{CO}_{2}$ pump radiation. Output coupling of this kind has worked reasonably well, and has been the standard method used with this laser system. However, because the Si disk which reflects the pump beam is positioned several millimeters away from the Au coated reflector, and because it is not a perfectly transmitting optical element with respect to the FIR radiation, the possibility for two separate FIR longitudinal modes always exists. The competition of these cavity modes may in fact be the cause for lasing instabilities. For this reason, hybrid output couplers have been constructed out of a $4 \mathrm{~mm}$ thick flat piece of $\mathrm{Si}$ with a $\mathrm{Au}$ overlayer to try and address this problem. For this optical component, a 6-8 mm aperture was created in the center of the Si disk, so that the bare substrate would allow for transmission of the FIR laser beam. These hybrid couplers were manufactured at Berkeley, with the Majda bell jar metal deposition chamber on the 3rd floor of Hildebrand Hall. The apertures were masked from the metal deposition beam with a circular piece of iron which was held in place with strong bar magnets placed on top of the Si disks. After allowing the chamber to reach ca. 5 $x 10^{-7}$ torr, $5 \mathrm{~nm}$ of chromium was deposited onto the disks to assure that the Au overlayer would adhere to the Si substrate, after which $660 \mathrm{~nm}$ of $\mathrm{Au}$ was deposited. This thickness corresponds to nearly 5 skin depths at $300 \mathrm{GHz}$ and provides for good reflectivity throughout the entire FIR spectral region. Because the gold overlayer is thin compared to the wavelength of the FIR lasers, such a design should support only one cavity mode for a given position of the translating output coupler. After installing the $8 \mathrm{~mm}$ element, it performed well for some period of time with high output powers, however its performance seemed to degrade with time presumably due to a slight absorbance at the infrared pump 
frequency. Upon inspection of the $\mathrm{Si}$, it appeared that the material had been heat treated which seemed to scar or aneal the uncoated area. In the future, Ultra high purity silicon, which can be purchased from Lattice Material Co., should be used for this application to avoid the slow degradation of this type of optic.

Typically the FIR laser is extremely reliable, so in the event that the power output seems low the alignment of the far infrared laser should only be attempted after all other possibilities have been considered. Occasionally the laser does drift out of alignment over a period of time, so a description of the alignment procedure is included for when this situation arises. The alignment of the laser is, in principle, easy to understand yet often difficult to apply. The final result should yield two end mirrors parallel to one another and perpendicular to the laser bore axis. To achieve this, alignment apertures which are stored under the output endblock of the laser are used to help guide a HeNe laser beam through the center of the waveguide tube. After removing both of the end mirrors from the Gimbal mounts, the two alignment apertures are bolted onto the input and output window ports of the laser endblocks so that the holes which lie off center define a line parallel to the laser axis. The HeNe laser is then passed through an adjustable telescope which is used to focus the beam in the far field. With two mirrors, the HeNe is directed through the output endblock and is passed through the center holes of the two alignment apertures. The HeNe must then be walked towards the two holes which lie off of center on the alignment apertures. Once this is accomplished, the input coupler is installed and the motorized micrometers are adjusted to send the HeNe reflection back onto itself through the first hole and onto the telescope leaving the input coupler aligned.

To align the output coupler, the direction of the HeNe is reversed so that it passes through the input endblock and is centered through the two center holes of the alignment apertures. The output coupler is then installed and the reflected beam off of the Si disk is positioned onto the initial hole leaving the output coupler aligned. ...now for the hard part! To insure that the optics are aligned relative to each other, a fine adjustment of the cavity 
Figure 2.3
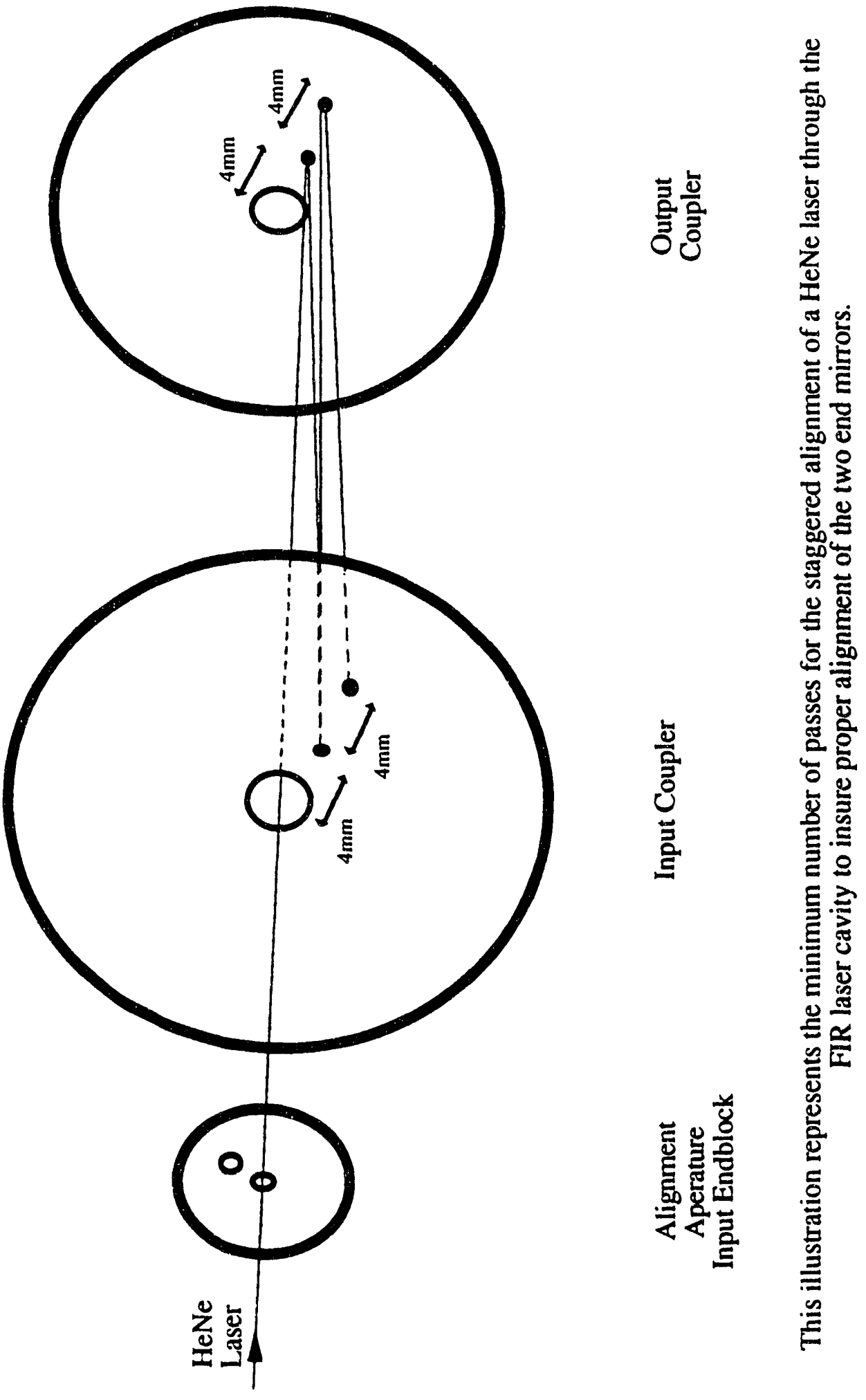
end mirrors must be conducted. With the two end mirrors in place, the HeNe must be walked off of the center of the output coupler, (ca. $4 \mathrm{~mm}$ for the $6 \mathrm{~mm}$ output coupler and ca. $6 \mathrm{~mm}$ for the $10 \mathrm{~mm}$ ) so that it is imaged onto the gold coating of the laser optics. This spot observed on the Au overlayer of the output coupler must remain vertically centered during this adjustment, while the HeNe laser continues to pass through the center hole of the input alignment aperture. This orients the beam so that it is no longer parallel to the laser tube axis. This is illustrated in Figure 2.3. By moving the output coupler, the beam may then be positioned onto the input coupler in a similar manner. Iterating between the two mirrors will create the back-and-forth off-axis pattern that is shown in Figure 2.3. The separation of each of the spots on the end mirrors may be measured by inserting a clear "transparency" between the laser tube and each mirror. After this process is completed, only minor adjustments of the cavity will be needed to optimize the power output of the laser.

Often times, the realignment of the FIR laser provides an opportune time to adjust the $\mathrm{CO}_{2}$ laser input into, and through the FIR laser bore. This should be done any time before the final installation of the output coupler. To do this, all apertures should be removed from the endblocks and the cooling water to the end mirrors and the laser cooling jacket must be flowing. The $\mathrm{CO}_{2}$ laser is then turned on with a low current setting and a power measurement should be taken before imaging it into the FIR cavity. The IR is then centered on each of the gold coated copper mirrors which fold the light toward the FIR laser and is carefully adjusted so that it passes through both the center of the input window port and the $4 \mathrm{~mm}$ hole in the input coupling optic. The $\mathrm{CO}_{2}$ laser power meter is then mounted at the FIR laser output port and the incident power should be optimized at this point with the final two folding mirrors while maintaining the focussed IR in the center of the $4 \mathrm{~mm}$ input hole. The IR power density is extremely high at the $4 \mathrm{~mm}$ hole of the input coupler. thus, a great deal of caution is recommended when monitoring the beam at this 
point. This should be done with a small piece of cinder block. and protective eveware should definitely be worn!

Once aligned the FIR laser power should be measured with the Scientech (Scientech PN 360001 S/N 1927) power meter at the highest frequency line available because the position of the optics is obviously most sensitive to the shorter wavelength lasers. As a benchmark, the $118 \mu \mathrm{m} \mathrm{CH} \mathrm{CH}_{3} \mathrm{OH}$ laser with the 6 and $10 \mathrm{~mm}$ output couplers installed in the laser provide power output levels of ca. 150-200 and 250-300 mW, respectively, when pumping the cavity with $100 \mathrm{~W}$ at $9 \mathrm{P} 36$. If this is not the case, small adjustments of the end mirrors should be attempted while monitoring the laser power for its optimum output. The time constant of this power meter is slow, (ca. $3 \mathrm{~s}$ ) so adjustments need to take place patiently.

\subsection{The Schottky Barrier Diode}

The GaAs Schottky Barrier Diode is without question the most important element of the tunable FIR laser spectrometer. Fortunately, technological advances in the microfabrication of these elements evolve at an extremely rapid pace. ${ }^{13}$ To date these elements remain the most sensitive mixing devices for generating sum and differences frequencies in the spectral range accessible with optically pumped FIR lasers (0.4-5.0 Thz.), however other devices have been characterized for this purpose. ${ }^{14}$ This section attempts to summarize the present state of GaAs diode technology used with the Berkeley FIR lasers.

This discussion begins with a brief summary of the important characteristics for Schottky diodes so that an understanding of the mixers may be applied to the improvement of the tunable FIR apparatus. The most serious signal losses in a Schottky diode occur at three places in the diode circuitry; between the antenna and mixer, within the mixer itself, and in the coupling of the IF onto the element. Although the corner cube open structure design, originally proposed by Krautle et al..$^{15}$ is rather inefficient, it is still the most 
effective way to couple the LO and RF with the diode via a contacted antenua. Also, for the purposes of the tunable FIR laser system, the coupling of the IF onto the diode is usually not an issue, for although impedance mismatching problems lead to power fluctuations at the element, the available microwave sources in the laboratory can provide sufficient power from approximately $2-90 \mathrm{GHz}$. This leaves the diode itself as the primary source for technological improvement.

Since the diode noise ${ }^{16}$ is far below other noise sources of the experiment, (detector or laser) it will not be considered as a means for improving the laser system. Thus, the optimization of diode performance for this experiment involves the enhancement of mixer conversion efficiency at higher FIR laser (LO) frequencies. The figure of merit relevant to this aspect of performance is known as the diode cutoff frequency, which should ideally be at least $3 X$ the frequency of the LO. This frequency is defined as $v_{c o}=\left(2 \pi R_{s} C_{j 0}\right)^{-1}$, in which $R_{S}$ is the series impedance and $C_{j o}$ is the junction capacitance of the contacted diode. These two terms are referred to as the parasitics inherent in the diode which reduce the responsivity of the element at higher freç' lencies, and are characterized by the following equivalent circuit $^{16}$ shown in Figure 2.4.

Figure 2.4

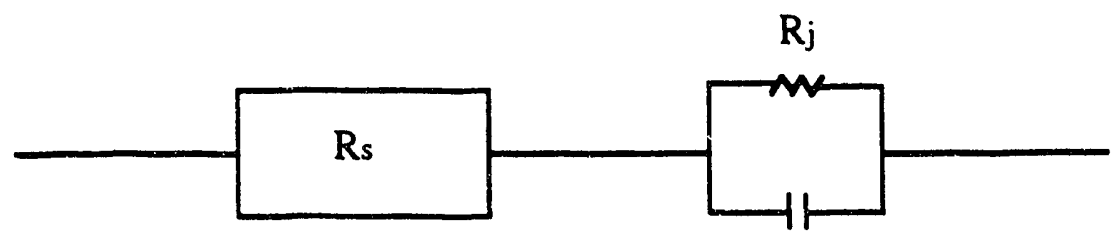

Cjo

This diagram represents the equivalent circuit for a contacted diode.

The minimization of the $\mathrm{R}_{\mathrm{s}} \mathrm{C}_{\mathrm{j} 0}$ product can be accomplished by increasing the dopant concentration and reducing the size of the contact rea to sufficiently match the impedance 
that the diode secis from the antenna. A great deal of effort has been and continues to be devoted to this area of research.

The 1T12 and 1T13 diodes recently purchases from the Crowe group at the University of Virginia (UVa) are exceedingly good examples of performance improvements in this field of research. Unfortunately, the available time period only allowed for the testing of the 1T12 element and the majority of the discussion to follow compares its performance to rnat of the 117 dicde. The following test data has been supplied by UVa for the two new diodes, and are reported in Table 2.2.

Table 2.2

Specifications for the two $0.5 \mu \mathrm{m}$ anode diameter diodes. Each parameter is defined in the text.

\begin{tabular}{l|l|l}
\hline Specification & $1 \mathrm{~T} 12$ & 1113 \\
\hline \hline & & \\
\hline \hline$C_{j 0}=$ & $0.5 \mathrm{fF}$ & $0.7 \mathrm{fF}$ \\
$\mathrm{R}_{\mathrm{S}}=$ & $24.3 \Omega$ & $15 \Omega$ \\
$\Delta \mathrm{V}=$ & $78.6 \mathrm{mV}$ & $76.9 \mathrm{mV}$ \\
$\mathrm{V}_{\mathrm{KNEE}}=$ & $0.78 \mathrm{~V}$ & $0.77 \mathrm{~V}$ \\
$\mathrm{~V}_{\mathrm{BR}} @-1 \mu \mathrm{A}=$ & $5.4 \mathrm{~V}$ & $5.1 \mathrm{~V}$ \\
\hline
\end{tabular}

The parameters $C_{j 0}$ and $R_{S}$ have already been defined above, and the product of the two yield a cutoff frequency of 13.1 and $15.2 \mathrm{THz}$ for the $1 \mathrm{~T} 12$ and $1 \mathrm{~T} 13$ diodes, respectively. $\Delta \mathrm{V}$ is the change in voltage across the diode from -10 and $-100 \mu \mathrm{A}, \mathrm{V}_{\mathrm{KNEE}}$ is the voltage of an adequately contacted diode at zero bias and $V_{B R}$ is the limit of the video response of the element at which the diode breaks down.

Video esponse can be used to describe the performance of a diode, and is measured as the ratio of rectification (in volts) to the incident laser power (in Watts). This figure of merit has been defined by accurately measuring the power of the FIR laser with the Scientech 360001 power meter and then imaging the radiation onto the corner cube 
receiver to detect as diode rectification. The video response for the 1 T12 diode at 432 and $118 \mu \mathrm{m}$ was measured to be approximately 300 and $50 \mathrm{~V} / \mathrm{W}$, for laser powers of 10 and $100 \mathrm{~mW}$, respectively. Clearly the diode is a better receiver at the lower frequencies, however the $118 \mu \mathrm{m}$ value is nearly a factor of three larger than that for the 117 element at this frequency as measured in this laboratory. These results compare well with those reported by Titz et al. ${ }^{17}$ Also, the single sideband conversion efficiency at $2.5 \mathrm{THz}$ exhibited approximately a five-fold increase for the 1T12 over that of the 117 , as measured by a Ge:Ga stressed photoconductor while using a 750 line/inch $\mathrm{Ni}$ mesh Fabry-Perot etalon to isolate the pure sideband radiation. Although there are many sources for error in measurements of this kind, these data qualitatively show that the 1T12 outperforms the 117 diode at frequencies $>2.4 \mathrm{THz}$ and further research should be conducted to characterize this element with the Berkeley tunable FIR laser system.

The 1T12 diode is without question the highest quality diode used thus far with the tunable FIR laser system, yet it is not without its drawbacks. The diameter of the contact area for the faster diode is only $0.5 \mu \mathrm{m}$ which is a factor of 2 smaller than that of the 117 , and this makes the diode more difficult to contact and more sensitive to transient voltage surges in the laboratory. Photographs of these diodes are shown in Figure 2.5 as supplied by T. W. Crowe. ${ }^{18}$ Thus, to make contact to these diodes, a sharp strong whisker is needed. The $25 \mu \mathrm{m}$ diameter Au/Ni alloy wire used as the antenna in this laser system is currently being etched with a $3 \% \mathrm{HCl}$ solution instead of the $10 \% \mathrm{KCN} / 5 \% \mathrm{Fe}(\mathrm{CN})_{6}$ solution used previously in the laboratory. A Pt wire and the whisker (dipped ca. $250 \mu \mathrm{m}$ into the solution) act as the electrodes for the electrochemical etching process. A DC voltage of $7 \mathrm{~V}$ is applied until enough metal has been etched away from the whisker so that it pops out of the liquid. This usually occurs after ca. $4-8$ seconds. At this point the whisker is usually perfect, however examination under the high power microscope should still be conducted to inspect the quality of the tip. Each whisker may be contacted several times on the 1T12 diode until it becomes too blunt, upon which it may then be used to 


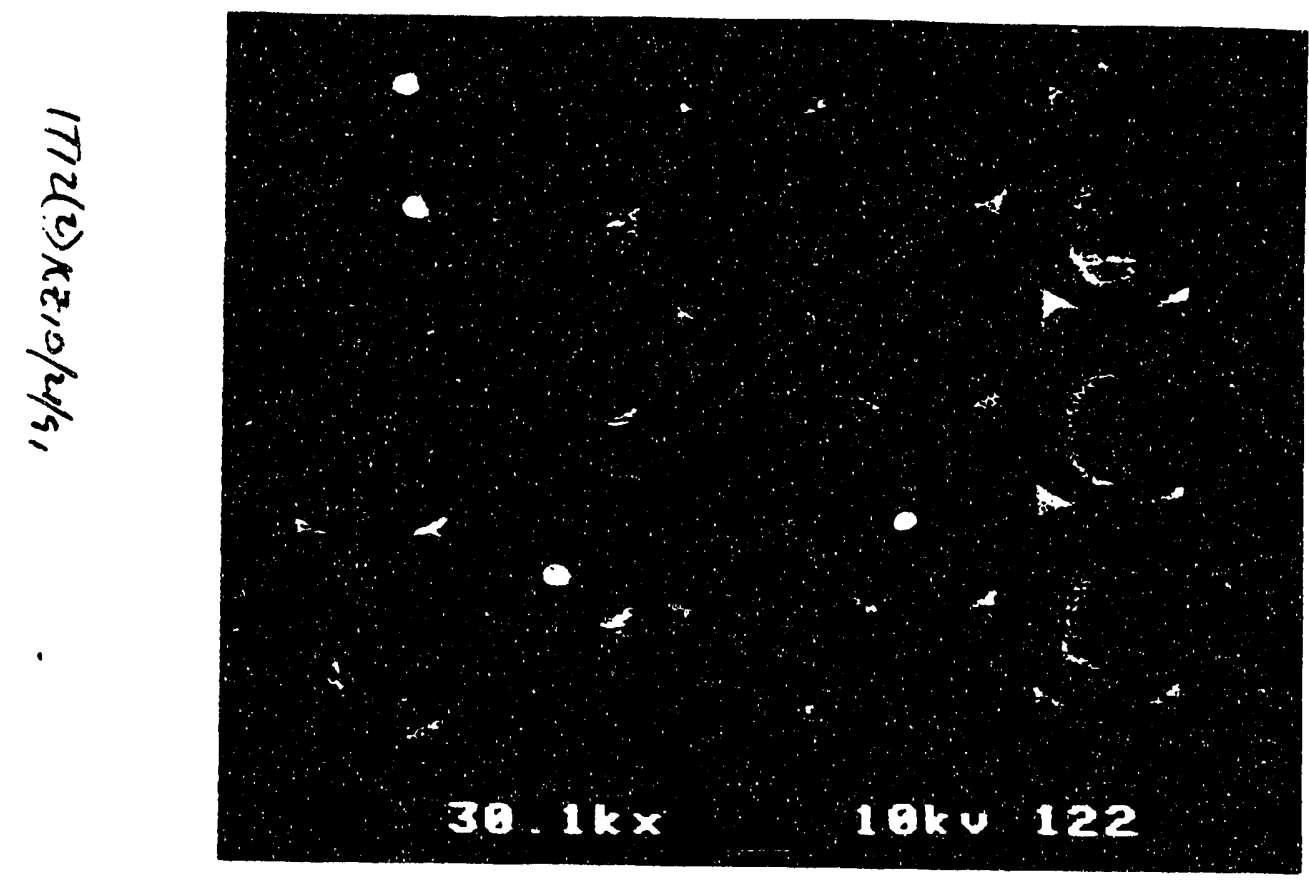

A Scanning Electron Micrograph of the 1T12 GaAs Schottky Barrier Diode.

contact a 117 diode. Also, transient voltage surges have been discussed in the section on the $\mathrm{CO}_{2}$ laser, and greater care must be taken when turning any piece of equipment on in the laboratory when the more sensitive diodes is installed. Regardless of the added difficulties in using these diodes it seems imperative that research continue along these lines to perfect the incorporation of these high quality chips into the tunable FIR laser system. The 1T13 is designed with the best specifications of any diode residing in the laboratory and should be attempted for scanning above $3 \mathrm{THz}$. Currently, the Crowe group at UVa have fabricated a series of diodes with an anode diameter of $0.25 \mu \mathrm{m}$, which exhibits a video response approximately 10 times greater than that of the 1 T12.18 Although this sounds like a formidable challenge, the bold experimenter would greatly benefit from such an enhanced sensitivity. 
In concluding this section it should be noted that for the water cluster experiments, more efficient sideband generation has been obtained in the spectral region above $2.4 \mathrm{THz}$ by utilizing the 1 T12 diode. Furthermore, by applying the formulae for optimal mixing efficiency as a function of whisker length derived by Zmuidzinas et al., 19 the design performance of the Martin-Puplett diplexer has been realized in the 2.5-3.0 THz spectral regime. These relationships are

$$
\begin{aligned}
\theta_{\max } & =\cos ^{-1}[1-0.37101 \lambda / \mathrm{L}] \\
\mathrm{s} & =0.971 / 2 \sin \theta_{\max },
\end{aligned}
$$

for which $\theta$ is the angle between the antenna (whisker) and the incident laser radiation, $\mathrm{L}$ is the whisker length, $\lambda$ is the wavelength of light, and $s$ is equal to the distance between the whisker and the corner cube reflector. By fixing $s$ and $\theta_{\max }$ at the values calculated from Equation 2.1, for a given $L$ and $\lambda$, the optimum performance of the diplexer was consistently maintained. In doing so, the optimal antenna mode of the whisker is coupled out of the comer cube mixer and this results in highly collimated double sideband radiation (full angle divergence of $50 \mathrm{mrad}$ at $2550 \mathrm{GHz}$ ) over the full length of the beam path. The added collimation helps in aligning the multipass cell so that the probe laser samples the cluster beam more effectively.

\subsection{Tunable Microwaves}

\subsubsection{General Microwave Technology; 2 - 20 GHz}

As discussed earlier in this chapter, laser coverage is very spotty above $3 \mathrm{THz}$. This prohibitive element of the spectrometer requires the improvement of the microwave tuning range, and this section is devoted to the description of the available equipment used for such a task. A Hewlett Packard (HP Model 8673B) microwave synthesizer supplies the fundamental frequency to the diode which is mixed with the fixed frequency carrier. By multiplying this radiation, the available coverage may be extended. The majority of this 
section will deal with the multiplication of the IF radiation. The HP synthesizer produces radiation from $2-26 \mathrm{GHz}$ and this can be easily coupled onto the diode via standard SMA (single mode transmission line from 1.1 - 18.0 GHz) coaxial cable. Using a Watkins Johnson Model A2000N (S/N 8F11) frequency doubler and an Hughes $8001 \mathrm{H}$ traveling wave tube amplifier, the IF may be doubled up to $40 \mathrm{GHz}$. The test data for the $\mathrm{A} 2000 \mathrm{~N}$ is given in Table 2.3. IF radiation from $2-40 \mathrm{GHz}$ has been commonly used for several years to tune sideband radiation off of many closely spaced FIR lasers. However, as the laser coverage becomes more sparse, it becomes clear that the tuning range of the IF needs to be extended.

\subsubsection{0 - 65 GHz Tuning of the FIR Laser}

An HP model 8349B 2 - $20 \mathrm{GHz}$ solid state amplifier and a Watkins Johnson Model Q2300N (S/N 8F10) frequency tripler are available for obtaining coverage up to 60 $\mathrm{GHz}$. The test data for the $\mathrm{Q} 2300 \mathrm{~N}$ are also shown in Table 2.3. Figure 2.6 shows the microwave rectification between $39-60 \mathrm{GHz}$ when using standard SMA coaxial connections with the Q2300N tripler. The curve represented by the solid circles was taken with an SMA bias tee, whereas a $\mathrm{K}$ - type $\mathrm{DC}-40 \mathrm{GHz}$ bias tee was used to obtain the curve represented by the open squares. It is easily noticed that the installation of the $\mathrm{K}$ bias tee provided an improvement in the amount of microwave rectification on the diode, yet it is still far below an optimum level (at least $100-200 \mathrm{mV}$ ) needed to generate a sufficient amount of sidebands which may be used for scanning.

To improve the transmission of the IF onto the Schottky diode, the entire corner cube has been converted over to $\mathrm{V}-\mathrm{type}, \mathrm{DC}-65 \mathrm{GHz}$ connectors. In Figure 2.7 two power curves as a function of frequency are shown, to illustrate the higher quality of microwave transmission onto the diode with the $\mathrm{V}$ - connectors. The variation of the two curves is most probably caused by slight imperfections in the grounding scheme, which may introduce unwanted mode characteristics due to impedance mismatching of the 
Table 2.3

Test data for Watkins and Johnson Frequency Doubler and Tripler show the output frequencies and the power output. The input for the doubler and tripler was fixed at 25 and $20 \mathrm{~mW}$, respectively. Maximum input powers for the doubler and tripler should not exceed $100 \mathrm{~mW}(20 \mathrm{dBm})$ and $63 \mathrm{~mW}(18 \mathrm{dBm})$, respectively.

\begin{tabular}{ll|l}
\multicolumn{3}{c}{ A2000N Doubler } \\
\hline $26.6 \mathrm{GHz}$ & $2.7 \mathrm{~mW}$ \\
28 & $\mathrm{GHz}$ & $3.2 \mathrm{~mW}$ \\
29 & $\mathrm{GHz}$ & $3.2 \mathrm{~mW}$ \\
30 & $\mathrm{GHz}$ & $3.4 \mathrm{~mW}$ \\
31 & $\mathrm{GHz}$ & $3.8 \mathrm{~mW}$ \\
32 & $\mathrm{GHz}$ & $3.5 \mathrm{~mW}$ \\
33 & $\mathrm{GHz}$ & $3.3 \mathrm{~mW}$ \\
34 & $\mathrm{GHz}$ & $4.7 \mathrm{~mW}$ \\
36 & $\mathrm{GHz}$ & $4.5 \mathrm{~mW}$ \\
37 & $\mathrm{GHz}$ & $3.6 \mathrm{~mW}$ \\
38 & $\mathrm{GHz}$ & $3.4 \mathrm{~mW}$ \\
39 & $\mathrm{GHz}$ & $3.5 \mathrm{~mW}$ \\
40 & $\mathrm{GHz}$ & $2.5 \mathrm{~mW}$ \\
\hline
\end{tabular}

\begin{tabular}{ll|ll}
\multicolumn{3}{c}{ Q2300N Tripler } \\
\hline 40 & $\mathrm{GHz}$ & $.90 \mathrm{~mW}$ \\
41 & $\mathrm{GHz}$ & $.92 \mathrm{~mW}$ \\
42 & $\mathrm{GHz}$ & $.77 \mathrm{~mW}$ \\
44 & $\mathrm{GHz}$ & $.77 \mathrm{~mW}$ \\
46 & $\mathrm{GHz}$ & $.64 \mathrm{~mW}$ \\
48 & $\mathrm{GHz}$ & $.67 \mathrm{~mW}$ \\
50 & $\mathrm{GHz}$ & $1.00 \mathrm{~mW}$ \\
52 & $\mathrm{GHz}$ & $.93 \mathrm{~mW}$ \\
54 & $\mathrm{GHz}$ & $1.07 \mathrm{~mW}$ \\
56 & $\mathrm{GHz}$ & $1.23 \mathrm{~mW}$ \\
58 & $\mathrm{GHz}$ & $1.36 \mathrm{~mW}$ \\
59 & $\mathrm{GHz}$ & $.98 \mathrm{~mW}$ \\
60 & $\mathrm{GHz}$ & $.75 \mathrm{~mW}$ \\
\hline
\end{tabular}


Figure 2.6

Tripled Microwave Power on Diode vs. Frequency $(17.2 \mathrm{dBm})$

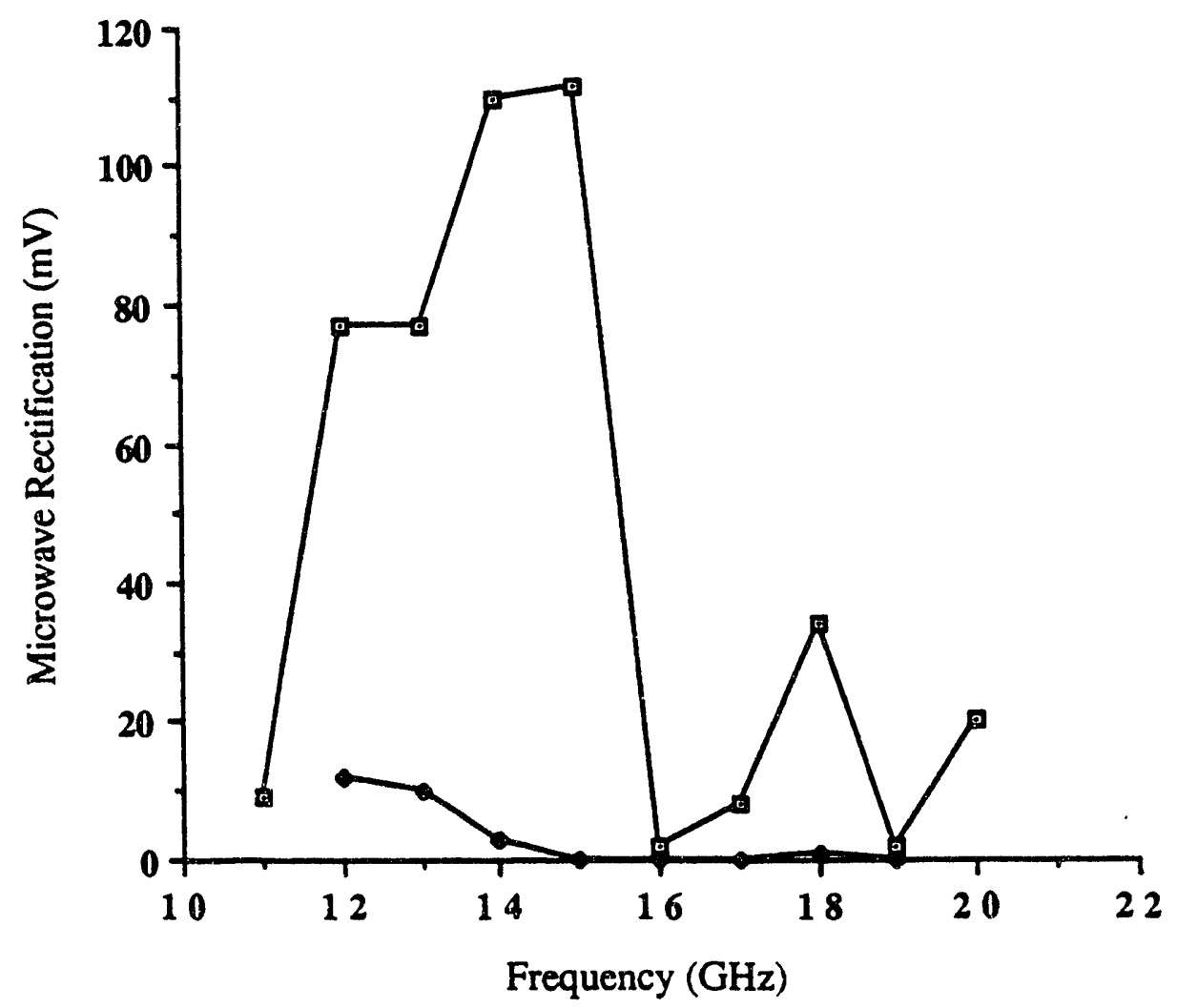

This figure demonstrates the transmission characteristics of $\mathrm{K}$ type cables which are specified as single mode transmission line from DC to $40 \mathrm{GHz}$. The curve represented with the black diamonds was measured with an SMA bias tee, whereas the data corresponding to the open squares was measured with a $\mathrm{K}$ type bias tee. This comparison indicates that the cabling scheme is inadequate for the propagation of microwaves in the 40 - $60 \mathrm{GHz}$ frequency range. 
Figure 2.7

Tripled Microwave Power on Diode vs. Frequency $(18.0 \mathrm{dBm})$

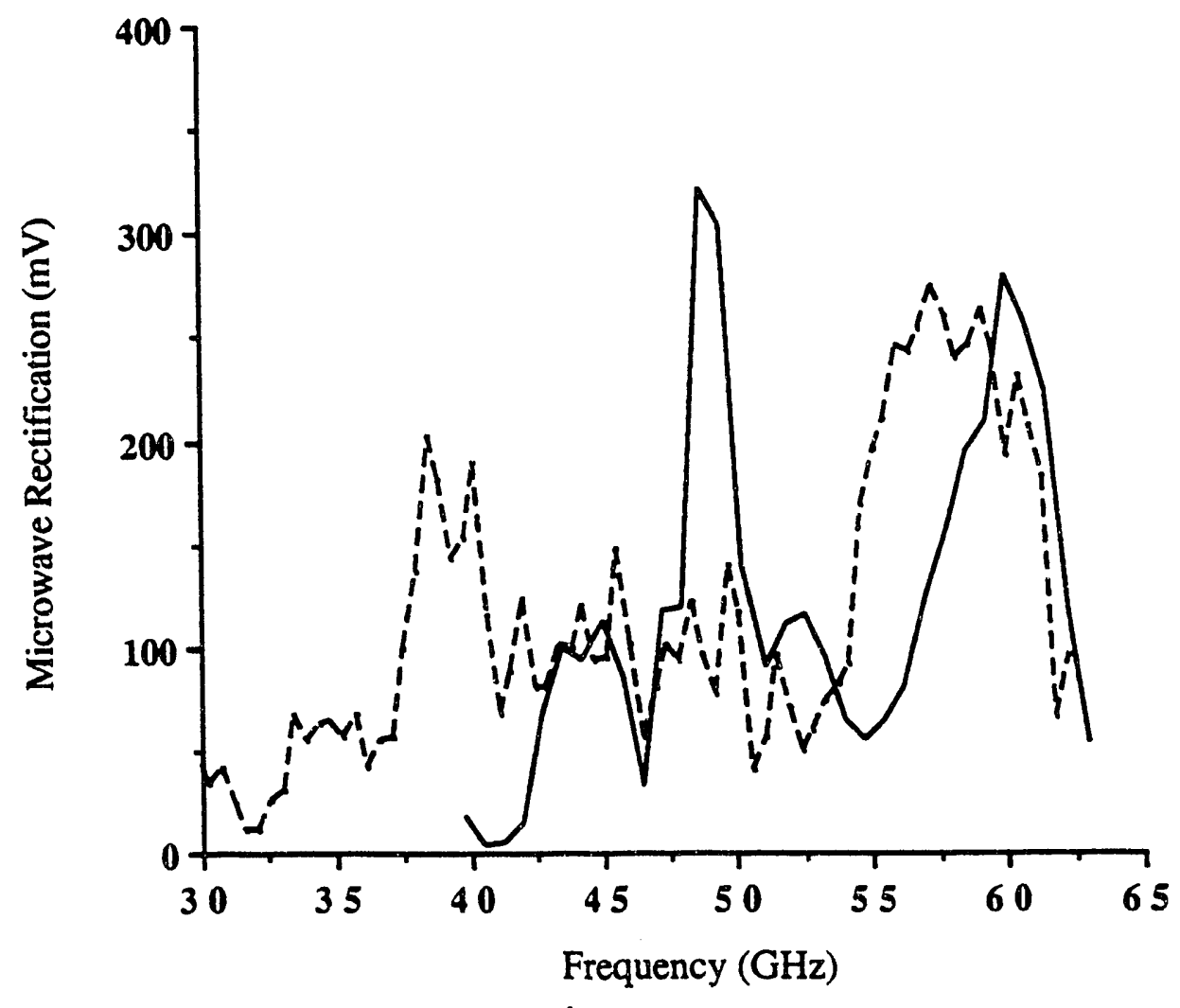

These two curves are both taken with the V transmission cable. It is readily noticed that the microwave coverage can be adequately extended using the Watkins Johnson Q2300 tripler. Although the diode rectification is less than the $400 \mathrm{mV}$ that is usually applied to the mixer, it is sufficient. The difference of the two data sets most likely comes from the fact that two they were measured with different whiskers. It was found that a good electrical contact was necessary for optimum performance of the transmission line. This was not the case for the solid line. 
radiation isnto the diode. It is therefore important to be sure that the contact of each connection is made properly and tightly. One way to remove a source of error in the cabling would be to solder each $\mathrm{V}$ - bead into its own flange launcher insert which would also help in the handling of the smaller bead size, thus preventing whisker mutilation.

Although the microwave rectification is sufficient for scanning between $40-63$ $\mathrm{GHz}$, more power is needed to maintain the highest level of sensitivity, because the tripler does not perform nearly as well as the doubler, for example, which yields more than enough power to saturate the diode. To overcome this power limitation, an Avantek SMW91-2920 40-60 GHz amplifier has been purchased as an engineering prototype to provide sufficient power in the $40-60 \mathrm{GHz}$ frequency range. The quoted specifications for this unit are shown in Table 2.4.

Table 2.4

Specifications for the 40 - 60 Avantek Amplifier

\begin{tabular}{l|l}
\hline \hline Frequency Range & $40-60 \mathrm{GHz}$ \\
Gain & $20 \mathrm{~dB}$ \\
Gain Flamess & $+/-3 \mathrm{~dB}$ \\
Noise Figure & $8.5 \mathrm{~dB}$ \\
VSWR in/out & $3: 1$ \\
\hline
\end{tabular}

\begin{tabular}{l|l}
\hline Input Voltage & $12 \mathrm{~V} \mathrm{DC}$ \\
Input Current & $250 \mathrm{~mA} / 350 \mathrm{~mA} \max$ \\
Case Temp Range & $25^{\circ} \mathrm{C}$ \\
Connectors & $\mathrm{V}-$ type \\
Case & $\mathrm{T} 4 \mathrm{~V}$ \\
\hline
\end{tabular}

\subsection{2 $65 \cdot 110 \mathrm{GHz}$ Tuning of the FIR Laser}

Tuning capabilities exist for the frequency range from $65-110 \mathrm{Ghz}$ through the use of an InP Gunn diode (Model EE 893-038) obtained from J. Carlstrom. The Gunn Diode is housed in a compact container (Model Number H116) upon which two precision micrometers and an SMA jack are mounted. High frequency microwave radiation travels through WR-10 waveguide which can be attached to the exit port of the H116 and 
connected to the similar waveguide mounted on the corner cube mixer. Absolute power output versus frequency for this diode is plotted in Figure 2.8 across its entire tuning range. This data was collected with an HP432A power meter and a Hughes $45776 \mathrm{H}-1100$ microwave detector. The frequency was measured by splitting ca. $10 \%$ of the microwaves out of the waveguide with a directional coupler (Aerowave 10-4040/10), and sending this radiation to a precise frequency counter. Before entering the detector, the radiation was attenuated with a Hughes $45726 \mathrm{H}-1000$ variable attenuator.

\section{Figure 2.8}

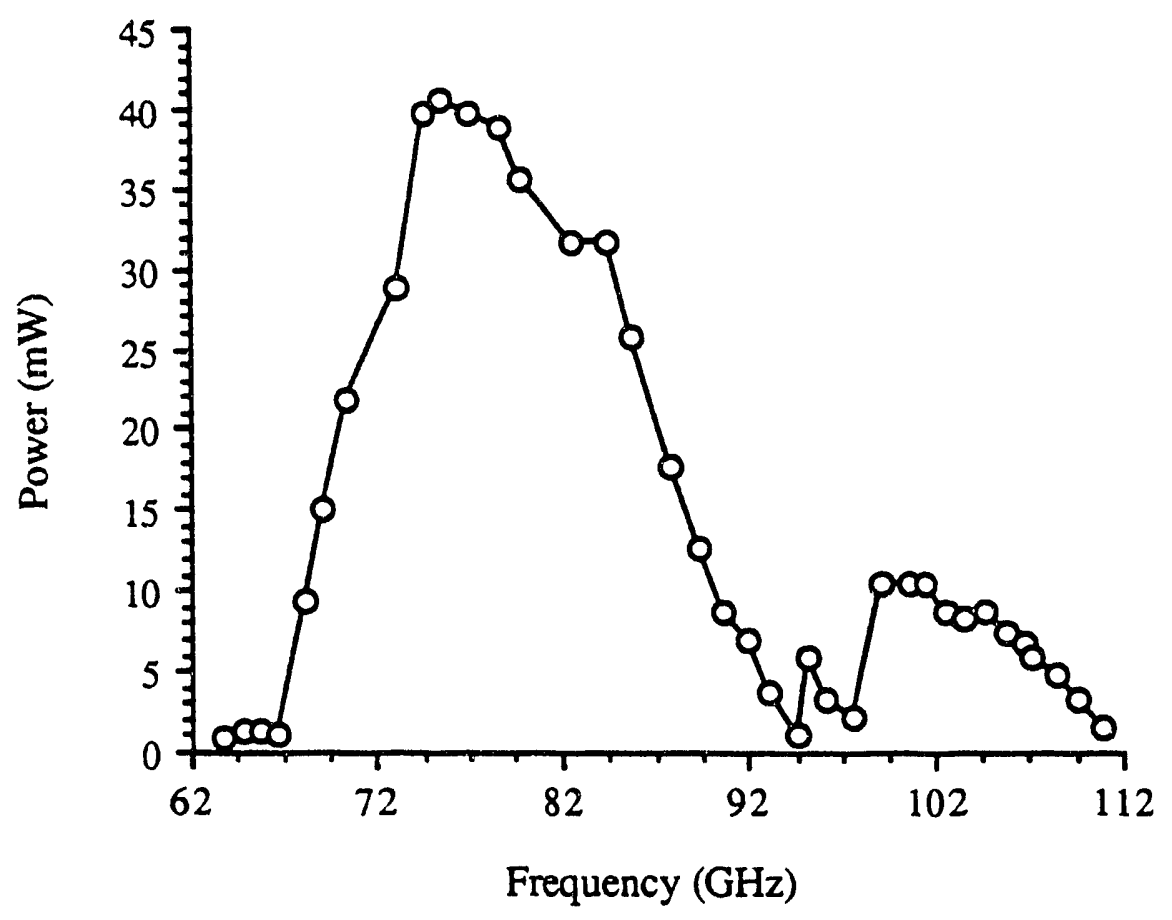

The power output of the EE 893-038 Gunn diode with respect to frequency.

A diagram of a proposed method for operating the Gunn diode with the open structure corner cube receiver is shown in Figure 2.9. During the operation of the Gunn oscillator, the HP 8349B synthesizer should be set at a frequency which will supply the desired frequency through an appropriate harmonic. For example, if a particular absorption 
Figure 2.9

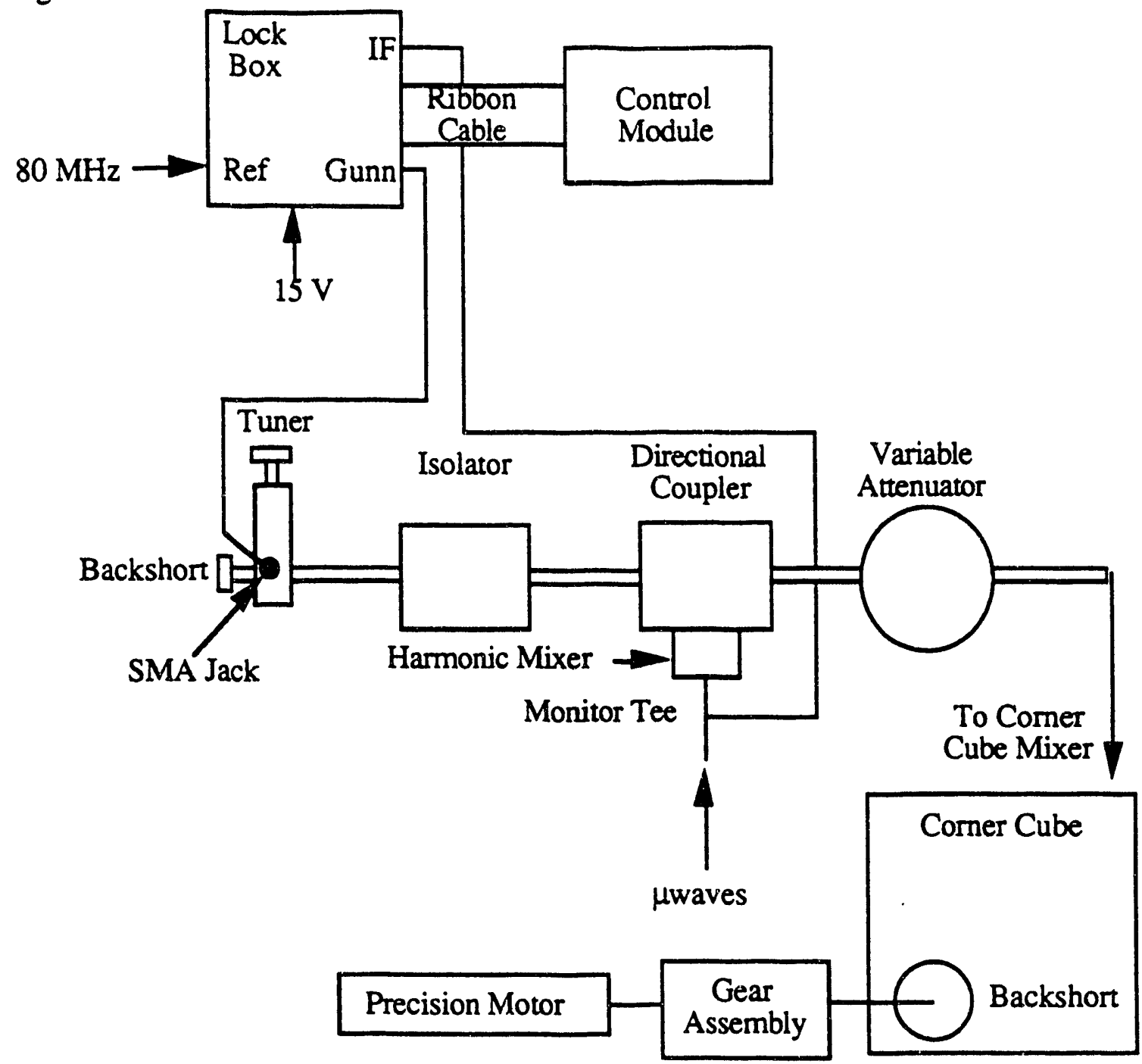

A block diagram showing the proposed setup for operating the Gunn diodes with the Berkeley FIR laser absorption spectrometer.

feature has a frequency of $v_{\text {absorption }}=v_{\text {laser }}+73681.02 \mathrm{MHz}$, the HP could be set up to Absolute power versus frequency of the EE 893-038 Gunn diode.generate the 10th harmonic at $7368.102 \mathrm{MHz}$. However, it is important to remember that the Gunn diode always oscillates at a frequency which is $80 \mathrm{MHz}$ higher than the nth harmonic of the HP, because of the $80 \mathrm{MHz}$ reference supplied to the lock box. With this in mind the microwave synthesizer should be set up at $(73681.02-80.0) / 10=7360.102 \mathrm{MHz}$ to obtain the desired frequency of $73681.02 \mathrm{MHz}$. To lock the Gunn at a given frequency, 
Table 2.5

The tuning curve as measured by G. A Blake for the EE 893-038 Gunn diode. Several backshort positions may maximize the out ut coupling efficiency for a particular frequency, yet the values listed for the tuner are $\mathrm{I}$ producible as was determined during the power measurement experiment reported in Figure 2.8. The notes in the last column indicate the amount of microwave rectification onto a 117 diode mounted in the CalTech corner cube mixer. The quotation " can swing diode negative " indicates that there is $>750 \mathrm{mV}$ of rectification at that frequency.

\begin{tabular}{llll} 
Frequency $(\mathrm{GHz})$ & Tuner & Backshort & G. A. Blake's Notes \\
\hline 67.08 & 92.9 & 165 & can swing diode negative \\
68.08 & 89.0 & 181 & can swing diode negative \\
69.08 & 79.0 & 176 & can swing diode negative \\
70.08 & 71.8 & 164 & can swing diode negative \\
71.08 & 66.5 & 157 & can swing diode negative \\
72.08 & 61.6 & 112 & can swing diode negative \\
73.08 & 57.3 & 143 & can swing diode negative \\
74.08 & 53.2 & 153 & can swing diode negative \\
75.08 & 49.5 & 150 & can swing diode negative \\
76.08 & 47.1 & 183 & can swing diode negative \\
77.08 & 43.9 & 124 & $\max =490 \mathrm{mV}$ \\
78.08 & 41.2 & 124 & $\max =470 \mathrm{mV}$ \\
79.08 & 38.2 & 101 & $\max =580 \mathrm{mV}$ \\
80.08 & 36.0 & 75 & $\max =560 \mathrm{mV}$ \\
81.08 & 33.6 & 70 & $\max =90 \mathrm{mV}$ \\
82.08 & 26.5 & 55 & $\max =430 \mathrm{mV}$ \\
83.08 & 24.7 & 36 & can swing diode negative \\
84.08 & 22.9 & 28 & can swing diode negative \\
85.08 & 26.2 & 106 & can swing diode negative \\
86.08 & 24.6 & 98 & can swing diode negative \\
87.08 & 23.1 & 76 & can swing diode negative \\
88.08 & 21.8 & 71 & can swing diode negative \\
89.08 & 20.7 & 65 & $\max =420 \mathrm{mV}$ \\
90.08 & 19.4 & 60 & can swing diode negative \\
91.08 & 18.4 & 66 & $\max =100 \mathrm{mV}$ \\
92.08 & 17.4 & 58 & No Coupling Detected \\
93.08 & 16.3 & 49 & $\max =30 \mathrm{mV}$ \\
94.08 & 15.5 & 53 & $\max =20 \mathrm{mV}$ \\
95.08 & 14.7 & 82 & $\max =90 \mathrm{mV}$ \\
96.08 & 14.0 & 67 & $\max =10 \mathrm{mV}$ \\
97.08 & 13.1 & 50 & $\max =5 \mathrm{mV}$ \\
98.08 & 12.3 & 71 & $\max =5 \mathrm{mV}$ \\
99.08 & 11.8 & 62 & $\max =80 \mathrm{mV}$ \\
100.08 & 11.1 & 49 & $\max =25 \mathrm{mV}$ \\
101.08 & 10.4 & 43 & $\max =100 \mathrm{mV}$ \\
102.08 & 9.8 & 39 & $\max =25 \mathrm{mV}$ \\
103.08 & 9.2 & 35 & $\max =20 \mathrm{mV}$ \\
104.08 & 8.8 & 32 & $\max =30 \mathrm{mV}$ \\
105.08 & 8.2 & 29 & $\max =10 \mathrm{mV}$ \\
106.08 & 7.7 & 27 & $\max =5 \mathrm{mV}$ \\
107.08 & 24 & $\max =15 \mathrm{mV}$ \\
108.08 & 21 & $\max =6 \mathrm{mV}$ \\
& & &
\end{tabular}


the backshort and the Gunn tuner, should be precisely set at the positions listed in Table 2.5, which was measured by Professor G. A. Blake, and J. Carlstrom ${ }^{20}$ with the corner cube located on the CalTech tunable FIR laser system. Once locked, it is important to change the microwave input setting to a different harmonic (ie. the 9th or 11 th for the example shown above) to make certain that the lock box is set up properly. When comparing the measured power output curve of Figure 2.8 with the CalTech data listed in Table 2.5, it is noticed that the observed roll off of transmission of the microwaves onto the corner cube antenna corresponds to a decrease in power output of the Gunn diode. Thus, designing a corner cube mixer which acts as a good coupling device above $95 \mathrm{GHz}$ seems unnecessary until sufficient microwave power can be obtained in this frequency range. ${ }^{21}$ In conclusion, with the addition of the V-type cabling and the Gunn diodes to the previously available microwave components, sufficient power is now available to tune any FIR laser +/- 2-110 GHz.

\subsection{Detectors; Ge:Ga Photoconductors}

To detect radiation above in the frequency range of the FIR $>2.0 \mathrm{THz}, \mathrm{Ge}: \mathrm{Ga}$ is the material of choice. ${ }^{22}$ This semiconductor operates as a photoconductive detector which is responsive to radiation in the frequency range from ca. $50-240 \mathrm{~cm}^{-1}$. In Figure 2.10 , the relative responsivity of the currently available photoconductor, is plotted versus frequency. For this detector the FWHM spans a region of the FIR between $80-200 \mathrm{~cm}^{-1}$. The conditions of the scan are described in the Figure caption. When applying uniaxial mechanical stress along the (100) axis of the crystal, the FWHM of the wavelength range is shifted to as low as $50 \mathrm{~cm}^{-1} .23$ Such a detector will be referred to as a stressed photoconductor (SPC), whereas the normal version for which the responsivity curve is shown in Figure 2.10, will be referred to as the unstressed photoconductor (UPC). There are two versions of the SPC, and they are distinguished from one another by the labels old and new (O or N, respectively )SPC. The main difference between the two is that the the 
Figure: 2.10

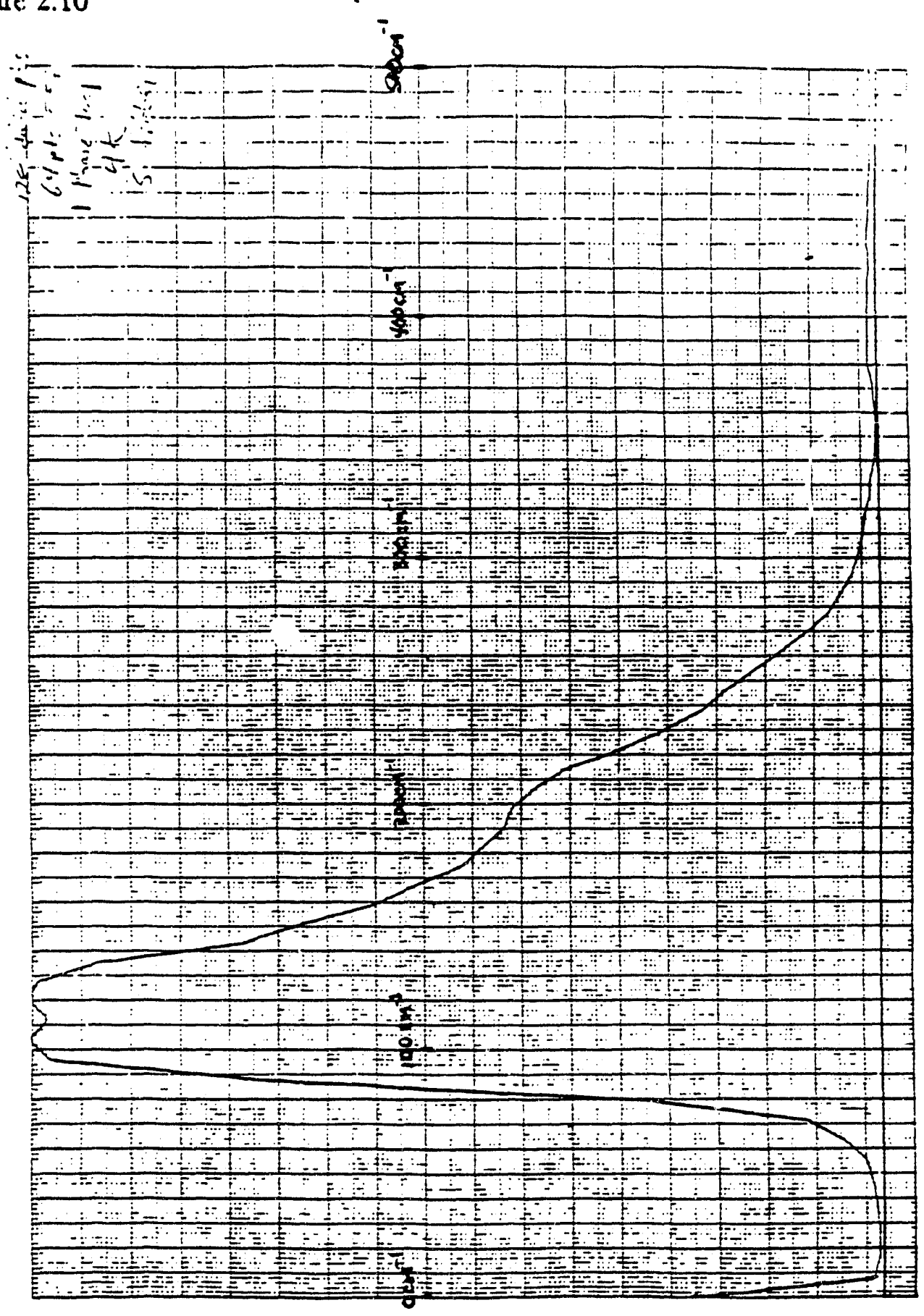

A relative responsivity for the UPC. This data was recorded at a detector temperature of $4.2 \mathrm{~K}$. Cooling the detector down to $2.7 \mathrm{~K}$ produced no observable change in responsivity. 
OSPC has a fixed detector bias voltage of $250 \mathrm{mV}$ and a low noise (warm) preamplifier which is operational for detectors possessing impedance values of $\geq 1 \mathrm{k} \Omega, 24$ whereas both the NSPC and UPC have variable detector bias circuitry associated an Infrared Labs LN8 Preamp. Each detector has a set of cold ind warm resistances which may be considered as test data for the individual units. This data are shown in Table 2.5.

Table 2.5

The resistances of each $\mathrm{Ge}: \mathrm{Ga}$ photoconductor and the feedback resistors for each. The $4 \mathrm{~K} /$ dark data corresponas to a $4 \mathrm{~K}$ detector viewing a $4 \mathrm{~K}$ blackbody, and the $4 \mathrm{~K} / 300 \mathrm{~K}$ BB indicates that the detector if viewing room temperature radiation through a garnet powder filter.

\begin{tabular}{l|lll}
\hline & OSPC & $N S P C$ & $U P C$ \\
\hline $\mathrm{R}_{4 \mathrm{~K} / \mathrm{dark}}$ & $\ldots$ & $460 \mathrm{k} \Omega$ & $5.5 \mathrm{M} \Omega$ \\
$\mathrm{R}_{4 \mathrm{~K} / 300 \mathrm{~K} B B}$ & $27 \mathrm{k} \Omega$ & $78.3 \mathrm{k} \Omega$ & $191 \mathrm{k} \Omega$ \\
$\mathrm{R}_{\mathrm{RM} T E M P}$ & $367 \Omega$ & $944 \Omega$ & $114 \Omega$ \\
$\mathrm{R}_{\text {FEEDBACK }}$ & $99.4 \mathrm{k} \Omega$ & $47.7 \mathrm{k} \Omega$ & $901 \mathrm{k} \Omega$ \\
\hline
\end{tabular}

From this data it is clear that although the $4 \mathrm{~K} /$ dark data varies considerably among the detectors, the operational data $(4 \mathrm{~K} / 300 \mathrm{~K} \mathrm{BB})$ enly varies by at most a factor of seven It would appear that identical preamps would be sufficient for all three units.

Current vs. Bias Voltage (IV) curves for the UFC are shown in Figure 2.11 for the detector viewing varying amounts of radiation. The $4 \mathrm{~K} /$ dark IV data is essentially the detector noise versus bias voltage over the entire noise spectrum. If the incident power on the detector is assumed to be ca. $10 \mu \mathrm{W}$ (assuming a conversion efficiency of ca. $0.0001^{5,25}$ ), a value for the detector responsivity $(\mathcal{R})$ can be estimated to be $0.2 \mathrm{~A} / \mathrm{W}$ at a bias of $0.6 \mathrm{~V}$. This value seems a bit low, k:owever the detector may exhibit nonlinear values for $\mathcal{R}$ when imaging the relatively high power sidebands onto the element. 
Figure 2.11

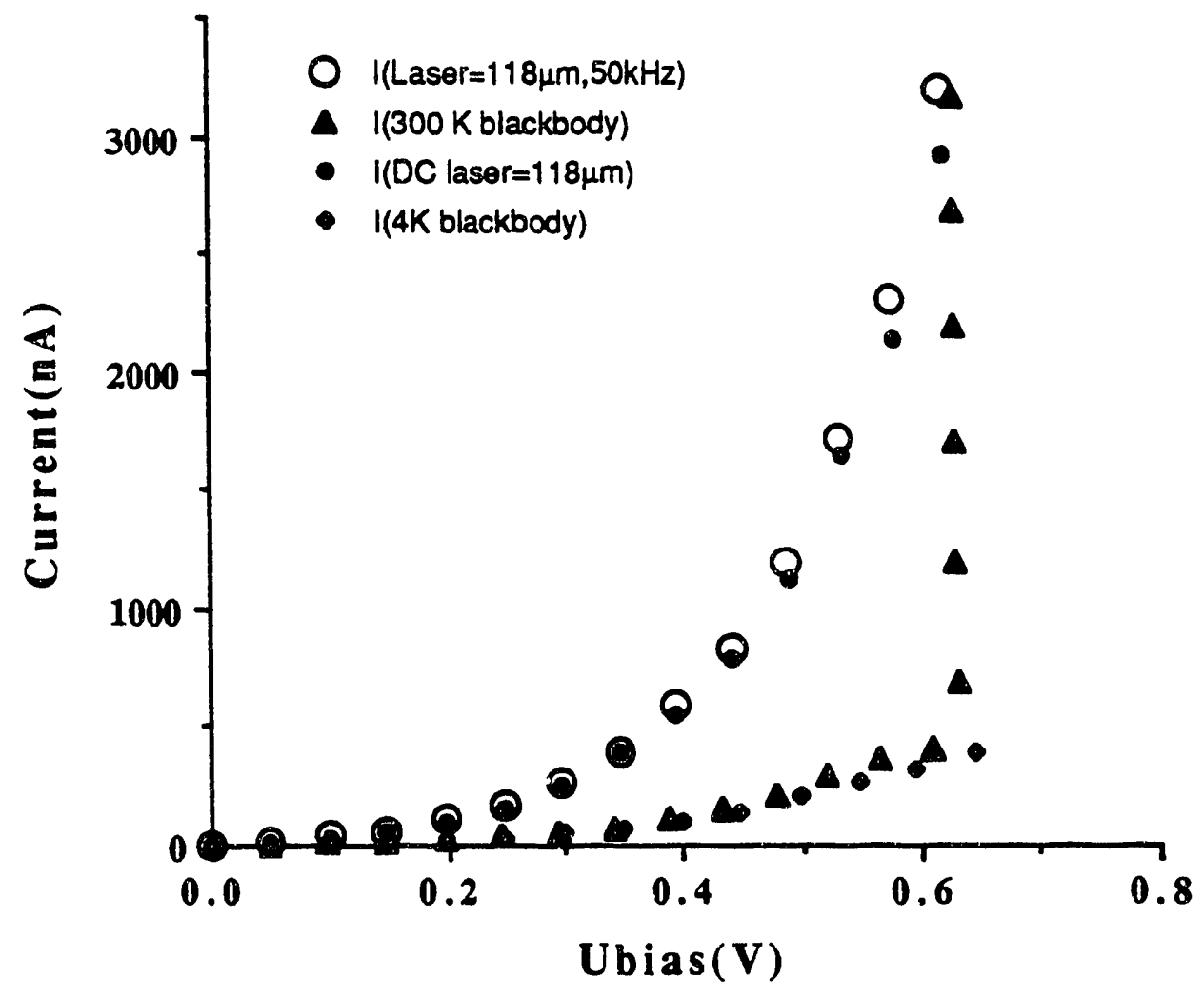

Four IV curves for the UPC are plotted for the conditions listed in the legend. These data were measured as current directly out of the detector with a picoammeter, and no preamplification circuitry was employed. The signal to noise at the $100 \mathrm{kHz}$ detection frequency can be measured in the Haller laboratory located in Cory Hall. A lockin amplifier with a picoammeter plugin may be used to obtain the true $S / N$ ratio of only the detector.

By measuring the $\mathrm{S} / \mathrm{N}$ of the pure detector, a maximum level of sensitivity may be defined. These IV curves, measured at the detection frequency of $100 \mathrm{kHz}$, and an absolute power measurement of the sideband radiation, will define the true performance specification of the UPC element. The data will allow for the characterization of the product of the photoconductive gain $\left(G_{p c}\right)$ and the quantum efficiency $(\eta)$ for the detector through the following, 


$$
I=F \text { e } \eta G_{p c}
$$

for which $e$ is the charge of a carrier, and $F$ is the number of photons per second on the detector. The product of $\eta G_{p c}$ should be as large as possible, and would typically be in the range of $0.01-0.1$.

The highest sensitivity of $1.3 \times 10^{6}$ for the UPC has been measured as the ratio of double sideband power to the noise at a modulation frequency of $20 \mathrm{kHz}$ ( $1 \mathrm{~Hz}$ bandwidth) and at $\lambda=118 \mu \mathrm{m}$. Unfortunately, the detector currently operates under preamp noise limited conditions at all modulation frequencies. This situation becomes prohibitive near the detection frequency of $100 \mathrm{kHz}$. Better preamps are under consideration which correct this problem as well as match the impedance characteristics of the element (which is nominally $10-100 \mathrm{k} \Omega$ at ca. $200-400 \mathrm{mV}$ of bias, depending on the detector) more efficiently.

\subsection{Conclusion}

In conclusion, the coverage of the FIR laser absorption spectrometer may be extended toward the 4 - $5 \mathrm{THz}$ spectral range by continuing efforts related to detection, microwave coverage, and diode technology. Part of these developments have been employed to obtain the present data, however a great deal of work still remains. The majority of the equipment necessary for this purpose is currently in the laboratory, and only the installation of each piece of technology is required to further advance the spectrometer performance. Through the extension of the spectral coverage, the capabilities of the FIR laser absorption spectrometer will be enhanced, for high resolution tunable radiation sources at these frequencies will find a wide range of applications. In particular, the study of intermolecular vibrations of water clusters will become readily accessible. Although initial efforts regarding such experiments are discussed in the following chapters, the 
possibilities for the complete characterization of water from small clusters to the bulk may be accomplished as the developments of the spectrometer are pursued. 
1 G. A. Blake, K. B. Laughlin, R. C. Cohen, K. L. Busarow, D-H. Gwo, C. A. Schmuttenmaer, D. W. Steyert, and R. J. Saykally, Rev. Sci. Instrum. 62, 1693 (1991).

2 G. A. Blake, K. B. Laughlin, R. C. Cohen, K. L. Busarow, D-H. Gwo, C. A. Schmuttenmaer, D. W. Steyert, and R. J. Saykally, Rev. Sci. Instrum. 62, 1701 (1991).

3 C. A. Schmuttenmaer, Thesis, University of California (1991).

4 D. W. Steyert, Thesis, University of California (1991).

5 R. C. Cohen, Thesis, University of California (1991).

6 K. L. Busarow, Thesis, University of California (1991).

7 K. B. Laughlin, Thesis, University of California (1989).

8 A. E. Siegman, Lasers (University Science Books, Mill Valley Ca. 1986).

9 The autocollimating site can be borrowed from the Y. T. Lee laboratory and its usage will perfect the alignment of the $\mathrm{CO} 2$ laser tubes.

10 J. Farhoomand, G. A. Blake, M. A. Frerking, and H. M. Pickett, J. Appl. Phys. 57, 1763 (1985).

11 J. J. Degnan, Appl. Phys. 11, 1 (1976).

12 R. L. Abrams and A. N. Chester, Appl. Optics 13, 2117 (1974).

13 T. W. Crowe, Intern. J. Infrared and Millimeter Waves, 10, 765 (1989).

14 For examples of other types of heterodyne recievers used with FIR radiation, see J. O. Marsh, T. W. Crowe, and J. Hesler, Intern. J. Infrared and Millimeter Waves, 11, 1113 (1990).; W. L. Bishop, T. W. Crowe, R. J. Mattauch, and P. H. Ostdiek, Microwave and Opical Technology Letters, 4, 44 (1991).

15 H. Krautle, E. Sauter, and G. V. Schultz, Infrared Physics, 17, 477 (1977). 
16 For the interested reader see; W. C. B. Peatman and T. W. Crowe, Intern. J. Infrared and Millimeter Waves, 11, 355 (1990).

17 R. U. Titz, H. P. Roser, G. W. Schwaab, H. J. Neilson, and others, Intern. J. Infrared and Millimeter Waves, 11, 809 (1990).

18 Professor T. W. Crowe, private communication.

19 J. Zmuidzinas, A. L. Betz and R. T. Borenko, Infared Physics 29, 119 (1989).

20 G. A. Blake and J. Carlstrom, Private Communication.

21 Currently Carlstrom Gunn diodes are operational up to $150 \mathrm{GHz}$ with ca. $30 \mathrm{~mW}$ of power - G. A. Blake, Private Communication.

22 P. R. Bratt, Semiconductors and Semimetals; Infrared Detectors 12, 39 (Academic Press, New York, NY 1977).

23 E. E. Haller, Infrared Physics, 25, 257 (1985).

24 E. R. Brown, Electronics Lett., 21, 417 (1985).

25 H. Nett and B. J. Clifton, Intern. J. Infrared and Millimeter Waves, 11, 1333 (1990). 


\section{Chapter 3}

\section{Vibrational and $\mathbf{K}_{\mathrm{a}}{ }^{\prime}$ Dependencies of the Multidimensional Tunneling Dynamics in the $82.6 \mathrm{~cm}^{-1}$ Intermolecular Vibration of the Water Dimer-d4}

\subsection{Introduction}

The facile quantum tunneling dynamics of hydrogen atoms in the water dimer have been examined with high resolution spectroscopic techniques for nearly two decades, 1 following the pioneering study of Dyke, Mack and Muenter. ${ }^{2}$ The vibration-rotationtunneling (VRT) energy levels are currently understood in terms of permutation-inversion (PI) group theory, ${ }^{3}$ and by considering each of the tunneling coordinates as an isolated, adiabatic motion via the generalized internal-axis-method (IAM) developed by Coudert and Hougen. 4,5 As a result, this cluster has become an important prototype in the treatment of multidimensional tunneling dynamics in clusters. In the current VRT models, the barriers on the multidimensional intermolecular potential energy surface (IPS) through which the tunneling occurs are assumed to be much larger than the VRT splittings induced by the motions. Measurement of the intermolecular vibrations will provide a critical test of the applicability of this approximation because these motions sample regions of the IPS that are approximately isoenergetic with the tunneling barrier maxima. Until recently, however, such measurements have not been technologically feasible.

Throughout the past several years, the ground vibrational states for all possible water dimer isotopomers have been examined in some detail with high resolution microwave and far infrared (FIR) spectroscopic probes. $1,6,7,8$ In particular, the $\mathrm{K}_{\mathrm{a}}{ }^{\prime \prime}=0$, 
1 , and 2 manifolds of the normal and fully deuterated species have been extensively studied, and these data have provided a relatively complete characterization of the ground state VRT energy levels of these clusters in terms of Hougen's generalized IAM model. ${ }^{9}$ Recently, the first high resolution spectrum of an excited intermolecular vibration ${ }^{10}$ has been located near $82.6 \mathrm{~cm}^{-1}$ and the five VRT subbands observed were rationalized in terms of ab initio predictions, the measured a- and c-type VRT spectra, and the fitted tunneling splittings. Since that work, seven additional VRT subbands of this vibration have been detected, allowing for the complete characterization of the excited state $\mathrm{K}_{\mathrm{a}}=0$ and 1 manifolds. The resulting excited state energy level diagram verifies that all of the tunneling motions posses extremely different characteristics from those in the ground state, as evidenced by the measured VRT splittings. The high barrier approximation assumed for the treatment of the ground state of the water dimer defines the barrier through which a tunneling motion passes to be much larger than the associated splitting, and this is the basis for treating each tunneling motion as an independent coordinate. The data presented in this work lead us to question the validity of this approximation for describing the intermolecular vibrational degrees of freedom. Finally, the measurement of the intermolecular vibrations are also a necessary step for the ultimate determination of an accurate intermolecular pair potential describing the water-water interaction, and FIR-VRT laser absorption spectroscopy has evolved as a highly sensitive probe of these motions. ${ }^{11}$

The data and subsequent discussions within this chapter are organized in the following way. In Section 3.2, a brief description of the specific operating conditions used to collect this data are discussed. In Section 3.3, the details regarding the group theoretical treatment of the water dimer are presented, as are the assignment of the six $K_{a}=0>0$ and six $\mathrm{K}_{\mathrm{a}}=0 \rightarrow 1$ subbands. Section 3.4 contains an examination of the strongly coupled excited state tunneling splittings which are compared to the current understanding of quantum tunneling in the vibrational ground state. The $\mathrm{K}_{\mathrm{a}}{ }^{\prime}$ dependencies are particularly interesting, and relevant examples of related phenomena occurring in other molecular 
systems are described for comparative purposes. In the concluding discussion of Section 3.5, the intermolecular vibrational coordinate be considered within the present excited state tunneling picture. An isotopically substituted intermolecular vibrational band for the $\mathrm{D}_{2} \mathrm{O}$ HOD species is also presented in this section, because these data are consistent with the present vibrational assignment. Finally, preliminary considerations for calculating the dynamics on an IPS will be discussed.

\subsection{Water Dimer Experimental Conditions}

Only a brief description of the specific experimental configuration will be presented below for the water dimer experiments. The frequencies of the FIR lasers ${ }^{12}$ used to conduct this study are $2447968.5 \mathrm{MHz}\left(\mathrm{CH}_{2} \mathrm{~F}_{2}\right) ; 2522781.5 \mathrm{MHz}\left(\mathrm{CH}_{3} \mathrm{OH}\right) ; 2546494.0$ $\mathrm{MHz}\left(\mathrm{CH}_{2} \mathrm{~F}_{2}\right) ; 2588361.8 \mathrm{MHz}\left({ }^{13} \mathrm{CH}_{3} \mathrm{OH}\right) ; 2633899.1 \mathrm{MHz}\left({ }^{13} \mathrm{CH}_{3} \mathrm{OH}\right)^{13} ; 2664058.3$ $\mathrm{MHz}\left(\mathrm{CH}_{2} \mathrm{DOH}\right)$; and $2714715.1 \mathrm{MHz}\left({ }^{13} \mathrm{CH}_{3} \mathrm{OH}\right)$. The fixed frequency FIR laser radiation is focussed onto an antenna and mixed with continuously tunable microwave radiation $(2-65 \mathrm{GHz})$ in a $1 \mathrm{~T} 12 \mathrm{GaAs}$ Schottky barrier diode to produce tunable sum and difference frequencies $\left(v_{\text {sidebands }}=v_{\text {laser }}+/-v_{\text {microwaves }}\right)$. These tunable sidebands are then separated from the stronger carrier with a Martin-Puplett diplexer. Further separation is usually accomplished with two 750 line/inch nickel meshes operating as a tunable FabryPerot etalon. As the tunable FIR laser is scanned, the etalon is adjusted to the transmission maximum for both the upper and lower sidebands, completely rejecting the FIR carrier from the detector field of view. The tunable sidebands are then collimated and multipassed 8-16 times in front of a cw supersonic slit expansion, from which the molecular beam of $\left(\mathrm{D}_{2} \mathrm{O}\right)_{2}$ is formed and cooled to a rotational temperature of $4.7 \mathrm{~K}$. Finally, the sideband radiation is detected with a liquid He cooled mechanically stressed Ge:Ga photoconductor ${ }^{14}$ as it exits the vacuum chamber, and the molecular absorptions are detected with the standard lockin detection scheme described in Chapter II.

Finally, previous measurements of these particular VRT bands were conducted with 
a two inch, supersonic expansion slit source. However, it has been shown in this laboratory that a four inch long nozzle provides adequate cooling with a minimal amount of doppler broadening. This source has become the standard cluster source for the Berkeley FIR-VRT spectrometers because of the advantage of pathlength gain relative to the two inch nozzle. The molecular beam of the water dimer- $\mathrm{d}_{4}$ is formed in this nozzle by flowing argon (99.99\% purity) through a $\mathrm{D}_{2} \mathrm{O}$ (99.9\% purity) sample. The flow rate of the mixture was adjusted to produce a backing pressure of approximately 700 Torr while a chamber pressure of $200 \mathrm{mtorr}$ is maintained by the $2800 \mathrm{cfm}$ Roots blower vacuum system.

\subsection{Results and Analysis}

\subsubsection{Group Theoretical Considerations}

In order to describe the $82.6 \mathrm{~cm}^{-1}$ VRT band system and its dynamical implications, a review of the relevant group theory is essential. In general, tunneling splittings in the rovibrational energy levels of the water dimer arise because equivalent nuclei in an initial framework may be permuted along tunneling pathways through low energy barriers, to 16 symmetrically equivalent frameworks located in adjacent minima on the multidimensional IPS of the cluster. ${ }^{15}$ Each of these frameworks are shown in Figure 3.1 along with the permutation-inversion operations required to reach a particular structure. The feasibility of each tunneling motion is related to the shape of these barriers and how they impede the conversion from the initial framework to any of the remaining 15 symmetrically equivalent frameworks on the water dimer IPS. Thus, the motion passing through the smallest barrier is the most feasible tunneling coordinate and this is responsible for the largest VRT splitting in a spectral band.

If it is assumed that all permutations of equivalent particles are feasible, the number of symmetrically equivalent frameworks is defined by the direct product group $\left[S_{2}\right] \times\left[S_{4}\right]=48$. The subgroup $\left[S_{2}\right]$ corresponds to the permutation group for the two equivalent oxygen atoms, whereas [ $\left.\mathrm{S}_{4}\right]$ allows for all possible permutations of the four 
equivalent hydrogen atoms. By including the operation which inverts all particles through the center of mass of the cluster (E*), this number is doubled to 96 , which would be the order of the complete nuclear permutation inversion group (CNPI), G96. However, within the scope of this analysis, it is assumed that the breaking of a chemical bond is not a feasible permutation, thus, the exchange of identical particles through the scrambling of hydrogen atoms between the two water monomers is not considered. In this limit the full molecular symmetry group reduces to an order which is calculated by the direct product $\left[S_{2}\right] \times\left[S_{2}\right]^{2} \times E^{*}=16$, and is therefore labeled $G_{16}$. This molecular symmetry group is isomorphic with the $D_{2 h}(M)$ point group, and is consistent with the VRT dynamical manifestations in the water dimer. Before describing each of the tunneling coordinates, a qualitative correlation diagram which elucidates the result of each motion on the $v=0, J=$ $0, K_{a}=0$ rovibrational energy level of the near-prolate asymmetric rotor is shown in the lower portion of Figure 3.2. The diagram in Figure 3.2 assumes that the rigid non-tunneling water dimer has a plane of symmetry, and may be described by the $C_{s}(M)$ point group. As the different tunneling motions are considered, the splitting of the $C_{s}(M)$ energy levels occurs until eventually the full correlation diagram in the $G_{16}$ limit is generated.

Previous studies of the water dimer have established a ground state equilibrium structure in which one water monomer acts as an hydrogen bond (H-bond) donor and the other an H-bond acceptor. ${ }^{16}$ The permutation of the two acceptor deuterium atoms, identified hereafter as acceptor tunneling, is the most feasible tunneling coordinate because it induces the largest splitting in the rovibrational energy levels. This tunneling coordinate has been proposed to be similar to a concerted internal rotation of the donor with an inversion of the acceptor, which is analogous to the inversion-internal rotation tunneling coordinate of methylamine, ${ }^{17}$ although the possibility of a simple rotation about the $\mathrm{C}_{2}$ axis of the acceptor subunit cannot be conclusively ruled out. ${ }^{5}$ Regardless of the actual tunneling pathway, the overall effect is equivalent to the rotation of the acceptor subunit about its $\mathrm{C}_{2}$ axis. The tunneling barrier for this motion in the normal isotope of the dimer 
Figure 3.1 Caption

The 16 nonsuperimposable symmetrically equivalent structures for the water dimer in the $\mathrm{G}_{16}$ molecular symmetry group are shown. The permutation-inversion operation which transforms the top structure of the left column into any of the remaining forms is located to the left of each of the labeled forms.

\section{Figure 3.2 Caption}

The correlation diagram for the $\mathrm{J}=0, \mathrm{~K}_{\mathrm{a}}=0$ rovibrational state of the water dimer is drawn to illustrate the effect of each tunneling motion on the rovibrational energy levels. The largest tunneling splitting is due to the acceptor tunneling motion, whereas the two sets of triplets arise from donor acceptor interchange tunneling. The donor tunneling motion cannot further split the energy levels and only modifies the triplet structure as shown. The symmetries alternate with $\mathrm{J}$ according to Equation 3.1 and this is used to determine the ground and excited state symmetry relating to each water dimer-d4 transition. In the upper portion of the figure, an energy level diagram is shown which represents the VRT levels of an excited vibrational state for which an arbitrary symmetry ordering is adopted to define the notation used throughout the text. The energy differences of the center of the ground and excited state interchange triplets are defined as the fitted band origins. The "upper", "E", and "lower" transitions correspond to the highest, center, and lowest frequency bands, whereas the $(+)$ and $(-)$ describe the highest and lowest energy components of each interchange triplets. The $(+),(-)$ notation is applied regardless of the symmetry labels assigned to the VRT levels in the cluster. The notation regarding the symmetry species of the interchange triplets have been condensed so that $A_{1}+/-/ E^{+/-/ B_{1}}+/-=" 1 "$ and $A_{2}-1+/ E^{-}$ $1+/ \mathrm{B}_{2}{ }^{-1+}=" 2 "$, as is indicated at the right hand side of the diagram. 
Figure 3.1

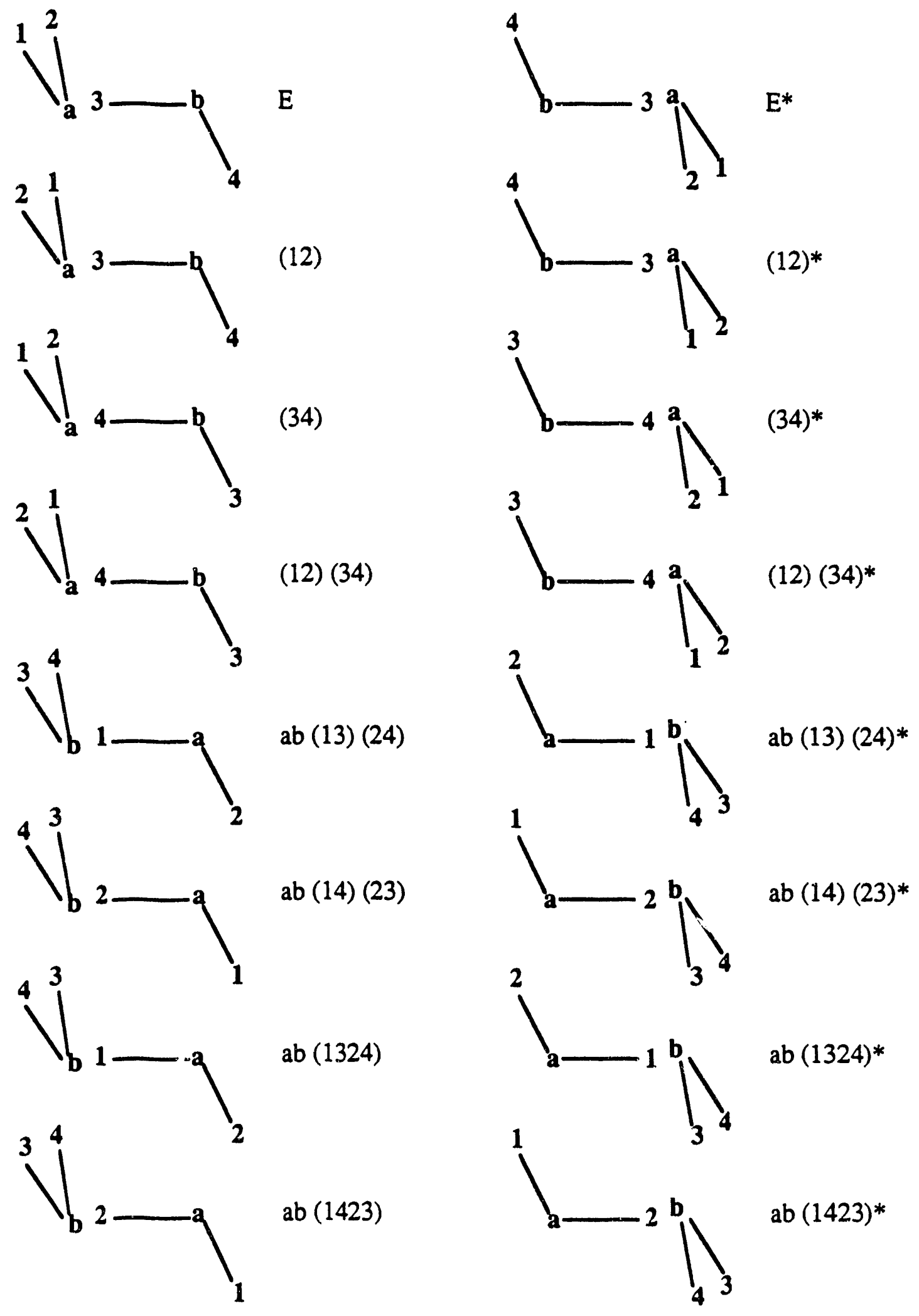


Figure 3.2

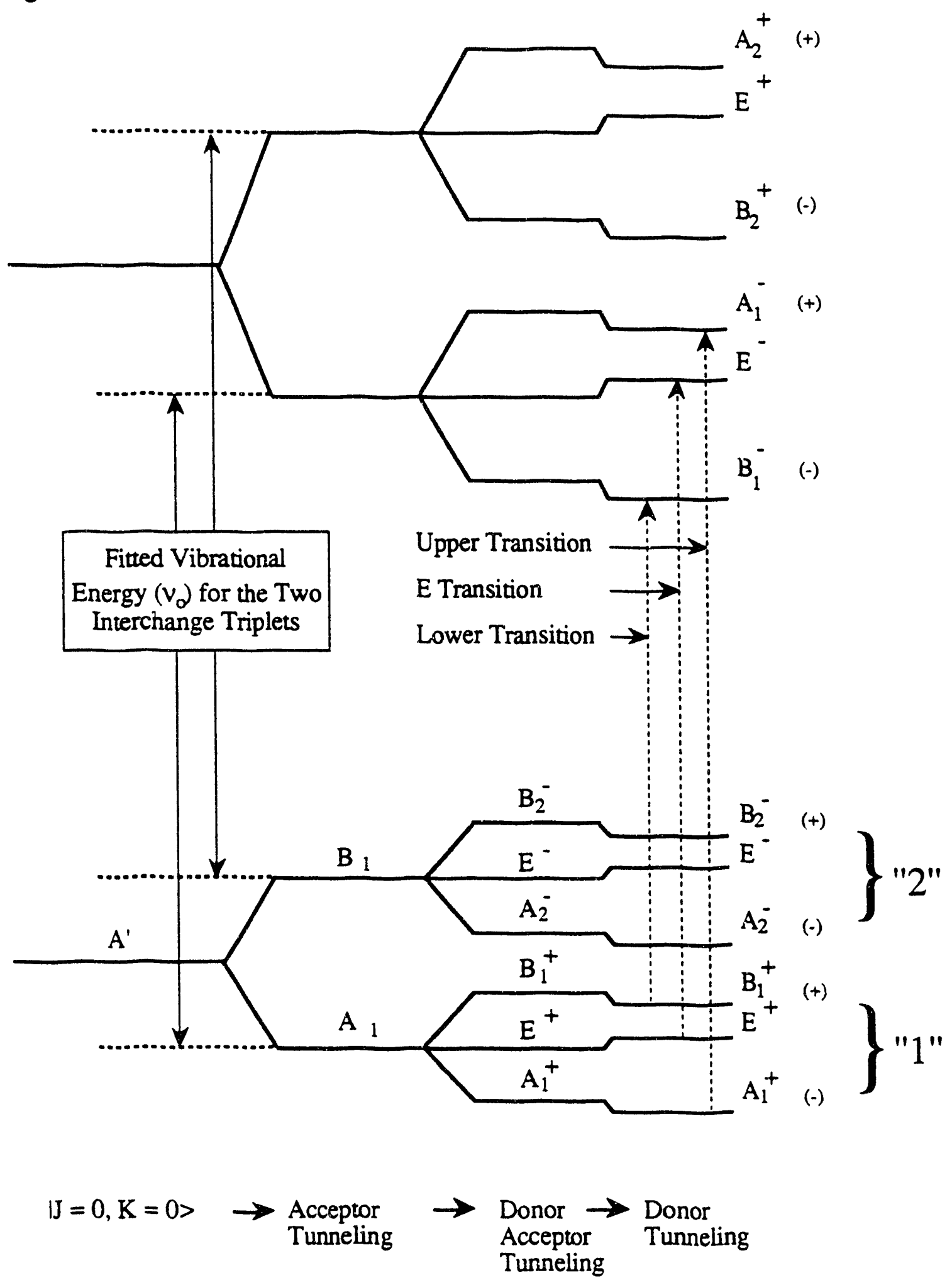

$$
\mathrm{C}_{s}(\mathrm{M}) \quad \mathrm{C}_{2 \mathrm{v}}(\mathrm{M}) \quad \mathrm{G}_{16}=\mathrm{D}_{2 \mathrm{~h}}(\mathrm{M})
$$


along the proposed methylamine-like pathway has been estimated 5 to be $130 \mathrm{~cm}^{-1}$ by examining the intermolecular PES of Coker and Watts. ${ }^{18}$ However, the transition state along the methylamine-like acceptor tunneling pathway is found to have a somewhat higher barrier of $206 \mathrm{~cm}-1$ when calculated at the MP2/6-31+G(d,p) level of theory by Smith et a1. ${ }^{19}$ The effect of acceptor tunneling through this low IPS barrier splits each rovibrational energy level of the non-tunneling water dimer into two discrete VRT states, and the appropriate symmetry group for the cluster is $C_{2} v(M)$ in this limit. Thus, rovibrational states of $A^{\prime}$ and $A^{\prime \prime}$ symmetry in the $C_{\mathfrak{s}}(M)$ point group correlate with VRT states having $A_{1} / B_{1}$ and $A_{2} / B_{2}$ symmetries, respectiveiy, in $C_{2} v(M)$ and this correlation is shown in Figure 3.2.

The next most feasible tunneling coordinate corresponds to donor-acceptor interchange, for which many pathways could conceivably exist, although a geared interconversion, similar to that occurring in the HF dimer ${ }^{20}$ is possibly the most feasible. An estimate of approximately $80 \mathrm{u} \mathrm{cm}^{-1} \mathrm{fr}, \mathrm{r}$ this barrier has been reported in Reference 5 for the normal isotopomer of the water dimer, however, the ab initio calculations of Smith et al. 22 calculate a cyclic transition structure of $C_{i}$ symmetry, at an energy of $304 \mathrm{~cm}^{-1}$ for this pathway. Regardless of the disagreement between the two predicted barriers, this tunneling motion should produce a splitting of each rovibrational energy level that is smaller than that for acceptor tunneling, for these donor-acceptor interchange barriers are at least a factor of three higher. The presence of donor-acceptor interchange tunneling further splits the VRT levels from the $C_{2 v}(M)$ limit - in which only the acceptor tunneling motion is feasible - and the manifestation of this motion on the splitting pattern is most clearly understood by examining line correlation diagram in Figure 3.2. In particular, the $A_{1}, B_{1}$, $A_{2}$, and $B_{2}$ symmetry species in $C_{2 v}(M)$ split into VRT states having symmetries of $\mathrm{A}_{1}+/ \mathrm{E}^{+} / \mathrm{B}_{1}{ }^{+}, \mathrm{A}_{2}^{-} / \mathrm{E}^{-} / \mathrm{B}_{2}^{-}, \mathrm{A}_{1}^{-} / \mathrm{E}^{-/ \mathrm{B}_{1}}$, and $\mathrm{A}_{2}^{+} / \mathrm{E}^{+} / \mathrm{B}_{2}{ }^{+}$, respectively. The direct product of the PI operations which describe this motion with those for acceptor tunneling, permit an initial framework of the dimer to access all of the 16 nonsuperimposable, symmetrically 
equivalent frameworks corresponding to the IPS minima of the water dimer. These two tunneling motions fully resolve all possible degeneracies for each rovibrational energy level and define the appropriate molecular symmetry group of the cluster to be $G_{16}\left(=D_{2 h}(M)\right)$. The correlation from $C_{s}(M)$ to $G_{16}$ is

$$
\begin{aligned}
& \Gamma_{\mathrm{A}^{\prime}}=\mathrm{A}_{1^{+}}(7)+\mathrm{E}^{+}(3)+\mathrm{B}_{1}^{+}(5)+\mathrm{A}_{2}^{-}(1)+\mathrm{E}^{-}(3)+\mathrm{B}_{2}^{-}(2) \\
& \Gamma_{\mathrm{A}^{\prime \prime}}=\mathrm{B}_{1^{-}}(5)+\mathrm{E}^{-}(3)+\mathrm{A}_{1^{-}}(7)+\mathrm{B}_{2}^{+}(2)+\mathrm{E}^{+}(3)+\mathrm{A}_{2}^{+}(1),
\end{aligned}
$$

in which the numbers in parentheses correspond to the relative nuclear spin statistical weights for each symmetry species of the perdeuterated water dimer. The vertical ordering in Equation 3.1 indicates how the symmetries of the VRT energy levels alternate with J. For example, in $\mathrm{K}_{\mathrm{a}}=0$, even $\mathrm{J}$ correlate to the $\Gamma_{\mathrm{A}^{\prime}}$ symmetries and odd $\mathrm{J}$ correlate with $\Gamma_{\mathrm{A}^{n}}$, and a particular component of the VRT splitting pattern alternates between symmetry species within the vertical columns of Equation 3.1. Applying the electric dipole selection rule of $\Gamma^{+}<\rightarrow \Gamma^{-}$, the splitting pattern diagramed in Figure 3.1 predicts six possible VRT subbands for the conditions in which $\Delta \mathrm{K}_{\mathrm{a}}=0$ or $+/-1$. Three of these six VRT transitions are qualitatively shown in Figure 3.2 for an hypothetical $\Delta \mathrm{K}_{\mathrm{a}}=0$, a-type band. The excitation of the vibrational coordinate at $82.6 \mathrm{~cm}^{-1}$ provides a precise measure of the change in each of the tunneling splittings from the ground to the excited state, and the detailed knowledge of the tunneling splittings in $v=0$ can be utilized in characterizing the excited state dynamics.

In their IAM fit of $\left(\mathrm{H}_{2} \mathrm{O}\right)_{2}$, Coudert and Hougen ${ }^{9}$ have actually proposed two types of interconversion pathways to account for the observed interconversion splittings in the ground state. These two pathways resemble geared and antigeared concerted internal rotations of the two water subunits. In this model, the splitting between the $A^{+/-/ B+/-V R T ~}$ levels for the interchange triplet labeled with $A_{1}+/ / E^{+/ /} / B_{1}+/$ symmetries is determined to be the sum of the two tunneling matrix elements for the geared and antigeared interchange motions, whereas the analogous splitting for the interchange triplet represented by the 
$\mathrm{A}_{2} 2^{-1+/ \mathrm{E}^{-/+} / \mathrm{B}_{2}}{ }^{-1+}$ symmetry labels is characterized by the difference of these matrix elements. This interpretation has been used to fit the interchange tunneling splitting so that the larger of the two is labeled with $A_{1}+/ / E^{+/-/} / B_{1}+/$. . However, in the $82.6 \mathrm{~cm}^{-1}$ vibration presented in this chapter, the IAM description of the ground state cannot be extended, because the larger interchange splitting is assigned with $A_{2}-1+/ E^{-/+} / B_{2}-/+$ symmetry labels and the smaller splitting corresponds to states of $A_{1}+/-/ E^{+/-/} / B_{1}+/$ symmetry. Thus, for the purpose of this study the interchange coordinate is simply fit as a single tunneling parameter which describes the splitting between each of the A/B VRT states, and the matrix element notation of the IAM model ${ }^{4,5.9}$ (such as $\mathrm{h}_{5}$ and $\mathrm{h}_{7}$ corresponding to geared and antigeared motions, respectively) is avoided.

Finally, the permutation of the equivalent nuclei on the donor monomer (donor tunneling) further modifies the energy level diagram but introduces no additional tunneling splittings, and is fit to a tunneling parameter which effectively shifts the middle VRT state of $E$ symmetry away from the center of each donor-acceptor interchange triplet. Barrier heights for this motion in $\left(\mathrm{H}_{2} \mathrm{O}\right)_{2}$ are found to be ca. $1000 \mathrm{~cm}^{-1}$ from the estimate of Coudert and Hougen ${ }^{5}$, and $658 \mathrm{~cm}^{-1}$ in the ab-initio calculations of Smith et al..$^{22}$ These reflect a pathway which requires the breaking of the $5 \mathrm{kcal} \mathrm{H}$-bond, resulting in the relatively small spectral shift qualitatively shown in Figure 3.2. The three tunneling pathways described in this section are illustrated in Figure 3.3.

\subsubsection{Spectroscopic Implications and Notation Definitions}

The electric dipole selection rule $\Gamma^{+} \leftrightarrow \rightarrow \Gamma^{-}$forbids transitions across the acceptor and donor tunneling splittings and only the effect of relative changes in these tunneling coordinates can be extracted from the data. This selection rule governs the symmetry of the VRT state in the vibration to which each transition terminates; thus when possible, the relative nuclear spin statistical weights listed in Equation 3.1 are used to determine the symmetry assignment of a particular excited state VRT symmetry species. Formally, 
Figure 3.3

(a) Acceptor Tunneling

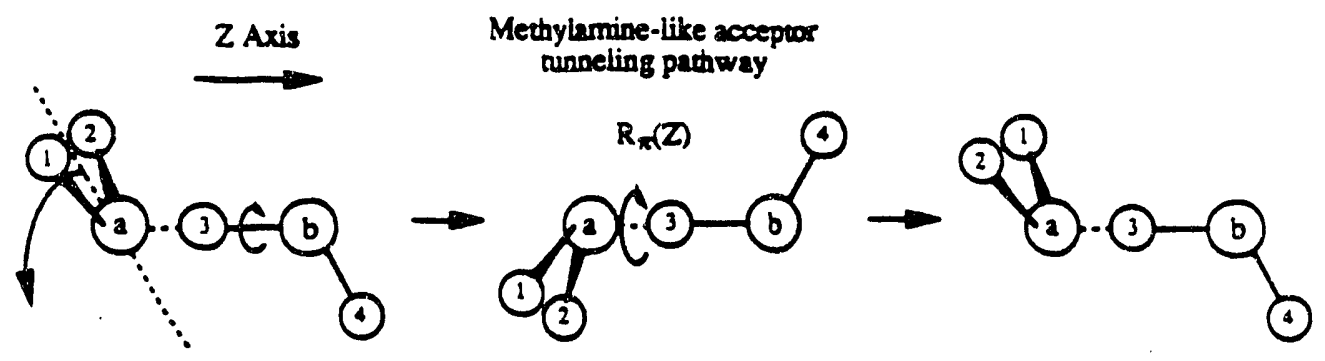

(b) Geared Donor-Acceptor Interconversion Tunneling

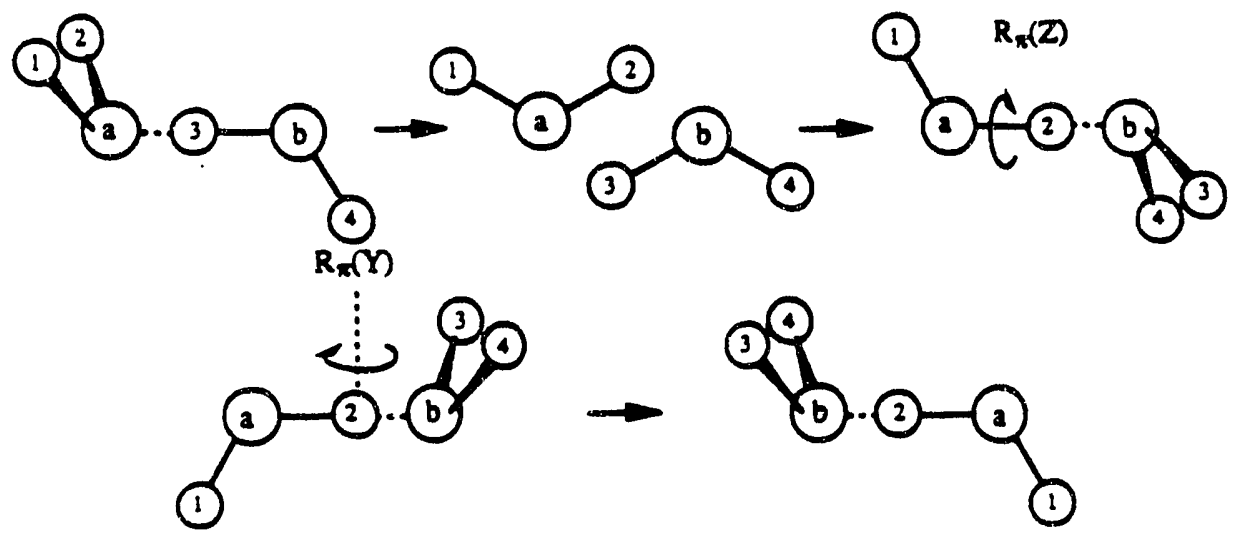

(c) Donor Tunneling

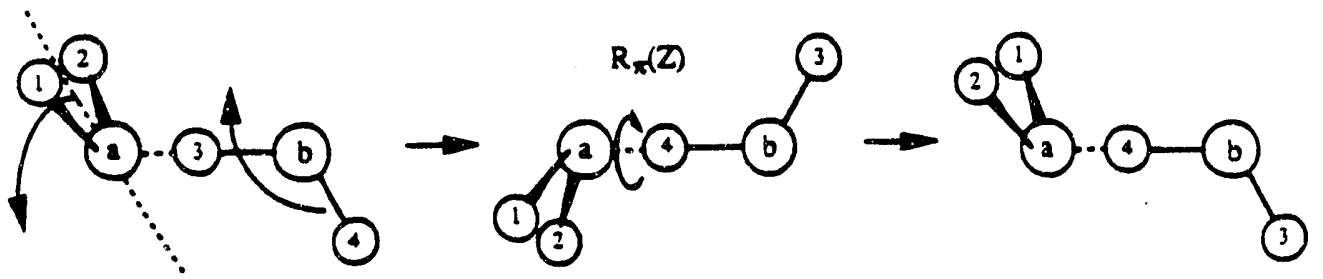

This figure illustrates the pathways for the methylamine-like acceptor tunneling, geared donor-acceptor interconversion tunneling, and donor tunneling. Each of these tunneling pathways begin with an identical labeled form of the dimer, and end up in a distinct labeled form. The appropriate equivalent rotations are included to rectum the dimer to its original orientation.

61 
transitions between states of $E$ symmetry are allowed between the two $A_{1}+/-/ E^{+/-/} / B_{1}+/-$ and

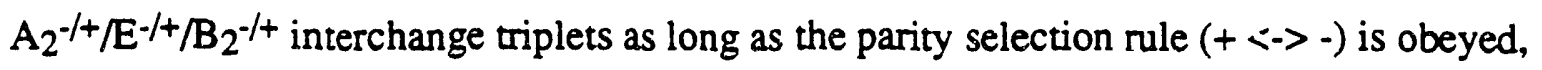
however, no transitions of this type have been detected to date in the water dimer.

The entire spectral range from 2408 to $2758 \mathrm{GHz}$ has been continuously scanned and approximately 230 transitions have been assigned to 12 VRT bands, all of which originate from the $\mathrm{K}_{\mathrm{a}}{ }^{\prime \prime}=0$ manifold and terminate in either $\mathrm{K}_{\mathrm{a}}{ }^{\prime}=0$ or 1 . All of the transitions assigned to these VRT bands are reported in Table 3.1. The $\mathrm{K}_{\mathrm{a}}{ }^{\prime}=0->0$ parallel transitions from the microwave data of Suenram et al. ${ }^{21}$ are also listed and are weighted in the fit according to the precision of these data, $(4 \mathrm{kHz}$ and $100 \mathrm{kHz}$ for transitions observed with the FTMW and EROS instruments, respectively), whereas each FIR transition is assigned a precision of $3.0 \mathrm{MHz}$. As the tunneling parameters and notational considerations are discussed below, attention should be direcred to the remaining features of Figure 3.2 to clarify the meaning of a particular definition. In fitting each VRT subband, the end-over-end rotational motion is described by the expression

$$
E=v_{0}+B J(J+1)-D(J(J+1))^{2}+H(J(J+1))^{3}+(-1)^{J+K a+K c}[((B-C) / 4)(J(J+1))] .
$$

The vibrational energy $\left(v_{0}\right)$, in Equation 3.2 is defined as the average energy of the $A / B$ VRT levels such that the two separate interchange triplets (which transform as the symmetry species $A_{1}+/-/ E^{+/-/ B_{1}}+/-$ and $A_{2}-1+/ E^{-1+/ B_{2}} 2^{-/+}$) of the ground vibrational state are both assigned a value of $v_{0}=0$, and the two excited state interchange triplets for each value of $\mathrm{K}_{\mathrm{a}}$ are fit to yield unique band origins. The definition of this energy contribution is pictorially shown in Figure 3.2. This is done for convenience so that the description of the $82.6 \mathrm{~cm}^{-1}$ VRT subbands is more concise, and can be discussed as a set of transitions corresponding to a particular donor-acceptor interchange triplet. The difference of the two fitted band origins for either the $\Delta \mathrm{K}_{\mathrm{a}}=0$ or 1 transitions corresponds to the change in the 
Table 3.1

All transitions involved in the present analysis are reported (in $\mathrm{MHz}$ ). The microwave $\mathrm{v}=$ $0, K_{a}=0 \rightarrow 0$ transitions from Reference 24 are also included in the uppermost portion of the table. The excited vibrational state data are separated for the $K_{a}=0 \rightarrow 0$ and $K_{a}=0 \rightarrow$ 1 VRT subbands. These subbands are organized so that the transitions characterized by symmetry species " 1 " and " 2 " are positioned on the left and right sides of the pages, respectively, for the lower, E, and upper bands. The method of fitting the bands is described in Section III of this work.

Ground State $\mathrm{K}=0$ Microwave Transitions

\begin{tabular}{lllll}
\hline \hline$\Gamma^{i}$ & $J^{\prime}$ & $J^{\prime \prime}$ & Frequency & $\mathrm{O}-\mathrm{C}$ \\
\hline \hline $\mathrm{A}_{1}{ }^{+}$ & 1 & 0 & 12036.274 & 0.036 \\
$\mathrm{~A}_{1}^{-}$ & 2 & 1 & 20557.879 & -0.006 \\
$\mathrm{~A}_{1}^{+}$ & 3 & 2 & 33758.33 & -0.020 \\
$\mathrm{~A}_{1}^{-}$ & 4 & 3 & 42284.43 & 0.020 \\
$\mathrm{~A}_{1}^{-}$ & 6 & 5 & 64000.45 & 0.020 \\
$\mathrm{E}^{+/-}$ & 1 & 0 & 10864.491 & -0.024 \\
$\mathrm{E}^{-/+}$ & 2 & 1 & 21728.171 & -0.004 \\
$\mathrm{E}^{+/-}$ & 3 & 2 & 32590.14 & 0.020 \\
$\mathrm{E}^{-/+}$ & 4 & 3 & 43449.50 & 0.040 \\
$\mathrm{E}^{-/+}$ & 6 & 5 & 65156.54 & -0.130 \\
$\mathrm{E}^{+/-}$ & 7 & 6 & 76002.73 & 0.080 \\
$\mathrm{E}^{-/+}$ & 10 & 9 & 108497.45 & 0.000 \\
$\mathrm{~B}_{1}^{+}$ & 1 & 0 & 9692.870 & -0.014 \\
$\mathrm{~B}_{1}^{-}$ & 2 & 1 & 22898.617 & 0.000 \\
$\mathrm{~B}_{1}^{+}$ & 3 & 2 & 31422.00 & -0.010 \\
$\mathrm{~B}_{1}^{-}$ & 4 & 3 & 44614.57 & -0.030 \\
$\mathrm{~B}_{1}^{-}$ & 6 & 5 & 66313.13 & 0.020 \\
$\mathrm{~B}_{1}{ }^{+}$ & 7 & 6 & 74852.29 & -0.030 \\
$\mathrm{~A}_{2}^{-}$ & 1 & 0 & 11947.563 & 0.006 \\
$\mathrm{~A}_{2}^{+}$ & 2 & 1 & 20646.290 & 0.020 \\
$\mathrm{~A}_{2}^{-}$ & 3 & 2 & 33670.81 & -0.010 \\
$\mathrm{~A}_{2}^{+}$ & 4 & 3 & 42371.73 & 0.020 \\
$\mathrm{~B}_{2}^{-}$ & 1 & 0 & 9781.609 & 0.025 \\
$\mathrm{~B}_{2}^{+}$ & 2 & 1 & 22810.364 & 0.021 \\
$\mathrm{~B}_{2}^{-}$ & 3 & 2 & 31509.87 & -0.05 \\
$\mathrm{~B}_{2}^{+}$ & 4 & 3 & 44528.18 & 0.00 \\
& & & &
\end{tabular}

$\mathrm{K}_{\mathrm{a}}=0 \rightarrow 0$ Lower Bands

\begin{tabular}{|c|c|c|c|c|c|c|c|c|c|}
\hline$\Gamma \mathrm{i}$ & $\mathrm{J}^{\prime}$ & $\mathrm{J}^{\prime \prime}$ & Frequency & $\mathrm{O}-\mathrm{C}$ & $\Gamma i$ & $\overline{\mathrm{J}^{\prime}}$ & $\mathrm{J}^{\prime \prime}$ & Frequency & $\overline{\mathrm{O}-\mathrm{C}}$ \\
\hline $\mathrm{B}_{1}^{+}$ & 5 & 6 & 2408772.2 & -0.6 & $\mathrm{~B}_{2}^{-}$ & 5 & 6 & 2409166.3 & 1.1 \\
\hline $\mathrm{A}_{1}^{-}$ & 4 & 5 & 2419997.1 & -1.6 & $\mathrm{~A}_{2}^{+}$ & 4 & 5 & 2420271.2 & 0.2 \\
\hline $\mathrm{B} 1+$ & 3 & 4 & 2431159.0 & 0.5 & $\mathrm{~B}_{2}$ & 3 & 4 & 2431329.6 & -0.3 \\
\hline$A_{1}^{-}$ & 2 & 3 & 2442246.1 & -1.9 & $\mathrm{~A}_{2}^{+}$ & 2 & 3 & 2442338.6 & -2.8 \\
\hline
\end{tabular}




$\begin{array}{llllllllll}\mathrm{B}_{1}^{+} & 1 & 2 & 2453263.2 & -1.4 & \mathrm{~B}_{2}^{-} & 1 & 2 & 2453305.7 & 1.0 \\ \mathrm{~A}_{1}^{-} & 0 & 1 & 2464203.9 & -1.8 & \mathrm{~B}_{2}^{-} & 1 & 0 & 2485897.2 & 0.6 \\ \mathrm{~B} 1+^{-} & 1 & 0 & 2485862.7 & 6.5 & \mathrm{~A}_{2}^{+} & 2 & 1 & 2496658.2 & -0.3 \\ \mathrm{~A}_{1}^{-} & 2 & 1 & 2496564.9 & 0.5 & \mathrm{~B}_{2}^{-} & 3 & 2 & 2507368.4 & 0.3 \\ \mathrm{~B}_{1}^{+} & 3 & 2 & 2507194.0 & -1.0 & \mathrm{~A}_{2}^{+} & 4 & 3 & 2518022.9 & -1.3 \\ \mathrm{~A}_{1}^{-} & 4 & 3 & 2517748.7 & -0.9 & \mathrm{~B}_{2}^{-} & 5 & 4 & 2528628.4 & 2.3 \\ \mathrm{~B}_{1}^{+} & 5 & 4 & 2528230.3 & 0.1 & \mathrm{~A}_{2}^{+} & 6 & 5 & 2539173.3 & 0.7 \\ \mathrm{~A}_{1}^{-} & 6 & 5 & 2538641.2 & 1.8 & \mathrm{~B}_{2}^{-} & 7 & 6 & 2549663.1 & 0.4 \\ \mathrm{~B}_{1}^{+} & 7 & 6 & 2548979.7 & -0.9 & \mathrm{~A}_{2}^{+} & 8 & 7 & 2560094.0 & -1.4 \\ \mathrm{~A}_{1}^{-} & 8 & 7 & 2559258.3 & 0.0 & \mathrm{~B}_{2}^{-} & 9 & 8 & 2570467.5 & -2.1 \\ \mathrm{~B}_{1}^{+} & 9 & 8 & 2569479.7 & 2.2 & \mathrm{~A}_{2}^{+} & 10 & 9 & 2580785.9 & 1.5 \\ \mathrm{~A}_{1}^{-} & 10 & 9 & 2579642.3 & -1.3 & & & & & \end{array}$

$\mathrm{K}_{\mathrm{a}}=0 \rightarrow 0 \mathrm{E}$ Band

\begin{tabular}{|c|c|c|c|c|c|c|c|c|c|}
\hline$\Gamma \mathrm{i}$ & $\overline{J^{\prime}}$ & $J^{\prime \prime}$ & Frequency & $\mathrm{O}-\mathrm{C}$ & $\Gamma_{i}$ & $\bar{J}^{\prime}$ & J" & Frequency & $\overline{O-C}$ \\
\hline$E^{+}$ & 5 & $\overline{6}$ & 2411430.0 & 1.1 & E- & 5 & 6 & 2411533.2 & $\overline{0.2}$ \\
\hline$E^{-}$ & 4 & 5 & 2422633.5 & 0.1 & $E^{+}$ & 4 & 5 & 2422638.4 & -0.9 \\
\hline$E^{+}$ & 3 & 4 & 2433776.1 & 0.8 & $E^{-}$ & 3 & 4 & 2433696.6 & -2.1 \\
\hline$E^{+}$ & 1 & 2 & 2455860.0 & 1.4 & $E^{+}$ & 2 & 3 & 2444710.9 & 0.3 \\
\hline$E^{-}$ & 0 & 1 & 2466797.6 & 2.3 & $E^{-}$ & 1 & 2 & 2455673.1 & -0.9 \\
\hline$E^{+}$ & 1 & 0 & 2488450.9 & -0.3 & $E^{+}$ & 0 & 1 & 2466590.3 & 1.8 \\
\hline$E^{-}$ & 2 & 1 & 2499167.7 & -1.8 & $E^{-}$ & 1 & 0 & 2488267.3 & 0.5 \\
\hline$E^{+}$ & 3 & 2 & 2509814.7 & -0.1 & $E^{+}$ & 2 & 1 & 2499030.1 & 1.2 \\
\hline$E^{-}$ & 4 & 3 & 2520381.1 & -6.9 & $\mathrm{E}^{-}$ & 3 & 2 & 2509738.4 & 0.0 \\
\hline$E^{+}$ & 5 & 4 & 2530892.3 & 1.5 & $E^{+}$ & 4 & 3 & 2520390.6 & -3.4 \\
\hline$E^{-}$ & 6 & 5 & 2541327.1 & 2.0 & $E^{-}$ & 5 & 4 & 2530996.8 & 1.9 \\
\hline$E^{+}$ & 7 & 6 & 2551693.8 & 0.0 & $E^{+}$ & 6 & 5 & 2541542.0 & 2.3 \\
\hline$E^{-}$ & 8 & 7 & 2561999.5 & -0.9 & $E^{-}$ & 7 & 6 & 2552025.9 & -1.3 \\
\hline $\mathrm{E}^{+}$ & 9 & 8 & 2572250.5 & 1.2 & $E^{+}$ & 8 & 7 & 2562456.9 & 0.3 \\
\hline$E^{-}$ & 10 & 9 & 2582444.6 & -0.7 & $E^{-}$ & 9 & 8 & 2572826.9 & 0.4 \\
\hline & & & & & $\mathrm{E}^{+}$ & 10 & 9 & 2583135.7 & -0.4 \\
\hline
\end{tabular}

$\mathrm{K}_{\mathrm{a}}=0 \rightarrow 0$ Upper Bands

\begin{tabular}{|c|c|c|c|c|c|c|c|c|c|}
\hline$\Gamma \mathrm{i}$ & $J^{\prime}$ & & Frequency & $\mathrm{O}-\mathrm{C}$ & $\Gamma i$ & $\overline{J^{\prime}}$ & $\overline{J^{\prime \prime}}$ & Frequency & $\overline{\mathrm{O}-\mathrm{C}}$ \\
\hline $\mathrm{A}_{1}{ }^{+}$ & 5 & 6 & 2413994.1 & -0.9 & $\mathrm{~A}_{2}^{-}$ & 5 & 6 & 2413615.7 & $\overline{\overline{3.1}}$ \\
\hline$B_{1}^{-}$ & 4 & 5 & 2425179.9 & -0.9 & $\mathrm{~B}_{2}^{+}$ & 4 & 5 & 2424710.1 & -2.4 \\
\hline $\mathrm{A}_{1}^{+}$ & 3 & 4 & 2436306.6 & -0.4 & $\mathrm{~A}_{2}^{-}$ & 3 & 4 & 2435765.0 & -2.0 \\
\hline $\mathrm{A}_{1}^{+}$ & 1 & 2 & 2458372.8 & 2.5 & $\mathrm{~A}_{2}^{-}$ & 1 & 2 & 2457735.4 & -1.1 \\
\hline$B_{1}^{-}$ & 0 & 1 & 2469307.6 & 4.5 & $\mathrm{~B}_{2}^{+}$ & 0 & 1 & 2468646.8 & -3.1 \\
\hline $\mathrm{A}_{1}+$ & 1 & 0 & 2490963.6 & -0.7 & $\mathrm{~A}_{2}^{-}$ & 1 & 0 & 2490329.5 & -0.8 \\
\hline $\mathrm{B}_{1}^{-}$ & 2 & 1 & 2501692.4 & 0.7 & $\mathrm{~B}_{2}+$ & 2 & 1 & 2501097.1 & 1.5 \\
\hline
\end{tabular}




$\begin{array}{llllllllll}\mathrm{A}_{1}^{+} & 3 & 2 & 2512350.3 & 0.4 & \mathrm{~A}_{2}^{-} & 3 & 2 & 2511812.7 & 3.1 \\ \mathrm{~B}_{1^{-}} & 4 & 3 & 2522941.4 & 1.8 & \mathrm{~B}_{2}^{+} & 4 & 3 & 2522475.6 & 4.0 \\ \mathrm{~A}_{1}^{+} & 5 & 4 & 2533456.3 & -5.6 & \mathrm{~A}_{2}^{-} & 5 & 4 & 2533081.3 & 0.8 \\ \mathrm{~B}_{1}^{-} & 6 & 5 & 2543915.3 & -3.0 & \mathrm{~B}_{2}^{+} & 6 & 5 & 2543636.2 & 0.9 \\ \mathrm{~A}_{1}^{+} & 7 & 6 & 2554306.4 & -4.5 & \mathrm{~A}_{2}^{-} & 7 & 6 & 2554135.1 & 0.0 \\ \mathrm{~B}_{1^{-}}^{-} & 8 & 7 & 2564641.6 & -0.4 & \mathrm{~B}_{2}^{+} & 8 & 7 & 2564573.7 & -4.9 \\ \mathrm{~A}_{1}^{+} & 9 & 8 & 2574916.0 & 1.3 & \mathrm{~A}_{2}^{-} & 9 & 8 & 2574962.9 & -2.2 \\ \mathrm{~B}_{1^{-}} & 10 & 9 & 2585134.5 & 2.3 & \mathrm{~B}_{2}^{+} & 10 & 9 & 2585296.4 & 3.1 \\ \mathrm{~A}_{1}^{+} & 11 & 10 & 2595302.5 & 4.0 & & & & & \\ \mathrm{~B}_{1}^{-} & 12 & 11 & 2605419.2 & 1.1 & & & & & \\ \mathrm{~A}_{1}^{+} & 13 & 12 & 2615496.3 & 0.6 & & & & & \\ \mathrm{~B}_{1^{-}} & 14 & 13 & 2625533.7 & -2.9 & & & & & \end{array}$

$\mathrm{K}=0 \rightarrow 1$ Lower Bands

\begin{tabular}{|c|c|c|c|c|c|c|c|c|c|}
\hline$\Gamma \mathrm{i}$ & $\overline{J^{\prime}}$ & $\mathrm{J}^{\prime \prime}$ & Frequency & $\mathrm{O}-\mathrm{C}$ & $\Gamma i$ & $\mathrm{~J}^{\prime}$ & $\mathrm{J}^{\prime \prime}$ & Frequency & $\overline{O-C}$ \\
\hline $\mathrm{B}_{1}^{+}$ & 5 & 6 & 2488366.0 & -1.3 & $\mathrm{~A}_{2}+$ & 6 & 7 & $2544848.1^{*}$ & $\overline{0.8}$ \\
\hline$A_{1}^{-}$ & 4 & 5 & 2499120.9 & 3.8 & $\mathrm{~B}_{2}^{-}$ & 5 & 6 & $2556500.9 *$ & 0.8 \\
\hline $\mathrm{B}_{1}^{+}$ & 3 & 4 & 2509895.1 & 3.1 & $\mathrm{~A}_{2}^{+}$ & 4 & 5 & $2568014.4^{*}$ & 0.0 \\
\hline $\mathrm{A}_{1}^{-}$ & 2 & 3 & 2520693.5 & 2.7 & $\mathrm{~B}_{2}^{-}$ & 3 & 4 & $2579392.7 *$ & -3.1 \\
\hline $\mathrm{B}_{1}^{+}$ & 1 & 2 & 2531509.6 & -2.7 & $\mathrm{~B}_{2}^{-}$ & 1 & 2 & $2601766.1^{*}$ & 0.1 \\
\hline$A_{1}^{-}$ & 1 & 1 & 2553175.9 & -4.4 & $\mathrm{~A}_{2}^{+}$ & 1 & 1 & 2623165.5 & 1.2 \\
\hline $\mathrm{B}_{1}^{+}$ & 2 & 2 & 2553097.9 & -3.7 & $\mathrm{~B}_{2}^{-}$ & 2 & 2 & 2623369.6 & 0.3 \\
\hline $\mathrm{A}_{1}^{-}$ & 3 & 3 & 2552980.9 & -2.8 & $\mathrm{~A}_{2}^{+}$ & 3 & 3 & 2623674.1 & -0.2 \\
\hline $\mathrm{B}_{1}^{+}$ & 4 & 4 & 2552825.4 & -1.5 & $\mathrm{~B}_{2}^{-}$ & 4 & 4 & 2624079.6 & -0.3 \\
\hline$A_{1}^{-}$ & 5 & 5 & 2552630.8 & -0.7 & $\mathrm{~A}_{2}^{+}$ & 5 & 5 & 2624584.7 & 0.8 \\
\hline $\mathrm{B}_{1}^{+}$ & 6 & 6 & 2552397.5 & -0.5 & $\mathrm{~B}_{2}^{-}$ & 6 & 6 & 2625188.5 & 0.5 \\
\hline$A_{1}^{-}$ & 7 & 7 & 2552123.5 & -3.5 & $\mathrm{~A}_{2}^{+}$ & 7 & 7 & 2625892.1 & 1.0 \\
\hline $\mathrm{B}_{1}^{+}$ & 8 & 8 & 2551825.3 & 6.2 & $\mathrm{~B}_{2}^{-}$ & 8 & 8 & 2626688.5 & -0.8 \\
\hline $\mathrm{A}_{1}^{-}$ & 9 & 9 & 2551473.5 & -1.2 & $\mathrm{~A}_{2}^{+}$ & 9 & 9 & 2627583.3 & -1.2 \\
\hline $\mathrm{B}_{1}^{+}$ & 1 & 0 & 2564102.2 & -1.6 & $\mathrm{~B}_{2}^{-}$ & 10 & 10 & 2628564.9 & 1.3 \\
\hline$A_{1}^{-}$ & 2 & 1 & 2575010.1 & 3.0 & $\mathrm{~A}_{2}{ }^{+}$ & 11 & 11 & 2629642.3 & 0.7 \\
\hline $\mathrm{B}_{1}^{+}$ & 3 & 2 & 2585934.7 & 6.1 & $\mathrm{~B}_{2}^{-}$ & 3 & 2 & $2655434.3^{*}$ & 0.8 \\
\hline$A_{1}^{-}$ & 4 & 3 & 2596873.3 & 5.3 & $\mathrm{~A}_{2}^{+}$ & 4 & 3 & $2665771.6 *$ & 3.7 \\
\hline $\mathrm{B}_{1}^{+}$ & 5 & 4 & 2607826.7 & 2.0 & $\mathrm{~B}_{2}^{-}$ & 5 & 4 & $2675959.0 *$ & -1.2 \\
\hline \multirow[t]{3}{*}{$A_{1}^{-}$} & 6 & 5 & 2618790.1 & -8.0 & $\mathrm{~A}_{2}^{+}$ & 6 & 5 & $2686003.0 *$ & -3.7 \\
\hline & & & & & $\mathrm{B}_{2}^{-}$ & 7 & 6 & $2695903.6 *$ & 2.2 \\
\hline & & & & & $\mathrm{A}_{2}{ }^{+}$ & 8 & 7 & $2705635.7^{*}$ & -0.5 \\
\hline
\end{tabular}

$\mathrm{K}_{\mathrm{a}}=0 \rightarrow 1 \mathrm{E}$ Bands

\begin{tabular}{lllllllllll}
\hline \hline$\Gamma_{\mathrm{i}}$ & $J^{\prime}$ & $J^{\prime \prime}$ & Frequency & $\mathrm{O}-\mathrm{C}$ & & \multicolumn{1}{l}{$\Gamma_{\mathrm{i}}$} & $\mathrm{J}^{\prime}$ & $\mathrm{J}^{\prime \prime}$ & Frequency & $\mathrm{O}-\mathrm{C}$ \\
\hline \hline $\mathrm{E}^{-}$ & 6 & 7 & 2483685.8 & 4.3 & & $\mathrm{E}^{-}$ & 1 & 2 & $2604697.8^{*}$ & -17.9
\end{tabular}




$\begin{array}{llllllllll}\mathrm{E}^{+} & 5 & 6 & 2494466.8 & 2.6 & \mathrm{E}^{+} & 2 & 3 & 2593555.2^{*} & -46.4 \\ \mathrm{E}^{-} & 4 & 5 & 2505263.4 & 3.4 & \mathrm{E}^{-} & 3 & 4 & 2582678.1^{*} & 34.2 \\ \mathrm{E}^{+} & 3 & 4 & 2516074.0 & 4.4 & \mathrm{E}^{+} & 4 & 5 & 2570859.4^{*} & -67.0 \\ \mathrm{E}^{-} & 2 & 3 & 2526896.4 & 3.3 & \mathrm{E}^{-} & 5 & 6 & 2559299.8 * & -38.2 \\ \mathrm{E}^{+} & 1 & 2 & 2537733.7 & 3.0 & \mathrm{E}^{+} & 6 & 7 & 2547591.8 * & 9.2 \\ \mathrm{E}^{-} & 1 & 1 & 2559405.6 & 2.1 & \mathrm{E}^{+} & 1 & 1 & 2630127.2 & -0.4 \\ \mathrm{E}^{+} & 2 & 2 & 2559316.7 & -0.4 & \mathrm{E}^{-} & 2 & 2 & 2630325.9 & -1.0 \\ \mathrm{E}^{-} & 3 & 3 & 2559183.1 & -3.8 & \mathrm{E}^{+} & 3 & 3 & 2630623.3 & 0.2 \\ \mathrm{E}^{+} & 4 & 4 & 2559005.4 & -6.4 & \mathrm{E}^{-} & 4 & 4 & 2631018.8 & 0.4 \\ \mathrm{E}^{-} & 5 & 5 & 2558783.3 & -7.3 & \mathrm{E}^{+} & 5 & 5 & 2631511.5 & 0.0 \\ \mathrm{E}^{+} & 6 & 6 & 2558517.4 & -4.5 & \mathrm{E}^{-} & 6 & 6 & 2632100.6 & 1.1 \\ \mathrm{E}^{-} & 7 & 7 & 2558212.0 & 7.9 & \mathrm{E}^{+} & 7 & 7 & 2632784.2 & -0.6 \\ \mathrm{E}^{+} & 8 & 8 & 2557837.0 & 1.8 & \mathrm{E}^{-} & 8 & 8 & 2633560.3 & 1.3 \\ \mathrm{E}^{+} & 1 & 0 & 2570321.3 & -2.0 & \mathrm{E}^{+} & 9 & 9 & 2634429.7 & -0.3 \\ \mathrm{E}^{-} & 2 & 1 & 2581209.1 & -2.2 & \mathrm{E}^{-} & 10 & 10 & 2635387.7 & -1.2 \\ \mathrm{E}^{+} & 3 & 2 & 2592107.4 & -1.7 & \mathrm{E}^{-} & 1 & 0 & 2637292.5 * & -15.9 \\ \mathrm{E}^{-} & 4 & 3 & 2603013.2 & -1.5 & \mathrm{E}^{+} & 2 & 1 & 2647878.9 * & -41.0 \\ \mathrm{E}^{+} & 5 & 4 & 2613928.2 & 2.1 & \mathrm{E}^{-} & 3 & 2 & 2658322.5 * & -61.0 \\ \mathrm{E}^{-} & 6 & 5 & 2624842.2 & 1.3 & \mathrm{E}^{+} & 4 & 3 & 2668620.8 * & -60.4 \\ \mathrm{E}^{+} & 7 & 6 & 2635755.6 & -0.6 & \mathrm{E}^{-} & 5 & 4 & 2678769.0 * & -31.0 \\ \mathrm{E}^{-} & 8 & 7 & 2646663.8 & -5.8 & \mathrm{E}^{+} & 6 & 5 & 2688753.4 * & 11.5 \\ & & & & & \mathrm{E}^{-} & 7 & 6 & 2698576.2 * & 40.4 \\ & & & & & \mathrm{E}^{+} & 8 & 7 & 2708235.1 * & -16.6\end{array}$

$\mathrm{K}_{\mathrm{a}}=0 \rightarrow 1$ Upper Bands

\begin{tabular}{|c|c|c|c|c|c|c|c|c|c|}
\hline$\Gamma i$ & $\overline{J^{\prime}}$ & $\mathrm{J}^{\prime \prime}$ & Frequency & $\mathrm{O}-\mathrm{C}$ & $\Gamma \mathrm{i}$ & $\overline{\mathrm{J}^{\prime}}$ & $\mathrm{J}^{\prime \prime}$ & Frequency & $\mathrm{O}-\mathrm{C}$ \\
\hline $\mathrm{A}_{1}^{+}$ & 7 & 8 & 2479547.6 & -1.6 & $\mathrm{~A}_{2}^{-}$ & 1 & 2 & $2607822.2^{*}$ & -22.1 \\
\hline $\mathrm{B}_{1}^{-}$ & 6 & 7 & 2490381.7 & 0.7 & $\mathrm{~B}_{2}{ }^{+}$ & 2 & 3 & $2596690.5^{*}$ & 6.8 \\
\hline $\mathrm{A}_{1}^{+}$ & 5 & 6 & 2501218.0 & 5.2 & $\mathrm{~A}_{2}^{-}$ & 3 & 4 & $2585379.3^{*}$ & 6.4 \\
\hline $\mathrm{B}_{1}^{-}$ & 4 & 5 & 2512057.6 & 4.5 & $\mathrm{~B}_{2}^{+}$ & 4 & 5 & $2573907.7 *$ & -2.8 \\
\hline $\mathrm{A}_{1}^{+}$ & 3 & 4 & 2522905.3 & 4.7 & $\mathrm{~A}_{2}^{-}$ & 5 & 6 & $2562288.8 *$ & -2.8 \\
\hline $\mathrm{B}_{1}^{-}$ & 2 & 3 & 2533753.0 & -1.0 & $\mathrm{~B}_{2}^{+}$ & 6 & 7 & $2550508.3 *$ & -2.3 \\
\hline $\mathrm{A}_{1}^{+}$ & 1 & 2 & 2544608.3 & -4.1 & $\mathrm{~A}_{2}^{-}$ & 7 & 8 & $2538563.5^{*}$ & 0.2 \\
\hline $\mathrm{B}_{1}^{-}$ & 1 & 1 & 2566280.9 & -5.4 & $\mathrm{~B}_{2}^{+}$ & 1 & 1 & 2637192.9 & 0.2 \\
\hline $\mathrm{A}_{1}^{+}$ & 2 & 2 & 2566178.0 & -1.8 & $\mathrm{~A}_{2}^{-}$ & 2 & 2 & 2637386.2 & 0.4 \\
\hline $\mathrm{B}_{1}^{-}$ & 3 & 3 & 2566018.0 & -2.3 & $\mathrm{~B}_{2}{ }^{+}$ & 3 & 3 & 2637675.8 & 0.2 \\
\hline $\mathrm{A}_{1}^{+}$ & 4 & 4 & 2565806.1 & -1.9 & $\mathrm{~A}_{2}^{-}$ & 4 & 4 & 2638060.8 & -0.3 \\
\hline $\mathrm{B}_{1^{-}}$ & 5 & 5 & 2565542.1 & -1.3 & $\mathrm{~B}_{2}{ }^{+}$ & 5 & 5 & 2638538.8 & -0.1 \\
\hline $\mathrm{A}_{1}^{+}$ & 6 & 6 & 2565226.6 & -0.3 & $\mathrm{~B}_{2}^{+}$ & 2 & 1 & $2651013.0 *$ & 9.5 \\
\hline $\mathrm{B}_{1}^{-}$ & 7 & 7 & 2564859.2 & 0.1 & $\mathrm{~A}_{2}^{-}$ & 3 & 2 & $2661421.5^{*}$ & 5.8 \\
\hline $\mathrm{A}_{1}^{+}$ & 8 & 8 & 2564442.2 & 1.8 & $\mathrm{~B}_{2}^{+}$ & 4 & 3 & $2671673.8 *$ & 4.3 \\
\hline $\mathrm{B}_{1}$ & 9 & 9 & 2563970.9 & -0.8 & $\mathrm{~A}_{2}^{-}$ & 5 & 4 & $2681759.9 *$ & 0.3 \\
\hline $\mathrm{A}_{1}{ }^{+}$ & 10 & 10 & 2563449.9 & -3.9 & $\mathrm{~B}_{2}^{+}$ & 6 & 5 & $2691674.4^{*}$ & -4.3 \\
\hline $\mathrm{B}_{1}^{-}$ & 11 & 11 & 2562891.6 & 3.8 & $\mathrm{~A}_{2}^{-}$ & 7 & 6 & $2701417.2^{*}$ & -2.8 \\
\hline
\end{tabular}




$\begin{array}{llllllllll}\mathrm{A}_{1}^{+} & 1 & 0 & 2577205.1 & -1.4 & \mathrm{~B}_{2}{ }^{+} & 8 & 7 & 2710978.1^{*} & 3.7 \\ \mathrm{~B}_{1}{ }^{-} & 2 & 1 & 2588074.6 & 0.0 & & & & \\ \mathrm{~A}_{1}{ }^{+} & 3 & 2 & 2598946.6 & 3.3 & & & & \\ \mathrm{~B}_{1}^{-} & 4 & 3 & 2609817.5 & 5.7 & & & & \\ \mathrm{~A}_{1}{ }^{+} & 5 & 4 & 2620686.3 & 6.6 & & & & \\ \mathrm{~B}_{1}^{-} & 6 & 5 & 2631545.9 & -0.5 & & & & \end{array}$

* The $\mathrm{P}$ and $\mathrm{R}$ branch lines for the three $\mathrm{K}_{\mathrm{a}}=0->1$ subbands labeled by " 2 " were not included in the fitted data set. The residuals for these transitions are representative of fitting only the P and R branches of each subband separately, as is described in Section 3.3.

acceptor tunneling motion relative to its value in $\mathrm{K}_{\mathrm{a}}{ }^{\prime \prime}=0$. A notational simplification is now introduced in which the $A_{1}+/-/ E^{+/-/ B} B_{1}^{+/-}$symmetry labels corresponding to the associated interchange triplet will be designated with a " 1 ", and the triplet defined by the $\mathrm{A}_{2}-1+/ \mathrm{E}^{-1+} / \mathrm{B}_{2}{ }^{-1+}$ species will be referred to with the label " 2 ". Hence, the energy difference between VRT components labeled " 1 " and " 2 " is the acceptor tunneling splitting. This is done so that the complicated notation of the symmetry labels can be avoided, and this labeling scheme for the observed VRT data will be used throughout. Also, the notation of upper, $E$, and lower is used to designate each VRT subband within a particular interchange triplet. It should be stressed that these labels refer only to the absorption frequencies of each transition between interchange triplets having acceptor tunneling labels corresponding to "1" or "2", for which upper and lower define the highest and lowest frequency VRT subband (relative to the fitted band origin, $v_{0}$ ) and $E$ represents the center subband. This notation is necessary because the A and B symmetry species alternate between the higher $(+)$ and lower (-) energy components of the interchange triplet with alternating J. These definitions are also illustrated in Figure 3.2. Interchange tunneling is fit by including an energy shift in the fitted band origins which reproduce the $A / E / B$ splitting in each $|v, J, K\rangle$, as is qualitatively shown in Figure 3.2. This energy term takes the form $v_{0}+1-\left(E_{D A T T}\right) / 2$, for which $v_{0}$ is the fitted band origin as described above, and EDAIT is the fitted energy of 
the Donor Acceptor Interchange Iunneling splitting between the VRT components of A/B symmetry. The sum in this expression corresponds to the $(+)$ energy component and the difference represents the (-) energy component. A similar energy expression of the form $v_{0}$ $+E_{D T}$ is used to fit the effect of Donor Tunneling, for which $E_{D T}$ is the energy deviation which shifts the $\mathrm{E}$ band away from the fitted band origin. Finally, a plot of all 12 VRT bands is shown in Figure 3.4 and this spectrum will be referenced throughout this manuscript when discussing a particular aspect of this work. In this figure the intensities are calculated by assuming a fitted $4.7 \mathrm{~K}$ rotational temperature for all the data, using the appropriate Honl-London factors for $\Delta \mathrm{J}=0,+/-1, \Delta \mathrm{K}=0,+1$ transitions (where $\Delta \mathrm{J} \neq 0$ for $\Delta \mathrm{K}=0$ ), and employing the nuclear spin weights corresponding to the assigned symmetry species for each observed VRT transition.

\subsection{3 $K_{a}=0->0 V R T$ Subbands}

Only two of the possible six $\mathrm{K}_{\mathrm{a}}=0 \rightarrow 0$ VRT subbands were previously observed in Reference 10, and because of this, it was assumed that the acceptor tunneling splitting was large in the excited vibrational state. With the added sensitivity recently obtained in this spectral region, all six $K_{a}=0 \rightarrow 0$ VRT subbands have now been observed and are reported in Table 3.1. The fitted rotational constants for each band are reported in Table 3.2 , such that the rotational constants with the labels upper, E, and lower refer to the constants of the upper, E, and lower VRT subbands. The six tunneling subbands are arranged into two groups of three according to the similarity of the excited state rotational constants. The average values for the two sets of excited state rotational constants are $5395.699(66) \mathrm{MHz}$ and $5407.212(68) \mathrm{MHz}$, as can be verified from Table 3.2. This grouping separates the VRT transitions between the two distinct interchange triplets possessing symmetries of " 1 " and " 2 " for the $K_{a}=0-0 \mathrm{a}$ - type subbands. These two fitted band origins of $2477298.9(10)$ and $2477618.98(90) \mathrm{MHz}$ are used to distinguish between the "1" and "2" triplets. The band origins are recognized as the gap in the 2477 
Figure 3.4 Caption

The entire high resolution VRT spectrum of the intermolecular vibration of the water dimer $\mathrm{d}_{4}$ is presented. The arrows are included to emphasize specific features of the spectrum. The vertical arrows identify the four fitted band origins described in the text. Arrow (a) in the $2477 \mathrm{GHz}$ spectral region signifies the two near resonant fitted band origins for the $\mathrm{K}_{\mathrm{a}}$ $=0 \rightarrow 0$ transitions, whereas the vertical arrows near 2560 (b) and 2630 (c) $\mathrm{GHz}$ correspond to the two $K_{a}=0->1$ VRT bands represented by VRT states of " 1 " and " 2 " symmetries, respectively. The horizontal arrow (d) positioned in the $\mathrm{P}$ branch of the $\mathrm{K}_{\mathrm{a}}=$ $0 \rightarrow 0$ bands near $2455 \mathrm{GHz}$ represents the sum (ca. $4-5 \mathrm{GHz}$ ) of the ground and excited states donor acceptor interchange tunneling splitting, and the horizontal arrows in the 2560 (e) and 2630 (f) GHz spectral regions identify the sum (ca. 13-14 GHz) of the two donoracceptor interchange splittings in $Q$ branches of the $K_{a}=0 \rightarrow 1$ bands. The separation of $70 \mathrm{GHz}$ between the two sets of tripled $\mathrm{Q}$ branches is the change in the acceptor tunneling splitting in $\mathrm{K}_{\mathrm{a}}{ }^{\prime}=1$, relative to $\mathrm{K}_{\mathrm{a}}{ }^{\prime \prime}=0$. The relative nuclear spin weight for each ground state VRT level is used along with the appropriate Honl-London factors and a fitted temperature of $4.7 \mathrm{~K}$ to calculate the intensities. 
Figure 3.4

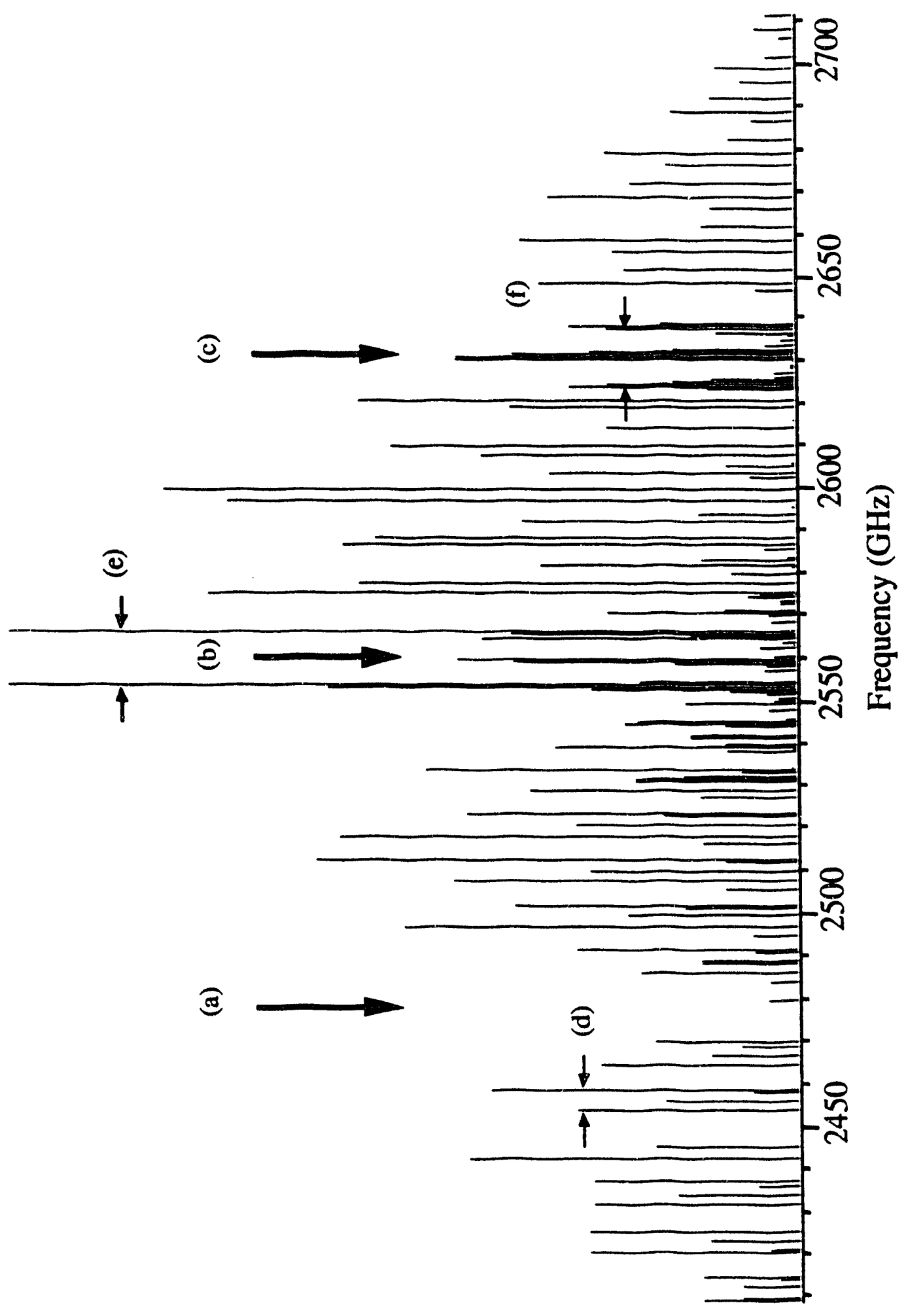


Table 3.2

Fitted rotational constants for the ground and excited vibrational states (in $\mathrm{MHz}$ ). All of the rotational constants for the ground state are fixed at the reported values of Reference 24 and the excited state data were fit as is described in Section 3.3. Parameters not included in the table have been fixed to a value of zero.

Ground State $\mathrm{K}_{\mathrm{a}}=0$

\begin{tabular}{|c|c|c|c|c|}
\hline \multicolumn{5}{|c|}{ "1" } \\
\hline $\begin{array}{l}\text { Blower } \\
B_{\text {upper }} \\
\text { Dlower/upper } \\
\end{array}$ & $\begin{array}{l}5432.5724(29) \\
5432.1342(28) \\
0.036429(44) \\
\end{array}$ & $\begin{array}{l}\mathrm{BE}_{\mathrm{E}} \\
\mathrm{DE} \\
\mathrm{HE} \\
\end{array}$ & $\begin{array}{l}5432.3290(12) \\
0.03558(24) \\
-0.00001130(13) \\
\end{array}$ & $\begin{array}{l}\text { Blower } \\
\text { Bupper } \\
\text { Dlower/4 }\end{array}$ \\
\hline \multicolumn{5}{|c|}{$\mathrm{K}_{\mathrm{a}}=0->0$ Bands } \\
\hline & "1" & \multicolumn{3}{|c|}{$" 2 "$} \\
\hline$v_{0}$ & $2477298.9(10)$ & \multirow[b]{2}{*}{$\begin{array}{l}\text { Vo } \\
\text { Blower }_{\text {low }} \\
B_{E} \\
B_{\text {upper }} \\
\text { Dlower } \\
D_{E} \\
D_{\text {upper }}\end{array}$} & \multicolumn{2}{|c|}{$2477618.98(90)$} \\
\hline $\begin{array}{l}\text { B } \\
B_{E} \\
B_{\text {upper }} \\
D_{E} \\
\text { Dlower/upper }\end{array}$ & $\begin{array}{l}5406.851(62) \\
5406.974(80) \\
5407.811(61) \\
0.03891(77) \\
0.03604(56)\end{array}$ & & \multicolumn{2}{|c|}{$\begin{array}{l}5393.127(80) \\
5395.783(82) \\
5398.187(38) \\
0.00605(77) \\
0.01135(78) \\
0.01653(20)\end{array}$} \\
\hline
\end{tabular}

Value for $\mathrm{A}^{\prime}$ rotational constant ca. $122.9 \mathrm{GHz}_{\text {. }}^{\text {(a) }}$

$$
\mathrm{K}_{\mathrm{a}}=0 \rightarrow 1 \text { Bands }
$$

\begin{tabular}{|c|c|c|c|}
\hline & $" 1 "$ & & "2"(b) \\
\hline$v_{0}$ & $2559779.71(89)$ & $v_{0}$ & $2630079.7(20)$ \\
\hline $\begin{array}{l}\text { B lower } \\
B_{E} \\
B_{\text {upper }} \\
\text { (B-C)lower } \\
\text { (B-C)E } \\
\text { (B-C) upper } \\
\text { Dlower } \\
D_{E} \\
D_{\text {upper }}\end{array}$ & $\begin{array}{l}5427.248(92) \\
5424.70(10) \\
5419.732(57) \\
59.33(13) \\
55.344(75) \\
55.242(86) \\
0.0328(12) \\
0.0491(14) \\
0.03264(53)\end{array}$ & $\begin{array}{l}\text { B lower } \\
B_{E} \\
B_{\text {upper }} \\
D_{\text {lower }} \\
D_{E} \\
\text { Dupper }\end{array}$ & $\begin{array}{l}5483.172(79) \\
5482.13(10) \\
5481.02(55) \\
0.04337(60) \\
0.04654(90) \\
0.048(16)\end{array}$ \\
\hline
\end{tabular}

Standard Deviation of Fit $=2.62 \mathrm{MHz}$

(a) $A^{\prime}$ is not a fitted parameter.

(b) The rotational constants representing the results of three separate fits for the $P$ and $R$ branch transitions only, for each of the three subbands of " 2 " symmetry are Blower $=$ 5367.72(16), $\mathrm{D}_{\text {lower }}=0.0711(21), \mathrm{B}_{\mathrm{E}}=5372.30(33), \mathrm{DE}_{\mathrm{E}}=0.4635(11), \mathrm{H}_{\mathrm{E}}=$ $0.00330(10), B_{\text {upper }}=5358.27(14)$, and $D_{\text {upper }}=0.0832(20)$. 
$\mathrm{GHz}$ spectral region of Figure 3.4, and are designated with the marker (a). Upon close inspection of these subbands, two sets of evenly spaced triplets are noticed for each $\mathrm{P}$ and $\mathrm{R}$ branch transition. The spacing of these triplets correspond to the change in the donoracceptor interchange tunneling splitting relative to the ground state value, for the acceptor tunneling levels " 1 " and " 2 ". The marker labeled (d) in Figure 3.4, indicates that the spacing of each set of triplets is nearly the same, verifying that the two observed excited state interchange splittings are similar. The similarity of the band origins is also verified since the centers of the two sets of $\mathrm{P}$ and $\mathrm{R}$ branch triplets occur at approximately the same frequency. Since the band origins are nearly identical, the VRT levels of "1" symmetry must reside on the same side of the acceptor tunneling splitting with respect to the ground state. If they were on different sides, it would not be possible for the two band origins to have such similar values. These results conclusively show that when comparing the $\mathrm{K}_{\mathrm{a}}=0$ manifolds, the acceptor tunneling splitting does not appreciably change relative to its value in the ground state, contrary to the assumption made in the Reference 10.

From the small variation of the acceptor tunneling splitting, it is deduced that the VRT levels characterized by the " 1 " symmetry labels reside on the bottom half of the acceptor tunneling splitting in the excited state, as is the case for $v=0$. Yet the ordering of the symmetry species within each excited state interchange triplet cannot be determined by simply fitting these band origins. Several configurations could exist when considering that the upper or lower band could originate from either the $(+)$ or $(-)$ side of each ground state interchange triplet. Each possibility would result in a very different value of $E_{D A r T}$ in the excited state. Therefore, it is important to assign unambiguous symmetry labels to each VRT state in the vibrational manifold. Unfortunately, combination differences are not useful in assigning ground state symmetries species for each transition, because the rotational constants from the microwave data ${ }^{24}$ are identical to within the precision of the FIR spectrometer. Therefore, to obtain this information, it is necessary to consider the unique relative nuclear spin weights for each symmetry species. Transitions within the 
upper and lower bands of each interchange splitting will give rise to intensity alternations with $\mathrm{J}$, as is indicated in Equation 3.1 and the discussion which follows, and these alternations may be examined to confirm a symmetry assignment for a particular VRT transition.

In Figure 3.5 the $\mathrm{R}(2)$ transitions of each lower band associated with the two distinct interchange triplets are shown. These two transitions at frequencies of 2507194.0 and $2507368.4 \mathrm{MHz}$ occur so close together that similar spectrometer conditions are found to exist across this scan. When scaling for the slight variation in laser power at the two discrete transition frequencies, the relative intensity ratio is determined to be ca. 2.8. This indicates that these transitions should be assigned as originating from VRT states having $B_{1}$ and $B_{2}$ symmetries, respectively, since inspection of Equation 3.1 provides an actual ratio of 2.5 for these symmetry species. Since the two $R(2)$ transitions belong to the lower bands and since the ordering of the ground state VRT symmetries is known, these transitions must originate from the $(+)$ component and terminate at the $(-)$ component of their respective ground and excited state interchange triplets. Therefore, the donor-acceptor interchange tunneling parameters have been fit so that the transitions connect different sides of the interchange triplets; i.e, $(+) \rightarrow(-)$ and $(-) \rightarrow(+)$. From this result, the three bands yielding $\mathrm{B}^{\prime}$ values of ca. $5407 \mathrm{MHz}$ were grouped together as transitions between states of "1" symmetry, while the three bands with B' values of ca. $5395 \mathrm{MHz}$ are assigned with the "2" symmetry labels. The change in donor tunneling is then represented for these VRT subbands as the deviation of the $m^{\text {iddle }} \mathrm{E}$ band from the fitted vibrational band origins $\left(v_{0}\right)$. The values of all tunneling parameters for $K_{a}=0$ in both the ground and vibrationally excited state are included in Table 3.3 and will be discussed in Secrion 3.4 .

With the symmetry assignments reasonably well established, the rotational constants for the six VRT subbands may be examined. In doing so, it should be noted that the deuterium atoms provide a minor contribution to $B$, because this rotational constant is essentially invariant throughout a particular VRT motion due to the two heavy oxygen 
Figure 3.5

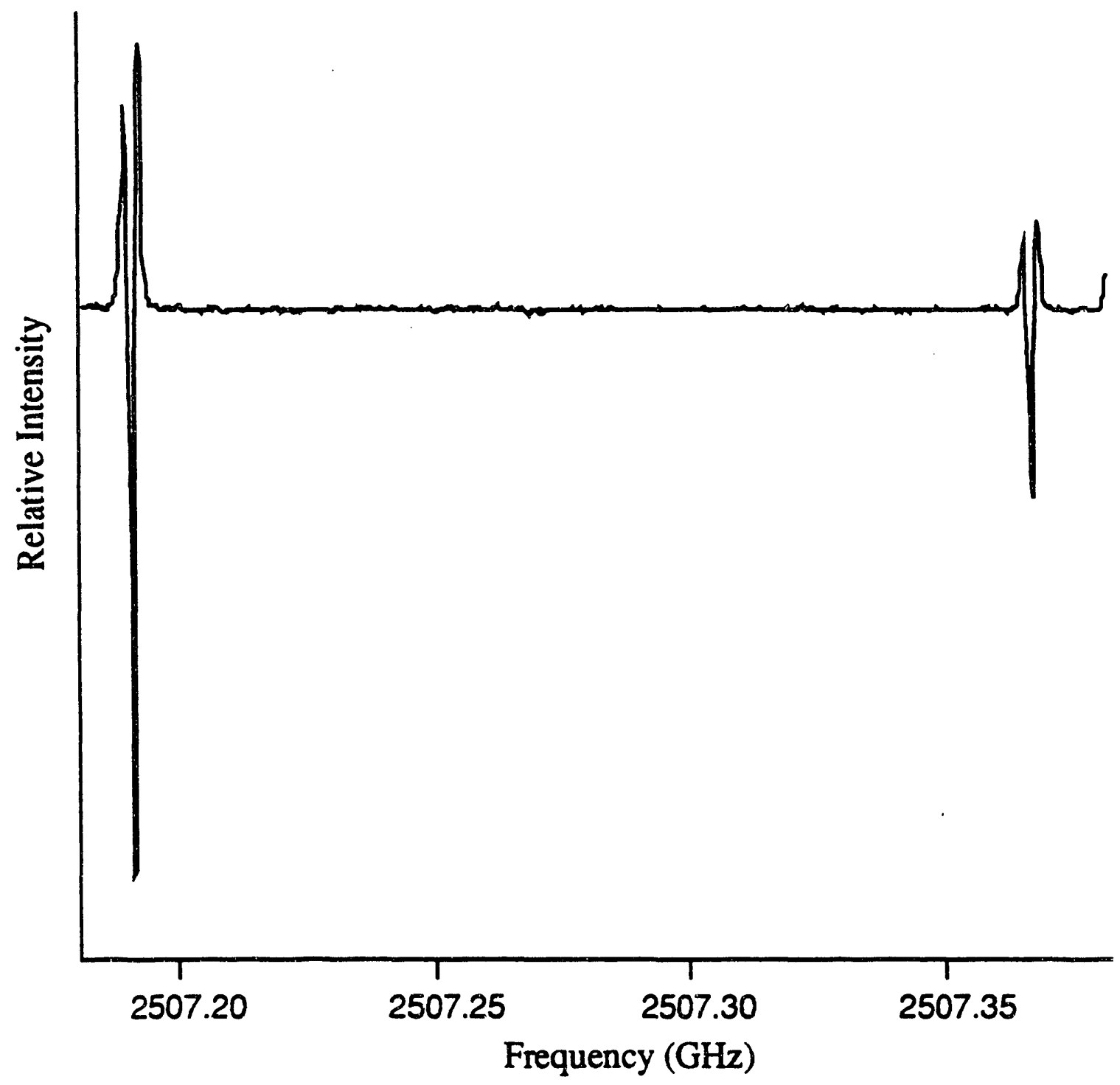

This $200 \mathrm{MHz}$ scan illustrates the intensity ratio of the $\mathbf{R}(2)$ transitions for each of the lower $\mathrm{K}_{\mathrm{a}}=0 \rightarrow 0$ VRT bands at 2507194 and $2507368 \mathrm{GHz}$. The ground state ordering of the symmetry species is known, and this allows for the intensity ratio to be used in determining the ordering of the symmetries species in the excited vibrational state. Since both transitions belong to the lower bands and their intensity ratio is best described by ground state VRT symmetries of $B_{1}$ and $B_{2}$, they must both originate from the $(+)$ side of the ground state donor acceptor interchange triplet. This assignment verifies that transitions between the interchange triplets are made from $(+) \rightarrow(-)$ and $(-) \rightarrow(+)$. It also indicates that the acceptor tunneling splitting in $K_{a}{ }^{\prime}=0$ is nearly identical to that of $K_{-a}{ }^{\prime \prime}=0$, as described in the text. 
Table 3.3

The fitted tunneling splittings for the ground and vibrationally excited states (in $\mathrm{MHz}$ ). The ground state values were fixed at the values reported in Reference 24.

\section{Ground State Tunneling Splittings}

\begin{tabular}{|c|c|c|c|}
\hline Donor-Acceptor Interchange & "l" & $\mathrm{K}_{\mathrm{a}} "=0$ & 1172.115 \\
\hline Donor-Acceptor Interchange & " $2 "$ & $\mathrm{~K}_{\mathrm{a}}{ }^{\prime \prime}=0$ & 1083.303 \\
\hline Donor-Acceptor Interchange & $" 1 "$ & $\mathrm{~K}_{\mathrm{a}}{ }^{\prime \prime}=1$ & 1076.616 \\
\hline Donor-Acceptor Interchange & "2" & $\mathrm{K}_{\mathrm{a}} "=1$ & 992.368 \\
\hline $\begin{array}{l}\text { Change in Donor Tunneling } \\
\text { Between } \mathrm{K}_{\mathrm{a}}{ }^{\prime}=0 \text { and } 1\end{array}$ & “1" & & 14.0 \\
\hline $\begin{array}{l}\text { Change in Donor Tunneling } \\
\text { Between } \mathrm{K}_{\mathrm{a}}{ }^{\prime}=0 \text { and } 1\end{array}$ & "2" & & 13.4 \\
\hline Donor-Acceptor Interchange & “1” & $\mathrm{K}_{\mathrm{a}}{ }^{\prime \prime}=2$ & 808.72 \\
\hline $\begin{array}{l}\text { Change in Donor Tunneling } \\
\text { Between } \mathrm{K}_{\mathrm{a}}=1 \text { and } 2\end{array}$ & $" 1 "$ & & 12.840 \\
\hline
\end{tabular}

Excited Vibrational State Tunneling Splittings

Change in Acceptor Tunneling Splitting for $\mathrm{K}_{\mathrm{a}}{ }^{\prime}=0$ (a) 320.08 (95)

Donor-Acceptor Interchange “ 1 " $\quad \mathrm{K}_{\mathrm{a}}{ }^{\prime}=0 \quad 3348.6(16)$

Change in Donor Tunneling(b) “ 1 " $\quad \mathrm{K}_{\mathrm{a}}{ }^{\prime}=0 \quad 154.0(17)$

Donor-Acceptor Interchange “ 2 " $\quad \mathrm{K}_{\mathrm{a}}{ }^{\prime}=0 \quad 3926.1(18)$

Change in Donor Tunneling(b) ' 2 " $\quad \mathrm{K}_{\mathrm{a}}{ }^{\prime}=0 \quad 40.8(17)$

Change in Accepwr Tunneling Splitting for $\mathrm{K}_{\mathrm{a}}{ }^{\prime}=1^{(\mathrm{a})} 70300.0(15)$

Donor-Acceptor Interchange “ 1 " $\quad \mathrm{K}_{\mathrm{a}}{ }^{\prime}=1 \quad 11947.7(18)$

Change in Donor Tunneling(b) “ 1 ” $\quad \mathrm{K}_{\mathrm{a}}{ }^{\prime}=1 \quad-333.3(16)$

Donor-Acceptor Interchange ' 2 " $\quad \mathrm{K}_{\mathrm{a}}{ }^{\prime}=1 \quad 12948.9$ (39)

Change in Donor Tunneling(b) ' 2 " $\quad \mathrm{K}_{\mathrm{a}}{ }^{\prime}=1 \quad-52.4 \quad$ (28)

(a) Relative to $\mathrm{K}_{\mathrm{a}}{ }^{\prime \prime}=0$.

(b) Relative to $\mathrm{K}_{2} "=0$ for the particular interchange triplet. 
atoms. It is found that within the above two sets of bands, the B' values vary on average by only $-0.7 \%$ and $-0.5 \%$ relative to the $\mathrm{K}_{\mathbf{a}}{ }^{\prime \prime}=0$ constants. Surely these small changes reflect inertial stability, yet the negative deviation is consistent with the 3-4 fold increase in the tunneling splittings found to exist in the interchange coordinate of the excited state motion because this tunneling motion produces a vibrationally averaged structure in which the $\mathrm{H}$-bonded deuterium atom is displaced away from the O-D $\cdots O \mathrm{O} \mathrm{A}^{\prime}$ inertial axis. Also, the distortion constants for the three VRT subbands of the interchange triplet associated with the " 2 " symmetry label are nearly a factor of three smaller than for the subbands of " 1 " symmetry, in which the $D^{\prime}$ values are very similar to those of $v=0, K_{a}{ }^{\prime \prime}=0$. The similarity of these constants within a given set of VRT subbands further supports the above method of separating the $\mathrm{K}_{\mathrm{a}}=0 \rightarrow 0$ transitions.

\subsection{4 $K_{a}=0->1$ VRT Subbands}

In the preliminary work of Reference 10 , only half of the $K_{a}=0 \rightarrow 1$ VRT bands were observed. From the data of $\mathrm{Zwart}$ et al. ${ }^{7}$ a ground state structural $\mathrm{A}_{s}$ " rotational constant can be determined to be $125.7 \mathrm{GHz}$. With the present knowledge of the six $\mathrm{K}_{\mathrm{a}}=$ $0->0$ VRT subbands, a frequency estimate for the remaining three $K_{a}=0->1$ subbands can be calculated as,

$$
\begin{gathered}
\left.A_{S^{\prime}}+B+\left(V O \alpha^{\prime \prime} 1^{n} ; K^{\prime}=0\right)+V_{O}\left({ }^{\prime \prime} 2^{n} ; K^{\prime}=0\right)\right) / 2=2597.7 \mathrm{GHz} \\
2(2597.7 \mathrm{GHz})-v_{0}\left({ }^{\prime \prime} 1^{n} ; K^{\prime}=1\right)=2635.7 \mathrm{GHz},
\end{gathered}
$$

in which Equation (3.3) assumes the structural (non-tunneling) value for $A_{s}$ ' leading to a $\mathrm{K}_{\mathrm{a}}=0 \rightarrow 1$ rovibrational transition occurring at ca. $2597.7 \mathrm{GHz}$. Equation (3.4) uses this result from which the large acceptor tunneling splitting is taken into account to predict the frequency of the three previously unidentified $\mathrm{K}_{\mathrm{a}}=0 \rightarrow 1 \mathrm{VRT}$ bands at $2635.7 \mathrm{GHz}$. These three VRT bands have now been located in this spectral region and the transitions are included in Table 3.1. 
The three VRT bands previously measured near $2560 \mathrm{GHz}$ have been assigned as originating from the states having " 1 " symmetry. Furthermore, the three bands were fit so that the transitions occurred as $(+)->(+)$ and $(-) \rightarrow(-)$ between the ground and excited state interchange triplets. To confirm this assignment, the calibration of $\mathrm{P}$ and $\mathrm{R}$ branch lines to a precision of $400 \mathrm{kHz}$ was conducted for the lower and upper subbands centered about the $2559779.71(88) \mathrm{MHz}$ fitted band origin. Ground state combination differences were fit, and this established that the transitions actually should have been fit as $(+)->(-)$ and $(-) \rightarrow(+)$, as was found to be the case in the $\mathrm{K}_{\mathrm{a}}=0 \rightarrow 0$ subbands.

Unfortunately, the precision with which the calibrated transitions were measured could not distinguish between the rotational constants of states having " 1 " or " 2 " symmetries. To establish this aspect of the assignment, relative intensities of the individual $Q$ branch transitions were relied upon, as in the earlier work ${ }^{10}$. However, instead of simply measuring peak heights, enhanced sensitivity allowed for actual fractional absorption measurements to be carried out on the upper and lower $Q$ branches located near $2560 \mathrm{GHz}$. In these experiments, the Fabry-Perot etalon was used to isolate only the lower sideband when scanning with the $2588361.8 \mathrm{MHz}\left({ }^{13} \mathrm{CH}_{3} \mathrm{OH}\right)$ laser line, from which both $\mathrm{Q}$ branches could be detected. Each $\Delta \mathrm{J}=0$ absorption was measured with the lock-in amplifier and the signal was scaled to the amount of AM single sideband power focussed onto the detector at the exact $(+/-1 \mathrm{MHz})$ frequency of the absorption. With this approach, laser power fluctuations over the $100 \mathrm{MHz}$ scan were irrelevant and the alternation in intensity of the transitions with $\mathrm{J}$ was determined to accurately reflect the ordering of the excited state symmetries. The data were then fit to a rotational temperature of $4.7 \mathrm{~K}(+/$ 0.2 ) and simulations of the $Q$ branches at this temperature for all possible configurations of transitions between the $(+)$ and $(-)$ components of the interchange triplets were compared to. the observed spectral intensities. Figure 3.6 shows the upper and lower $Q$ branches of the $2560 \mathrm{GHz}$ VRT bands for each of the four possible excited state interchange tunneling configurations labeled as $(A),(B),(C)$ and $(D)$, along with the absolute measured 
Figure 3.6
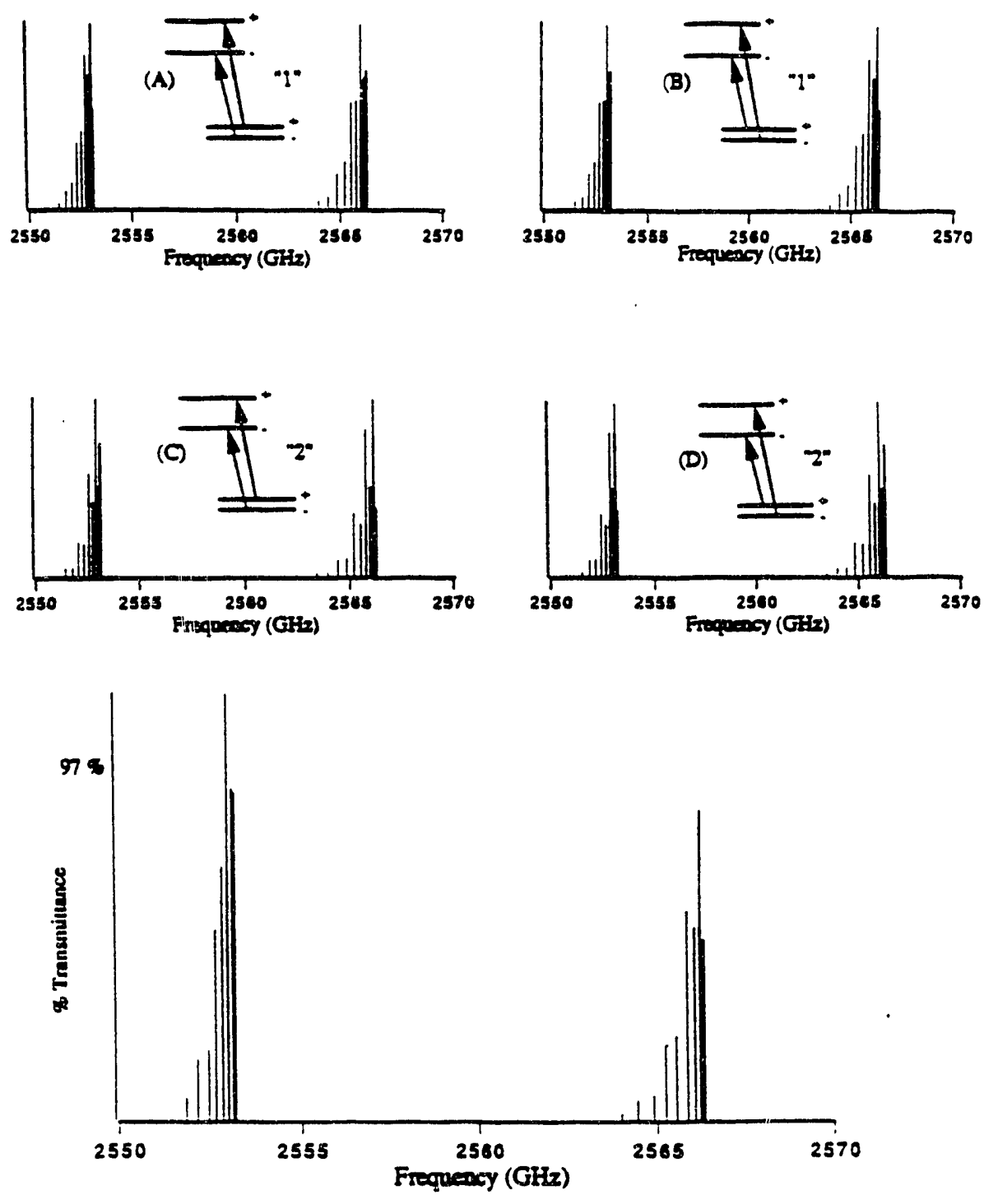

Each of the cases labeled (A)-(D) illustrate a possible configuration for the transitions of the $2560 \mathrm{GHz}$ VRT bands. The inserts of each case diagram the (+) and (-) energy levels about the donor acceptor interchange splitting for both the ground and vibrational states. The arrows represent transitions between these energy levels and the proper configuration depends on the ordering of the vibrational state symmetries. The " 1 " and " 2 " indicate that the transitions take place between the $A_{1}+/-/ E^{+1 /-/ B_{1}}+/=$ and $A_{2} /+/ E^{-1+/} / B_{2}^{-/+}$symmetry species, respectively. For each possibility, a different alternation of intensities within the upper and lower band $Q$ branches results, and each of these possibilities are simulated at 4.7 K. The bottom spectrum is the result of the fractional absorption measurements carried out on the $2560 \mathrm{GHz}$ upper and lower $\mathrm{Q}$ branches. The intensity alternation is represented best by the simulation in (b), thus these states were fit with the " 1 " ground state VRT symmetries, and the excited state symmetries were ordered so that the $(+) \rightarrow(-)$ and $(-) \rightarrow$ $(+)$ configuration resulted as was found for the $K_{a}=0->0$ bands. 
intensities (in \% transmittance) of the $Q$ branches. The inserts within each of the calculated spectra indicate the type of transition between the (+) and (-) components for the each possible case. The peak absorbance for the upper $Q$ branch near $2566 \mathrm{GHz}$, is weaker than the lower $Q$ branch, because of the limited modulation depth $(\Delta f)$ of the microwave synthesizer in this spectral region, when scanning with the $2588 \mathrm{GHz}{ }^{13} \mathrm{CH}_{3} \mathrm{OH}$ laser. This is an experimental artifact of the spectrometer, and it does not affect the relative intensities within a $Q$ branch - only the peak intensity of the upper $Q$ branch versus that of the lower is affected. Clearly case (B) is reproduced most accurately by the experimental data, whereas the bands had been previously fit by applying the case (A) assignment. This result establishes that the transitions do originate from the VRT states of "1" symmetry, and cross the interchange triplet, as was determined with the calibrated combination difference data. It was then deduced that the bands located near $2630 \mathrm{GHz}$ were of "2" symmetry, and these interchange splittings were also fit as transitions originating from the (+) and terminating at the $(-)$ components. Absolute intensities were not measured for the $\mathrm{Q}$ branches in these bands because a number of strong atmospheric water transitions $(533<-$ $5_{24}$ at $2630961.2 \mathrm{MHz}$ and $4_{14}<-303$ at $2640473.4 \mathrm{MHz}$ ) which are heavily populated at room temperature, made scanning in this spectral region extremely difficult. Only under optimal purging conditions of the entire laser beam path with a steady flow of dry $\mathrm{N}_{2}$ could the transitions be observed at all. Finally, as in the case of the $K_{a}=0 \rightarrow 0$ bands, the effect of donor tunneling is fit as the deviation of the $\mathrm{E}$ band from the fitted band origin. All $\mathrm{K}_{\mathrm{a}}{ }^{\prime}$ $=1$ tunneling parameters are reported in Table 3.3 for the vibrationally excited state.

Since the symmetry assignment has been established for each of the six $\mathrm{K}_{\mathrm{a}}=0 \rightarrow 1$ VRT bands, the fitted rotational constants for these bands can be obtained, and are reported in Table 3.2. For the three bands near $2560 \mathrm{GHz}$, the constants vary slightly from those reported previously 10 and they reflect the larger data set. The three bands were fit as $c$ type rovibrational bands. The $\mathrm{B}^{\prime}$ rotationai constants are only slightly smaller than those of the $\mathrm{K}_{\mathrm{a}} "=0$ and $\mathrm{K}_{\mathrm{a}} "=1$ values, however the asymmetry parameters have increased by 
nearly a factor of two in the excited vibrational state. A simultaneous fit of all six $K_{a}=0->$ 0 bands and the three $\mathrm{K}_{\mathrm{a}}=0->1$ bands at 2559779.71 (88) $\mathrm{MHz}$ results in a standard deviation of $2.8 \mathrm{MHz}$, which is within the FIR-VRT spectrometer frequency uncertainty in this spectral region, and the residuals for these bands indicate that the fit is of high quality. The inclusion of the three $Q$ branches of the bands located near $2630 \mathrm{GHz}$ into the VRT fit yielded a standard deviation of $2.6 \mathrm{MHz}$, and the residuals for these transitions relate only to this fit. These three $\mathrm{Q}$ branches permit the determination of all tunneling parameters which are reported in Table 3.3, a vibrational band origin of 2630079.7(20) for the remaining three $\mathrm{K}_{\mathrm{a}}=0 \rightarrow 1$ subbands, and an average rotational constant of 5482.11(24) MHz. This B' value may not fully represent the rotational constants for these three subbands, because the $P$ and $R$ branch transitions have not been included in this fit. This fit illustrates that the interchange splitting associated with these bands is nearly the same as that measured for the $2560 \mathrm{GHz}$ VRT bands. All of the $\mathrm{P}$ and $\mathrm{R}$ branch transitions within the subbands of "2" symmetry are unambiguously assigned as water dimer transitions by confirming ground state combination differences, however, the residuals obtained (see Table 3.1) for these transitions when fitting the bands individually are very large and indicate that a set of perturbing VRT states is close in energy. A Coriolis type of perturbation is proposed as the cause of the large residuals. The selection rules for this type of perturbation allow for the interaction of VRT states between $\mathrm{K}_{\mathrm{a}}{ }^{\prime}$ manifolds which are off diagonal by $+/-1$, diagonal in the $J$ quantum number, and posses the same

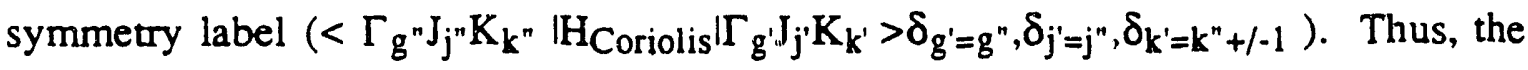
perturbation most probably arises from the $\mathrm{K}_{\mathrm{a}}{ }^{\prime}=0$ manifold of another intermolecular vibration at higher energy, because only one of the asymmetry components (related to the $P$ and $\mathrm{R}$ branch transitions) for the set of $2630 \mathrm{GHz} \mathrm{K}_{\mathrm{a}}=0 \rightarrow 1 \mathrm{VRT}$ bands shift to lower energy. This situation is qualitatively illustrated in Figure 3.7 by assuming a Coriolis perturbation. At present no other evidence for the next vibration has been observed. When fitting the $P$, and $R$ branches together with the entire data set, unrealistic values for $B$ and 
Figure 3.7

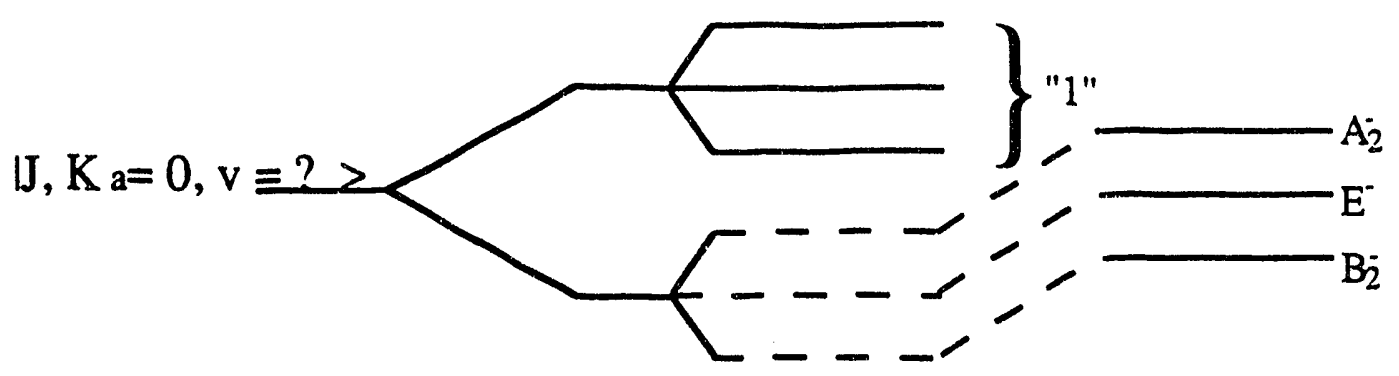

$K_{\mathrm{a}}=0$ Manifold of Next Vibration

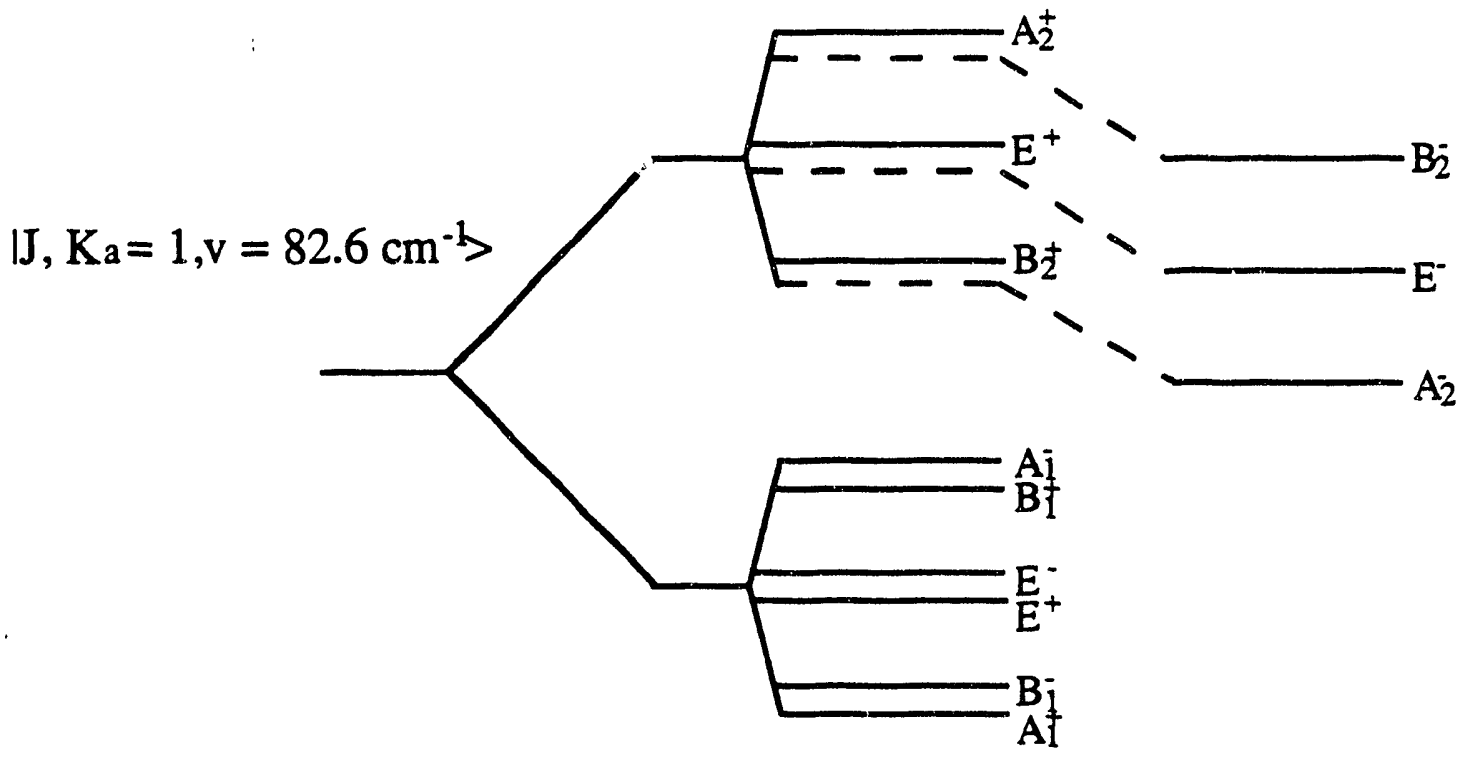

$\mathrm{K}_{\mathrm{a}}=1$ Manifold of $82.6 \mathrm{~cm}^{-1}$ Vibration

This energy level diagram shows how a $K_{a}=0$ manifold would perturb the $K_{a}=1$ VRT species having the " 2 " symmetry labels. The only other possible perturbing states would be associated with $K_{a}>0$ manifolds, however, such a situation would push both of the $K_{a}$ $=1$ asymmetry components of " 2 " symmetry to lower energy. Since only the lower asymmetry components of the observed $\mathrm{K}_{\mathrm{a}}=1$ VRT states ase being shifted, assuming that the band is of $c$-type, the perturbation must result from the $\mathrm{K}_{\mathrm{a}}=0$ manifold associated with the next intermolecular vibration at higher frequency. The $\mathrm{K}_{\mathrm{a}}=0$ states of " 2 " symmetry for the unobserved vibration, are depicted in an arbitrarily. 
(B - C) are obtained and the residuals of the fit were extremely large; thus, the results of this combined fit are not included here.

Finally, a value of the $\mathrm{A}^{\prime}$ rotational constant can be calculated by

$$
\left.\mathrm{A}^{\prime}=\left(v_{0}\left({ }^{n} 1^{n} ; \mathrm{K}^{\prime}=1\right)^{+}+v_{O(}{ }^{n} 2^{n} ; \mathrm{K}^{\prime}=1\right)\right) / 2-\left(v_{0\left({ }^{n} 1^{n} ; \mathrm{K}^{\prime}=0\right)}+v_{0\left(2^{n} ; \mathrm{K}^{\prime}=0,\right)}\right) / 2+\mathrm{B}^{\prime}=122.9 \mathrm{GHz},(3.5)
$$

in which an approximate value of $B^{\prime}=5.4 \mathrm{GHz}$ is used. Equation 3.5 simply takes the difference of the averaged $K_{a}=0 \rightarrow 1$ band origins and the averaged $K_{a}=0 \rightarrow 0$ band origins and appropriately accounts for the $\mathrm{B}^{\prime}$ rotational contribution in the $\mathrm{K}_{\mathrm{a}}{ }^{\prime}=1$ manifold to arrive at the value for $A^{\prime}$. The previous work made the assumption that very little $\mathrm{K}_{\mathrm{a}}{ }^{\prime}$ dependence of the acceptor tunneling existed and this resulted in a value of $\mathrm{A}^{\prime}=85 \mathrm{GHz}$ from the spectral evidence measured at that time. This assumption clearly led to an inaccurate value of this rotational constant, for it is readily apparent in the present work that acceptor tunneling is in fact largely influenced by $K_{a}^{\prime}$. It is useful to compare $A^{\prime}$ with the corresponding ground state value, because the deuterium atom tunneling motions should be reflected in the $A$ rotational constant. The value of $A$ " has been reported from the $K_{a}=1 \rightarrow$ $2, v=0$ microwave study of Zwart et al. ${ }^{7}$ to be $120.3 \mathrm{GHz}$, however this work seems to have neglected to account for the contribution of the $\mathrm{B}$ " rotational constant, and when doing so it is noticed that its value is actually $\mathrm{A}^{\prime \prime}=125.7 \mathrm{GHz}$. This corrected value agrees well with the most recent $v=0$ determination of $A^{\prime \prime}=124.9 \mathrm{GHz}$ determined from the $\mathrm{K}_{\mathrm{a}}=0$ $>1$ microwave experiments of Karyakin et al. ${ }^{8}$ The nominal $2 \%$ reduction in $\mathrm{A}^{\prime}$ relative to $\mathrm{A}^{\mathrm{N}}$ is indicative of the cluster accessing a vibrationally averaged structure for which the deuterium atoms spend more time away from the top axis, which is consistent with the larger tunneling splittings exhibited in the excited vibrational manifold. 


\subsection{The Tunneling Splittings}

\subsubsection{An IAM Overview}

This discussion begins with a brief review of the generalized IAM model used to analyze the ground vibrational state tunneling dynamics. Before the work of Coudert and Hougen, 4.5 .9 no treatment for the concerted quantum tunneling motions existed for the water dimer, and although our present results show significant discrepancies with this model, the generalized IAM treatment is recognized as a valuable step toward obtaining a quantitative understanding of complicated tunneling problems. Briefly, the generalized IAM treatment expresses the VRT energy levels of the water dimer with a phenomenological Hamiltonian of the form

$$
\mathrm{E}(\mathrm{v}, \mathrm{J}, \mathrm{K})=\mathrm{E}_{\mathrm{VIB}}+\mathrm{E}_{\mathrm{E}-\mathrm{O}-\mathrm{E}}+\mathrm{E}_{\mathrm{AT}}+\mathrm{E}_{\mathrm{GDATT}}+\mathrm{E}_{\mathrm{DT}}+\mathrm{E}_{\mathrm{AGDATT}},
$$

for which $E_{V I B}$ is the vibrational contribution, $E_{E-O-E}$ accounts for the end-over-end rotational motion of the dimer frame and the remaining terms describe the tunneling motions within the high barrier approximation such that $E_{\mathrm{AT}}$ is the contribution from acceptor tunneling, EGDAIT and EAGDAIT correspond to geared and antigeared donoracceptor interchange tunneling, respectively, and $E_{D T}$ represents the contribution from donor tunneling. The corresponding energy contributions from each tunneling motion are determined by matrix element expressions which depend on $\mathrm{J}, \mathrm{K}_{\mathrm{a}}$, and the specific tunneling pathway. These expressions define B and higher order distortion constants for each tunneling sublevel as the sum of a structural B with a contribution from each of the isolated tunneling motions. No coupling between inequivalent types of tunneling motions is considered in the high barrier limit, which restricts each coordinate to couple only to its nearest neighbor framework on the IPS, resulting in a set of separable tunneling motions. This treatment provided a unique set of tunneling parameters for each of the four tunneling coordinates $\mathrm{cf}$ the water dimer when the IAM glot $2 ! \mathrm{fit}^{9}$ of the ground state data was 
conducted, and this method has been used to describe the tunneling dynamics in $v=0$ of $\left(\mathrm{H}_{2} \mathrm{O}\right)_{2}$. Similar methods have been utilized in parameterizing tunneling splittings of chemically bound systems such as hydrazine and methylamine. Notation originating from the IAM model has previously been used to define the matrix elements that contribute to each tunneling splitting, yet this notation is avoided within the present discussion because of discrepancies in the model within the context of the observed intermolecular vibrational motion.

In Figure 3.8 the VRT energy levels are summarized pictorially to illustrate the tunneling splittings and symmetry assignments for $\mathrm{K}_{\mathrm{a}}{ }^{\prime \prime}=0,1$, and 2 in $\mathrm{v}=0$ and for $\mathrm{K}_{\mathrm{a}}{ }^{\prime}=$ 0 and 1 in the $82.6 \mathrm{~cm}^{-1}$ vibrationally excited state. The $\mathrm{J}=0 \rightarrow 1$ transitions for each of the 12 bands are represented as arrows to help clarify the conclusions of the analysis section regarding the acceptor and donor acceptor interchange tunneling splittings. The most obvious difference between the ground and vibrational state rotational tunneling manifolds is the large variation of all tunneling splittings with respect to $\mathrm{K}_{\mathrm{a}}$ in the excited state, and this will be discussed below.

\subsubsection{Acceptor Tunneling}

The acceptor tunneling splitting for $v=0$ is specified as $\beta$, for its actual value cannot be determined directly because transitions between $E$ states of different " 1 " and "2" triplets are unlikely, and these have not yet been experimentally observed for the water dimer. A value of $\beta=36 \mathrm{GHz}$ from the $\mathrm{K}_{\mathrm{a}}{ }^{\prime}=0->1$ microwave data of Karyakin et al. 8 has been estimated for this splitting by averaging the contribution of acceptor tunneling in both the $\mathrm{K}_{\mathrm{a}} "=0$ and 1 manifolds. Also, a measure of the change in acceptor tunneling from the $\mathrm{K}_{\mathrm{a}}{ }^{\prime \prime}=1->2$ data of Reference 7 indicates that this splitting increases by $21 \mathrm{GHz}$ in $\mathrm{K}_{\mathrm{a}}{ }^{\prime \prime}=2$ relative to $\mathrm{K}_{\mathrm{a}}{ }^{\prime \prime}=1$. Although the single value of $\beta$ is only valid if acceptor tunneling exhibits a small difference between the $\mathrm{K}_{\mathrm{a}}{ }^{\prime \prime}=0$ and $\mathrm{K}_{\mathrm{a}}{ }^{\prime \prime}=1$ manifolds, it is incorporated along with the measured change of the acceptor tunneling splittings in the 
Figure 3.8 Caption

The complete energy level diagram for both the ground and vibrationally excited states are shown. The $\mathrm{K}_{\mathrm{a}}$ manifolds are vertically aligned and each $\mathrm{J}$ quantum number is labeled. The arrows represent the $R(1)$ transitions for each of the 12 VRT subbands observed, originating from $\mathrm{K}_{\mathrm{a}}{ }^{\prime \prime}=0$ terminating in the excited vibrational state $\mathrm{K}_{\mathrm{a}}{ }^{\prime}=0$ and 1 manifolds. The actual VRT symmetries for each energy levels are labeled from the experimental evidence discussed in the text. For $\mathrm{K}_{\mathrm{a}}>0$ the symmetry species of the asymmetry doublets are labeled such that the lower component is on the left and the upper on the right. For example, in $\mathrm{K}_{\mathrm{a}}{ }^{\prime}=1$ the $1_{11}$ rovibrational state correlates to the $\mathrm{A}_{1}{ }^{+}, \mathrm{E}^{+}$, $\mathrm{B}_{1}^{+}, \mathrm{A}_{2}^{-}, \mathrm{E}^{-}$, and $\mathrm{B}_{2}^{-}$symmetries and the $1_{10}$ correlates to the $\mathrm{B}_{1^{-}}, \mathrm{E}^{-}, \mathrm{A}_{1}^{-}, \mathrm{B}_{2}+, \mathrm{E}^{+}$, and $\mathrm{A}_{2}{ }^{+}$symmetry species. These asymmetry doublets are omitted from the diagram because they are associated with a relatively small splitting. Most of the determinable tunneling splittings for $\mathrm{v}=0, \mathrm{~K}_{\mathrm{a}}=0,1$, and 2 and for the $\mathrm{K}_{\mathrm{a}}=0$ and 1 manifolds of the $82.6 \mathrm{~cm}^{-1}$ vibration are shown. The acceptor tunneling splittings are not drawn to scale and the value of $\beta$ as defined in the text is $36 \mathrm{GHz}$. The donor acceptor interchange splittings are shown and represent the energy separation between the $(+)$ and $(-)$ VRT states for each of the interchange triplets. Donor tunneling shifts are excluded from the figure, due to their small size. 
Figure 3.8

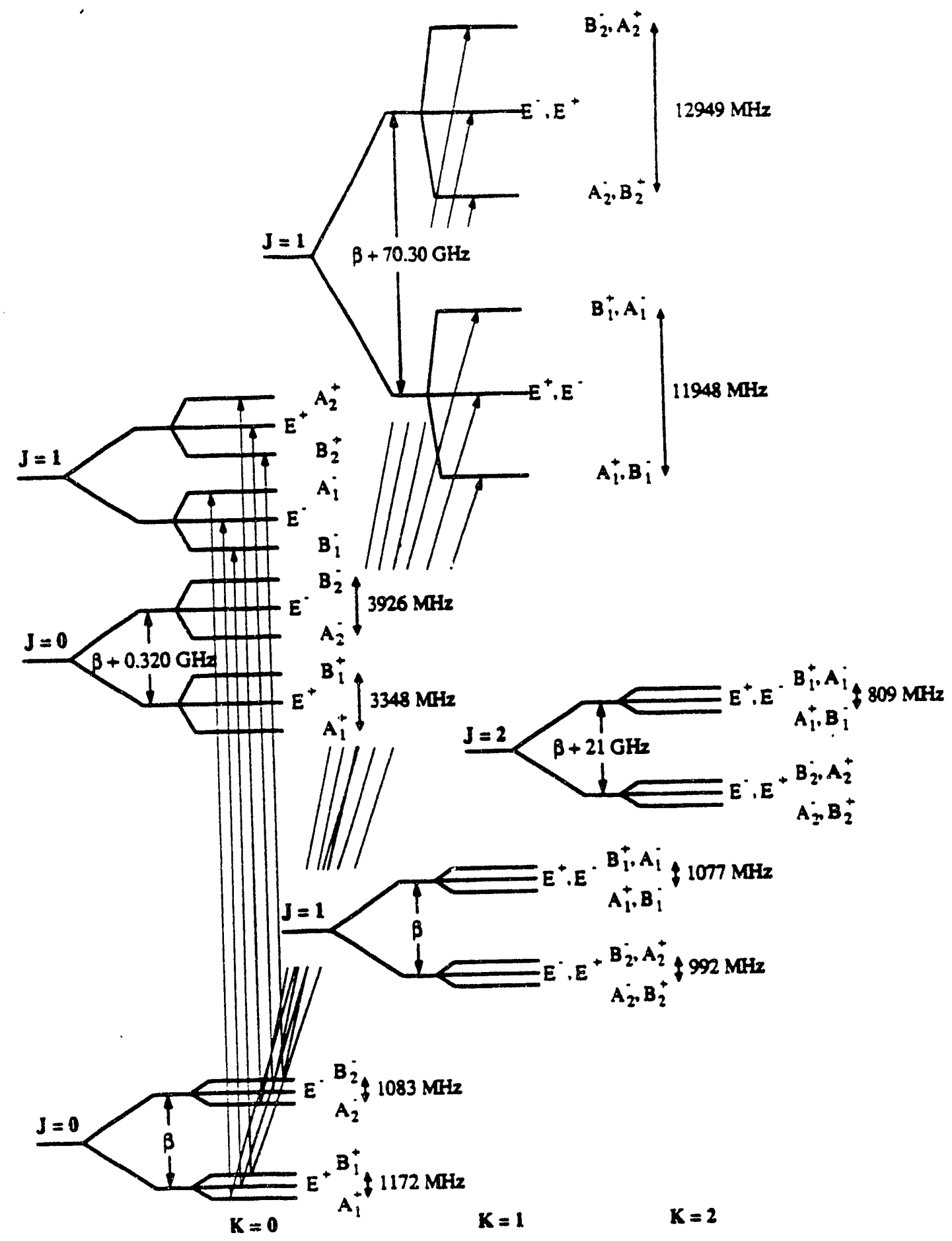


excited state vibration. Acceptor tunneling parameters of $36.3 \mathrm{GHz}$ and $106.3 \mathrm{GHz}$ can then be defined for the two $\mathrm{K}_{\mathrm{a}}$ ' manifolds, since the acceptor tunneling splitting changes by 0.32008 (95) and $70.3000(15) \mathrm{GHz}$ in $\mathrm{K}_{\mathrm{a}}{ }^{\prime}=0$ and 1 , respectively, relative to $\mathrm{K}_{\mathrm{a}}{ }^{\prime \prime}=0$. In Figure 3.4 the two band origins for the $\mathrm{K}_{\mathrm{a}}=0->0$ bands are located in the $2477 \mathrm{GHz}$ spectral region, as discussed earlier, whereas the two sets of triplets corresponding to the $Q$ branches for the $K_{a}=0->1$ bands are evident at frequencies of 2559779.71 (89) and 2630079.7 (20) $\mathrm{MHz}$, designated by the markers (b) and (c) respectively. This spectrum verifies that there is a negligible change in acceptor tunneling for $\mathrm{K}_{\mathrm{a}}{ }^{\prime}=0$ with excitation of the intermolecular vibration, whereas a $70 \mathrm{GHz}$ increase is present in $\mathrm{K}_{\mathrm{a}}{ }^{\prime}=1$ relative to $\mathrm{K}_{\mathrm{a}}{ }^{\prime}=0$.

The ordering of the VRT symmetries about the acceptor tunneling splitting presented in Figure 3.8 for $\left(\mathrm{D}_{2} \mathrm{O}\right)_{2}$ in $\mathrm{v}=0$ is identical to that reported for $\left(\mathrm{H}_{2} \mathrm{O}\right)_{2}$, and it is interesting to compare this ordering with that in the excited vibrational state. In $\mathrm{K}_{\mathrm{a}} "=0$ the symmetry species labeled by "2" defines the upper half of the acceptor tunneling splitting, whereas in $\mathrm{K}_{\mathrm{a}} "=1$ these VRT symmetries characterize the lower half of the acceptor tunneling splitting. The $\mathrm{K}_{\mathrm{a}}{ }^{\prime \prime}=2$ data are also shown in Figure 3.6, and the ordering about the acceptor tunneling splitting for $\left(\mathrm{D}_{2} \mathrm{O}\right)_{2}$, which was originally proposed by $\mathrm{Zwart}$ et al. ${ }^{7}$, has since been corroborated by the recent work of Karyakin et al. ${ }^{8}$ The methylamine-like tunneling coordinate is consistent with the ordering of the symmetry species " 1 " and " 2 " in $\mathrm{K}_{\mathrm{a}}{ }^{\prime}=0$ and 1 , for this tunneling motion generates a spatially oriented structure which requires a rotation about the $A$ inertial axis to reach the symmetrically equivalent form of the dimer. An alternation of " 1 " and " 2 " with $\mathrm{K}_{\mathrm{a}}$ " would thus be expected for this pathway. There is some confusion regarding the predictive capabilities of the IAM model for $\mathrm{K}_{\mathrm{a}}$ " = 2 , yet experimental evidence indicates that the ordering of the energy levels are as shown in Figure $3.8,{ }^{7.8}$ thus the equivalent rotation cannot be used to interpret this $\mathrm{K}_{\mathrm{a}}$ " manifold. The present data verify the ordering about the acceptor tunneling splitting to be the same as for $\mathrm{K}_{\mathrm{a}}{ }^{\prime \prime}=0$ for both the $\mathrm{K}_{\mathrm{a}}{ }^{\prime}=0$ and 1 manifolds, and is in contrast to the $\mathrm{K}_{\mathrm{a}}$ dependence ir 
$v=0$. If the tunneling pathway were to change in the excited vibrational state to a simple rotation of the acceptor subunit about its $C_{2}$ axis, no equivalent rotation of the dimer would be necessary to yield a symmetrically equivalent structure. For this situation the ordering of " 1 " and " 2 " in the various $\mathrm{K}_{\mathrm{a}}$ ' manifolds would be the same, i.e. $\mathrm{K}_{\mathrm{a}}$ ' independent. The two different tunneling pathways, with the equivalent rotations are shown in Figure 3.9. It can thus be concluded that because the $\mathrm{K}_{\mathrm{a}}$-type dependence is very different in the excited vibrational state, a significant change in the acceptor tunneling pathway upon vibrational excitation must be considered. If the $C_{2}$ - type acceptor rotation is responsible for the acceptor tunneling splitting in the excited vibrational state, the breakdown of the high barrier limit would most definitely be realized.

\subsubsection{Donor-Acceptor Interchange Tunneling}

All determinable donor-acceptor interchange splittings are indicated in Figure 3.8 and the differences with respect to $\mathrm{v}$ and $\mathrm{K}$ can be easily recognized. In summary, the donor acceptor interchange splittings are nominally $1 \mathrm{GHz}$ in $\mathrm{v}=0$, and exhibit only a weak dependence with respect to $\mathrm{K}_{\mathrm{a}} "$. Yet in the $82.6 \mathrm{~cm}^{-1}$ excited vibrational state, these splittings increase to $3348.6(16)$ and 3926.1 (18) $\mathrm{MHz}$ in $\mathrm{K}_{\mathrm{a}}{ }^{\prime}=0$ and 11947.7 (18) and 12948.9 (39) in $\mathrm{K}_{\mathrm{a}}$ ' $=1$ for the " 1 " and " 2 " interchange splittings, respectively. This

strong variation with $K_{a}^{\prime}$ can be verified by the labels $(d-f)$ in Figure 3.4, which point to the observed spectral splittings.

The generalized IAM treatrnent of the water dimer predicts that each of the separate interchange splittings for a given $l v, K_{a}>$ is a result of two proposed tunneling motions, viz. geared and antigeared donor-acceptor interchange pathways. The sum of the two matrix elements describing these motions corresponds to the larger splitting labeled with symmetries " 1 ", whereas the difference is found to represent the " 2 " interchange splitting. These coordinates presumably pass through trans and cis transition structures, respectivaly, and these pathways would indicate that the antigeared motion is far less feasible, so its 
Figure 3.9
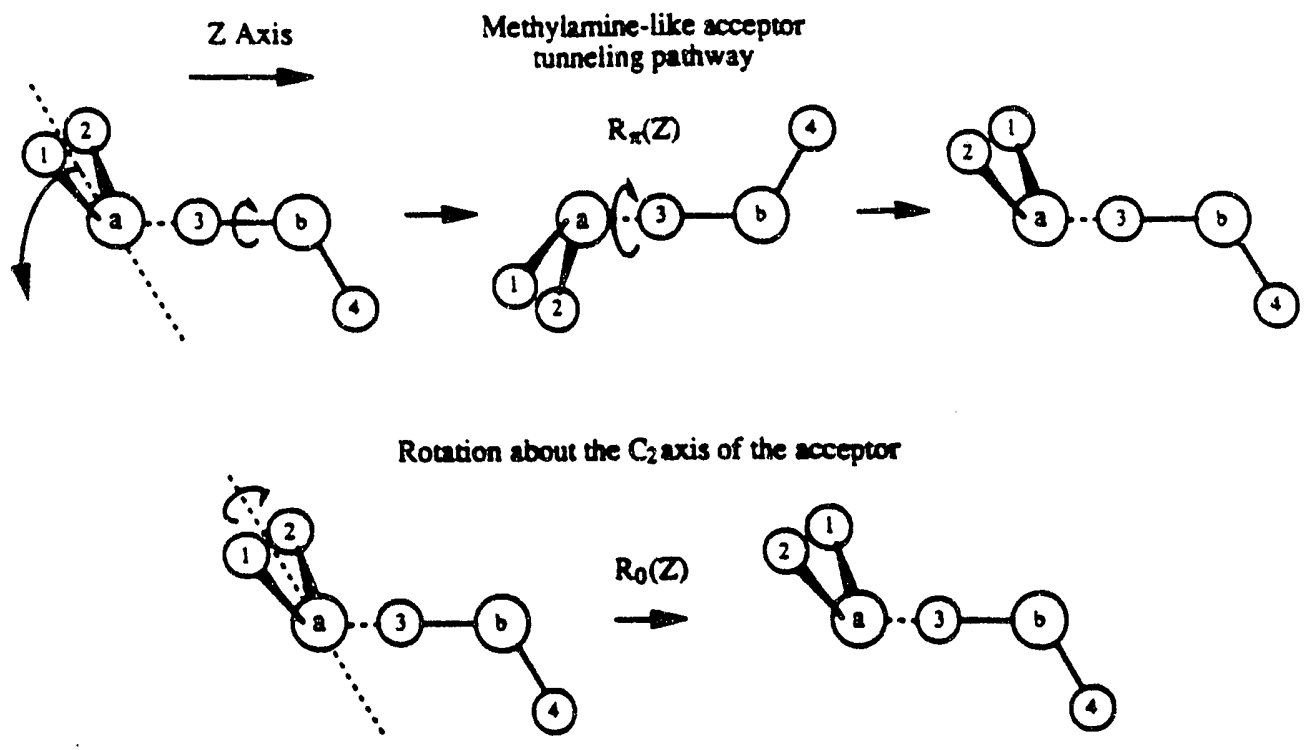

The two types of acceptor tunneling pathways considered along with their corresponding equivalent rotations. The methylamine-like pathway which most likely describes the ground state tunneling path would alternate the ordering of the " 1 " and " 2 " symmetry species about the acceptor tunneling splitting with $K_{a}$, whereas the rotation about the $C_{2}$ axis of the acceptor subunit pathway describes the coordinate for the vibrationally excited state. This is postulated because of the ordering of the "l" and " 2 " symmetry species is $\mathrm{K}_{\mathrm{a}}$ independent.

contribution to the total interchange spliting should be much smaller than that of the geared pathway. Although the difference of the two sets of interchange splittings is in fact small for all $\mathrm{K}_{\mathrm{a}}$ " manifolds, it is easily measurable and the IAM model has adequately explained these results in terms of the two separable tunneling motions. However, it is found that the present vibrational motion reverses the effect that the two proposed interchange matrix elements have on the splitrings relative to the $v=0$ interpretation. Thus, the antigeared matrix element would necessarily have to change sign in the excited state. In other words 
the sum and difference, corresponding to the " 2 " and " 1 " labels of the two interchange splittings, are associated with the opposite VRT symmetry species in both the $\mathrm{K}_{\mathrm{a}}{ }^{\prime}=0$ and 1 manifolds, relative to the ground state.

\subsubsection{Donor Tunneling}

Characterizing a clear trend relating to the effects of donor tunneling on the observed spectra is difficult, but clearly the fitted tunneling parameters do vary considerably with respect to the ground state. These shifts are not shown in Figure 3.8 because of the small magnitude associated with this slow quantum tunneling motion. In $\mathrm{K}_{\mathrm{a}}$ ' $=0$ the value of $\mathrm{EDT}_{\mathrm{DT}}$ is fit as +154 and $+41 \mathrm{MHz}$ for the " 1 " and " 2 " interchange triplets, respectively, whereas for $\mathrm{K}_{\mathrm{a}}{ }^{\prime}=1$ this shift corresponds to fitted values of -333 and $-57 \mathrm{MHz}$. The magnitude for this parameter has consistently been found to be ca. 12-14 $\mathrm{MHz}$ across the three $\mathrm{K}_{\mathrm{a}}$ manifolds sampled in the ground state. Although the absolute value of this shift is as much as an order of magnitude larger in the vibrationally excited state relative to the ground state, the direction of the shift does follow the predictions of the IAM treatment. More precisely, the IAM method predicts that even and odd values of $\mathrm{K}_{\mathrm{a}}$ should yield positive and negative deviations, respectively, as is measured and this can be illustrated by considering the equivalent rotations which correspond to the tunneling path defined in Figure 3.10. Because this tunneling shift is associated with the largest tunneling barrier, and the qualitatively correct $\mathrm{K}_{\mathrm{a}}$ behavior is observed, this motion may still be thought of in terms of the high barrier limit. The large increase in the tunneling shift is probably a result of the intermolecular vibration accessing an energetically higher region of the barrier, although the substantial difference in magnitude found between symmetry species " 1 " and " 2 " cannot be easily rationalized. With the extensive set of excited state data, it immediately becomes clear that the slowly varying tunneling splittings of the ground state become almost chaotic in the intermolecular vibrational state at $82.5 \mathrm{~cm}^{-1}$. 
Figure 3.10

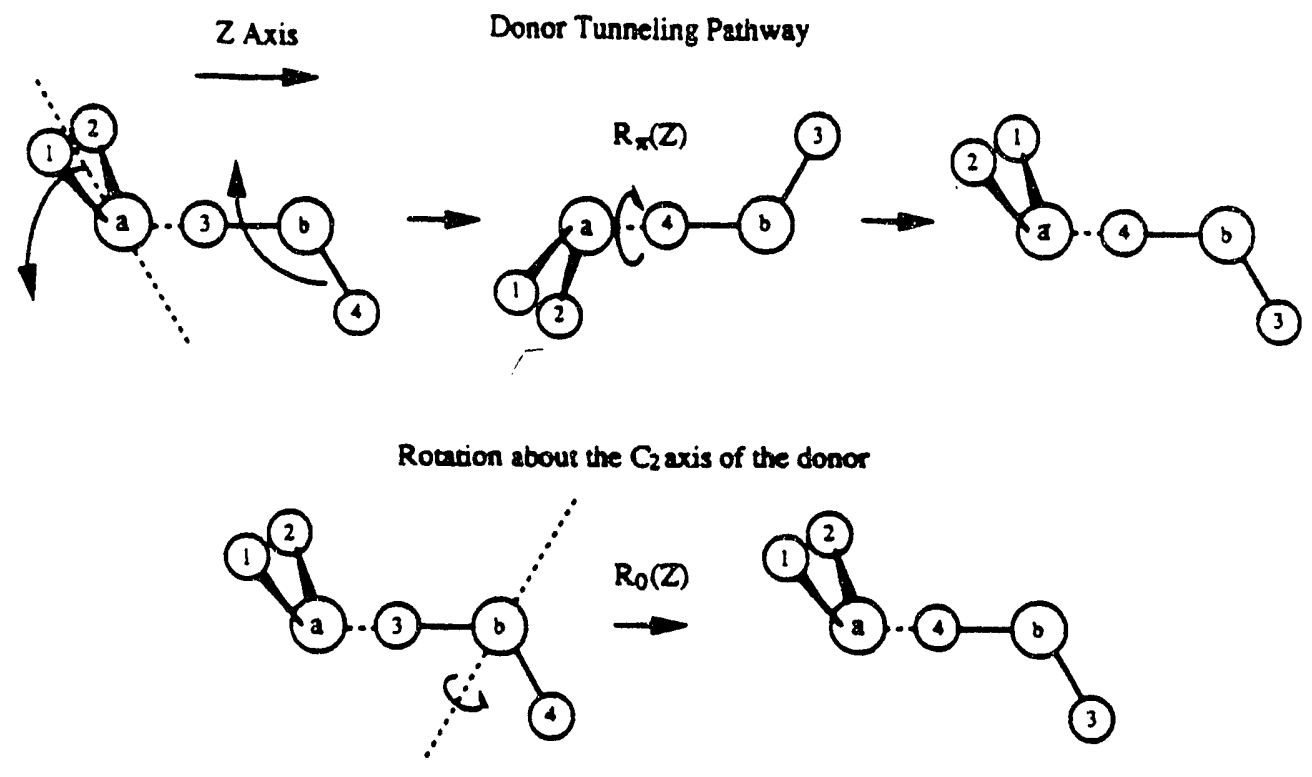

The corresponding equivalent rotations for two donor tunneling pathways are shown. The first pathway is consistent with the $\mathrm{K}_{\mathrm{a}}$ dependence of the donor tunneling shift in both the ground and excited vibrational states. The second pathway, which is not consistent with the data, corresponds with the $R_{0}(Z)$ rotation, would not change the sign of the donor tunneling shift with alternating $K_{a}$. Furthermore, such a motion would presumably be less feasible because it would require the breaking of the $\mathrm{H}$-bond.

\subsection{5 $K_{a}$ Dependence of Tunneling}

In the preceding discussion, the large $\mathrm{K}_{\mathrm{a}}$ ' variation of all tunneling splittings observed in the FIR-VRT spectra has been described. $K_{a}$ dependences in VRT dynamics have been treated previously for systems exhibiting facile quantum tunneling, and two such examples are briefly described here. The first example examines the internal rotationinversion of methylamine, which is analogous to the proposed pathway for acceptor tunneling in the water dimer. In their work, Ohashi et al. ${ }^{20.22}$ fit the observed FIR spectrum to a phenomenological Hamiltonian that includes tunneling parameters which effectively couple the various equivalent frameworks that result from the methyl 
torsion/amine inversion tunneling motion. These parameters are related to matrix elements which depend on the $J$ and $K_{a}$ quantum numbers. These are used to fit the tunneling splittings over a large range of $\mathrm{K}_{\mathrm{a}}$ (up to $\mathrm{K}_{\mathrm{a}}=28$ ), and this method of analysis is similar in principle to an IAM-like treatment. In methylamine, the methyl torsion tunneling splitting exhibits a 20 -fold increase in the first excited vibrational state (at $265 \mathrm{~cm}^{-1}$ ), whereas the amine inversion parameters change only slightly. This observation is used to assign the vibration as a motion along the methyl torsion soordinate. To quantify the effect of the parameterized matrix elements with respect to the complicated $\mathrm{K}_{\mathrm{a}}$ dependence of the tunneling splittings, the strucrural contributions to the energy were subtracted from the total energy of the VRT states by assuming that the tunneling motions sample coordinates that are orthogonal relative to the other degrees of freedom. The remaining energy contributions amounted to the combined internal torsion-inversion energy splitting, which was found to follow a cosine dependence with respect to $\mathrm{K}_{\mathrm{a}}$. The oscillation could be described by a cosine function in which the periodicity depends on a set of fitted tunneling parameters. By fitting these parameters, the experimentally observed period of approximately $4.5 \mathrm{~K}_{\mathrm{a}}$ was reproduced.

All of the tunneling dependencies in the $82.6 \mathrm{~cm}^{-1}$ vibration could, in principle, be characterized with an analogous phenomenological expression if a more complete sampling of the $\mathrm{K}_{\mathrm{a}}$ manifolds were available. In this low temperature molecular beam work, however, such an extensive description of the $\mathrm{K}_{\mathrm{a}}$ dependence will not be possible, so a fit of this kind is not likely to be useful. Also, the treatment of Ohashi et al. ${ }^{20.25}$ is made possible by utilizing the fact that the $265 \mathrm{~cm}^{-1}$ vibrational motion in methylamine directly correlates to the lowest fundamental of the methyl torsion tunneling coordinate. In other words, the vibration samples the torsional coordinate uniquely without the apparent involvement of the inversion tunneling motion. Since all of the water dimer tunneling splittings in the excited vibrational state change dramatically relative to those in $v=0$, such a treatment is not so clearly adaptable to the strongly coupled VRT states of this cluster. 
The geared interchange coordinate of the water dimer may be directly compared with the analogous type of motion in the less complicated hydrogen halide dimers. A large body of theoretical work has been directed at describing the $\mathrm{HCl}$ dimer and the $\mathrm{HF}$ dimer VRT energy levels. The extensive work of Bunker et al. ${ }^{23}$ and the review by Truhlar ${ }^{24}$ represent only a small fraction of the literature relevant to these clusters, yet from this work an appreciation for $\mathrm{K}_{\mathrm{a}}$ - type dependence of the interchange coordinate can be obtained for both dimers. The calculations of Bunker et al. ${ }^{26}$ begin with the construction of an analytical ab initio IPS. A fit of each surface to a functional form provides a potential upon which the VRT levels can be calculated using the semirigid bender formalism. ${ }^{25}$ In the HF dimer, large variations in the donor-acceptor interchange tunneling coordinate with $\mathrm{K}_{\mathrm{a}}$ are found, while for the $\mathrm{HCl}$ dimer the splitting is approximately constant. The PES are very different for the two cases, and Bunker et al. discuss how this may result in the different $\mathrm{K}_{\mathrm{a}}$ dependences in this coordinate. Briefly, in the $\mathrm{HCl}$ dimer the A rotational constant is essentially invariant along this tunneling coordinate, for the calculations show that the dimer never passes through a linear geometry along the minimum energy pathway of this motion. But in the HF dimer, the minimum pathway does pass through the linear configuration, hence the A rotational constant varies considerably and this greatly influences the tunneling splitting within the various $\mathrm{K}_{\mathrm{a}}$ manifolds. From this work it is clear that the angular degrees of freedom accessed by these large amplitude motions is very sensitive to the IPS on which the dynamics occur, and the data presented for the water dimer illustrate a more complicated example of this type of phenomenon.

\subsection{The Intermolecular Vibrational Coordinate}

\subsubsection{The Vibrational . issignment}

Unlike the intermolecular vibrational motions that occur in the hydrogen halide dimers and the methyl torsional motion of methylamine, no particular tunne'ing splitting is uniquely influenced by excitation of the intermolecular vibration observed here for $\left(\mathrm{D}_{2} \mathrm{O}\right)_{2}$. 
In this case, the situation is not consistent with a one dimensional description, wherein the vibrational excitation might correlate directly with a rotation of the water monomers along a particular tunneling coordinate. A phenomenological approach, such as the method of Ohashi et al. ${ }^{20.25}$ for methylamine or the generalized IAM treatment ${ }^{9}$, could possibly parameterize the tunneling splittings by considering periodically värying $\mathrm{K}_{\mathrm{a}}$ dependencies for each tunneling motion, yet it is unlikely that such a treatment would provide much insight into the nature of the vibrational coordinate. Because of the inseparability of the multidimensional tunneling motions from that of the intermolecular vibraxion, an unambiguous characterization of the vibrational mode is not possible within the context of the present FIR-VRT analysis. The symmetry of the $82.6 \mathrm{~cm}^{-1}$ vibration was previously assigned as $\mathrm{B}_{1}{ }^{+}$from the spectral eviuence available at that time. ${ }^{10}$ This assignment is not supported by the additional observations reported here. The previous analysis indicated that the acceptor bend was an appropriate vibrational assignment, for this motion correlates to the $\mathrm{B}_{1}{ }^{+}$symmetry species in $\mathrm{G}_{16}$. However, the present data show that an appreciable change in the acceptor tunneling splitting occurs only with the excitation of angular momentum about the $\mathrm{A}^{\prime}$ inertial axis. Therefore, it is possible that the pure vibrational motion does not correlate to the methylamine-like acceptor tunneling pathway.

Figure 3.8 is an energy level diagram corresponding to an apparent vibrational symmetry belonging to the $A_{1}+$ irreducible representation in $G_{16}$. This is assigned as such because the ordering of the VRT symmetries in $\mathrm{K}_{\mathrm{a}}{ }^{\prime}=0$ is determined to be identical to those of $\mathrm{K}_{\mathrm{a}}{ }^{\prime}=0$. The fundamental of a totally symmetric vibrational motion, such as the intermolecular stretch, would correlate to this symmetry species. However, a fairly reliable harmonic frequency estimate for this motion of $145 \mathrm{~cm}^{-1}$ has been determined from the analysis of ground state distortion constants, ${ }^{2}$ hence it seems unlikely that this would be an appropriate assignment. Another assignment, also consistent with the lowest symmetry species of $\mathrm{A}_{1}^{+}$, might correspond to an overtone in one of the tunneling coordinates possessing an even number of vibrational quanta. However, any tunneling motion would 
be a candidate for such an assignment, and again, no single motion can be uniquely related to this strongly coupled VRT band.

For the purpose of this discussion, let it be assumed that the high barrier limit is appropriate, so that each tunneling motion is in fact separable from all other coordinates for the excited vibrational state In this limit the transition can be considered to correspond to an excitation of the vibrational mode which correlates to a particular tunneling coordinate. From this perspective, the observed vibration could be rationalized as a motion similar to donor-acceptor interchange, for its associated splitting exhibits a 3-4 fold increase in $\mathrm{K}_{\mathrm{a}}{ }^{\prime}=$ 0 relative to that in the vibrational ground state, whereas the acceptor tunneling splitting indicates little variation (in $\mathrm{K}_{\mathrm{a}}{ }^{\prime}=0$ ) with respect to vibrational excitation. Although such an assignment can be justified in this limit by the measured tunneling splittings, the apparent vibrational symmetry of $\mathrm{A}_{1}{ }^{+}$( $\mathrm{a}^{\prime}$ in the $\mathrm{C}_{\mathrm{S}}(\mathrm{M})$ limit) is not appropriate for an out of plane motion (correlating to an $a^{\prime \prime}$ mode), and the interchange pathway is predicted 22 to take the dimer through a transition structure of $C_{i}$ symmety via a trans-type bending coordinate.

Without information pertaining to the larger values of $\mathrm{K}_{\mathrm{a}}$, it seems unlikely that an IAM-like treatment would be useful in rationalizing the effects of the intermolecular vibration, for the data show that the associated splitting in $K_{a}=1$ is comparable to the $A$ rotational constant. In particular, the acceptor and the antigeared donor-acceptor interchange tunneling motions are most affected by this breakdown. It also seems possible that the acceptor tunneling motion follows a different pathway in the excited vibrational state, which would further enhance the picture in which the cluster dynamics involve a high degree of angular coupling between an highly anharmonic vibration and potentially all of the tunneling coordinates. Under these circumstances, the high barrier limit is inapplicable, and the tunneling motions are not separable from the rovibrational Hamiltonian, as is the case for van der Waals molecules which are best described in the free rotor limit such as $\mathrm{Ar}-\mathrm{H}_{2} \mathrm{O}^{26}, \mathrm{Ar}-\mathrm{NH}_{3}{ }^{27}, \mathrm{Ar}-\mathrm{HCl}^{28}$ and $\mathrm{Ar}_{2}-\mathrm{HCl}^{29}$. 
Until now, matrix isolation experiments ${ }^{30}$, guided by theoretically based predictions have been the only means for characterizing the intermolecular degrees of freedom. Many pair potentials exist for the water dimer, ${ }^{31}$ but it is difficult to determine which which of these are appropriate for predicting band origins of the intermolecular vibrations. When examining the available ab initio surfaces as to their probable reliability in light of the present data, it is noted that the calculated frequencies vary from ca. $80-160$ $\mathrm{cm}^{-1}$ for the lowest two intermolecular vibrational modes, and it becomes clear that no particular IPS stands out as the most suitable surface. Although the vibrational frequencies vary substantially, the lowest modes are generally predicted to be either an acceptor bend $\left(\mathrm{a}^{\prime}\right)$ or a donor torsion (a"). These predirtions are consistent with the $82.6 \mathrm{~cm}^{-1}$ vibration characterized here and the probable existence of a vibration at slightly higher frequency, (ca. $>88 \mathrm{~cm}^{-1}$ ) as is evidenced by the Coriolis perturbed $2630 \mathrm{GHz}, \mathrm{K}_{\mathrm{a}}$ ' $=1$ states of " 2 " symmetry. As discussed in Section III, this perturbation would necessarily be from a $\mathrm{K}_{\mathrm{a}}=$ 0 manifold having the " 2 " symmetry labels, because of the selection rules governing the Coriolis interaction outlined in Section III. If these states were associated with the lower half of the acceptor tunneling splitting of the perturbing vibration, then that motion would transform as an a" mode in $\mathrm{C}_{\mathrm{s}}(\mathrm{M})$, whereas the present vibration appears to be of $\mathrm{a}^{\prime}$ symmetry $\left(\mathrm{A}_{1}{ }^{+}\right.$transforms as $\mathrm{a}^{\prime}$ in in $\left.\mathrm{C}_{5}(\mathrm{M})\right)$ and this would be consistent with assigning the vibration as the acceptor bend.

\subsubsection{Isotopically Substituted VRT Bands for the Water Dimer}

By observing spectra for different isotopomers of the water dimer a clear picture of the individual tunneling splittings may result, and with a characterization of the band origin for each, conclusions may be proposed for the vibrational coordinate. This section will be illustrate the latter of these two poincs. When realizing that isotopic substitution may occur on either the donor or the acceptor monomers, it becomes clear that a great deal of information exists for each vibrational state when including these species. The lowest 
frequency VRT transitions for $v=0$ have been reported for all of the isotopomers, 32 and these data are reproduced in Table 3.4 .

There are two important reasons for examining isotopically substituted water dimer species. First of all, the tunneling splittings are different for each of the various isotopomers because the tunneling pathways presented in Section 3.3 do not exchange identical particles. For example, the geared donor-acceptor interconversion tunneling for the isotopomer $\mathrm{D}_{2} \mathrm{O}$ - DOH produces a structure of the form DHO - DOD which is clearly not identical to the original. The group theory for each of these clusters has been discussed in Reference 32 and only the relevant symmetry considerations for a particular molecule will

Table 3.4

Microwave frequencies for deuterated water dimer species taken from Reference 32.

\begin{tabular}{ccclc}
\hline \hline Species & $\begin{array}{c}\text { \# of } \\
\text { Frameworks }\end{array}$ & $\mathrm{J}^{\prime}-\mathrm{J}^{\prime \prime}$ & \multicolumn{1}{c}{ Frequencies } \\
\hline \multirow{2}{*}{$\mathrm{H}_{2} \mathrm{O}-\mathrm{DOH}$} & 2 & $1-0$ & 12239.001 & 12312.521 \\
& $2-1$ & 24479.057 & 24623.903 \\
$\mathrm{D}_{2} \mathrm{O}-\mathrm{DOH}$ & 2 & $1-0$ & 11345.252 & 11349.749 \\
& 2 & $2-1$ & 22689.670 & 22698.457 \\
$\mathrm{H}_{2} \mathrm{O}-\mathrm{DOD}$ & 4 & $1-0,1-0$ & $11785.312, \ldots$ & $11789.484,11789.501$ \\
& & $2-1,2-1$ & $23569.655,23569.782$ & $23577.787,23577.985$ \\
$\mathrm{HDO}-\mathrm{DOD}$ & 4 & $1-0$ & 11296.385 & $\ldots$ \\
\hline
\end{tabular}

be considered below. Secondly, the frequency shift of the band origin for a particular vibration, due to the replacement of deuteriums with hydrogens will be indicative of the type of vibrational motion.

In hopes of finding spectra for the isotopomers of the water dimer possessing a mixture of deuterium and hydrogen atoms, a pipet full $(5 \mathrm{ml})$ of $\mathrm{H}_{2} \mathrm{O}$ was occasionally added to the $\mathrm{D}_{2} \mathrm{O}$ sample. Unfortunately this mixture was not used consistently, and resulted in only partial VRT spectra for one of the isotopomers. Both a $\mathrm{K}_{\mathrm{a}}=0 \rightarrow 0$, and $\mathrm{K}_{\mathrm{a}}=0 \rightarrow 1$ band were observed and are reported in Table 3.5 along with the fitted 
Table 3.5

The two observed VRT bands for the $\mathrm{D}_{2} \mathrm{O}$ - DOH isotopomer of the water dimer and the fixied rotational constants. All reported transitions and constants are in $\mathrm{MHz}$.

\begin{tabular}{cccc}
\multicolumn{4}{c}{$\mathrm{K}_{\mathrm{a}}=0$ - $>0$ Band } \\
\cline { 2 - 4 } & $\mathrm{J}^{\prime \prime}$ & Frequency & $0-\mathrm{C}$ \\
\hline \hline 4 & 5 & 2423694.3 & -1.3 \\
3 & 4 & 2435231.0 & -1.2 \\
1 & 2 & 2458158.0 & -1.0 \\
1 & 0 & 2492204.6 & -1.1 \\
2 & 1 & 2503461.9 & 1.5 \\
3 & 2 & 2514664.8 & 1.7 \\
4 & 3 & 2525811.5 & 2.3 \\
5 & 4 & 2536894.0 & 1.2 \\
6 & 5 & 2547903.3 & -3.3 \\
7 & 6 & 2558843.5 & 1.2 \\
8 & 7 & 2569690.3 & 0.1
\end{tabular}

\begin{tabular}{cccc}
\multicolumn{4}{c}{$\mathrm{K}_{\mathrm{a}}=0->1$ Band } \\
\hline \multicolumn{1}{l}{$\mathrm{J}^{\prime \prime}$} & Frequency & $0-\mathrm{C}$ \\
\hline \hline 5 & 6 & 2605842.6 & -2.2 \\
4 & 5 & 2617108.4 & -0.1 \\
3 & 4 & 2628391.8 & 0.2 \\
1 & 1 & 2673641.2 & -2.8 \\
2 & 2 & 2673539.8 & -2.4 \\
3 & 3 & 2673387.7 & -2.0 \\
4 & 4 & 2673185.2 & -1.4 \\
5 & 5 & 2672932.0 & -1.1 \\
6 & 6 & 2672628.7 & -1.0 \\
7 & 7 & 267276.2 & -0.3 \\
8 & 8 & 2671874.8 & 0.7 \\
9 & 9 & 2671424.1 & 1.2 \\
1 & 0 & 2685058.8 & 0.7 \\
2 & 1 & 2696435.1 & 0.9 \\
3 & 2 & 2707825.7 & 3.2 \\
4 & 3 & 2719226.3 & 4.2 \\
5 & 4 & 2730637.2 & 5.0 \\
6 & 5 & 2742055.2 & 3.0 \\
7 & 6 & 2753475.3 & -6.1
\end{tabular}

Fit of bands Yields

$B^{\prime \prime}=5674.701(20)$

$\mathrm{D}^{\prime \prime}=0.0410(12)$

$\mathrm{K}_{\mathrm{i}}^{\prime} \equiv 0$ State

$v_{0}=2480902.7(19)$

$B^{\prime}=5674.701(20)$

$\mathrm{D}^{\prime}=0.0928(21)$
$K_{i}^{\prime} \equiv 1$ State

$$
\begin{aligned}
& v_{0}=2679360.31(98) \\
& B^{\prime}=5665.367(65) \\
& D^{\prime}=0.0386(14) \\
& (B-C)^{\prime}=64.837(77)
\end{aligned}
$$

Standard Deviation of Fit $=2.2 \mathrm{MHz}$

rotational constants. It is readily noticed that the ground state B rotational constant of 5674.7 most reasonably matches the data for the $\mathrm{D}_{2} \mathrm{O}$ - DOH complex shown in the right hand column of Table 3.4. In this molecule each rovibrational state splits into an $A_{1}$ and an 
$A_{2}$ state due to acceptor tunneling, and these symmetry species alternate from + to - and from - to + for even and odd J, respectively. Finally, the nuclear spin weights for the symmetry species $A_{1}+/ / A_{2}-/+$ are 2 and 1 , respectively, and the selection rules are $\Gamma_{i}^{+}<->$ $\Gamma_{\mathrm{i}} \cdot$

From the data presented in Table 3.5, the actual symmetiy of the states cannot be rigorously determined, and the since only half of the VRT data exists no information regarding the change in acceptor tunneling is currently available. A calculated value for the structural A rotational constant of $130 \mathrm{GHz}$ indicates that a large change in the acceptor tunneling splitting with the $\mathrm{K}_{\mathrm{a}}$ ' quantum number may be present, since the frequency separation of the $\mathrm{K}_{\mathrm{a}}=0 \rightarrow 0$ and $\mathrm{K}_{\mathrm{a}}=0 \rightarrow 1$ VRT subbands is approximately $200 \mathrm{GHz}$. However, this must be verified by identifying the other two subbands for the cluster. Although these data are incomplete, they further support the vibrational assignment corresponding to that of the acceptor bend, because the fitted band origin for the $K_{2}=0 \rightarrow$ 0 band of $2480902.7 \mathrm{MHz}$ is nearly identical to those reported for $\left(\mathrm{D}_{2} \mathrm{O}\right)_{2}$ indicating that isotopic substitution of the donor subunit does not substantially shift the vibrational band origin relative to the perdeuterated species. Thus, the mass of the donor does not strongly influence the vibrational coordinate.

\subsubsection{Summary of VRT Analysis}

In summary, the tunneling splittings for the $82.6 \mathrm{~cm}^{-1}$ vibrational motion in the $\mathrm{d}_{4}$ isotopomer of the water dimer have been characterized in detail, and are found to exhibit substantial variations relative to their analogues in $v=0$, possibly evidencing large changes in the actual tunneling pathways for the lowest barrier motions. Strong $\mathrm{K}_{\mathrm{a}}$ type dependences exist for all motions, as has been demonstrated for other similar highly anharmonic tunneling systems. However, in the present case, the large amplitude tunneling motions can not be easily separated from the vibrational motion, thus the relative contributions of the various terms in the water dimer VRT Hamiltonian are no longer clear 
and the dynamics must be treated in a more extensive manner. By considering the a and $c$ type transitions, the symmetry of the lowest $\mathrm{K}_{\mathrm{a}}{ }^{\prime}=0$ VRT states, the evidence of a perturbing state to higher frequency, and the $\mathrm{D}_{2} \mathrm{O}$ - DOH VRT data, the vibration has been assigned to the acceptor bending coordinate of a' symmetry. This vibration has been predicted to be one of the lowest lying excited state vibrations for the water dimer with the current high level ab initio theory, however, it appears that characterizing the effects of highly anharmonic vibrations on the tunneling dynamics will require the development of new descriptive models which take the IPS of the cluster more thoroughly into consideration. The challenge of reproducing such widely varying tunneling effects is a formidable, one and will certainly require high accuracy in an IPS. Such high level ab initio treatments, coupled with a rigorous calculation of the VRT dynamics, could possibly elucidate the dynamics of quantum tunneling in the intermolecular vibrations of the water dimer, help to deduce the forces which contribute to the large changes in tunneling pathways upon vibrational excitation, and will certainly aid in the assignment of the intermolecular vibrational modes accessible with the FIR spectrometer. A six dimensional calculation of this kind has been recently accomplished by van Bladel et al., 33 producing qualitative agreement of the VRT states for the ground vibrational state of the well characterized ammonia dimer, which exhibits tunneling among 72 symmetrically equivalent minima. ${ }^{34}$ For this calculation, the dynamics are explicitly calculated on a known ab initio IPS $\left(\mathrm{V}_{\text {int }}\right)$ with a basis set of Wigner rotation matrices. The potential is expanded in terms of this basis through the following expressions,

$$
\begin{aligned}
& V_{\text {int }}\left(R, \phi_{A}, \theta_{A}, \chi_{A}, \phi_{B}, \theta_{B}, \chi_{B}\right)=\sum_{\Lambda} V_{\Lambda}(R) A_{\Lambda}\left(\phi_{A}, \theta_{A}, \chi_{A}, \phi_{B}, \theta_{B}, \chi_{B}\right) \\
& A_{\Lambda}\left(\phi_{A}, \theta_{A}, \chi_{A}, \phi_{B}, \theta_{B}, \chi_{B}\right)=\sum_{M}\left(\begin{array}{lll}
L_{A} & L_{B} & L \\
M & -M & 0
\end{array}\right) \\
& \times D_{M K_{A}}^{\left(L_{A}\right) *}\left(\phi_{A}, \theta_{A}, \chi_{A}\right) D_{-M_{B}}^{\left(L_{B}\right) *}\left(\phi_{B}, \theta_{B}, \chi_{B}\right)
\end{aligned}
$$


In these expressions $V_{\text {int }}$ is the IPS over all of the radial and angular coordinates of the two polyatomic monomers a and $b, V_{\Lambda}(R)$ are the expansion coefficients for the angular functions $A_{\Lambda}\left(\phi_{A}, \theta_{A}, \chi_{A}, \phi_{B}, \theta_{B}, \chi_{B}\right)$, the expression in brackets is a Wigner $3 j$ symbol, and the D's are Wigner rotation matrices. Although the results of this calculation are currently limited by available computer technology, which prohibits the full convergence of the energy. levels, such in approach could yield the qualitative information necessary to understand the complicated multidimensional tunneling dynamics in hydrogen bonded clusters and their coupling to intermolecular vibrational motions.

\subsubsection{Basis Functions}

The first step for a calculation of this kind for the water dimer, requires the determination of the symmetrized basis functions in the appropriate molecular symmetry group $\mathrm{G}_{16}$. To do this, the method of van Bladel ${ }^{35} \mathrm{et}$ al. is used to determine the affect of each symmetry operation in $\mathrm{G}_{16}$ on the three Euler angles of each monomer. Briefly, a right handed space fixed coordinate system is defined at the center of mass of the dimer. From this axis system, coordinate vectors which describe the position of each atom in the

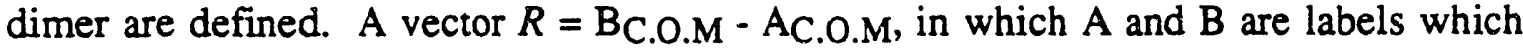
distinguish between the two water monomers, defines the dimer frame, and will account for end-over-end rotation of the complex. The effect of each permutation inversion operation when applied to the polar angles $\alpha$ and $\beta$ of $R$ (dimer fixed frame with respect to the space fixed frame) is then characterized. It is found that the only $E^{*}$ and the interchange of $A$ with $B$ (with an operation containing the (ab) permutation) results in a transformation of $R$ from $R \rightarrow-R$, and this transforms the polar angles from $\alpha>\alpha+\pi$, and $\beta \rightarrow \pi-\beta$. Finally, a coordinate system on each of the monomers is defined from which the Euler angles for each monomer may be referenced. The effect of each permutation inversion operation is then determined for the Euler angles, using the algebraic method outlined in the 
Appendix of Reference 35. This method results in the transformation properties of the angles which characterize end-over-end rotation, and those which define the internal rotation of each monomer $\left(\phi_{A}, \theta_{A}, \chi_{A}\right.$ and $\left.\phi_{B}, \theta_{B}, \chi_{B}\right)$, with respect to each of the permutation inversion operations. These transformation properties are summarized in Table 3.6.

The calculation would consist of finding the VRT eigenstates on the IPS which has been converted to the appropriate rombination of $D$ matrices through Equations 3.7 and 3.8. The primitive basis functions would consider end-ove-end rotation and both internal rotors, and this is also most effectively described with a basis of $\mathrm{D}$ matrices. The primitive basis functions will take the form

$$
\begin{gathered}
C_{n o r m} D_{\Omega_{A} k_{A}}^{\left(j_{A}\right) *}\left(\phi_{A}, \theta_{A}, \chi_{A}\right) D_{\Omega_{B} k_{B}}^{\left(j_{B}\right) *}\left(\phi_{B}, \theta_{B}, \chi_{B}\right)\left\langle j_{A a} \Omega_{A} j_{B} \Omega_{B}|j \Omega| D_{M \Omega}^{(\jmath) *}(\alpha, \beta, 0)\right. \\
=1 j_{A} k_{A} j_{B} k_{B} j \Omega J M>
\end{gathered}
$$

In Equation 3.9 the first two D functions represent those for the internal rotors, $A$ and $B$, the expression in brackets is a Clebsch Gordon coefficient, and the third D function represents end-over-end rotation of the cluster frame. The ket notation will be used to illustrate the operation of any permutation inversion operation of $\mathrm{G}_{16}$ on this basis function, and in Table 3.7 the results of this are presented. Each of the quantum numbers are defined in the table caption. Linear combinations of these functions would then be used to symmetrize the calculation so that the Hamiltonian matrix $<\Phi_{\mathrm{f}}|\mathrm{H}| \Phi_{\mathrm{i}}>$ would become block diagonal for which each block would correspond to a particular symmetry species, defined by the irreducibia representations in $\mathrm{G}_{16}$. This procedure is necessary to minimize the size of the Hamiltonian matrix, because each symmetry block could be diagonalized separately, rather than diagonalizing the entire matrix at once.

The next step is to expand the potential according to Equations 3.7 and 3.8. This was accomplished for a Hartree Fock ab initio potential. 36 Unfortunately, the large 
Table 3.6

The transformation of all angular coordinates of the water dimer under each of the permutation inversion operations of the $\mathrm{G}_{16}$ molecilar symmetry group. The end-over-end rotation of the dimer frame is described by the polar angles $\alpha$ and $\beta$. The two internal water rotors are described by the three Euler angles on each of the monomers a and $b$. All angles are referred to a space fixed coordinate system.

\begin{tabular}{|c|c|c|c|c|c|c|c|c|}
\hline & $\alpha$ & $\beta$ & $\phi_{\mathrm{a}}$ & $\theta_{\mathrm{a}}$ & $x_{a}$ & $\phi_{b}$ & $\theta_{\mathrm{b}}$ & $\chi_{b}$ \\
\hline$E$ & $\alpha$ & $\beta$ & $\phi_{\mathrm{a}}$ & $\theta_{\mathrm{a}}$ & $\chi_{a}$ & $\phi_{b}$ & $\theta_{b}$ & $\chi_{b}$ \\
\hline (12) & $\alpha$ & $\beta$ & $\phi_{\mathrm{a}}$ & $\theta_{\mathrm{a}}$ & $x_{a}-\pi$ & $\phi b$ & $\theta_{\mathrm{b}}$ & $\chi_{b}$ \\
\hline (34) & $\alpha$ & $\beta$ & $\phi_{\mathrm{a}}$ & $\theta_{\mathrm{a}}$ & $\chi_{\mathrm{a}}$ & $\phi_{b}$ & $\theta_{\mathrm{b}}$ & $\chi_{b}-\pi$ \\
\hline$(12)(34)$ & $\alpha$ & $\beta$ & $\phi_{\mathrm{a}}$ & $\theta_{\mathrm{a}}$ & $\chi_{a}-\pi$ & $\phi_{\mathrm{b}}$ & $\theta_{\mathrm{b}}$ & $\chi_{b}-\pi$ \\
\hline$a b(13)(24)$ & $\alpha+\pi$ & $\pi-\beta$ & $-\phi_{0}$ & $\pi-\theta_{b}$ & $\chi_{b}-\pi$ & $-\phi_{a}$ & $\pi-\theta_{a}$ & $\chi_{a}-\pi$ \\
\hline$a b(14)(23)$ & $\alpha+\pi$ & $\pi-\beta$ & $-\phi_{b}$ & $\pi-\theta_{b}$ & $-\chi_{b}$ & $-\phi_{a}$ & $\pi-\theta_{a}$ & $-x_{a}$ \\
\hline$a b(1324)$ & $\alpha+\pi$ & $\pi-\beta$ & $-\phi_{b}$ & $\pi-\theta_{b}$ & $\chi_{b}-\pi$ & $-\phi_{a}$ & $\pi-\theta_{a}$ & $-\chi_{a}$ \\
\hline$a b(1423)$ & $\alpha+\pi$ & $\pi-\beta$ & $-\phi_{b}$ & $\pi-\theta_{b}$ & $-\chi_{b}$ & $-\phi_{a}$ & $\pi-\theta_{\mathrm{a}}$ & $\chi_{a}-\pi$ \\
\hline$E^{*}$ & $\alpha+\pi$ & $\pi-\beta$ & $-\phi_{a}$ & $-\theta_{a}$ & $\pi-\gamma_{a}$ & $-\phi_{b}$ & $-\theta_{b}$ & $\pi-\chi_{b}$ \\
\hline$(12)^{*}$ & $\alpha+\pi$ & $\pi-\beta$ & $-\phi_{a}$ & $-\theta_{\mathrm{a}}$ & $-\chi_{a}$ & $-\phi_{b}$ & $-\theta_{b}$ & $\pi-\chi_{b}$ \\
\hline$(34)^{*}$ & $\alpha+\pi$ & $\pi-\beta$ & $-\phi_{a}$ & $-\theta_{\mathrm{a}}$ & $\pi-\chi_{a}$ & $-\phi_{b}$ & $-\theta_{b}$ & $-\chi_{b}$ \\
\hline$(12)(34)^{*}$ & $\alpha+\pi$ & $\pi-\beta$ & $-\phi_{a}$ & $-\theta_{\mathrm{a}}$ & $-\chi_{a}$ & $-\phi_{b}$ & $-\theta_{\mathrm{b}}$ & $-\chi_{b}$ \\
\hline$a b(13)(24)^{*}$ & $\alpha$ & $\beta$ & $\phi_{\mathrm{b}}$ & $\theta_{b}-\pi$ & $-\chi_{b}$ & $\phi_{2}$ & $\theta_{a}-\pi$ & $-\chi_{a}$ \\
\hline$a b(14)(23) *$ & $\alpha$ & $\beta$ & $\phi_{\mathrm{b}}$ & $\theta_{b}-\pi$ & $\pi-\chi_{b}$ & $\phi_{\mathrm{a}}$ & $\theta_{a}-\pi$ & $\pi-\chi_{a}$ \\
\hline$a b(1324)^{*}$ & $\alpha$ & $\beta$ & $\phi_{\mathrm{b}}$ & $\theta_{b}-\pi$ & $-\chi_{b}$ & $\phi_{a}$ & $\theta_{\mathrm{a}}-\pi$ & $\pi-\chi_{a}$ \\
\hline$a b(1423)^{*}$ & $\alpha$ & $\beta$ & $\phi_{\mathrm{b}}$ & $\theta_{b}-\pi$ & $\pi-\chi_{b}$ & $\phi_{a}$ & $\theta_{a}-\pi$ & $-\chi_{\mathrm{a}}$ \\
\hline
\end{tabular}


Table 3.7

Ine result of each operation in $\mathrm{G}_{16}$ on the primitive basis function of 3.9. These are obtained by substituting the transiormed angles listed in Table 3.6 into Equation 3.9 and using the identities for D matrices found in Zare 37 and Loeser. 38

E

$a b(13)(24)$

$a b(14)(23)$

$\mathrm{ab}(1324)$

$a b(1423)$

$E^{*}$

$(12)(34)^{*}$

$\mathrm{ab}(13)(24) *$

$a b(14)(23)^{*}$

$\mathrm{ab}(1324)^{*}$

$\mathrm{ab}(1423)^{*}$ $\mid \mathrm{ja}_{\mathrm{a}} \mathrm{k}_{\mathrm{a}} \mathrm{jb}_{\mathrm{b}} \mathrm{k}_{\mathrm{b}} \mathrm{j} \Omega \mathrm{J} \mathrm{M}>$ $(-1)^{k_{a}} \mid j_{a} k_{a} j_{b} k_{b} j \Omega J M>$ $(-1)^{k b} \mid j_{a} k_{a} j b k_{b} j \Omega J M>$ $(-1)^{k a+k b} \mid j_{a} k_{a} j_{b} k_{b} j \Omega J M>$ $(-1)^{J+j a+j b} \mid j_{a} k_{a} j b k_{b} j-\Omega J M>$ $(-1)^{j+j a+j b+k a+k b} \mid j_{a} k_{a} j_{b} k_{b} j-\Omega J M>$ $(-1)^{\mathrm{J}+\mathrm{ja}+\mathrm{jb}+\mathrm{kb}} \mid \mathrm{ja}_{\mathrm{a}} \mathrm{k}_{\mathrm{a}} \mathrm{jb}_{\mathrm{b}} \mathrm{k}_{b} \mathrm{j}-\Omega \mathrm{J} \mathrm{M}>$ $(-1)^{J+j a+j b+k a} \mid j_{a} k_{a} j b k_{b} j-\Omega J M>$ $(-1)^{J+j+j a+j b+k a+k b} \mid j a-k_{a} j b-k_{b} j-\Omega J M>$ $(-1)^{J+j+j a+j b+k b} \mid j a-k_{a} j b-k_{b} j-\Omega J M>$ $(-1)^{\mathrm{J}+j+j a+j b+k a} \mid j_{a}-k_{a} j b-k_{b} j-\Omega J M>$ $(-1)^{\mathrm{J}+j+j a+j b} \mid j a-k_{a} j b-k_{b} j-\Omega J M>$ $(-1)^{j+k a+k b} \mid j a-k_{a} j b-k_{b} j \Omega J M>$ $(-1) j \mid j a-k_{a} j b-k_{b} j \Omega J M>$ $(-1)^{j+k_{a}} \mid j_{a}-k_{a} j_{b}-k_{b} j \Omega J M>$ $(-1)^{j+k b} \mid j_{a}-k_{a} j b-k_{b} j \Omega J M>$

anisotropy in the potential prohibited reproduction of the original IPS with the expanded version to within $70 \%$, even when using values of $\mathrm{L}_{A}=\mathrm{L}_{B}=7$. When comparing this accuracy with the $\left(\mathrm{NH}_{3}\right)_{2}$ calculation (reproducible to within $95 \%$ ), and realizing that the ground state VRT levels did not adequately converge by incrementally increasing the basis set, it was determined that this method may not be an adequate formalism to apply to the 
present problem, because convergence of both the ground and excited state vibrational manifolds in $\left(\mathrm{D}_{2} \mathrm{O}_{2}\right.$ is needed. Thus, the original attemp: at such a calculation was abandoned, and further thought will be required to treat the multidimensional dynamics of the water dimer.

\subsection{Conclusion}

The tunneling dynamics of this particular vibration have been well characterized, and this Chapter provides the motivation for searching to higher frequency, so that other vibrations may also be identified and examined. The value of such an accomplishment provides two benefits. First a second vibration, of either the normal or perdeuterated water dimer, will be useful in comparing the multidimensional tunneling dynamics with those presented in this work. Secondly, a continuation of this effort will certainly aid in calculating the IPS for the water dimer, as well as provide a detailed description of the intermolecular forces which govern the tunneling dynamics exhibited by the cluster. The data regarding the isotopomers is also valuable, for each unique species allows for a single tunneling splitting to be probed independently of the others. This will provide information regarding the coupling of each tunneling motion with the highly anharmonic intermolecular vibration. Lastly this effort should be directly coupled to existing theoretical capabilities. Although the initial attempt to simulate the dynamics did not seem feasible with the current level of technology, it may be worthwhile to set up some form of a calculation, for as theoretical efforts develop along with computer technology, such a calculation will be of great value in testing the global usefulness of a water dimer IPS. 
1 For a recent review of the water dimer experiments, see G. T. Fraser, Int. Rev. Phys. Chem. 10, 189 (1991).

2 T. R. Dyke. K. M. Mack, and J. S. Muenter, J. Chem. Phys. 66, 498 (1977).

3 T. R. Dyke, J. Chem. Phys. 66, 492 (1977).

4 J. T. Hougen, J. Mol. Spectrosc. 114, 395 (1985).

5 L. H. Coudert and J. T. Hougen, J. Mol. Spectrosc. 130, 86 (1988).

6 E. Zwart, J. J. ter Meulen, W. Leo Meerts, and L. H. Coudert, J. Mol. Spectrosc. 147, 27 (1991).

7 E. Zwart, J. J. ter Meulen, and W. Leo Meerts, Chem. Phys. Lett. 173, 115 (1990).

8 E. N. Karyakin, G. T. Fraser, and R. D. Suenram, (in Press).

9 L. H. Coudert and J. T. Hougen, J. Mol. Spectrosc..139, 259 (1990).

10 N. Pugliano and R. J. Saykally, J. Chem. Phys. 96, 1832 (1992).

11 R. C. Cohen and R. J. Saykally, J. Phys. Chem. 96, 1024 (1992).

12 M. Inguscio, G. Moruzzi, K. M. Evenson, and D. A. Jennings, J. Appl. Phys. 60(12), Review 161 (1986).

13 This laser frequency was measured in this laboratory.

14 E. E. Haller, Infrared Phys. 25, 257 (1985).

15 P. R. Bunker, Moleculai Symmetry and Spectroscopy (Academic Press Inc., San Diego, CA, 1979).

16 J. A. Odutola and T. R. Dyke, J. Chem. Phys. 72, 5062 (1980).

17 N. Ohashi and J. T. Hougen, J. Mol. Spectrosc. 121, 474 (1987).

18 D. F. Coker and R. O. Watts, J. Phys. Chem. 91, 2513 (1987).

19 B. J. Smith, D. J. Swanton, J. A. Pople, H. F. Schaefer III, and Leo Radom, J. Chem. Phys., 92, 1240 (1990). 
20 M. Quack and M. A. Suhm, Chem. Phys. Lett. 171, 517 (1990).

21 R. D. Suenram, G. T. Fraser, and F. J. Lovas, J. Mol. Spectrosc. 138, 440 (1989).

22 N. Ohashi and J. T. Hougen, J. Mol. Spectrosc. 132, 242 (1988).

23 P. R. Bunker, V. C. Epa, P. Jensen, and A. Karpfen, J. Mol. Spectrosc. 146, 200 (1991) and references within.

24 D. G. Truhlar, Dynamics of Polyatomic van der Waals clusters, ed. K. Janda and N. Halberstadt, (NATO ASI Plenum, New York, NY 1990).

25 P. R. Bunker, T. Carrington, P. C. Gomez, M. D. Marshall, M. Kofranek, H. Lischka, and A, Karpfen, J. Chem. Phys. 91, 5154 (1989).

26 R. C. Cohen and R. J. Saykally, J. Chem. Phys. 95, 7891 (1991).

27 C. A. Schmuttenmaer, R. C. Cohen, J. G. Loessor, R. J. Saykally, J. Chem. Phys. 95. 9 (1991).

28 J. M. Hutson, J. Phys. Chem. 96, 4237 (1992).

29 M. J. Elrod, D. W. Steyert, and R. J. Saykally, J. Chem. Phys. 95, 3182 (1991).

30 R. M. Bentwood, A. J. Barnes, and W. J. Orville-Thomas, J. Mol. Spectrosc. 84, 391 (1980).

31 For example see J. R. Reimers, R. O. Watts, and M. L. Klein, Chem. Phys. 64, 95 (1982).

32 L. H. Coudert, F. J. Lovas, R. D. Suenram, and J. T. Hougen, J. Chem. Phys. 87, 6290 (1987).

33 J. W. I. van Bladel, A. van der Avoird, P. E. S. Wormer, and R. J. Saykally, J. Chem. Phys. 97, 4750 (1992).

34 J. G. Loeser, C. A. Schmuttenmaer, R. C. Cohen, M. J. Elrod, D. W. Steyert, R. J. Saykally, R. E. Bumgarner, and G. A. Blake, J. Chem. Phys. 97, 4727 (1992).

35 J. W. I. van Bladel, A. van der Avoird, and P. E. S. Wormer,J. Phys. Chem. 95, 
5414 (1991).

36 H. Popkie, H. Kistenmacher, and E. Clementi, J. Chem. Phys. 59, 1325 (1973).

37 R. A. Zare, Angular Momentum; Understanding Spatial Aspects in Chemistry and Physics, (John Wiley \& Sons, New York, NY, 1988).

38 The J. G. Loesor Identity. 


\section{Chapter 4}

\section{The Measurement of Vibration-Rotation-Tunneling Dynamics in the Water Trimer- $d_{6}$ Cluster}

\subsection{Introduction}

Despite decades of extensive investigation into the properties of water and its chemical behavior, ${ }^{1}$ there remain important unanswered questions which have potentially significant impact on many chemical and biological problems. For example, the intermolecular forces that exist between water molecules, and are typically oversimplified within the context of current theoretical methods used to study the condensed phases of water (especially with regard to nonpairwise additive interactions, which are poorly characterized through experiments). Although many-body forces are genc-ally considered to be weak relative to the dominant pairwise additive forces, they are clearly important in contributing to the characteristics of the condensed phase. For example, it is well known that pair and higher order distribution functions in liquids extend well beyond the nearest neighbor solvation shell, and that low frequency phonon motions, described by the collective oscillation of a large ensemble of molecules, are also present in the bulk phase. These observations, indicate that short range order exists in liquids over a distance which is larger than the diameter of the first solvation shell. Such interactions of many molecules would be more completely defined by the inclusion of both pairwise and nonpairwise additive interactions. This is particularly important for $\mathrm{H}$-bonded liquids, for the intermolecular energies in the condensed phase are known to substantially deviate from a simple pairwise additive summation. Because of the inadequate description of nonpairwise 
additivity, the treatment of liquid phase phenomena is most commonly accomplished by assuming that pairwise additive forces are solely responsible for the characterization of the solution phase properties. Although this assumption is straight forward to apply, it oversimplifies the bulk by neglecting the weak contributions originating from multibody forces, and this is particularly important for structured liquids, such as water.

A related phenomenon, also of great importance, ${ }^{2}$ is the concerted hydrogen bond tunneling motions that occur in the bulk phases of water and in $\left(\mathrm{H}_{2} \mathrm{O}\right)_{n}$ clusters, for which $\mathrm{n} \geq 2$. By examining the tunneling dynamics in larger water clusters, possible extensions to analogous motions in the condensed phase, such as water librational motions and the concerted rearrangement of hydrogen bonding networks, may develop. It is interesting to note that each of these dynamical considerations occur on similar time scales between 10 $100 \mathrm{ps}^{2}$ as was discussed in Chapter 1. These considerations critical elements in gaining a detailed understanding of the nature of many chemical phenomena, as well as the structure of liquid water itself.

By measuring the low frequency van der Waals intermolecular vibrations in clusters with resolution of rotational and tunneling motions using tunable FIR lasers, and then analyzing the resulting VRT spectra in terms of PI group theory and scattering theory, pair potentials of unprecedented accuracy can be obtained. ${ }^{3}$ Also, by examining the detailed dynamics of clusters, the development of condensed phase dynamics will be enhanced through the careful extension of the gas phase knowledge to the bulk. There is a great deal of work currently available with regard to aqueous processes ( as was presented in Chapter 1), and as discussed in the previous chapter, the water dimer has been the subject of numerous investigations by FIR-VRT spectroscopy and other high resolution spectroscopic techniques. The resulting characterization of the dimer structure and the hydrogen bond tunneling dynamics among the structurally equivalent forms has been an important accomplishment, which has had significant impact in studying condensed phase problems. ${ }^{4}$ However, it is clear that these two extremes must be connected by exploring the properties 
of larger water clusters. This creates the excluing possibility of investigating cooperative (nonpairwise) effects in hydrogen bonding through VRT spectroscopy of the water trimer, and it is this line of thought which motivates the present study.

In this chapter the first detailed experimental results for the cyclic water trimer are presented. Over 100 FIR-VRT transitions were measured for the $d_{6}$ isotopomer in the frequency range of $86.7-92.0 \mathrm{~cm}^{-1}$ with a precision of ca. $1 \mathrm{ppm} .^{5}$ The data are interpreted in terms of a c-type (parallel) VRT band of an oblate symmetric rotor, with rotational constants in good agreement with the cyclic structure predicted from ab initio calculations. 6,7.8.9 Many of the observed rovibrational transitions appear as an evenly spaced quartet of lines which have relative intensities corresponding to those calculated for transitions between VRT states of "F" symmetry in the PI group G96. The structural parameters obtained indicate that each of the monomers behaves both as a donor and as an acceptor, as in the bulk phase of water. Yet possibly the most interesting deduction from the structural analysis is that the cyclic water trimer exists as a transient chiral molecule on a subpicosecond time scale, and the interconversion of the left and right handed stereoisomers occurs via low barrier quantum tunneling motions.

The description of the water trimer will be presented in this chapter as follows. Section 4.2 will describe the particular aspects of the experimental setup used when collecting the water trimer data, as well as the description of an isotopic substitution experiment which was used to unambiguously identify the spectral carrier. In Section 4.3 the fitted rotational constants will be presented and discussed in terms of their structural implications. The spectrum is strongly perturbed by the various tunneling motions, and this will also be discussed. In Section 4.4 the detailed group theoretical analysis, which is found to explain the observed VRT multiplets, is presented. Concluding remarks regarding the proposed transient chirality of the water trimer and future experimental strategies will be contained in Section 4.5. 


\subsection{Experimental/Isotope Experimental Verification}

The Berkeley tunable far-infrared (FIR) laser absorption spectrometer has been thoroughly described in Chapter 2 and the included references, hence only the details pertaining to the water trimer search will be presented here. Four far-infrared laser lines were used in this work: $2588361.8 \mathrm{MHz}\left({ }^{13} \mathrm{CH}_{3} \mathrm{OH}\right) ; 2633900 \mathrm{MHz}\left({ }^{13} \mathrm{CH}_{3} \mathrm{OH}\right)$; $2664085.3 \mathrm{MHz}\left(\mathrm{CH}_{2} \mathrm{DOH}\right)$; and $2714715.1 \mathrm{MHz}\left({ }^{13} \mathrm{CH}_{3} \mathrm{OH}\right)$. By mixing continuously tunable mirrowaves (2-65 GHiz) with the fixed frequency far-IR laser in the 1T12 Schottky barrier diode, tunable FIR ratiation is obtained. The tunable sidebands $\left(v_{\text {sidebands }}=v_{\text {laser }}\right.$ $\left.+/-v_{\text {miciowaves }}\right)$ are separated from the fixed frequency carrier with a Martin-Puplett diplexer. Further separation is accomplished with two 750 line/inch nickel meshes operating as a tunable Fabry-Perot etalon. The entire laser beam path was continuously purged with dry $\mathrm{N}_{2}$ or Ar to permi scannirig in spectral regions in which FIR atmospheric water transitions exist. The radiation enters the molecular beam chamber through a $1 \mathrm{~mm}$ thick polyethylene winduw and is multipassed 10 times through the four inch long $\mathrm{cw}$ plarar expansion in which the $\mathrm{x}$ ater trimer is formed and supersonically cooled to a rotational temperature near $5 \mathrm{~K}$. The highest sensitivity was obtained when a 0.001 " slit width was used. The laser radiation exits the chamber and is detected by the liquid helium cooled Ge:Ga stressed photoconcuctor discussed in Chapter 2, and absorption features are measured as a decrease in detected sideband power. The tunable sidebands are frequency modulated at $50 \mathrm{kHz}$ and absorption signals are demodulated at $2 f$ yielding a minimum detectable fractional absorption of $1 \mathrm{ppm}$. The water trimer absorption signal was sptimized by bubbling argon thrcugh neat $\mathrm{D}_{2} \mathrm{O}$ (99.9\% purity) at a pressure of 900 torr which produces a typical chamber pressure of 300 mtorr. The Ar carrier gas had to be bubbled through the $\mathrm{D}_{2} \mathrm{O}$ liquid, beca.-se it was determined that no water trimer lines were detectable if the carrier gas was simply passed over a reservoir of $\mathrm{D}_{2} \mathrm{O}$.

To verify that the spectral carrier belongs to that of the water trimer an isotopic substitution experiment was conducted with $\mathrm{H}_{2} \mathrm{O} / \mathrm{D}_{2} \mathrm{O}$ mixtures. Assuming that the 
hydrogens in each mixture statistically randomize, and neglecting kinetic isotope effects, the intensity reduction of a given trimer VRT transition due to the equal probability of forming the trimer with protons rather than deuterons follows the simple relationship,

$$
\ln \text { [fractional intensity] }=\mathrm{N} \ln \left[\text { mole fraction of } \mathrm{D}_{2} \mathrm{O}\right. \text {, }
$$

in which $\mathrm{N}$ would be four or six for a cluster containing two and three $\mathrm{D}_{2} \mathrm{O}$ subunits, respectively, and [fractional intensity] is equal to the ratio of the intensity of the transition observed with the mixture versus a pure $\mathrm{D}_{2} \mathrm{O}$ sample. This relation has been shown to give correct results for $\left(D_{2} O\right)_{2}$ transitions (i.e. where $\mathrm{n}$ is equal to four). For this experiment, two identical bubblers were used for trimer isotopic substitution in which one contained a pure $\mathrm{D}_{2} \mathrm{O}$ sample which served as the control vessel. The $\mathrm{D}_{2} \mathrm{O} / \mathrm{H}_{2} \mathrm{O}$ mixture was prepared in a sequential manner, by repeatedly diluting the previous sample contained in the second bubbler, with a measured amount of $\mathrm{H}_{2} \mathrm{O}$. For each mole fraction, a control experiment was conducted from which the fractional intensity ratio was determined. A logarithmic plot of [mole fraction of $\mathrm{D}_{2} \mathrm{O}$ ] versus [fractional intensity] of a representative $\mathrm{R}$ branch transition (with $5 \%$ precision) for five different $\mathrm{D}_{2} \mathrm{O} / \mathrm{H}_{2} \mathrm{O}$ mixtures yields a value of $\mathrm{N}$ equal to $5.5+/-0.4$ at the $90 \%$ confidence limit. These data are are plotted in Figure 4.1. In Figure 4.2, the transition is shown for three different mole fractions of $\mathrm{D}_{2} \mathrm{O} / \mathrm{H}_{2} \mathrm{O}$ equaling 1:0, 0.89:0.11, and $0.85: 0.15$. The scan of the $100 \% \mathrm{D}_{2} \mathrm{O}$ mixture is representative of the quartet structure of the VRT multiplet which will be referred to throughout this chapter.

\subsection{Results}

In Figure 4.3 the observed water trimer spectrum is shown in which the relative intensities are those which have been measured. Each of the reported transitions is split into an evenly spaced quartet and the frequencies of the largest component for each 
Figure 4.1

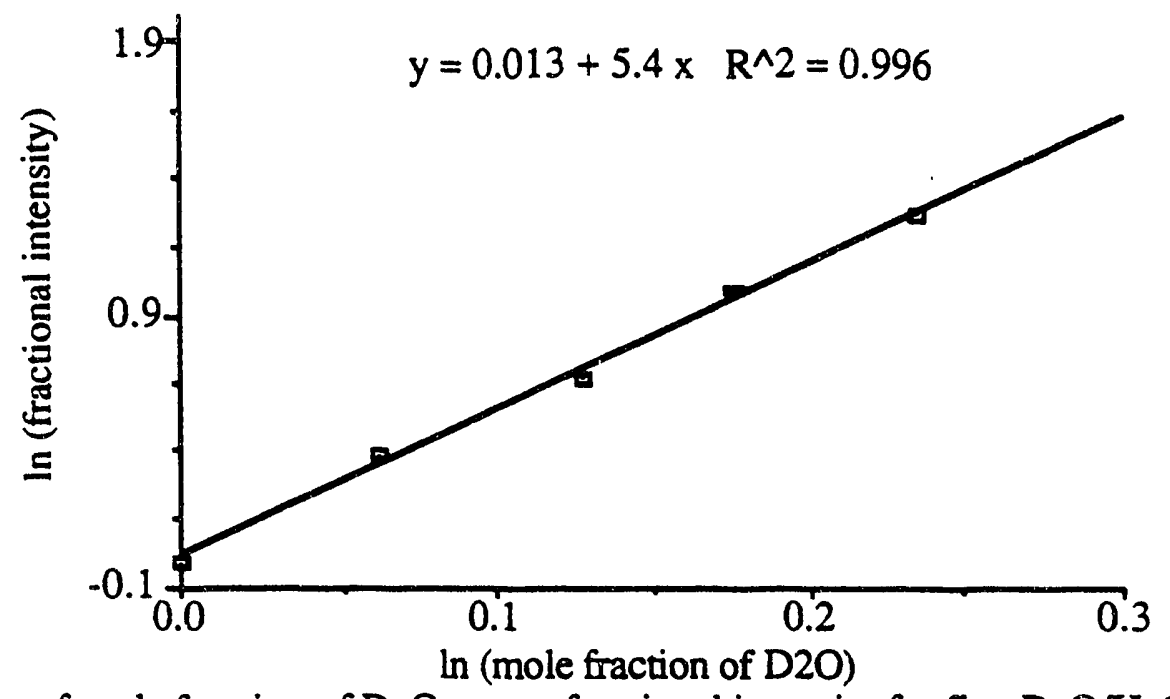

A ln-ln plot of mole fraction of $\mathrm{D}_{2} \mathrm{O}$ versus fractional intensity for five $\mathrm{D}_{2} \mathrm{O} / \mathrm{H}_{2} \mathrm{O}$ mixtures is shown. The slope is determined as 5.4 and the statistical analysis of the data yields an uncertainty of 0.4 at the $90 \%$ confidence limit.

Figure 4.2

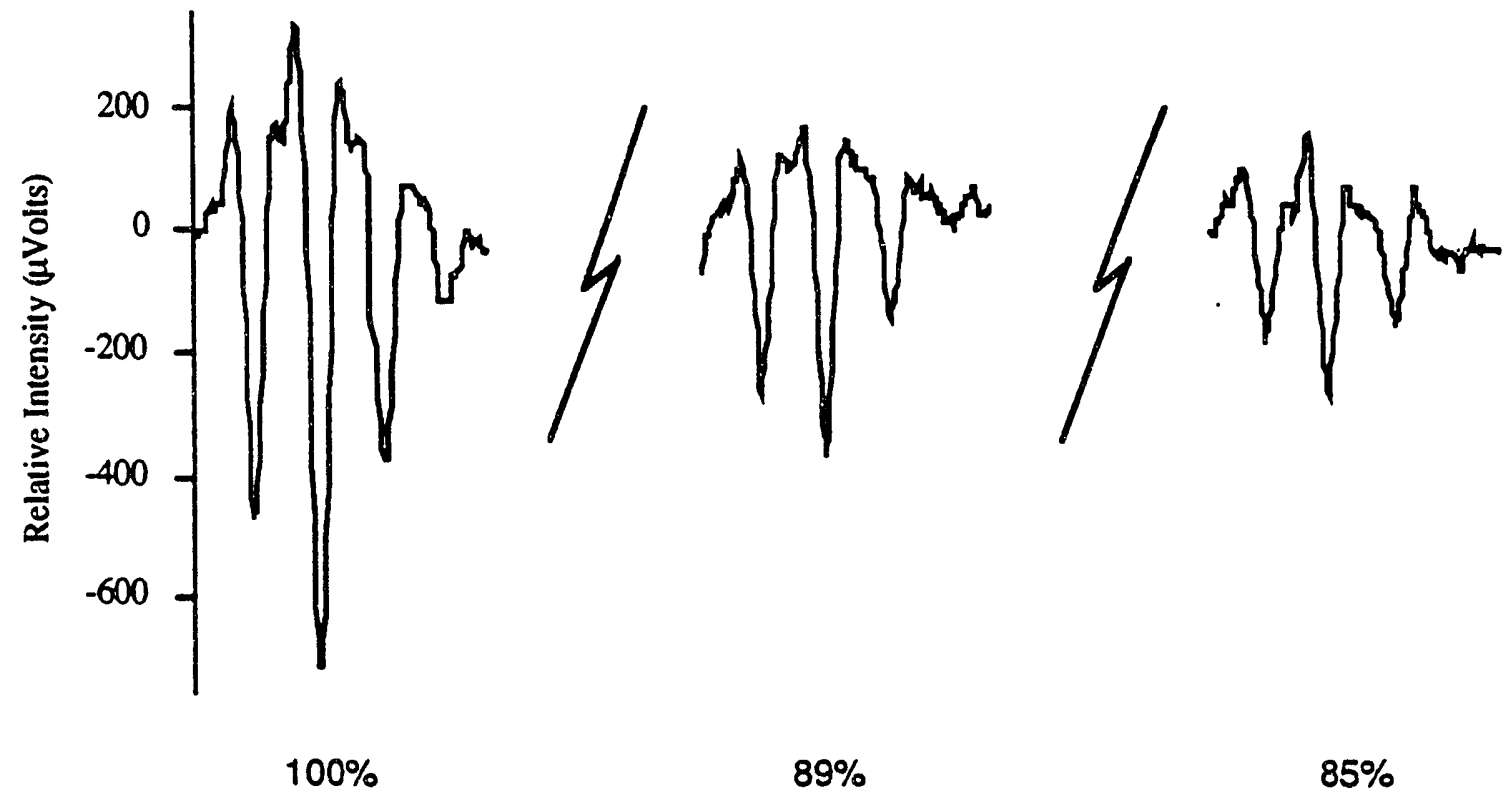

Three different concentrations for the $\mathrm{D}_{2} \mathrm{O} / \mathrm{H}_{2} \mathrm{O}$ isotopic intensity reduction experiments. The $100 \%$ scan represents the quartet feature observed for many of the VRT transitions. The intensity reduction approximately follows the relationship of Equation 4.1 in which the value of $\mathrm{N}$ equals 6 . The $\%$ mole fraction of $\mathrm{D}_{2} \mathrm{O}$ for the scans are listed below each of the VRT features. These concentrations should theoretically yield \% fractional intensities of $50 \%$ and $38 \%$ for the $89 \%$ and $85 \%$ mixtures, respectively, relative to the $100 \% \mathrm{D}_{2} \mathrm{O}$ sample. Each of these scans were taken with a 3 second time constant and background subtraction. Also, substantial effort was needed to keep the $2714 \mathrm{GHz}^{13} \mathrm{CH}_{3} \mathrm{OH}$ laser stable. 
Figure 4.3

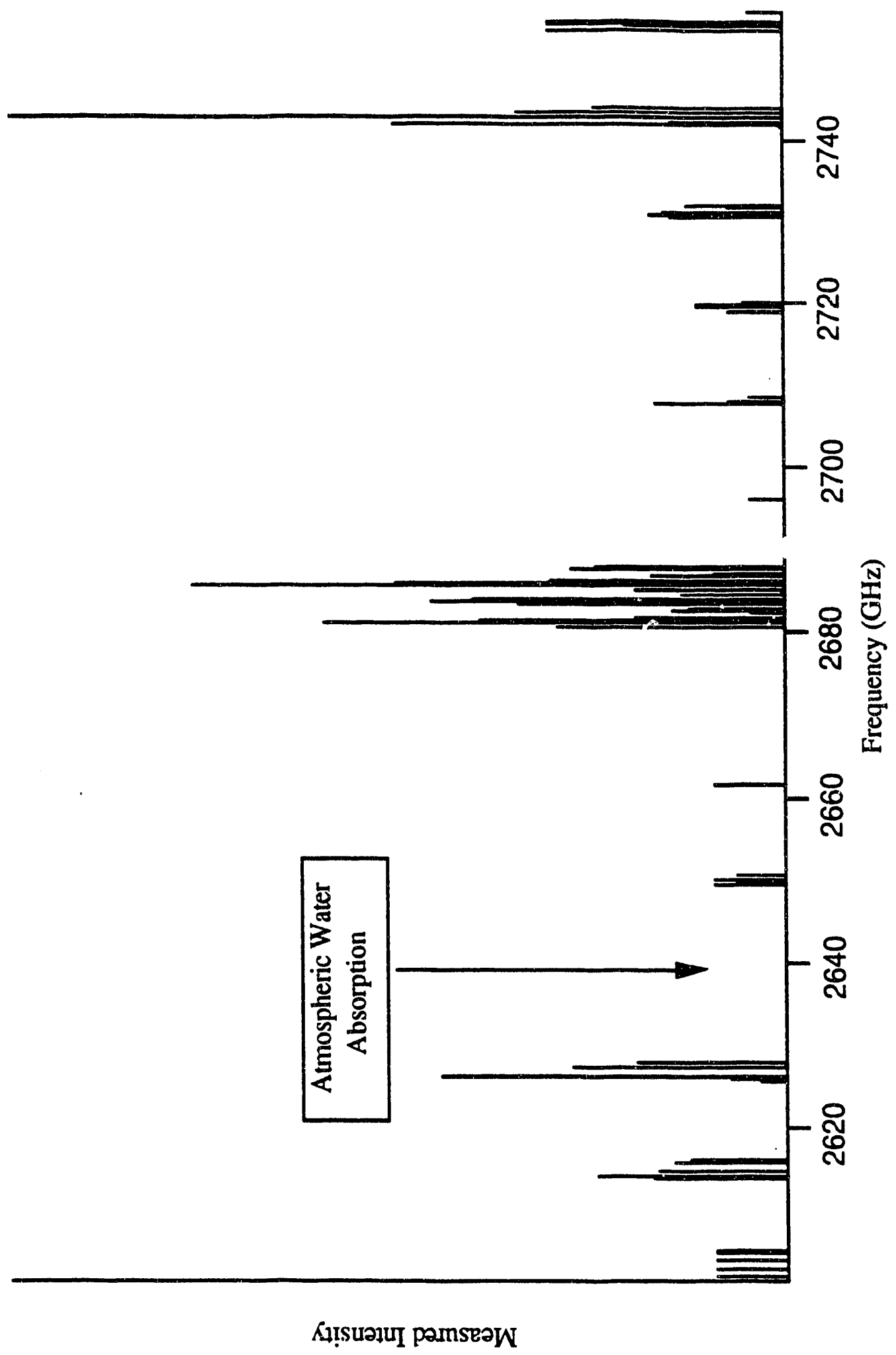

The observed VRT band of the water trimer- $\mathrm{d}_{6}$ is displayed as a stick spectrum. 
multiplet are listed in Table 4.1. The transitions are reported in this manner because the weakest component within the multiplet was not al'ways observable due to sensitivity limitations. The splitting between each of the components of the quartet is ca. $6 \mathrm{MHz}$. This provides a definitive signature for the VRT transitions belonging to this particular intermolecular vibration. The band can be best described as having a contour which can be modeled as a parallel vibrational transition of either a or c type, for a near prolate or oblate asymmetric rotor, respectively. More precisely, a strong $Q$ branch consisting of ca. 60 VRT transitions is found in a very compact spectral region and the relatively weaker $\mathrm{P}$ and $\mathrm{R}$ branches lie to the red and blue, respectively. The $\mathrm{P}$ and $\mathrm{R}$ branches can best be described as groups of transitions among the $\mathrm{K}$ stacks corresponding to a particular value of $\Delta \mathrm{J}$, spaced evenly by $11.5 \mathrm{GHz}$, on average. Also, the number of transitions for a given $\Delta \mathrm{J}=+/-1$ transition follows the relationship of $2 \mathrm{~J}+1$, as is expected for a parallel transition in a near-oblate asymmetric rotor. These two results along with the isotope data, verify that the VRT band describes the cyclic form of the water trimer. The Q branch exhibits several constant patterns, however the spacing between each line (first difference) is ca. $200-400 \mathrm{MHz}$, whereas the second difference for each of these patterns is ca. 1 - 10 $\mathrm{MHz}$. This indicates that the band is strongly perturbed, and this will be further discussed throughout this chapter.

Each of the transition frequencies in Table 4.1 are labeled by the $J$ value. Definitive $\mathrm{K}_{\mathrm{a}}, \mathrm{K}_{\mathrm{c}}$ assignments are not possible for all of the transition frequencies, because the VRT band exhibits strong perturbations in both the upper and lower vibrational states as was determined by the fitting of combination differences in both of these states. This was accomplished by assigning a particular $\mathrm{J}$ to each energy level and then fitting all possible combinations of the $\mathrm{P}$ and $\mathrm{R}$ branch transitions to combination differences for the various $\mathrm{K}_{\mathrm{c}}$ manifolds in each vibrational state. These fits incorporated only the $\mathrm{A}, \mathrm{B}$, and $\mathrm{C}$ rotational constants because it was assumed that if either state was unperturbed the low $\mathrm{J}$ combination differences would be weakly dependent on any of the distortion constants. 
Table 4.1

The observed frequencies of the trimer multiplets reported (in $\mathrm{MHz}$ ).

$\begin{array}{ccccc}\Delta \mathrm{J}=7->6 & \Delta \mathrm{J}=6->5 & \Delta \mathrm{J}=5->4 & \Delta \mathrm{J}=3->2 & \Delta \mathrm{J}=2->1 \\ 2602.2600 & 2613.9565 & 2625.8467 & 2649.6130 & 2661.4952 \\ 2603.1036 & 2614.1614 & 2626.0647 & 2649.8620 & \\ 2604.2138 & 2614.3767 & 2626.2959 & 2650.1280 & \\ 2605.3552 & 2614.4077 & 2626.3726 & 2650.1120 & \\ 2605.0170 & 2614.6013 & 2626.5356 & 2650.7137 & \\ & 2614.8301 & 2627.4490 & & \\ & 2615.8176 & 2627.7214 & & \\ & 2616.0686 & 2627.8262 & & \end{array}$

2681.0442

2681.2959

2681.3904

2681.6204

2681.7334

2681.8538

2681.9443

2682.0697

2682.2047

2682.2611

2682.3994

2682.5555

2682.5781

2682.7183

2682.7346
The Observed Q Branch Multiplets

$\begin{array}{lll}2682.8887 & 2684.3301 & 2686.2332 \\ 2682.9011 & 2684.3879 & 2686.3442 \\ 2683.0610 & 2684.6375 & 2686.3994 \\ 2683.0815 & 2684.6760 & 2686.4751 \\ 2683.1958 & 2685.0017 & 2686.5623 \\ 2683.2449 & 2685.1621 & 2686.5930 \\ 2683.3833 & 2685.2910 & 2686.7151 \\ 2683.4345 & 2685.3665 & 2686.7773 \\ 2683.5000 & 2685.4299 & 2686.9473 \\ 2683.5841 & 2685.4832 & 2686.9801 \\ 2683.7024 & 2685.6697 & 2687.1802 \\ 2683.7991 & 2685.7368 & 2687.4063 \\ 2684.0183 & 2685.8945 & 2687.6294 \\ 2684.0959 & 2685.9868 & 2687.8454 \\ 2684.2491 & 2686.1216 & 2688.0630\end{array}$

\begin{tabular}{|c|c|c|c|c|c|}
\hline $\begin{array}{l}\Delta J=0->1 \\
2696.5410\end{array}$ & $\begin{array}{l}\Delta \mathrm{J}=1 \rightarrow 2 \\
2707.8198 \\
2708.3341 \\
2708.0925\end{array}$ & $\begin{array}{l}\Delta \mathrm{J}=2 \rightarrow>3 \\
2719.1026 \\
2719 \quad 7 \\
2719.6208 \\
2719.7803 \\
2720.1964\end{array}$ & $\begin{array}{l}\Delta J=3->4 \\
2730.3828 \\
2730.6132 \\
2731.1251 \\
2731.1582 \\
2731.6555 \\
2731.7117 \\
2732.0234\end{array}$ & $\begin{array}{l}\Delta J=4->5 \\
2741.4629 \\
2741.6557 \\
2741.8730 \\
2742.1013 \\
2742.3435 \\
2742.4696 \\
2742.5994 \\
2743.2156 \\
2743.3213 \\
2743.5147\end{array}$ & $\begin{array}{l}\Delta J:=5->6 \\
2752.9255 \\
2753.1233 \\
2753.5581 \\
2753.7119 \\
2753.7959 \\
2754.0359 \\
2755.3120\end{array}$ \\
\hline
\end{tabular}


Unfortunately this procedure yielded extremely large residuals for each vibrational state, and an unambiguous assignment of the $\mathrm{K}_{\mathrm{a}}$ and $\mathrm{K}_{\mathrm{c}}$ quantum numbers is not possible with the present data set. The resulting fit of the band is consistent with these observations. Three progressions were fit as the $K_{c}=0$ and 1 subbands. More data has not been included because of the lack of sensitive spectral coverage, and this prohibited the identification of progressions for $K_{c}>1$. The progression which contained the $\Delta J=0->1$ transition was assigned as the $\mathrm{K}_{c}=0$ manifold, whereas the other two were assunied to belong to $\mathrm{K}_{\mathrm{c}}=1$. The only way a reasonable fit could be obtained was with very large values of the DJK distortion constant. Also, since the band structure indicates is parallel transition, the values of the two $\mathrm{C}$ rotational constants are difficult to obtain independently with the amount of information includer in this fit because the parameters are correlated. However, $(\mathrm{A}+\mathrm{B}) / 2$ is well determined for both the ground and excited states. The molecular constants (in $\mathrm{MHz}$ ) resulting from a fit of the three progressions are: $\mathrm{A}^{\prime \prime}=$ $5884.6(74), \mathrm{B}^{\prime \prime}=5702.7(88), \mathrm{C}^{\prime \prime}=2806.3(92), \mathrm{D}^{\prime \prime} \mathrm{JK}=27.4$ (11), $\mathrm{A}^{\prime}=5844.9$ (58), $B^{\prime}=5719.8(73), C^{\prime}=3018(10), D_{J K}^{\prime}=14.71(90)$, and $a$ band origin, $v_{0}=2684979.4$ (29). The uncertainties in these parameters are rather high when compared to those obtained for other near symmetric rotors. Therefore, some comments regarding the fit are noted. First of all, when including other distortion constants such as $D_{\mathrm{J}}$ and $D_{\mathrm{K}}$, no meaningful change in the quality of the fit was observed as indicated by the standard deviation of $8 \mathrm{MHz}$. Since both the ground and excited vibrational states are perturbed, the inclusion of data for larger $\mathrm{K}_{\mathrm{c}}$ was not possible without the extension of progressions in these subbands. Also, the value for the two $\mathrm{DJK}_{\mathrm{JK}}$ distortion constants are extremely large, and this result can be compared with those found for the water dimer. In the original microwave double resonance experiments of Dyke, Mack, and Muenter ${ }^{10}$ the transitions between states of $\mathrm{E}$ symmetry were fit to a standard rigid rotor Hamiltonian with distortion constants. Each of the two sets of transitions between $E$ states were fit for three isotopomers of the water dimer $\left(\left(\mathrm{H}_{2} \mathrm{O}\right)_{2},\left(\mathrm{D}_{2} \mathrm{O}\right)_{2}\right.$, and $\left.\left(\mathrm{H}_{2}{ }^{18} \mathrm{O}\right)_{2}\right)$. Six values for DJK 
resulted and their magnitude ranged from $0.7-4.0 \mathrm{MHz}$. Since this work, these values have been reinterpreted as the result of the donor tunneling shift, and it is clear that the water dimer rotational constants are not entirely representative as structural constants. Therefore, it is presumed that tunneling motions produces the same effect for the water trimer band, and the DJK values do not provide an indication of the molecular structure. However, they indicate that strong dynamical influences are present from the large amplitude tunneling motions.

Even though the fitted rotational constants are not entirely representative of the structural parameters, a calculated structure is presented as an estimate for the equilibrium separation between the three pairs of oxygen atoms within the cluster. The three oxygenoxygen separations are each labeled with a vector ROA.OB in which each vector points from oxygen $A$ to oxygen $B(A, B=1,2,3)$. These three vectors were determined by moving three point masses of $20 \mathrm{amu}$ over a planar grid of spatial points, Luttil the moments of inertia were reproduced. Each set of three points sampled represented the vibrationally averaged position for the center of mass of each monomers. This grid was then scanned for the set of points which best reproduced the fitted rotational constants. Once the center of mass positions were determined for the three monomers, the individual $\mathrm{D}_{2} \mathrm{O}$ molecules were positioned about the center of mass points, and rotated through their corresponding Euler angles (relative to the space fixed axis of the planar grid) to reproduce the H-bonded angles of the structural water trimer of Reference 5. The angles for these bonds are defined as $\mathrm{DO}_{1 O 2}=28^{\circ}, \mathrm{DO}_{2} \mathrm{O}_{3}=30^{\circ}$, and $\mathrm{DO}_{102}=27^{\circ}$, and were assumed to lie in the plane of the ring. Finally, each of the out-of-plane deuterium atoms are fixed in a plane which is perpendicular to the six-membered ring. This calculated structure for the water trimer-d6 is shown in Figure 4.4 from above the plane of the cyclic ring, and is indicative of the vibrationally averaged cluster. To clarify the structure of Figure 4.4 , it is noted that the deuterium on $\mathrm{O} 1$ is below the plane of the ring, whereas those residing on $\mathrm{O}_{2}$ and $\mathrm{O} 3$ extend above this plane. The structure calculated in this manner, reproduces the ground 
Figure 4.4

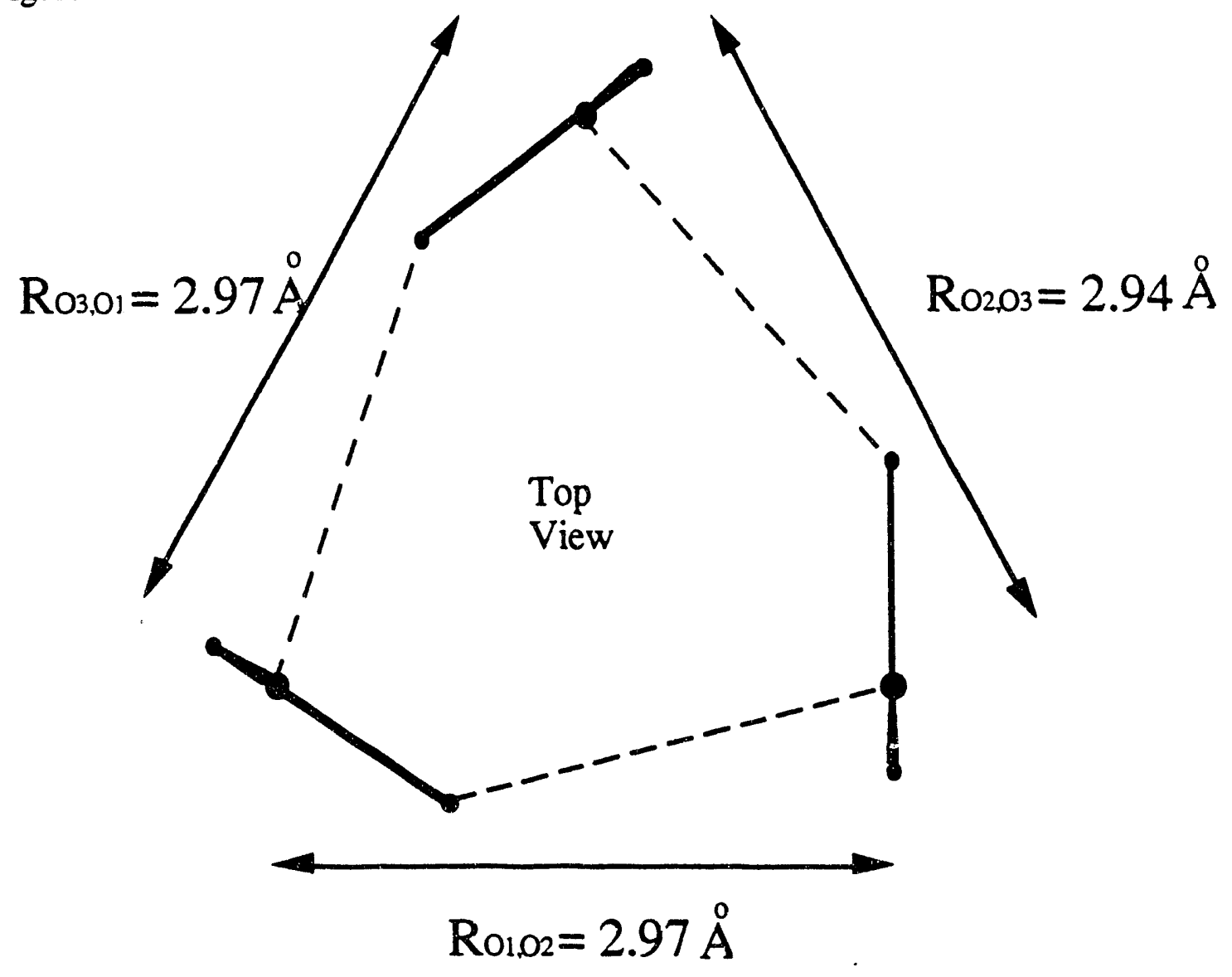

The calculated structure for the water trimer-d6 as obtained from the fitted rotational constants.

scate rotational constants presented in this work with an error of $+1-0.03 \AA$ when $R O 1,02$ $=2.97 \AA$, RO2, $03=2.94 \AA$, and $\mathrm{RO} 3,01=2.97 \AA$. These vibrationally averaged values for $\mathrm{RC}_{\mathrm{A}}, \mathrm{OB}$ can be compared to the oxygen-oxygen separation in the water dimer, which has been determined to be $2.976 \AA$.

\subsection{VRT Multiplet Interpretation}

\subsubsection{The Formulation of the Full Molecular Symmetry Group}

As was described for the water dimer in Chapter 3, tunneling splittings of the rovibrational energy levels appear when identical particles are able to pass through IPS 
barriers. Such tunneling motions permit the extensive sampling of symmetrically equivalent forms of the molecule. In the limit for which the IPS barriers are high, each of the equivalent minima posses degenerate rovibrational eigenvalues, and no tunneling splittings are observable. Thus, the only trajectory between adjacent minima is one which takes the molecule over the large IPS barriers, and this process usually requires the breaking of chemical bonds. For the limit in which the barriers are small, the tunneling of identical particles through low energy pathways can easily be accomplished. This causes the mixing of the zero order rovibrational energy levels, located in the various equivalent minima, and observable spectral splittings result. This interaction requires the necessity for additional symmetry labels to characterize the resultant VRT energy levels diagram. To account for the full symmetry of the system, permutation-inversion (PI) group theory is adopted to classify the VRT eigenstates, as was demonstrated in the previous chapter. In weakly bound van der Waals clusters, such as the water trimer, large amplitude tunneling motions correlate with hindered rotations of each monomer, and are facile motions which depend on the anisotropic nature of low barriers on the multidimensional IPS. The most facile of the tunneling motions produce the largest tunneling splittings, which may be resolvable with narrow band laser systems. Knowledge of the spectral splitting pattern helps to determine plausible tunneling pathways, and gives information regarding the anisotropy on the IPS along each of the various tunneling coordinates. Therefore, a correlation diagram for these splittings must be constructed, for it is a valuable resource toward the understanding of the dynamics in these weakly bound clusters. Such a diagram describes the effects of each tunneling motion on the rovibrational energy levels, and is used to rationalize the observed spectral splitting patterns of a particular cluster. Thus, a correlation diagram for the water trimer will be proposed to explain the observed tunneling splitting pattern for the measurer? VRT band located near $89.6 \mathrm{~cm}^{-1}$.

The construction of the trimer correlation diagram will begin by assuming the molecule exhibits no tunneling; in other words the IPS barriers are extremely large. The 
most stable equilibrium structure for the water trimer has been determined through high level $a b$ initio theory6-9 to be a cyclic ring in which the oxygen-oxygen separations constitute the sides of an isosceles triangle. For this minimum energy structure, each water subunit serves as both an $\mathrm{H}$-bond donor and an $\mathrm{H}$-bond acceptor. Two such structures which adhere to the above description have been found to exist. In one of these, two of the remaining three free hydrogens point above the plane of the ring whereas the third is directed below, and in the other form all three of the free hydrogens reside on the same side of the ring. In Figure 4.5 an illustration of these two structures, labeled as trans and cis, is presented. In this figure, each atom is uniquely labeled so as to make them distinguishable

Figure 4.5

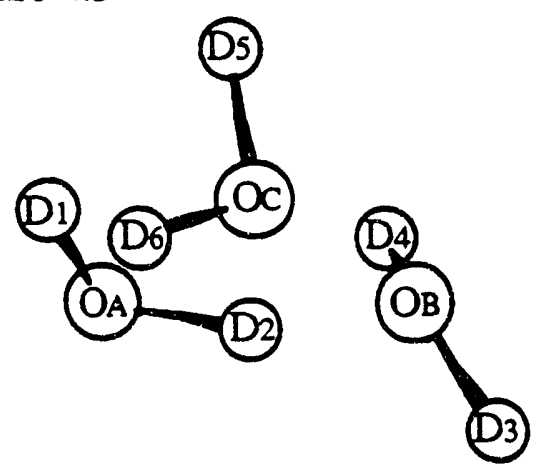

Trans-form

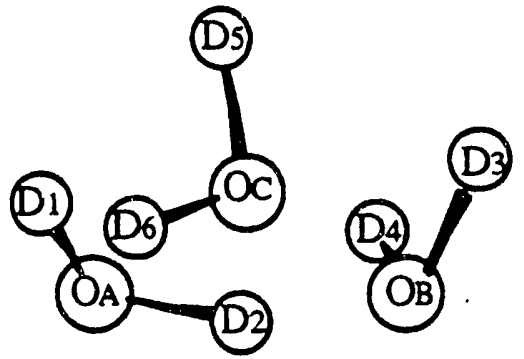

Cis-form

The two nearly isoenergetic forms of the water trimer- $\mathrm{d}_{6}$ van der Waals cluster labeled as trans and cis.

from each other. The only point group symmetry operation for the trans isomer is the trivial identity, whereas the cis form contains the identity and a $C_{3}$ axis of rotation. This defines the symmetry groups for the two molecules as $C_{1}$ and $C_{3}$, respectively. An interesting aspect of these two structures is that their mirror images are not superimposable on their original forms because the $\mathrm{O}-\mathrm{H}-\mathrm{O} \mathrm{H}$-bond is asymmetric, ${ }^{11}$ therefore, they are 
both chiral rings in the structural limit.

At this point the individual tunneling motions will be introduced separately. Through the tunneling of the identical particles, symmetrically equivalent forms of the cluster may be accessed, and this introduces the additional permutation-inversion symmetry operations which are applicable to the cluster. The complete nuclear permutation-inversion (CNPI) group for this cluster contains 8640 operations (3! x 6! x 2) when simply considering the complete permutation and inversion of all identical particles ( 3 oxygen atoms and 6 protons). However, the breaking of water $\mathrm{O}-\mathrm{H}$ bonds is not considered for this problem and the relevant molecular symmetry group reduces to an order of

$$
\left[S_{3}\right]\left[S_{2}\right]^{3} \mathrm{I}=96 .
$$

In Equation 4.2, $\left[\mathrm{S}_{3}\right]$ represents the permutation group which allows for he interchange of each of the oxygen atoms, $\left[S_{2}\right]$ represents the permutation group for the two hydrogen atoms on a particular water subunit, and I is the inversion of all particles through the cluster center of mass. The group $\left[S_{2}\right]$ is cubed because of the presence of the three individual water subunits within the cluster. The subgroups $S_{3}$ and $S_{2}$ are made up of the following operations,

$$
S_{3}=\{E,(A B),(B C),(C A),(A B C),(A C B)\},
$$

and for each water monomers,

$$
S_{2}=\{E,(12)\}, \text { or }\{E,(34)\} \text {, or }\{E,(56)\} .
$$

From these two subgroups, the full molecular symmetry group of the water trimer can be generated in terms of the Generalized Character Cycle Index (GCCI) approach which has been discussed by Balasubramaniun and Dyke. ${ }^{12}$ This treatment automatically generates the form for each of the 96 PI operations of the water trimer, and each of these operations are listed in Table 4.2. A GCCI term of the form $x_{n}{ }^{m}$, corresponds to " $m$ " cyclic 
Table 4.2.

The permutation group $\mathrm{G}_{48}$ representative of the water trimer created by the direct product of $\left[\mathrm{S}_{3}\right]$ and $\left[\mathrm{S}_{2}\right]^{3}$; inversion is not included. This group is isomorphic with the $\mathrm{O}_{h}$ point group. The full molecular symmetry group of $\mathrm{G}_{96}$ is made up of each of these elements and this set of operations multiplied by $E^{*}$.

Permutation Operation

$$
\text { GCCI Terma Conjugacy of }
$$

E

(12), (34), (56)

$\begin{array}{ll}\mathrm{x}_{1}{ }^{6} & 1 \\ \mathrm{x}_{1}{ }^{4} \mathrm{x}_{2} & 3 \\ \mathrm{x}_{1}{ }^{2} \mathrm{x}_{2}{ }^{2} & 3 \\ \mathrm{x}_{2}{ }^{3} & 1 \\ \mathrm{x}_{1}{ }^{2} \mathrm{x}_{2}{ }^{2} & 6 \\ \mathrm{x}_{1}{ }^{2} \mathrm{x}_{4} & 6\end{array}$

$\mathrm{AB}(1324)(56), \mathrm{AB}(1423)(56), \mathrm{BC}(3546)(12)$,

$\mathrm{BC}(3645)(12), \mathrm{AC}(1625)(34), \mathrm{AC}(1526)(34)$

$x_{2} x_{4} 6$

$\mathrm{AB}(13)(24)(56), \mathrm{AB}(14)(23)(56), \mathrm{BC}(12)(36)(45), \quad \times 2^{3}$ $\mathrm{BC}(12)(35)(46), \mathrm{AC}(15)(26)(34), \mathrm{AC}(16)(25)(34)$

$A B C(146)(235), A B C(135)(246)$, $A B C(145)(246), A B C(136)(245)$,

$\mathrm{x}^{2}-8$

$\mathrm{ACB}(153)(264), \mathrm{ACB}(164)(253)$,

$\mathrm{ACB}(154)(263), \mathrm{ACB}(163)(254)$

6

$A B C(146235), A B C(136245)$,

$x_{6}$

8

$A B C(135246), A B C(145236)$,

$A C B(164253), A C B(153264)$,

$\mathrm{ACB}(154263), \mathrm{ACB}(163254)$

a GCCI will serve as a label to describe the form of a particular class of elements in the group $\mathrm{G}_{48}$. 
permutations of index " $n$ ". The table is organized in terms of similar GCCI terms, and this grouping automatically defines the conjugacy of each class of operations. Each of these operations converts an initial framework of the trimer into a symmetrically equivalent form, since the cluster Hamiltonian is invariant to the permutation of identical particles or the inversion of the particles through the cluster center of mass. Some of these operations also interconvert right and left handed enantiomers of the cluster, and these will be considered when appropriate.

In generating the correlation diagram, only the details of the trans structure will be considered in detail, because this is found to be the most stable form of the cluster, and involves the more complicated group theoretical treatment. The time averaged position for each of the out-of-plane hydrogens for the $C_{1}$ structure, places them approximately in the plane of the ring. This is intuitive because the barrier to this transition state is extremely low, thus permitting the facile motion of the hydrogens from above to below the plane of the ring. This "librational" type of tunneling motion is equivalent to a simple rotation of each water subunit about its hydrogen bond so that the out-of-plane hydrogen flips from above, to below the plane, and this motion will be referred to as the "flipping" tunncling coordinate. ${ }^{13}$ The calculated height of the barrier through which this motion proceeds is ca. 40 and $30 \mathrm{~cm}^{-1}$ for $\left(\mathrm{D}_{2} \mathrm{O}\right)_{3}$ from References 5 and 9, respectively. Thus, the large amplitude "flipping" tunneling motion is considered to be very feasible due to the presence of the small barrier. The symmetry operations which take an initial framework into frameworks resulting from a "flipping" tunneling event are defined by the subgroup of operations

$$
\begin{gathered}
\{\mathrm{E},(\mathrm{ACB})(153)(264),(\mathrm{ABC})(135)(246), \\
\left.\mathrm{E}^{*},(\mathrm{ACB})(153)(264) *,(\mathrm{ABC})(135)(246) *\right\},
\end{gathered}
$$

for which $E$ is the identity, (ACB) etc. is defined as a cyclic permutation of identical 
nuclei ${ }^{14}$ such that $A$ moves to position $C, C$ moves to $B$, and $B$ moves to $A$, and the (*) represents the inversion of all particles through the center of mass of the cluster. These six operations represent the action of tunneling between symmetrically equivalent structures of the trimer, and to a irst approximation the tunneling pathway may be defined as the simple rotation about the $\mathrm{H}$-bond as mentioned earlier. An interesting aspect of these operations is noticed when realizing that that three of them $\left(E^{*},(\mathrm{ACB})(153)(264) *,(\mathrm{ABC})(135)(246) *\right)$ interconvert right and left handed enantiomeric forms, whereas the remaining three (E, $(A C B)(153)(264),(A B C)(135)(246))$ simply preserve the handedness. Figure 4.6, taken from Reference 4 represents tunneling of the deuteron labeled 5 in the left handed structure along a pathway which follows a rotation about the $\mathrm{O}_{A} \cdots \mathrm{D}_{5}-\mathrm{O}_{C}$ hydrogen bond. This operation, oefined in the figure caption and in Reference 10 as $(A C B)(153)(264) *$, is one of the six operations in the $C_{3 h}(M)$ molecular symmetry group, and it interconverts the right and left handed enantiomers of the cluster. The result of each of the six operations on the initial framework of the cluster are shown in Figure 4.7.

In the limit wherein the flipping motion is the only means of tunneling, the molecular symmetry group will coirelate to a molecular point group which is isomorphic to $\mathrm{C}_{3 \mathrm{~h}}$ and is thus, called $\mathrm{C}_{3 \mathrm{~h}}(\mathrm{M})$. As a result of the "flipping" tunneling motions, each of the IJ, K> rotational energy levels will split into six VRT states, which have the symmetries $A^{\prime}, A^{\prime \prime}, E^{\prime}$, and $E^{\prime \prime}$ of the irreducible representations of $C_{3 h}(M)$. The correlation diagram shown in Figure 4.8 illustrates the degeneracy breaking of the $\mathrm{IJ} \mathrm{K}>$ due to the flipping tunneling metion. In this tunneling limit a large splitting is assumed between the various VRT components because of the extremely low barrier for this motion (ca.30-40 cm-1 ). As a result, four unique VRT sublevels will arise. These sublevels will be separated in energy by the large flipping tunneling splitting. Such a low barrier motion is consistent with the unlikely possibility of the presence of a permanent dipole moment along the $\mathrm{C}$ inertial axis, because the individual dipoles of the water subunits are constantly fluctuating. This is further corroborated by the molecular beam electric field deflection experiments of Dyke 
Figure 4.6 Caption

The chirality of the water trimer- $\mathrm{d}_{6}$ cluster is illustrated by displaying the cyclic ring structure along with its mirror image. The handedness of an enantiomer is defined by projecting the dipole moment of each subunit into the plane of the cyclic ring. Each projection is depicted beneath the rings and the right handed enantiomer is defined so that when following the dipole projections in a right handed sense, the resulting orientation is aligned with the positive Z-axis. The identical nuclei within the cluster are labeled so as to make them distinguishable from one another. An example of an operation which permutes the identical nuclei is written as (ACB)(153)(264)*, for which (ACB) etc. is defined in Reference 13 as a cyclic permutation of identical nuclei such that $A$ moves to position $C, C$ moves to $B$, and $B$ moves to $A$, and the $*$ represents the inversion of all particles through the center of mass of the cluster. The pathway for this operation is equivalent to "flipping" $D_{5}$ from above to below the plane of the cyclic ring by rotating about the $O_{A} \cdots D_{5}-O_{B}$ hydrogen bond. The simple rotations about the two molecule fixed axes, orient the cluster so that its mirror image can easily be noticed. Tunneling through this low barrier pathway is an example of how the left and right handed enantiomers of the cluster are interconverted. 
Figure 4.6

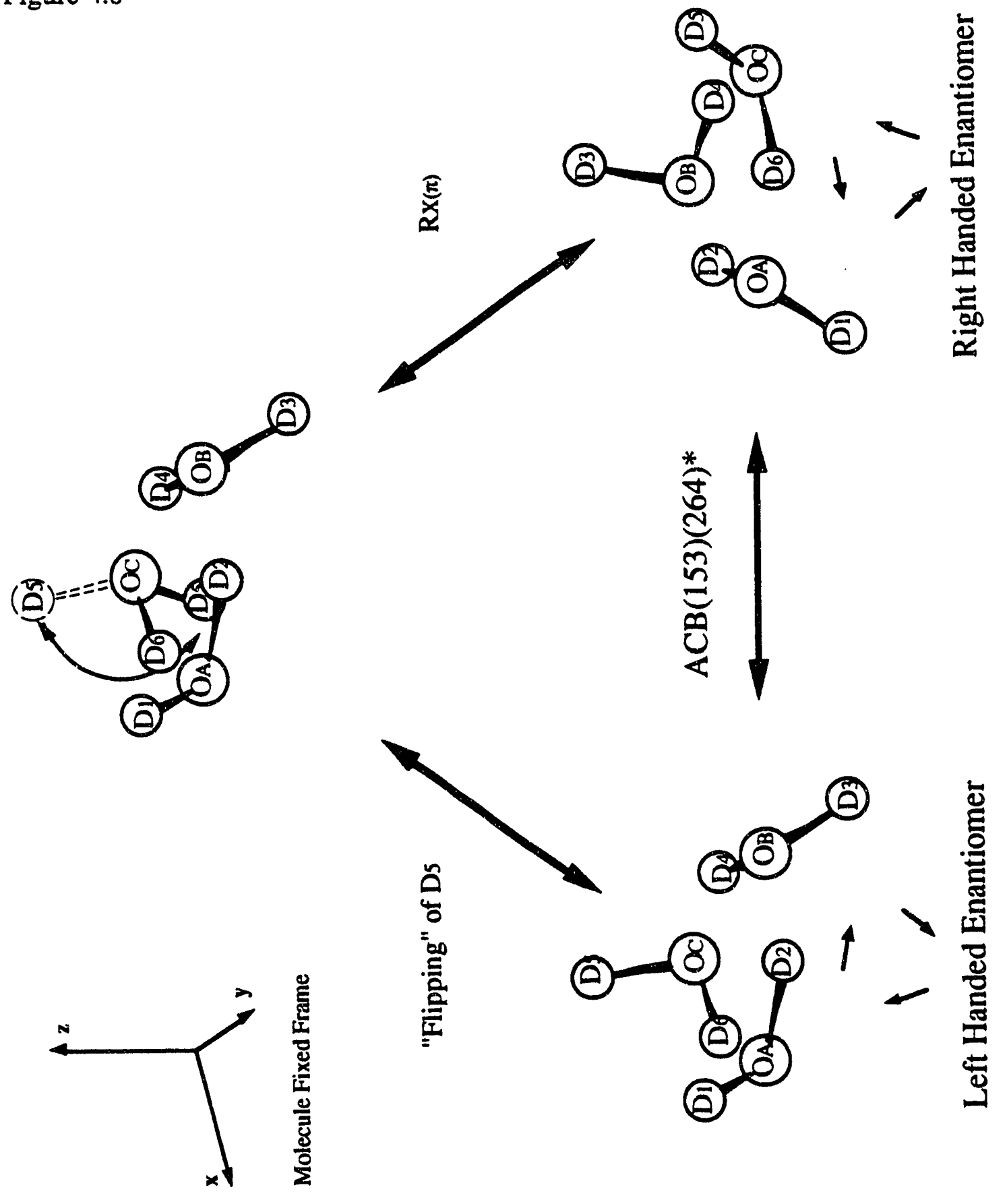


Figure 4.7

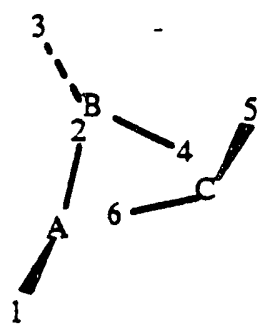

$\mathrm{E}$

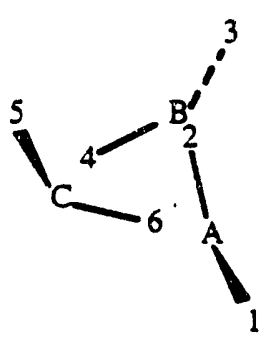

$E^{*}$

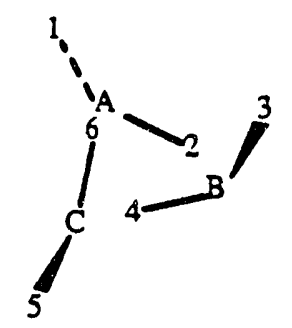

$\mathrm{ABC}(135)(246)$

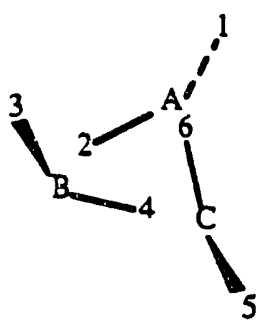

$\mathrm{ABC}(135)(246)^{*}$

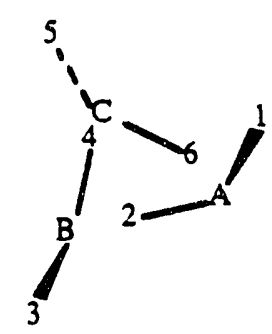

$\mathrm{ACB}(153)(264)$

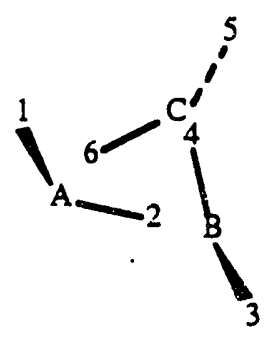

$\operatorname{ACB}(153)(264) *$

The six symmetrically equivalent forms of the water trimer generated by the permutationinversion operations which correspond to the flipping of the out of plane hydrogens from above to below the plane of the ring. These six operations form a subgroup which is isomorphic to $\mathrm{C}_{3 \mathrm{~h}}(\mathrm{M})$. 
Figure 48

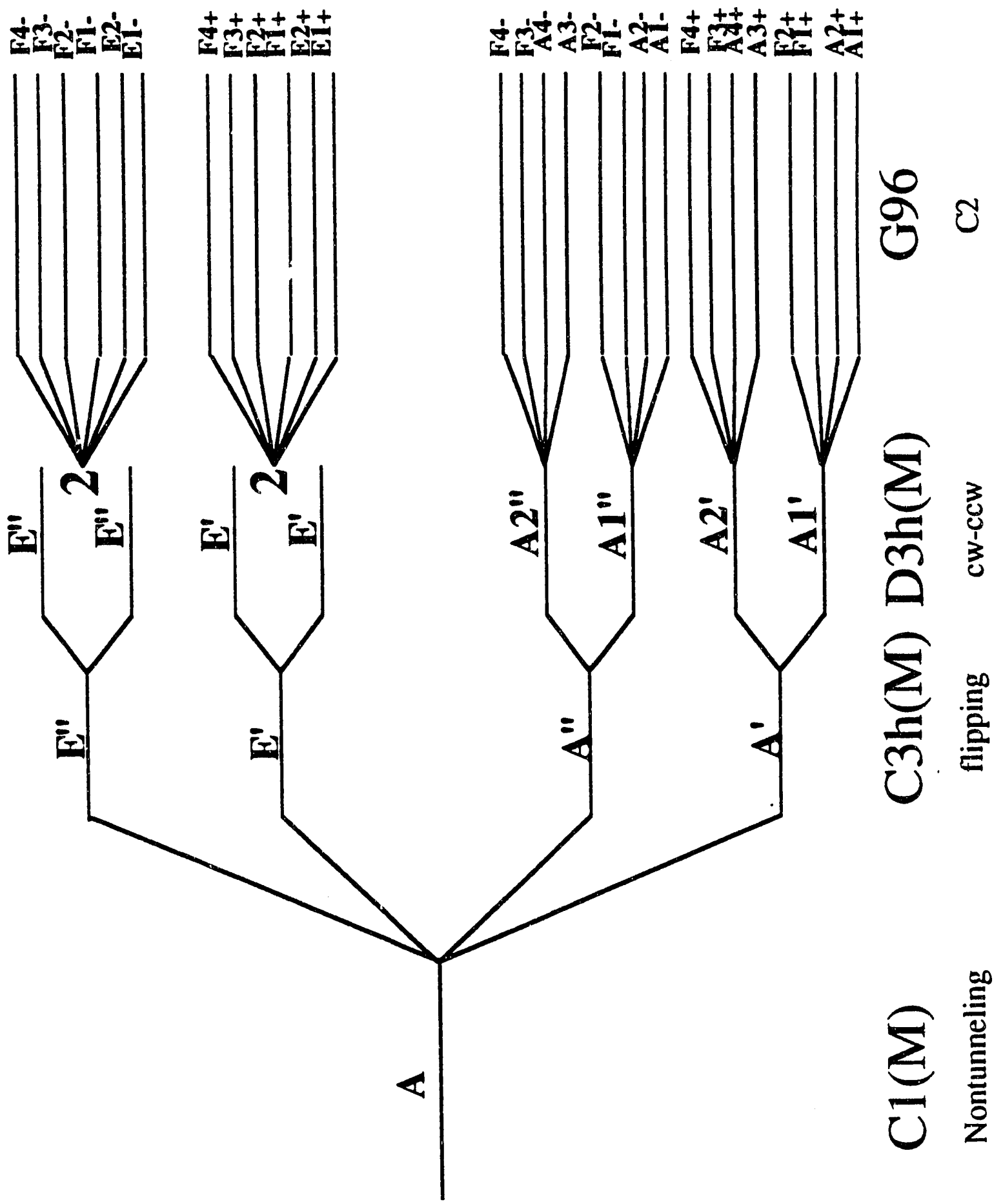

The complete correlation diagram for the water trimer from the rigid, nontunneling limit to G96. Each of the E states in the $D_{3 h}(M)$ limit correlate to the six $G_{96}$ states, and thus the number " 2 " is placed in front of these to complete the correlation. Sequential application of Equations 4.7 and 4.8 are used to transform a subgroup to its larger homomorphic group. 
and Muenter. ${ }^{15}$ This extremely facile motion would render microwave spectroscopy nearly impossible.

Another possible low barrier motion is one which reverses the sense of the $\mathrm{H}$ bonding from clockwise to counterclockwise and will be referred to as the "cw-ccw" motion. The operations representing these motions are,

$$
\begin{gathered}
\{(\mathrm{AB})(13)(24),(\mathrm{AC})(15)(26),(\mathrm{BC})(35)(46) \\
\left.(\mathrm{AB})(13)(24)^{*},(\mathrm{AC})(15)(26) *,(\mathrm{BC})(35)(46) *\right\}
\end{gathered}
$$

A simple tunneling pathway is difficult to imagine for this motion, however it most certainly would involve a $180^{\circ}$ rotation of each water monomer about its corresponding C inertial axis which is perpendicular to the plane of each subunit. Presumably this would occur along a concerted reorientational pathway. Since the breaking of the simple H-bond network is required for this motion, the barrier would necessarily be high relative to the flipping tunneling motion, thus producing a small spectral splitting. By taking the direct product of the "flipping" and "cw-ccw" operations, a PI group which is isomorphic with the point group $D_{3 h}$ is constructed, and is therefore called $D_{3 h}(M)$. The correlation between the VRT states of $C_{3 h}(M)$ to those of $D_{3 h}(M)$ can be determined with the following two general expressions,

$$
\begin{gathered}
\Gamma_{\alpha}=\sum_{i=1}^{h} a_{i}^{\alpha} \Gamma_{i}, \\
a_{i}=\frac{1}{h} \sum_{R=1}^{h} \chi^{\Gamma_{\alpha}\left[H_{R}\right] \chi^{\Gamma_{i}}\left[H_{R}\right]^{*} .}
\end{gathered}
$$

In these two equations, $\Gamma_{\alpha}$ and $\Gamma_{i}$ represent the irreducible representations of the $D_{3 h}(M)$ and $C_{3 h}(M)$ groups, respectively, $h$ is the order of the subgroup ( $h$ of $C_{3 h}(M)=6$ ), and $\chi^{\Gamma \propto\left[\mathrm{H}_{\mathrm{R}}\right] \text { and } \chi^{\Gamma i}\left[\mathrm{H}_{\mathrm{R}}\right] \text { are the characters for each of the irreducible representations for each }}$ 
of the elements $H_{R}$ in their respective groups. When applying these expressions it can be determined that the $A^{\prime}$ and $A^{\prime \prime}$ states of $C_{3 h}(M)$ split into $A_{1} / A_{2}^{\prime}$ and $A_{1} " / A_{2}$ " states in $D_{3 h}(M)$, respectively, whereas the $E^{\prime}$ and $E^{\prime \prime}$ states of $C_{3 h}(M)$ transform as $2 E^{\prime}$ and $2 E^{\prime \prime}$ in $D_{3 h}(M)$. The effects of this tunneling motion on the VRT energy level diagram are illustrated in Figure 4.8.

The last tunneling motion to be considered is postulated to correspond to a rotation of each water subunit about its $C_{2}$ axis (B inertial axis). This motion must also occur through the concerted destruction and reformation of the H-bonded network along its tunneling pathway. More precisely, the effect of this tunneling motion would exchange the positions of the two deuterons for each water subunit, thus generating another nonsuperimposable but symmetrically equivalent water trimer. This coordinate will be referred to as " $\mathrm{C}_{2}$ " tunneling, and is described by the following six permutation-inversion operations,

$$
\left\{(12),(34),(56),(12)^{*},(34)^{*},(56)^{*}\right\} \text {. }
$$

If the "flipping", "cw-ccw", and " $\mathrm{C}_{2}$ " tunneling motions were all to occur on a time scale which would produce resolvable splittings, conclusive evidence would then exist for the interconversion between the 96 symmetrically equivalent forms of the trimer. These symmetrically equivalent structures are graphically illustrated in Table 4.3 for the $\mathrm{G}_{96}$ molecular symmetry group. In this Table an operation is applied to the original structure to obtain the final labeled form. Only the 48 structures which may be determined by the operations defined by the product group $\left[S_{3}\right] \times\left[S_{2}\right]^{3}$ are included. To obtain the remaining 48 structures, each labeled form in Table 4.3 would need to be multiplied by $E^{*}$. All of the 96 tunneling motions serve as a pathway which interconverts one labeled form of the trimer into an equivalent labeled form. In this tunneling limit, all of the 96 symmetrically equivalent minima on the IPS are accessible through the proposed tunneling motions. 
Table 4.3

The 48 labeled forms for the cyclic water trimer which arise from the product of $\left[\mathrm{S}_{3}\right] \mathrm{x}$ $\left[\mathrm{S}_{2}\right]^{3}$. To obtain the remaining 48 labeled forms, the inversion operation should be applied to each of the structures shown below.
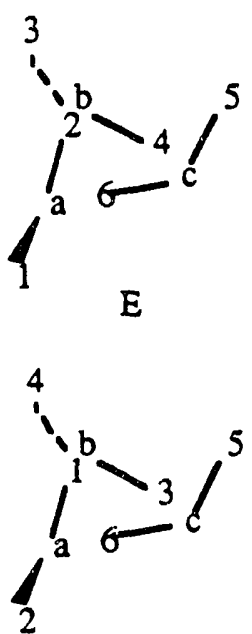

$(12)(34)$

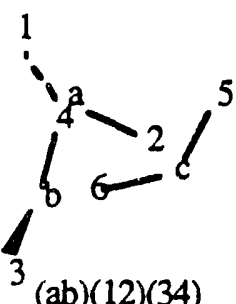

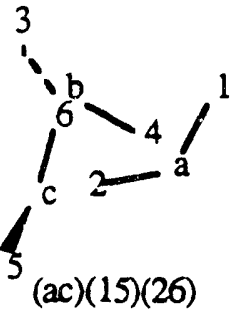<smiles>SP1OP2OCC12S</smiles>

$(b c)(3645)$

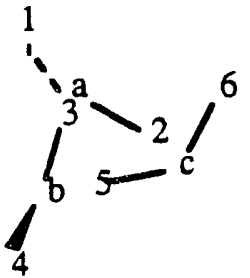

$(\mathrm{ab})(56)(1324)$

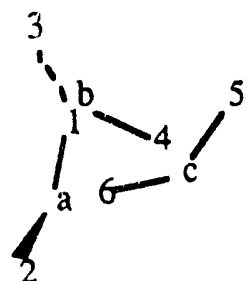

(12)

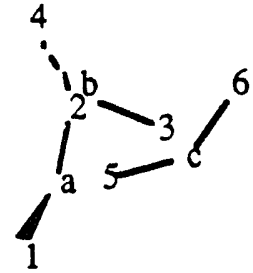

$(34)(56)$
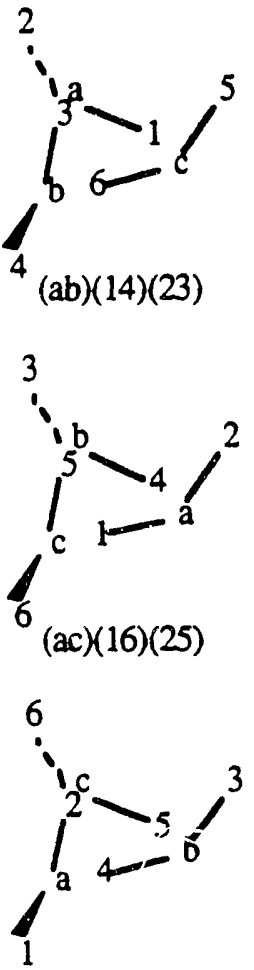

$(\mathrm{bc})(3546)$

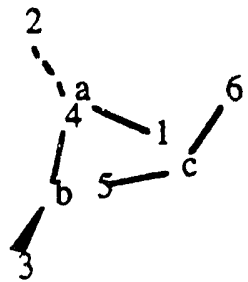

$(a b)(56)(1423)$

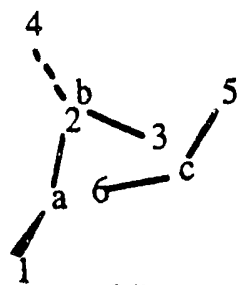

(34)

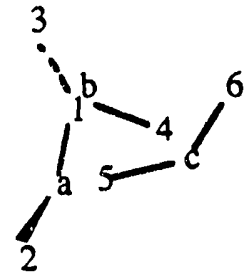

(12)(56)
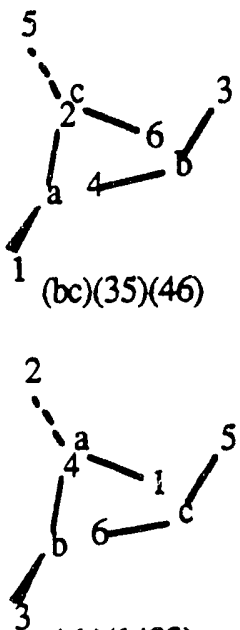

(ab)(1423)

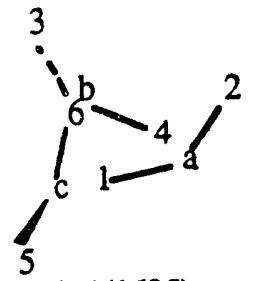

(ac)(1625)

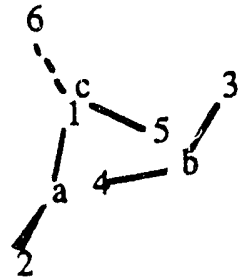

(bc)(12)(3546)<smiles>C[13CH][13CH][13CH][13CH3]</smiles>

(56)

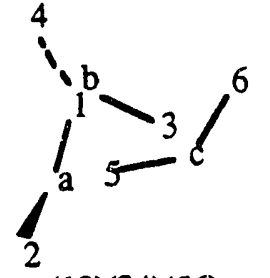

$(12)(34)(56)$
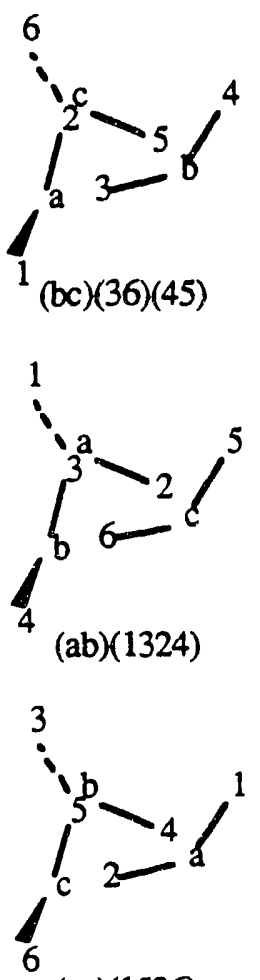

(ac)(1526)

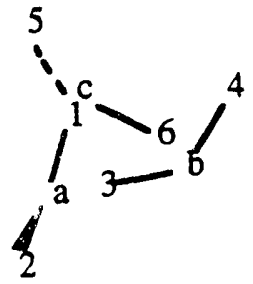

(bc)(12)(3645) 


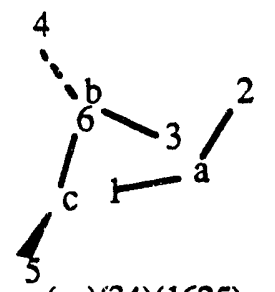

(ac)(34)(1625)

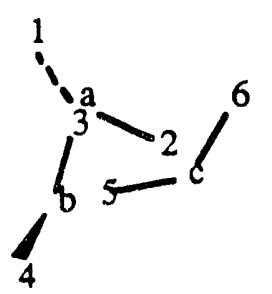

$(a b)(14)(23)(56)$
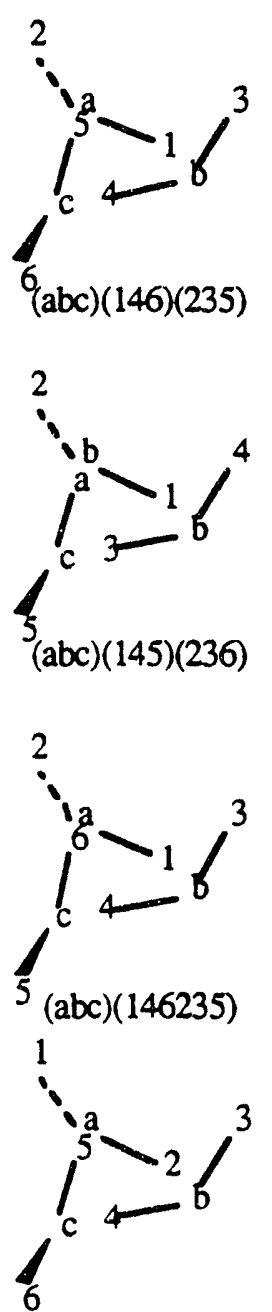

$(a b c)(135246)$
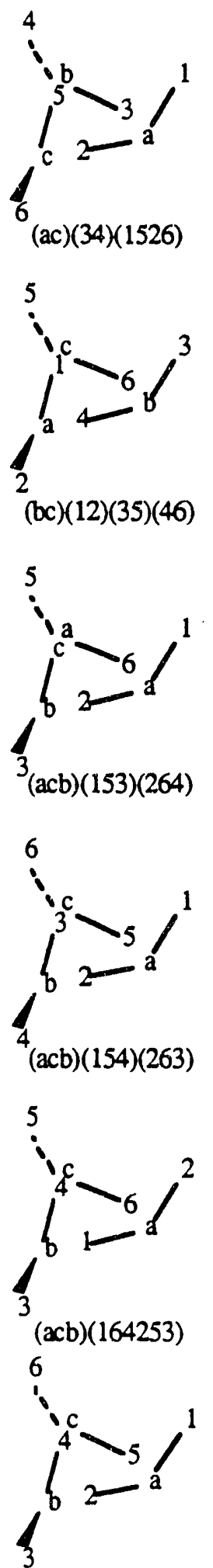

(acb)(154263)

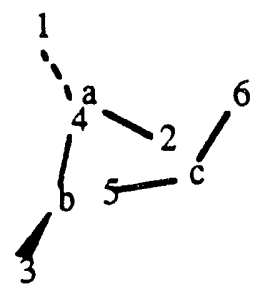

$(a b)(13)(24)(56)$

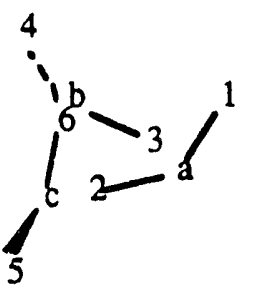

$(a c)(15)(26)(34)$
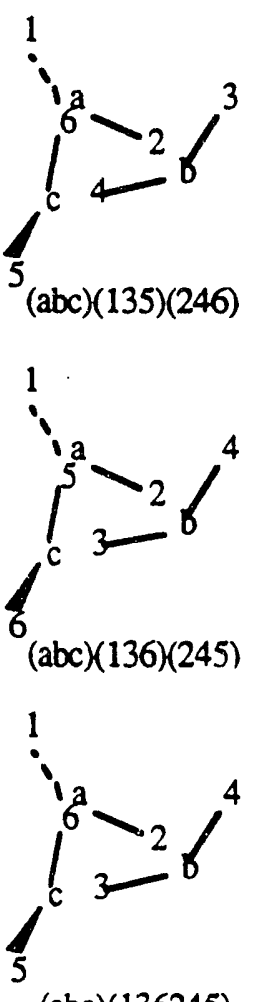

$(a b c)(136245)$

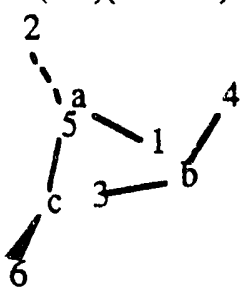

$(a b c)(145236)$

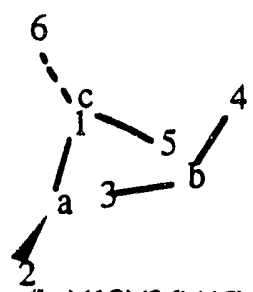

$(b c)(12)(36)(45)$

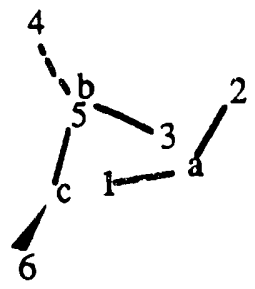

$(\mathrm{ac})(16)(25)(34)$
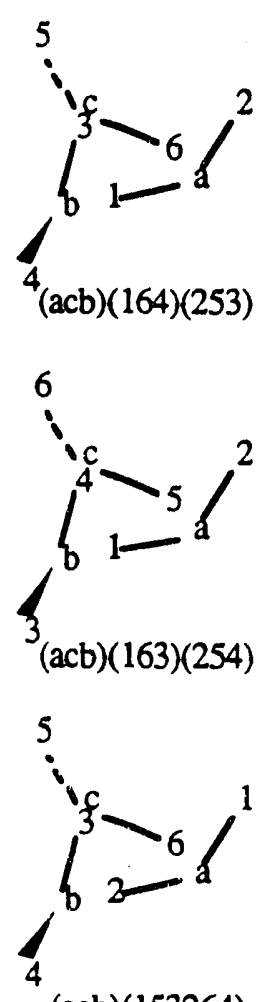

(acb)(153264)

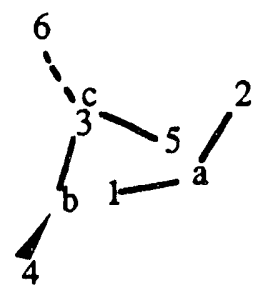

(acb)(163254) 
Furthermore, 48 operations convert a right handed form of the trimer to the left handed form through the low barrier tunneling motions, and 48 preserve the handedness of the cluster. In this situation, the appropriate molecular symmetry groun for the water trimer is G96, which is equal to the group created by the direct product of $C_{i}(M)$ with $O_{h}(M)$. This is the largest group possible for the cyclic water trimer in the limit wherein the chemical bonds of each monomer remain intact.. The character table for $\mathrm{G}_{96}$ can be easily constructed by multiplying the character tables for the point groups $\mathrm{O}_{\mathrm{h}}$ and $\mathrm{C}_{\mathrm{i}}$ together, and the result of this is shown in Table 4.4.16 From the character tables of $G_{96}$ and $D_{3 h}$ the correlation between the twu groups may be accomplished by using the relationships of Equations (4.7) and (4.8). The correlation from $D_{3 h}(M)$ to $G_{96}$ introduces additional splittings of the VRT levels so that a complete breaking of the rovibrational degeneracy occurs for each $\mathrm{IJ}, \mathrm{K}_{\mathrm{a}}, \mathrm{K}_{\mathrm{c}}>$ state, and this tunneling limit is shown in Figure 4.8. The correlation shows that VRT states having $A_{1}{ }^{\prime}, A_{2}{ }^{\prime}, A_{1}$ ", $A_{2}$ " symmetry in $D_{3 h}(M)$ will each split into two A states and two $F$ states, whereas states of E' and E" symmetry in $D_{3 h}(M)$, will each split into two $E$ states and another set of $F$ states. The splitting in the $D_{3 h}(M)$ and $G_{96}$ limits are shown to be much smaller than that which correlates to the $\mathrm{C}_{3 \mathrm{~h}}(\mathrm{M})$ limit, because these tunneling coordinates require the breaking of the $\mathrm{H}$-bonds, and therefore, they are required to pass through large IPS barriers when compared to the "flipping" motion. As determined in Reference 12, the nuclear spin weights for the irreducible representations of $\mathrm{G}_{96}$ are reproduced in Table 4.5 for both the normal and perdeuterated isotopomers. Finally the electric dipole allowed selection rule of $\Gamma_{\mathrm{i}^{+}}<->\Gamma_{\mathrm{i}}$,

When considering the rotational constants for the trimer, and the fact that each tunneling sublevel in the $C_{3 h}(M)$ limit correlates to states of $F$ symmetry, it is not surprising that the observed band is strongly perturbed. As discussed in the previous chapter, the selection rules for a Coriolis perturbation permit the interaction of VRT states between $\mathrm{K}_{\mathrm{a}}$ ' manifolds which are off diagonal by $+/-1$, diagonal in the $\mathrm{J}$ quantum number,

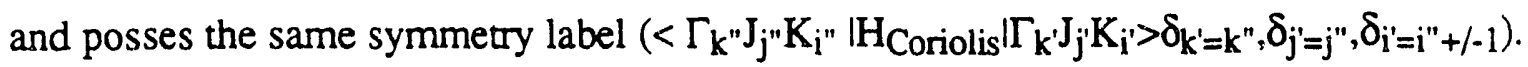


Table 4.4

The character table for $\mathrm{O}_{\mathrm{h}}(\mathrm{M})$ point group. The operations for $\mathrm{G}_{48}$ are defined in Table 4.2 and the class conjugacy is reported below each of the operations. To obtain the $\mathrm{G}_{96}$ character table, the character tables for the $\mathrm{O}_{\mathrm{h}}(\mathrm{M})$ group should be multiplied by that for $\mathrm{C}_{\mathrm{i}}(\mathrm{M})$, as is shown by the equation below.

\begin{tabular}{l|cccccccccc}
$\mathrm{O}_{\mathrm{h}}(\mathrm{M})$ & $\mathrm{x}_{1}{ }^{6}$ & $\mathrm{x}_{2}{ }^{3}$ & $\mathrm{x}_{1}{ }^{4} \mathrm{x}_{2}$ & $\mathrm{x}_{1}{ }^{2} \mathrm{x}_{2}{ }^{2}$ & $\mathrm{x}_{1}{ }^{2} \mathrm{x}_{2}{ }^{2}$ & $\mathrm{x}_{2} \mathrm{x}_{4}$ & $\mathrm{x}_{1}{ }^{2} \mathrm{x}_{4}$ & $\mathrm{x}_{2}{ }^{3}$ & $\mathrm{x}_{3}{ }^{2}$ & $\mathrm{x}_{6}$ \\
\hline $\mathrm{A}_{1}$ & 1 & 1 & 3 & 3 & 6 & 6 & 6 & 6 & 8 & 8 \\
$\mathrm{~A}_{2}$ & 1 & 1 & 1 & 1 & 1 & 1 & 1 & 1 & 1 & 1 \\
$\mathrm{E}_{1}$ & 2 & 2 & 2 & 2 & 0 & 0 & 0 & 0 & -1 & -1 \\
$\mathrm{~A}_{3}$ & 1 & -1 & -1 & 1 & 1 & 1 & -1 & -1 & 1 & -1 \\
$\mathrm{~A}_{4}$ & 1 & -1 & -1 & 1 & -1 & -1 & 1 & 1 & 1 & -1 \\
$\mathrm{E}_{2}$ & 2 & -2 & -2 & 2 & 0 & 0 & 0 & 0 & -1 & $:$ \\
$\mathrm{F}_{1}$ & 3 & -3 & 1 & -1 & 1 & -1 & 1 & -1 & 0 & 0 \\
$\mathrm{~F}_{2}$ & 3 & -3 & 1 & -1 & -1 & 1 & -1 & 1 & 0 & 0 \\
$\mathrm{~F}_{3}$ & 3 & 3 & -1 & -1 & 1 & -1 & -1 & 1 & 0 & 0 \\
$\mathrm{~F}_{4}$ & 3 & 3 & -1 & -1 & -1 & 1 & 1 & -1 & 0 & 0 \\
\hline
\end{tabular}

\begin{tabular}{c|cccc}
$\mathrm{C}_{\mathrm{i}}(\mathrm{M})$ & $\mathrm{E}$ & $\mathrm{E}^{*}$ & & \\
\hline $\mathrm{A}^{+}$ & 1 & 1 & $\mathrm{X}$ & $\left\{\chi \mathrm{O}_{\mathrm{h}}(\mathrm{M})\right\}=\left\{\chi \mathrm{G}_{96}\right\}$ \\
$\mathrm{A}^{-}$ & -1 & -1 & & \\
\hline
\end{tabular}


indicates that transitions occur between states differing only by the parity label.

Table 4.5

Nuclear Spin weights for $\left(\mathrm{H}_{2} \mathrm{O}\right)_{3}$ and $\left(\mathrm{D}_{2} \mathrm{O}\right)_{3}$.

\begin{tabular}{lll|lll}
\hline$\Gamma_{\mathrm{i}}$ & $\left(\mathrm{H}_{2} \mathrm{O}\right)_{3}$ & $\left(\mathrm{D}_{2} \mathrm{O}\right)_{3}$ & $\Gamma_{\mathrm{i}}$ & $\left(\mathrm{H}_{2} \mathrm{O}\right)_{3}$ & $\left(\mathrm{D}_{2} \mathrm{O}\right)_{3}$ \\
\hline $\mathrm{A}_{1}$ & 1 & 56 & $\mathrm{E}_{2}$ & 8 & 8 \\
$\mathrm{~A}_{2}$ & 0 & 20 & $\mathrm{~F}_{1}$ & 3 & 63 \\
$\mathrm{~A}_{3}$ & 10 & 10 & $\mathrm{~F}_{2}$ & 0 & 45 \\
$\mathrm{~A}_{4}$ & 1 & 1 & $\mathrm{~F}_{3}$ & 6 & 36 \\
$\mathrm{E}_{1}$ & 0 & 70 & $\mathrm{~F}_{4}$ & 3 & 18 \\
\hline
\end{tabular}

Thus, since the top rotational constant $C$, is rather small, and each rotational energy level correlates to many states of $F$ symmetry, the interaction between $F$ states of the different $K_{c}$ manifolds is very likely. This congested energy level diagram will therefore, satisfy the above matrix element conditions, and the perturbations in the water trimer spectrum will be extremely complicated. It is also possible that these perturbations are actually variations ir. tunneling splittings with the $\mathrm{K}_{c}$ quantum number. This kind of variation was observer in the previous chapter for the water dimer. If the $\mathrm{K}_{\mathrm{a}}=0 \rightarrow 0$ and $\mathrm{K}_{\mathrm{a}}=0 \rightarrow 1$ subbands for $82.6 \mathrm{~cm}^{-1}$ vibration of the water dimer were fit to a rigid rotor Hamiltonian without the inclusion of the tunneling splitting parameters, very large values for the distortion constants would he needed to obtain reasonable residuals. So when considering the strong possibility for Coriolis perturbations, with the lack of a detailed, $\mathrm{K}_{\mathrm{C}}$ dependent model of the tunneling splittings, the poor fit of the data with only structural constants must suffice in the present analysis.

Finally, it should be noted that for the cis- structure of the trimer, the correlation 
diagram would be need to be modified. This molecule has a $C_{3}$ axis of rotation, therefore, the molecular symmetry group is $C_{3}$, and states of $A$ and $E$ symmetry would be present. The pure rotational symmetry would be such that $A$ states would correspond to values of $\mathrm{K}_{\mathrm{c}}$ which are multiples of 3 , and all other $\mathrm{K}_{\mathrm{c}}$ manifolds $(1,2,4,5$, etc.) would posses $\mathrm{E}$ symmetry. If this structure existed, the correlation to $\mathrm{G}_{96}$ would proceed through the following equation,

$$
\begin{gathered}
A=A_{1}+A_{2}+A_{3}+A_{4}+F_{1}+F_{2}+F_{3}+F_{4}, \\
E=E_{1}+E_{2}+F_{1}+F_{2}+F_{3}+F_{4} .
\end{gathered}
$$

The $G_{96}$ full molecular symmetry group of for the water trimer structure would be isomorphic with the $\mathrm{O}_{h}$ point group. At this time, there is no way of distinguishing between the two structures.

\subsubsection{Interpretation of the Observed Multiplet Structure and the Vibrational Assignment}

The symmetrical quartet patterns observed in the VRT spectra are interpreted as transitions among the $\mathrm{F}$ states, because the relative intensity ratio can best be described by the nuclear spin ratios of $45,63,36$, and 18 as defined in Table 4.5. Since the observation of quartet structure indicates that the slower " $\mathrm{C}_{2}$ " and "cw-ccw" tunneling motions are manifested in the molecule, and since the identification of the F states is reasonably well established, the appropriate symmetry grotp for the water trimer must be $\mathrm{G}_{96}$. Because the F states arise from a tunneling motion which must involve a pathway requiring the breaking of the hydrogen bonds in the trimer, the barriers for these motions are presumed to be fairly high. The small splittings (ca. $6 \mathrm{MHz}$ ) among these VRT energy components are indicative of the change in cunneling splitting between the ground and excited vibrational states.expected, and do not provide direct evidence that the actual splitting is small. The small change in the splitting indicates that this tunneling coordinate is not strongly affected by the intermolecular vibrational motion. However, when considering that the water dimer 
intermolecular vibrational band (presented in the previous chapter) exhibits large changes in the tunneling splittings upon vibrational excitation, it seems likely that the splitting is in fact small for both vibrational states. This small tunneling splitting raises an interesting question regarding the ground state tunneling. The water trimer may not exhibit a spectrally resolvable tunneling motion in $v=0$, which would make the order of the appropriate symmetry group smaller in this state $\left(G_{12}=D_{3 h}(M)\right.$ or even $\left.G_{6}=C_{3 h}(M)\right)$. If this were the case, the $6 \mathrm{MHz}$ tunneling splitting would correspond to the excited state energy separation of the F states.

The rovibrational band of quartets is consistent with a transition origin 1 ting from one of the components (A or E states) of the flipping tunneling splitting described in the $\mathrm{C}_{3 \mathrm{~h}}(\mathrm{M})$ limit. In this limit, it is probable that the VRT band observed at $89.6 \mathrm{~cm}^{-1}$ correlates with a "flipping" type of $\backslash$ urational motion. This motion would certainly exhibit a large dipole derivative along the top axis, and would result in a c-type band, as observed. The identification of the exact $C_{3 h}(M)$ VRT state from which the vibrational transition originates, cannot yet be accomplished because all four irreducible representations in this group correlate to $F$ states in the symmetry group $G 96$, and the accompanying $A$ or $E$ states have not yet been assigned. This vibrational assignment is also consistent with the most recent ab initio predictic is for the water trimer. Table 4.5 lists the predicted vibrational frequencies for this cluster along with the normal isotopomer between $0-200 \mathrm{~cm}^{-1}$, and the calculated band intensities for each. ${ }^{9}$ The DZP/SCF calculations were quoted ${ }^{9}$ as being the most reliable fo: molecular systems which possess large amplitude, highly anharmonic motions. The predicted vibrational frequency for the $\left(\mathrm{D}_{2} \mathrm{O}\right)_{3}$ at 98,118 , and $153 \mathrm{~cm}^{-1}$ all correspond to modes which involve the flipping of the out of plane hydrogens. These modes can be distinguished from the $149 \mathrm{~cm}^{-1}$ vibrational state from their relative integrated band intensities. The three flipping vibrations are all predicted with the DZP?SCF basis set to be strong absorptions relative to the $149 \mathrm{~cm}^{-1}$ band, which is assigned as a planar ring deformation mode. When scaling the perdeuterated water trimer frequency of $98 \mathrm{~cm}^{-1}$ by 
the standard correction factor for these calculations of 0.91 , a value $89.2 \mathrm{~cm}^{-1}$ results. This is in excellent agreement with the observed VRT band located near $89.6 \mathrm{~cm}^{-1}$, thus the vibration is assigned as this corresponding to this flipping mode.

Table 4.5

Predicted harmoniz frequencies (in $\mathrm{cm}^{-1}$ ) for vibrational transitions in the FIR spectral region for the water trimer cluster taken from Reference 15. The band intensities (in $\mathrm{km} / \mathrm{mol}$ ) are shown in parentheses.

\begin{tabular}{llllll}
\hline $\mathrm{DZP} / \mathrm{SCF}$ & \multicolumn{3}{l}{$\mathrm{TZ2P} / \mathrm{SCF}$} & \multicolumn{3}{c}{$\mathrm{DZP} / \mathrm{CISD}$} \\
\hline$\left(\mathrm{H}_{2} \mathrm{O}\right)_{3}$ & $\left(\mathrm{D}_{2} \mathrm{O}\right)_{3}$ & $\left(\mathrm{H}_{2} \mathrm{O}\right)_{3}$ & $\left(\mathrm{D}_{2} \mathrm{O}\right)_{3}$ & $\left(\mathrm{H}_{2} \mathrm{O}\right)_{3}$ & $\left(\mathrm{D}_{2} \mathrm{O}\right)_{3}$ \\
\hline $133(110)$ & 98 & $136(60)$ & 101 & 165 & 121 \\
$154(86)$ & 118 & $145(81)$ & 118 & 180 & 137 \\
$162(8)$ & 149 & $148(7)$ & 136 & 188 & 172 \\
$169(138)$ & 153 & $167(172)$ & 141 & 196 & 176 \\
$194(8)$ & 163 & $179(7)$ & 156 & 218 & 184 \\
$222(53)$ & 186 & $214(48)$ & 172 & 249 & 208 \\
\hline
\end{tabular}

The assigned VRT band and the frequencies for the other calculated bands raise an interesting question regarding the other flipping tunneling sublevels. Since the barrier to the flipping motion is very low, the magnitude of the corresponding tunneling splitting in the $\mathrm{C}_{3 \mathrm{~h}}(\mathrm{M})$ limit is completely unimaginable. Each of these rovibrational transitions in the predicted excited state flipping vibrations may individually split into the correlation diagram of Figure 4.8. However, the flipping tunneling splitting could be so large, that the predicted vibrational bands may correlate to the iridividual tunneling sublevels of $\mathrm{C}_{3 \mathrm{~h}}(\mathrm{M})$ subgroup. These two VRT limits are best understood in terms of the magnitude of the matrix eiements responsibie for each of the vibrationai and filpping motions in the cluster. 
For the first case, the heirarchy of matrix elements would place the energy contribution of the vibrational Hamiltonian above that of the flipping tunneling motion, and this is

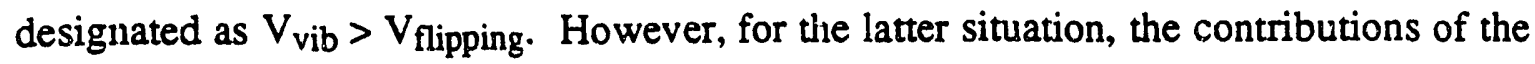
two matrix elements to the energy of the cluster could be nearly equal, or possibly even reversed. These two limits are diagramed in Figure 4.9. Certainly, the rigorous selection rules for $\mathrm{G}_{96}$ will apply, and so this must be included when determining the possible vibrational transitions. In both limits, only the lowest energy subcomponent would have population in the $\mathrm{F}$ states, since thermal relaxation may occur in the molecular expansion between states of F symmetry. So until the next vibration is observed it is not clear which overall picture is more appropriate.

\subsection{Conclusion}

The first observation of this water trimer VRT band reported here, should motivate the study of complex tunneling motions in larger water clusters which may contribute to water-water interactions in the liquid phase. With this study, the importance of these considerations is only beginning to become clear, and much more work is needed to adequately characterize this these types of problems. The tunneling dynamics in the trimer are extremely complex, and to identify all of the VRT components will be very challenging, because initial efforts will have to rely on the relative intensity ratios of transitions which may not involve such distinguishing patterns. However, this is very important because it will test the group theoretical formalism outlined above. An equally important experiment to conduct would be to search for higher frequency vibrational bands belonging to this cluster. The DZP/SCF calculation of Reference 9 predicts that such an absorption should be measured at approximately $107.4 \mathrm{~cm}^{-1}$ after multiplying the reported value by 0.91 . Therefore, the next vibrational band of the water trimer- $d_{6}$ cluster, which has a predicted intensity comparable to that of the $89 \mathrm{~cm}^{-1}$ band, should absorb at a frequency of $118 \times 0.9$ $=106 \mathrm{~cm}^{-1}$. When examining Taule 2.1 in Chapter 2 , it is realized that the $96 \mu \mathrm{m} \mathrm{CH} 3 \mathrm{OH}_{3}$ 
Figure 4.9

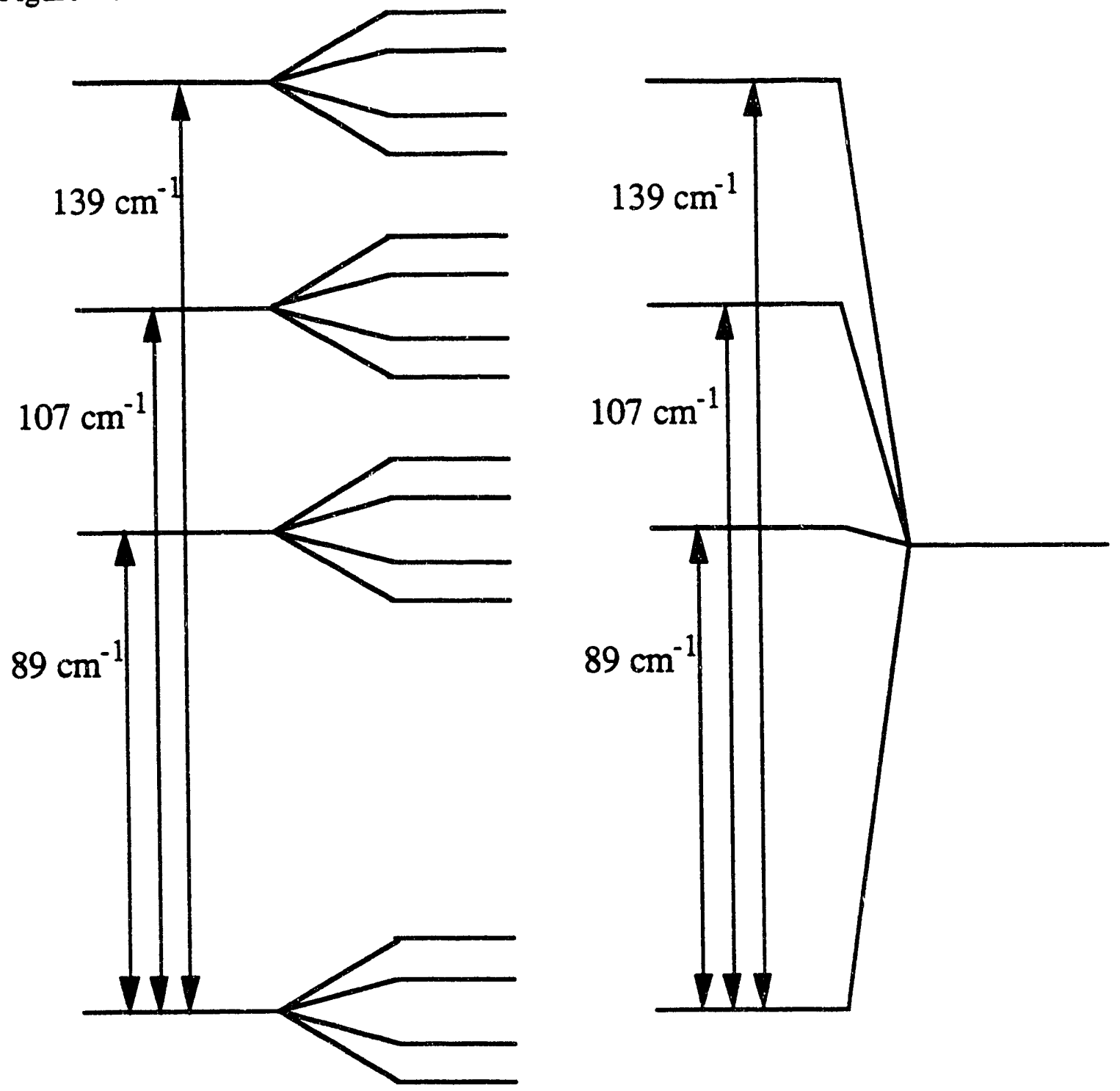

The two possible tunneling scenarios are illustrated for the flipping tunneling splitting. The corrected transition frequencies are shown as double arrows. Each flipping subcomponent is further split by larger barrier tunneling motions ( $\mathrm{cw}-\mathrm{ccw}$, and $\mathrm{C}_{2}$ ) which have been omitted in this Figure. Figure 4.8 describes the entire correlation diagram for a given rovibrational state. In the left hand side, each vibrational state is split due to the flipping tunneling motion. The right hand side, defines the situation for which the flipping tunneling subcomponents correlate directly with the calculated vibrational energies. 
laser is suitable for scanning in this spectral region. This experiment will not only corroborate the general picture outlined in this chapter, it can also provide insight into the two limits of Figure 4.9. Finally, this study is by no means limited to the perdeuterated species. A relatively strong vibration is predicted at $169 \mathrm{~cm}^{-1}$, and this would correspond to a corrected frequency of $152 \mathrm{~cm}^{-1}$ which is conceivably accessible from both the 63 and $70 \mu \mathrm{m} \mathrm{CH} 3 \mathrm{OH}_{3}$ lasers with the extended microwave coverage. Thus, it is clear that this chapter represents only the first step in the study of the water trimer.

Through the detailed study of small water clusters like the trimer, our understanding of the nonpairwise-additive forces operating in hydrogen bonded clusters ${ }^{8}$ and liquids ${ }^{17}$, can ultimately be enhanced dramatically. It is very interesting to note that the harmonic prediction for the ring deformation mode in $\left.\left(D_{2}\right)_{3}\right)_{3}$ at $149 \mathrm{~cm}^{-1}$, is very close to the harmonic approximation for the water dimer stretch near $145 \mathrm{~cm}^{-1}$. The observation of this mode could possibly help in placing a limit on the magnitude of the nonpairwise additive forces.

Possibly the most interesting aspect of the present work is the deduction that the cyclic water trimer is chiral, and that quantum tunneling motions of the equivalent deuterons interconvert the left and right handed enantiomers. This raises interesting possibilities concerning the existence of chiral structures for larger water clusters, such as the stable water clathrates recently studied by Castleman 18,19 . The interaction of such chiral water clusters with another molecule would conceivably break the left/right degeneracy and quench the interconversion tunneling, thereby yielding a complex having a well defined handedness. In this situation, a racemic mixture of chemically equivalent right and left handed forms would result. It is also interesting to consider that the smallest repetitive unit of liquid water exhibits a diamond-like tetrahedral lattice ${ }^{20}$. The asymmetry of the static $\mathrm{H}$-bonding network of liquid water, introduces the possibility of a local chirality existing in the liquid on a time scale shorter than the period of hydrogen tunneling motions or fluctuations which rearrange the H-bonding network. ${ }^{2}$ It seems that no 
previous work has considered either the existence of such transient local chiral structures in liquid water or the possible manifestations of this phenomenon in chemical dynamics. 
1 F. Franks, "Water; A Comprehensive Treatise" (Plenum Press, New York, NY, 1976).

2 F. Sciortino and S. L. Fornili, J. Chem. Phys. 90, 2786 (1989).

3 For examples of such treatments see, R. C. Cohen and R. J. Saykally, Annu. Rev. Phys. Chem. 42, 369 (1991).;J. M. Hutson, Annu. Rev. Phys. Chem. 41, 123 (1990).

4 The references included at the end of Chapter 1 demonstrate the importance of tunneling in systems containing more than two water molecules.

5 N. Pugliano and R. J. Saykally, Science 257, 1937 (1992).

6 J. C. Owicki, L. L. Shipman, and H. A. Scheraga, J. Phys. Chem. 79, 1794 (1975).

7 E. Honeggar and S. Leutwyler, J. Chem. Phys. 88, 2582 (1988).

8 G. Chalasinski, M. M. Szczesniak, P. Cieplak, and S. Scheiner, J. Chem. Phys. 94, 2873 (1991).

9 J. Fowler and H. F. Schaefer, Private Communication.

10 T. R. Dyke, K. M. Mack, and J. S. Muenter, J. Chem. Phys. 66, 498 (1977).

11 Since the axis of rotation for the cis-form is odd, the molecule exhibits chirality - if an nfold axis of rotation exists, for which $\mathrm{n}$ is even, the molecule will not be chiral.

12 K. Balasubramanian and T. R. Dyke, J. Phys. Chem. 88, 4688 (1984).

13 K. L. Busarow, thesis, University of California, Berkeley (1990).

14 The reference of, P. R. Bunker, Molecular Symmetry and Spectroscopy (Academic Press Inc., San Diego, CA, 1979) defines the PI operation. The analysis of this work adopts a method of permuting identical nuclei which is reversed relative to that of Bunker.

15 T. R. Dyke and J. S. Muenter, J. Chem. Phys. 57, 5011 (1972).

16 J. A. Odutola, D. L. Alvis, C. W. Curtis, and T. R. Dyke, Molecular Physics 42, 267 (1981). 
17 H. Saint-Martin, C. Medina-Llanos and I. Ortega-Blake, J. Chem. Phys. 93, 6448 (1990).

18 S. Wei, Z. Shi, and A. W. Castleman, Jr., J. Chem. Phys. 94, 3268 (1991).

19 X. Yang and A. W. Castleman, Jr., J. Am. Chem. Soc. 111, 6845 (1989).

20 D. Eisenberg and W. Kauzmann, The Structure and Properties of Water (Oxford University Press, New York, NY, 1969). 


\section{Chapter 5}

\section{Concluding Remarks}

\subsection{Future Work}

In this work, the versatile capabilities of the FIR laser system have been further demonstrated. The previous two chapters have focussed on the spectral region between 80 $-90 \mathrm{~cm}^{-1}$, in which two reported intermolecular vibrations of small water clusters have been identified and characterized. More precisely, the tunneling dynamics have been thoroughly discussed for both the water dimer and the water trimer van der Waals clusters, and group theoretical models have been used to explain the measured spectral splittings. Since this work represents a new direction of study, it is reasonable to consider future possibilities toward extending the knowledge of intermolecular degrees of freedom in small water clusters. From Tables 1.1 and 4.5 , it is immediately noticed that extremely informative molecular motions are predicted for both the water dimer and the water trimer van der Waals clusters, and these motions should be within the theoretical tuning range of the FIR laser system. An important question to ask is; "Can this method be used to interrogate higher frequency intermolecular vibrations?" When considering the results of Chapters $1-4$, it is believed that the answer is "yes". Extended diode and microwave technology have been tested and utilized with the spectrometer in the present study, and the Ge:Ga photoconductors offer the capability of detection throughout the entire FIR spectral region. However, the difficulty in operating the spectrometer tnward higher frequency is related to the available fixed frequency laser lines from which tunable FIR radiation is produced. Currently continuous spectroscopic coverage exists from ca. $2300-3201 \mathrm{GHz}$ 
from lasers with frequencies greater than $2.4 \mathrm{THz}$, and the existing microwave technology. Furthermore, the $3630 \mathrm{GHz} \mathrm{CH}_{3} \mathrm{OD}$ and $4252 \mathrm{GHz} \mathrm{CH} 3 \mathrm{OH}$ lasers creates window of coverage between 3535 - 3725 and $4157-4347 \mathrm{GHz}$, respectively. This frequency range provides a wonderful opportunity for scanning in a water expansion, however, serious gaps still exist. It is clear that high power, FIR laser development in the higher frequency would benefit this effort. A first step at a possible solution to the lack of powerful lasers might be to redesign the FIR laser cavity with a smaller waveguide diameter, for the present configuration is optimized for frequencies of ca. $1000 \mathrm{GHz}$. A redesign of the laser could permit weak, higher frequency lasers to be detectable with the more sensitive diodes, and this possibility should be discussed as future developments persist.

Within the present scope of the experimental situation, a summary of future scanning for water cluster intermolecular vibrations is presented to conclude this dissertation. Further studies of the water dimer are imminent, and are outlined in this discussion. The 103 and $96 \mu \mathrm{m}$ laser lines should provide the necessary spectral coverage to observe the next $\left(\mathrm{D}_{2} \mathrm{O}\right)_{2}$ intermolecular vibrational band, which has been indirectly identified in Chapter 3 through its perturbing influence on the $82.6 \mathrm{~cm}^{-1}$ acceptor bending coordinate. This vibration is presumably the $v_{8}$ donor torsion which is estimated to lie in the $90-105 \mathrm{~cm}^{-1}$ spectral range for the $\mathrm{D}_{2} \mathrm{O}$ dimer. Although Reference 20 of Chapter 1 predicts that this vibration should be approximately five-fold weaker than the acceptor bend, it should still be observable with the present level of sensitivity. Furthermore, when realizing that the value of the A rotational constant is $122 \mathrm{GHz}$, and large tunneling splittings will almost certainly be present, it becomes clear that VRT bands between various $\mathrm{K}_{\mathrm{a}}$ manifolds will generate spectra over a large frequency range. Thus, these two lasers will be critical in identifying this vibration the acceptor bending coordinate in the normal isotopomer of the water dimer. Finally, the water dimer stretching frequencies of 145 and $151 \mathrm{~cm}^{-1}$ for the perdeuterated and normal isotopomers, respectively, should be detected near the $70 \mu \mathrm{m}$ laser line by using the UPC detector. This prediction is rather reliable, 
compared to the other intermolecular vibrational bands, so searching in a water expansion at this frequency would be informative.

Scanning should also be accomplished in an $\mathrm{H}_{2} \mathrm{O} / \mathrm{D}_{2} \mathrm{O}$ mixture to identify the mixed isotopomers of the water dimer. Reference 32 of Chapter 3 explains the group theoretical manifestations of the tunneling motions for all of the possible water dimer isotopomers. From this the VRT tunneling splittings may be identified as was partially done for the $\mathrm{D}_{2} \mathrm{O}$ - DOH species. By characterizing each of the isotopomers, the individual tunneling motions may be examined through the characterization of the resulting VRT levels. These experiments will permit for an experimental determination of the influence of each tunneling motion on the intermolecular vibrational coordinates. Clearly the $\mathrm{D}_{2} \mathrm{O}-\mathrm{DOH}$ isotopomer should be targeted first, for two of the four possible VRT bands originating from $K_{a}=0$ have already been located.

Also in this spectral region lie the predicted values for the remaining flipping intermolecular vibrational motions in the perdeuterated water trimer van der Waals cluster. Since the barriers for the $\mathrm{H}$-bond breaking tunneling motions are so much larger than the barrier for the flipping tunneling motion, the F state multiplet signature may be present throughout the FIR spectral region, because the high barrier motions may not adequately couple to the vibration, as in the case of the water dimer. Thus, it would be wise to continue this effort with hopes of observing more of the same kind of quartet features.

At the present time, it is unclear which of the tunneling limits presented in Figure 4.9 , apply to the cluster, and the observation of the next vibrational band, predicted to be near $107 \mathrm{~cm}^{-1}$, will further develop the present understanding of the water trimer. Of course, such an observation must obey the rigorous selection rules presented in Chapter 4 , as $\Gamma_{\mathrm{i}^{+}}{ }^{-}<->\Gamma_{\mathrm{i}^{-}}$. This selection rule makes the situation of the right handed side of Figure 4.9 extremely unfavorable for observing spectra off of the $96 \mu \mathrm{m}$ laser. If the flipping tunneling motion correlates directly to intermolecular vibrational modes, as shown in this portion of the figure, then each of the "+" and "-" parity labels corresponding to the states 
in the correlation diagram of Figure 4.8, should be assigned to the $\checkmark$,brational states. For example, if this limit exists, the ground state will presumably possess a parity label of "+", thus forcing the observed VRT states located near $89 \mathrm{~cm}^{-1}$ to correspond to "-" parity labels. If the predicted vibration also has a "." parity label associated with its VRT components, then the transition should be observable. On the contrary, if the tunneling limit on the left hand side of Figure 4.9 is appropriate, each vibrational state will split into four sublevels due to the flipping tunneling motion, upon which each of these will be further split due to the remaining tunneling coordinates, and transitions near $107 \mathrm{~cm}^{-1}$ will almost certainly be present. Thus, by searching for other $F$ states near the observed vibration, and by scanning toward higher frequency, the correct tunneling limit can defined.

Throughout the course of scanning, many unassigned transitions have been detected and categorized, of which many of them almost certainly belong to larger water clusters. As ab initio predictions for the tetramer and larger clusters become available, the power of the FIR laser spectrometer may be employed toward the study of these intriguing molecular species. 

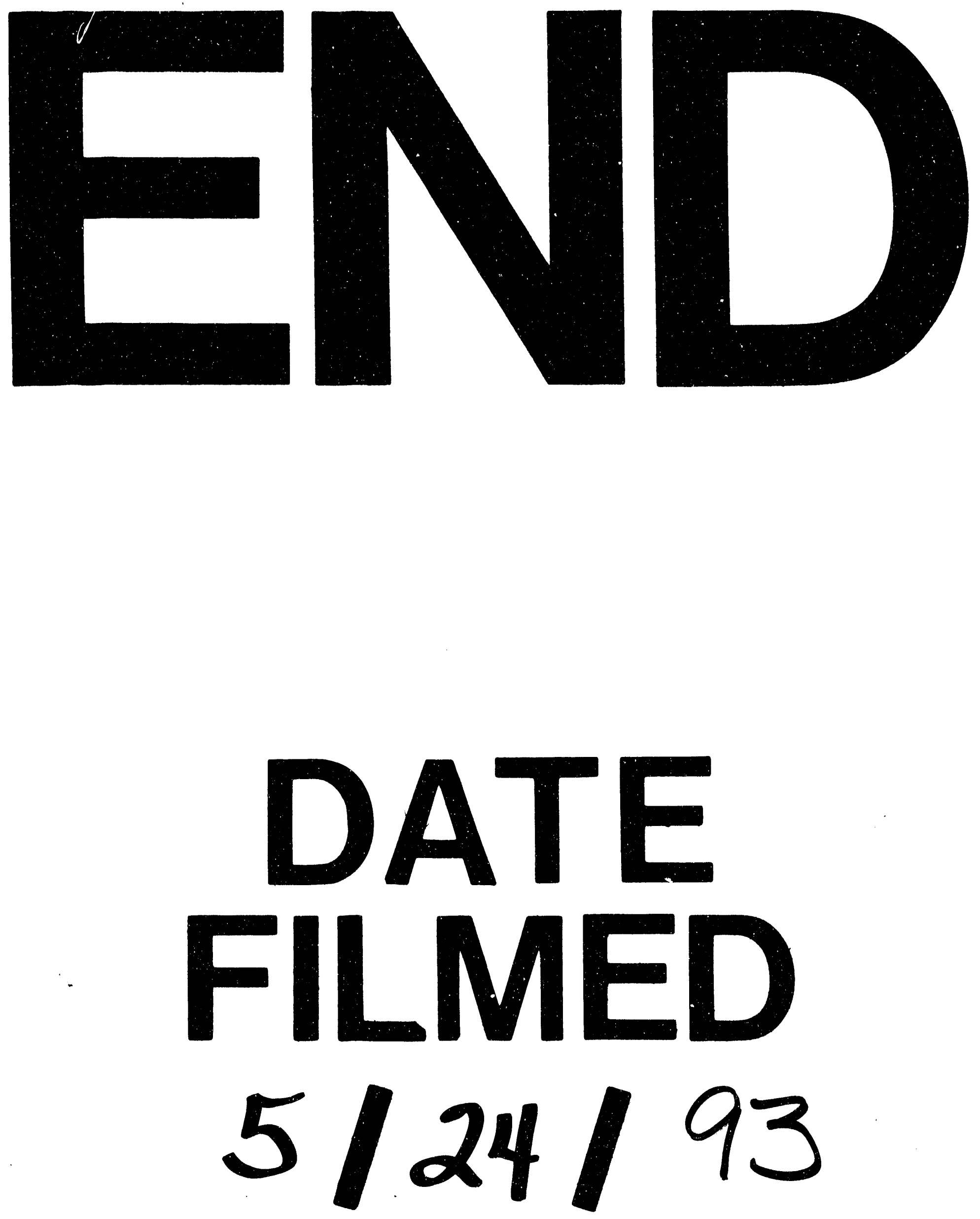

1 
